\title{
"Non-Essential" Proteins of HSV-1 with Essential Roles In Vivo: A Comprehensive Review
}

\author{
Christos Dogrammatzis ${ }^{+}(\mathbb{D})$, Hope Waisner ${ }^{+} \mathbb{D}$ and Maria Kalamvoki * \\ Department of Microbiology, Molecular Genetics, and Immunology, University of Kansas Medical Center, \\ Kansas City, KS 66160, USA; cdogrammatzis@kumc.edu (C.D.); h721w416@kumc.edu (H.W.) \\ * Correspondence: mkalamvoki@kumc.edu \\ † These authors contributed equally to this work.
}

Citation: Dogrammatzis, C.; Waisner, H.; Kalamvoki, M. “Non-Essential” Proteins of HSV-1 with Essential Roles In Vivo: A Comprehensive Review. Viruses 2021,13,17. https:// dx.doi.org/10.3390/v13010017

Academic Editor: Oliver Schildgen Received: 24 November 2020 Accepted: 18 December 2020 Published: 23 December 2020

Publisher's Note: MDPI stays neutral with regard to jurisdictional claims in published maps and institutional affiliations.

Copyright: () 2020 by the authors. Licensee MDPI, Basel, Switzerland. This article is an open access article distributed under the terms and conditions of the Creative Commons Attribution (CC BY) license (https: / / creativecommons.org/ licenses/by/4.0/).

\begin{abstract}
Viruses encode for structural proteins that participate in virion formation and include capsid and envelope proteins. In addition, viruses encode for an array of non-structural accessory proteins important for replication, spread, and immune evasion in the host and are often linked to virus pathogenesis. Most virus accessory proteins are non-essential for growth in cell culture because of the simplicity of the infection barriers or because they have roles only during a state of the infection that does not exist in cell cultures (i.e., tissue-specific functions), or finally because host factors in cell culture can complement their absence. For these reasons, the study of most nonessential viral factors is more complex and requires development of suitable cell culture systems and in vivo models. Approximately half of the proteins encoded by the herpes simplex virus 1 (HSV-1) genome have been classified as non-essential. These proteins have essential roles in vivo in counteracting antiviral responses, facilitating the spread of the virus from the sites of initial infection to the peripheral nervous system, where it establishes lifelong reservoirs, virus pathogenesis, and other regulatory roles during infection. Understanding the functions of the non-essential proteins of herpesviruses is important to understand mechanisms of viral pathogenesis but also to harness properties of these viruses for therapeutic purposes. Here, we have provided a comprehensive summary of the functions of HSV-1 non-essential proteins.
\end{abstract}

Keywords: HSV-1 non-essential proteins; HSV-1 egress; HSV-1 envelopment; innate immunity; HSV-1 based therapies; gene silencing

\section{Introduction}

The large family of DNA viruses, Herpesviridae, have co-evolved with mammals for millions of years [1,2]. The family Herpesviridae is further divided into three subfamilies, Alphaherpesvirinae, Betaherpesvirinae, and Gammaherpesvirinae. Herpes simplex virus type 1 (HSV-1), a member of Alphaherpesvirinae, is one of the most well-studied representatives of this family of viruses and will be the focus of this review.

HSV-1 is an enveloped dsDNA virus, which has a genome size of about $152 \mathrm{~kb}$ and virion size of about 150-300 nm in diameter [3]. The virion contains the envelope decorated with viral glycoproteins and a proteinaceous layer known as the tegument, which surrounds the capsid of the virus containing the genome. HSV-1 is an important human pathogen, with approximately $80 \%$ of the human population infected [3]. Symptoms of HSV-1 infection vary, from lesions in the oral-facial region ("cold sores"), to herpes keratitis, the leading cause of infectious blindness, to herpes encephalitis, which can be fatal. When HSV-1 encounters a host, it will first infect the mucosal epithelial cells in the oral-facial region (although HSV-1 also causes genital infections). It is in these cells that the virus undergoes lytic replication. The virus will then infect innervating sensory neurons, travel anterograde to the trigeminal ganglia (TG), and establish latency, where it will remain for the life of the infected individual. When HSV-1 undergoes latency there are very few genes expressed, including an 8.3-kb region known as the latency-associated transcript 
(LAT), which is a long non-coding regulatory RNA spliced to about 1.5- and 2-kb introns that have regulatory roles on viral genes expression, and a 6.3-kb exon encoding multiple microRNAs, which target many of the IE genes and other lytic genes, thus suppressing viral replication [4-10]. It seems that LAT may be important for reactivation of HSV-1 from latency and for blocking apoptosis [11-18]. Periodically, HSV-1 will reactivate from latency due to stress, immunosuppression, or other stimuli, and newly produced virions will travel retrograde to the initial site of infection. There is currently no cure for HSV-1 and no vaccine.

There are three classes of viral genes for HSV-1 and they are expressed in a cascade fashion $[19,20]$. The virus first encodes the immediate-early (IE) or alpha $(\alpha)$ genes (ICP0, ICP4, ICP27, ICP22, or ICP47) whose products are important for expression of the next class of viral genes, the early $(E)$ or beta $(\beta)$ genes. The early genes encode proteins largely involved in viral DNA replication and, along with the immediate-early genes, facilitate the expression of the late class of viral genes. The late $(\mathrm{L})$ or gamma $(\gamma)$ class of viral genes express proteins involved in virion assembly and egress. HSV-1 genes are also divided based on stretches of unique sequences in the genome. Therefore, there are a class of unique long $\left(\mathrm{U}_{\mathrm{L}}\right)$ and unique short $\left(\mathrm{U}_{\mathrm{S}}\right)$ genes depending on which region of the genome the gene is expressed from. These unique regions are flanked by inverted repeats. Thus, the HSV-1 genome is structured as follows: $T R_{L}-U_{L}-I R_{L}-I R_{S}-U_{S}-T R_{S}$. There are about $58 U_{L}$ genes and about $13 \mathrm{U}_{\mathrm{S}}$ genes that have been characterized for functionality, though there are more viral genes that have not been well characterized or described (Dolan 1998).

Interestingly, although HSV-1 is known to encode 80 genes, it has also been found that about half of these genes are non-essential for viral replication in cell culture [21,22]. Essential genes of HSV-1 are involved in viral DNA replication, the transcription of certain viral genes, genes encoding capsid proteins, genes encoding viral DNA packaging proteins, and some envelope glycoproteins. HSV-1 genes determined to be non-essential are involved in nucleic acid metabolism, combating various host responses to infection, facilitating optimal viral replication, facilitating primary envelopment, virus pathogenesis, or other functions that are not yet characterized (Table 1). While deletion of the non-essential genes in cell culture does not inhibit viral replication, these genes are generally essential for replication in the natural human host as mutant viruses deleted of non-essential genes have rarely been isolated from a patient. One example are mutants in the viral glycoprotein $\mathrm{gC}$ that have been recovered from patients with recurrent herpes keratitis $[23,24]$.

Table 1. Non-essential genes of HSV-1, corresponding proteins, their location on the HSV-1 virion, and their function. Pink: tegument proteins, blue: accessory proteins, yellow: envelope proteins, green: capsid proteins.

\begin{tabular}{|c|c|c|c|}
\hline Gene & Protein & Location on Virion & $\begin{array}{l}\text { Function } \\
\end{array}$ \\
\hline RL1 or $\gamma 134.5$ & ICP34.5 & tegument & $\begin{array}{l}\text { Prevents host translational shutoff and } \\
\text { autophagy }\end{array}$ \\
\hline$R L 2$ or $\alpha 0$ & ICP0 & tegument & $\begin{array}{c}\text { Promiscuous transactivator of genes, } \\
\text { disrupts repressor complexes, E3 ubiquitin } \\
\text { ligase, inhibits innate immunity, modulates } \\
\text { endocytosis, etc. }\end{array}$ \\
\hline UL2 & uracil-DNA glycosylase & accessory & nucleic acid metabolism \\
\hline UL3 & & accessory & \\
\hline UL4 & & accessory & \\
\hline UL7 & & tegument & Virion assembly and egress \\
\hline UL10 & gM & envelope & Host and viral protein trafficking \\
\hline UL11 & & tegument & Cytoplasmic envelopment \\
\hline UL12 & & accessory & Nucleic acid metabolism \\
\hline UL12.5 & & accessory & Involved in depleting mtDNA \\
\hline UL13 & Ser/thr protein kinase & tegument & $\begin{array}{l}\text { Blocking innate immune responses, } \\
\text { supporting viral protein synthesis }\end{array}$ \\
\hline
\end{tabular}


Table 1. Cont.

\begin{tabular}{|c|c|c|c|}
\hline Gene & Protein & Location on Virion & Function \\
\hline UL16 & & tegument & Cytoplasmic envelopment \\
\hline UL20 & & envelope & Glycoprotein trafficking \\
\hline UL21 & & tegument & Promotes capsid egress to the cytoplasm \\
\hline UL23 & thymidine kinase (TK) & tegument & Broad spectrum nucleoside kinase \\
\hline UL24 & & accessory & Glycoprotein trafficking, nucleolus dispersal \\
\hline UL31 & & accessory & $\begin{array}{c}\text { Component of the nuclear egress complex } \\
\text { (NEC), promotes primary nuclear } \\
\text { envelopment }\end{array}$ \\
\hline UL34 & & accessory & $\begin{array}{c}\text { Component of the nuclear egress complex } \\
\text { (NEC), promotes primary nuclear } \\
\text { envelopment }\end{array}$ \\
\hline UL35 & VP26 & capsid & $\begin{array}{l}\text { Affects DNA packaging, mediates capsid } \\
\text { assembly, trafficking post viral entry }\end{array}$ \\
\hline UL39 & $\begin{array}{l}\text { RR1 (ribonucleotide } \\
\text { reductase) }\end{array}$ & accessory & $\begin{array}{l}\text { Part of the ribonucleotide reductase (RR) } \\
\text { complex, converts ribonucleotide } \\
\text { diphosphates to corresponding } \\
\text { deoxyribonucleotides, allowing for virus } \\
\text { replication particularly in non-dividing cells }\end{array}$ \\
\hline UL40 & $\begin{array}{l}\text { RR2 (ribonucleotide } \\
\text { reductase) }\end{array}$ & accessory & $\begin{array}{l}\text { Part of the ribonucleotide reductase (RR) } \\
\text { complex, converts ribonucleotide } \\
\text { diphosphates to corresponding } \\
\text { deoxyribonucleotides, allowing for virus } \\
\text { replication particularly in non-dividing cells }\end{array}$ \\
\hline UL41 & VHS & tegument & $\begin{array}{l}\text { Viral RNase, degrades host transcripts and } \\
\text { blocks antiviral responses }\end{array}$ \\
\hline$L 43$ & & tegument & \\
\hline UL44 & $\mathrm{gC}$ & envelope & $\begin{array}{l}\text { Mediates viral binding to heparan sulfate, } \\
\text { regulates entry by a low-pH pathway }\end{array}$ \\
\hline UL45 & & envelope & $\begin{array}{l}\text { Required for syncytia formation during } \\
\text { HSV-1 gB syn infection }\end{array}$ \\
\hline UL46 & VP11/12 & tegument & $\begin{array}{l}\text { Regulation of transcription, activates } \\
\text { pathways for cell survival, blocks pathways } \\
\text { for innate immunity activation }\end{array}$ \\
\hline UL47 & VP13/14 & tegument & $\begin{array}{l}\text { Regulation of transcription, modulating } \\
\text { post-transcriptional processing of mRNAs }\end{array}$ \\
\hline UL49 & VP22 & tegument & $\begin{array}{l}\text { Facilitates viral gene expression, protein } \\
\text { expression, and DNA replication; inhibits } \\
\text { inflammasome }\end{array}$ \\
\hline UL49.5 & $\mathrm{gN}$ & envelope & Binding partner of gM \\
\hline UL50 & & tegument & Nucleic acid metabolism \\
\hline UL51 & & tegument & $\begin{array}{c}\text { Participates in cytoplasmic envelopment; } \\
\text { facilitates virus spread from cell-to-cell; } \\
\text { recruits UL7 to tegument }\end{array}$ \\
\hline UL53 & gK & envelope & $\begin{array}{l}\text { Participates in virion egress from host cell; } \\
\text { regulates virus entry and fusogenic activity } \\
\text { of the virion; complexes with UL20 }\end{array}$ \\
\hline UL55 & & tegument & Participates in cytoplasmic envelopment \\
\hline UL56 & & tegument & Participates in cytoplasmic envelopment \\
\hline US1 & ICP22 & accessory & $\begin{array}{l}\text { Regulates viral late gene expression; } \\
\text { facilitates formation of complexes important } \\
\text { for protein folding; participates in primary } \\
\text { envelopment; blocks immune responses }\end{array}$ \\
\hline US1.5 & & accessory & Participates in viral gene transcription \\
\hline US2 & & tegument & Protein trafficking \\
\hline US3 & Ser/thr protein kinase & tegument & $\begin{array}{l}\text { Blocks apoptosis, enhances viral gene } \\
\text { expression, facilitates capsid nuclear egress, } \\
\text { phosphorylates numerous substrates }\end{array}$ \\
\hline
\end{tabular}


Table 1. Cont.

\begin{tabular}{|c|c|c|c|}
\hline Gene & Protein & Location on Virion & Function \\
\hline US3.5 & Ser/thr protein kinase & tegument & $\begin{array}{c}\text { Phosphorylates substrates but cannot block } \\
\text { apoptosis and does not facilitate nuclear } \\
\text { egress }\end{array}$ \\
\hline US4 & gG & envelope & Regulation of chemokines \\
\hline US5 & gJ & envelope & Inhibits apoptosis and cell stress pathways \\
\hline US7 & gI & envelope & $\begin{array}{l}\text { Enhances virus spread from cell-to-cell; } \\
\text { facilitates anterograde transport of virions } \\
\text { after reactivation from latency; important for } \\
\text { neurovirulence }\end{array}$ \\
\hline US8 & gE & envelope & $\begin{array}{l}\text { Enhances virus spread from cell-to-cell; } \\
\text { facilitates anterograde transport of virions } \\
\text { after reactivation from latency; important for } \\
\text { neurovirulence }\end{array}$ \\
\hline US8.5 & & accessory & Localizes in the nucleoli \\
\hline US9 & & tegument & $\begin{array}{l}\text { Enhances virus spread from cell-to-cell; } \\
\text { facilitates anterograde transport of virions } \\
\text { after reactivation from latency; important for } \\
\text { neurovirulence }\end{array}$ \\
\hline US10 & & tegument & Important for neurovirulence \\
\hline US11 & & tegument & $\begin{array}{c}\text { Block PKR activation and shutoff of host } \\
\text { translation; block IFN induction; regulation } \\
\text { of virus genes expression; trafficking of } \\
\text { unenveloped capsids }\end{array}$ \\
\hline US12 & ICP47 & accessory & $\begin{array}{l}\text { Prevents MHC I antigen presentation, } \\
\text { supports neurovirulence }\end{array}$ \\
\hline
\end{tabular}

It is of great interest to understand the roles of non-essential genes to better understand virus-host interactions. Moreover, the non-essential genes have properties that make them attractive for the development of therapeutics. There are varying degrees of deficiency of viruses mutated for non-essential genes when grown in cell culture, and for some of these genes, the defect is cell type specific $[25,26]$. There is still much to learn about the non-essential genes of HSV-1. Here, we present a comprehensive analysis of the current understanding of the roles of non-essential genes of HSV-1. We explore the functions ascribed to these genes and their corresponding proteins, the potential treatment and therapeutic avenues that can be explored based on the functions and characterization of select HSV-1 non-essential genes, and the complex and intricate roles of non-essential genes in HSV-1 infection.

\section{Repressors of Gene Silencing, Viral Transactivators, and Host Evasion Factors}

\section{1. $R L 2$ or $\alpha 0$ (ICP0)}

The infected cell protein 0 (ICP0) of HSV-1 was first reported as a nuclear phosphoprotein with an essential role in cell cultures only at low multiplicity of infection (MOI). ICP0 was deemed to be non-essential at high multiplicities of infection in cell cultures, but viral gene expression was reduced $[19,27-33]$. In certain cell lines, particularly cancer cell lines, such as the human osteosarcoma (U2OS), an ICP0-null virus replicates as efficiently as wild-type virus, which may be due to impaired recruitment of antiviral factors to the sites of viral gene transcription and DNA replication and/or due to lack of certain restriction factors $[26,28,30,32,34,35]$. Genes coding for ICP0 are present in the genomes of simplex and varicelloviruses, but they are absent from the mardivirus genus. These proteins show strong sequence homology to ICP0 within the RING (Really Interesting New Gene) finger domain. Orthologs of ICP0 are also present in lymphocryptoviruses (e.g., EBV) and the cytomegalovirus (CMV) [36-38]. The functions of ICP0 are broad, from activation of transcription and chromatin remodeling, to evasion of antiviral responses, cell cycle effects, interfering with DNA damage responses, and endocytosis. 
In early studies, ICP0 was found with ICP4 to stimulate ICP8 expression in transfection assays [39,40]. Furthermore, it was shown to function as a potent transactivator of different genes introduced into cells by transfection or infection, including the viral thymidine kinase (TK) gene and ICP6 gene, the human immunodeficiency virus (HIV) LTR, and several human papillomavirus (HPV) genes [41-50]. In fact, ICP0 was found to stimulate the expression of all three classes of HSV genes [31,51]. Therefore, ICP0 was proposed to be a promiscuous transactivator of gene expression.

ICP0 also functions as an E3 ubiquitin ligase and most substrates ubiquitinated by ICP0 appear to be targeted for degradation (Figure 1A). This activity of ICP0 was mapped to residues 116-156, where there is a $\mathrm{Zn}^{2+}$-binding RING finger domain [52-55]. To exert its E3 ubiquitin ligase function, ICP0 forms a complex with different ubiquitin conjugation enzymes, including $\mathrm{UbcH} 5 \mathrm{a}$ and $\mathrm{UbcH} 6$ [52,56-60]. Major targets of ICP0 are components of the nuclear domain 10 (ND10) bodies. As a DNA virus, the genome of HSV-1 transcribes and replicates in the nucleus. The host attempts to block viral gene expression and replication by entrapping the viral DNA in promyelocytic leukemia (PML)-nuclear bodies (NBs) and depositing histones and other repressor complexes on it. The main protein that orchestrates the formation of ND10 bodies is the PML. Other components of ND10s include the Sp100, Daxx, Mre 11, ATM, ATRX, p53, and others. ICP0 disrupts the ND10s by causing degradation of the different isoforms of PML, Sp100, and potentially of other proteins (Figure 1A) [34,59,61-77] Notably, several components of the ND10 bodies are interferon inducible genes, which underscores the synergy between gene silencing mechanisms and innate immunity in suppressing HSV-1 gene expression. ICP0-null viruses or E3 ubiquitin ligase mutants have viral DNA entrapped in PML-NBs at low MOI and display reduced transactivation activity and ability to block antiviral responses [34,64,78-83]. ICP0 E3 ligase-deficient viruses are hypersensitive to interferon, replicate poorly, and fail to reactivate efficiently from neuronal latency [25,55,67-71]. Based, on these observations, Dr. Kalamvoki's group recently developed a high-throughput assay to screen for ICP0-E3 ubiquitin ligases inhibitors [72]. This assay is proximity based and takes advantage of the fact that ICP0 is autoubiquitinated and degraded during infection and that this ICP0 autoubiquitination can occur in vitro using the purified protein encoded by the exon II of ICP0 (contains the RF domain), UbcH5a, and $\mathrm{Ub}[60,73,74]$. Screening a small compound library, Dr. Kalamvoki's group identified potential scaffolds that can interfere with the ICP0 E3 ubiquitin ligase activity [72].

ICP0 has seven SUMO-interacting motif (SIM)-like sequences (SLSs), and multiple ND10 components, including PML and SP100, are SUMOylated; therefore, ICP0 could bind to them (Figure 1A) [34,56,75-81]. It has been found that inhibition of cellular ubiquitination led to an increase of SUMOylated proteins that ended up accumulating at PML-NBs [82]. ICP0 utilizes both SUMO-dependent and SUMO-independent mechanisms to degrade Sp100 and multiple PML isoforms in an effort to prevent restriction of the virus by the host $[34,56,75,76,78,79,83,84]$. Other proteins could also be the target of SUMO-dependent degradation by ICP0 $[75,77,81]$. Specifically, SUMO-dependent degradation of MORC3 by ICP0, which associates with Sp100, has been observed and this occurs in a RINGfinger-dependent manner and appears to diminish the association of PML-NBs with viral DNA [85]. Additionally, there has been a function ascribed to ICP0 SUMO-SIM interactions at the ND10s to modulate the DNA damage response (DDR) during infection [86,87]. For example, the DNA repair function of the DNA-dependent protein kinase (DNA-PK) is inhibited by ICP0 through degradation of its catalytic subunit and this facilitates virus replication [88-90]. Additionally, ICP0 mediates the degradation of two E3 ubiquitin ligases RFN8 and RFN168 that act as mediators of the ATM pathway and trigger recruitment of downstream effectors to sites of double-strand DNA breaks [91-95]. More work will need to be done to characterize the degradation of SUMOylated proteins by ICP0 that are not related to the ND10s. The ability for ICP0 to interrupt SUMO interactions and to degrade SUMOylated proteins during infection is likely a strategy to modify the cellular proteome to both prevent antiviral responses and promote the infection $[34,76,83]$. 
In tandem with the dispersion of ND10 bodies, ICP0 activates the viral chromatin (Figure 1B). Immediately after its release in the nucleus, HSV-1 DNA associates with repressive histones and other repressor complexes [96-98]. However, markers of active gene expression label the viral chromatin during lytic infection, such as tri-methylation of histone $\mathrm{H} 3$ at lysine 4 (H3K4) and acetylation of $\mathrm{H} 3$ at lysine 9 and lysine 14 [97-99], while suppressive epigenetic modifications of histone H3 (H3K9me3 and H3K27me3) are removed in an ICP0-dependent manner (Figure 1B) $[65,100,101]$. ICP0 was also found to associate with class II HDACs in vitro and control their repressor activity $[102,103]$. In addition, ICP0 seems to promote histone acetylation, as demonstrated using inhibitors of histone deacetylases [103-105]. This is also supported by the fact that ICP0 recruits to the viral genome the histone acetyltransferase CLOCK through interaction with the circadian regulator protein BMAL1. This leads to recruitment of additional viral transactivators ICP4, ICP22, ICP27, and part of the host transcription complex TFIID [106-108]. Tandemly, ICP0 disrupts repressor complexes, such as the REST/CoREST/HDAC complex and LSD1 [109-112]. ICP0 disperses the REST/CoREST/HDAC1/2/LSD1 through interaction with CoREST in an effort to promote HSV-1 gene expression and DNA replication (Figure 1B) [78,112-115]. It was also found that the interferon-inducible gene 16 (IFI16), Daxx, and ATRX proteins serve to restrict the virus, likely through sensing of viral DNA and obstructing replication and causing deposition of silencing histone $\mathrm{H} 3[34,65,100,116-120]$. ICP0 induces the degradation of ATRX and IFI16 [121-125]. Degradation of ATRX seems to be secondary to PML degradation, while depletion of ICP0 appears to be both ICP0 dependent and independent.

ICP0 has also been shown to harness cell cycle components to support the infection. Thus, ICP0 was found to recruit cyclin D3 and the kinase cdk4 to ND10s to enable viral gene transcription and DNA replication, which was also supported by the fact that ICP0 nuclear-to-cytoplasmic translocation was enabled by cyclin D3 (Figure 1B) $[108,126,127]$. ICP0 has been found to arrest cells in the G2/M phase to promote virus replication by activating the checkpoint kinase 2 (Chk2) [128]. Consistent with these roles of ICP0, it was also found to degrade the centromere proteins CENP-A, CENP-B, and CENP-C, inducing the interphase centromere damage response (iCDR) (Figure 1A) [129-132]. In addition to this disturbance to the cell cycle, it has been found that ICP0 degrades the DNA-interacting protein TPP1, leading to transcription of telomere repeat-containing RNA activation (TERRA) and increased viral replication [133].

As mentioned earlier, there are interwoven relationships between gene silencing and innate immunity and it is not coincidence the ICP0 targets them both. ICP0-null and other ICP0 mutant viruses displayed increased sensitivity to interferon both in vivo and in vitro $[32,70,134,135]$. As discussed above, ICP0 blocks the nuclear pattern recognition receptors (PRRs) IFI16 and DNA-PKs, which may also impact the cGAS and STING DNA sensing pathway $[119,122-124,136,137]$. Inhibition of STING-dependent immune responses involves ICP0 as ICP0-null virus growth is partially rescued in cells with impaired STING signaling $[25,26]$. Furthermore, ICP0 was found to reduce the levels of the Toll-like receptor 2 (TLR2) adaptors MyD88 (myeloid differentiation factor 88) and the Mal (MyD88 adaptorlike protein) TIRAP (TIR domain-containing adaptor protein), thus blocking immune responses through this pathway (Figure 2A) [138]. Overall, ICP0 has been proposed to inhibit IRF3 and IRF7-dependent immune responses to sequester these proteins away from host chromatin [139-141]. ICP0 was also recently found to have a role in autophagy inhibition through causing the downregulation of p62/SQSTM1 and OPTN autophagy adaptor proteins in a proteasome-dependent and RING finger-independent mechanism (Figure 2C) [142]. It was also demonstrated that the cytoplasmic ICP0 is most likely involved in this function. Another target of ICPO is the deubiquitylating enzyme USP7 (ubiquitin-specific protease 7) or HAUSP. USP7 appears to bind and stabilize ICP0, but ICP0 degrades USP7 late during infection in a RING finger-dependent manner $[58,78,79,143,144]$. One reason why the virus could promote degradation of USP7 is because it has a major role in TLR- and TNFa receptor (TNFR)-induced gene expression [145]. 
Most functions of ICP0 discussed above are performed while in the nucleus. However, ICP0 translocates to the cytoplasm after enabling viral gene expression, where it remains for the reminder of the infection. The cytoplasmic functions of ICP0 remain unexplored. Dr. Roizman's group first described an interaction of ICP0 with the endocytosis adaptor CIN85 [146]. Dr. Kalamvoki's group has built upon these findings and reported that ICP0 promotes endocytosis of the viral entry receptor Nectin-1 (Figure 2A) [147]. This is perhaps a mechanism that ensures spread of progeny viruses to uninfected cells. CIN85 forms a complex with the $\mathrm{Cbl} \mathrm{E3} \mathrm{ligase} \mathrm{that} \mathrm{is} \mathrm{involved} \mathrm{in} \mathrm{endocytosis} \mathrm{of} \mathrm{multiple} \mathrm{surface}$ components. Thus, ICP0 through CIN85 and Cbl could modulate the surface of infected cells to suppress antiviral responses.

Finally, the role of ICPO has also been investigated during the latent stage of the virus. ICP0 appears to be important for efficient virus reactivation from latently infected trigeminal ganglia (TG) in mouse ocular infections [67,68,71,148-151]. ICP0 is also required for VP16-dependent viral reactivation $[67,152]$. While ICP0 is important for balancing lytic and latent infection, it is still not fully understood what its specific role is in this process.

A.

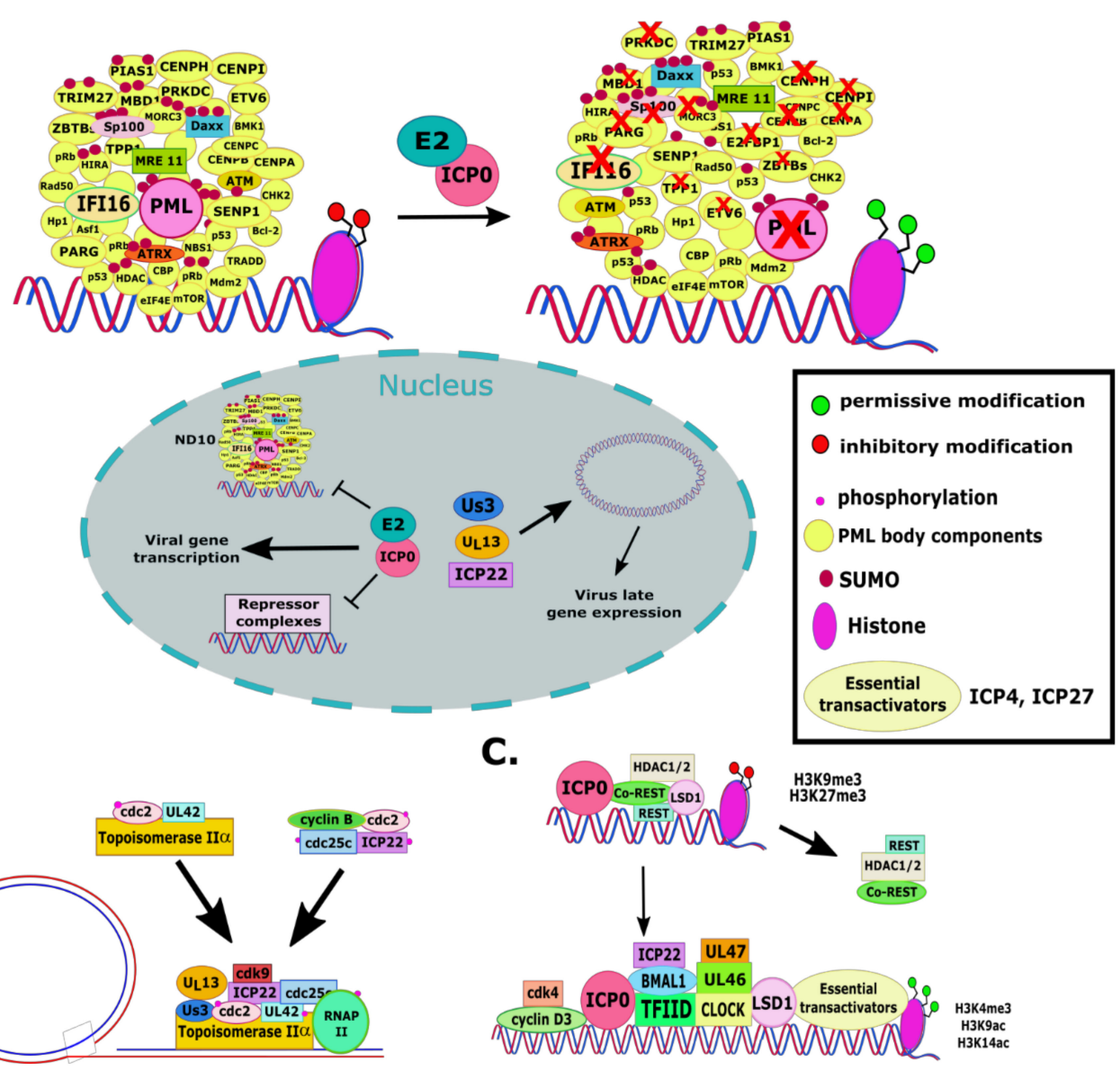

Figure 1. Nuclear functions of non-essential HSV-1 proteins. HSV-1 encodes multiple proteins able to counteract antiviral host responses within the nucleus. (A): ICP0 functions as an E3 ligase ubiquitin ligase that degrades ND10 components that encapsulate the viral genome in the nucleus, including PML, Daxx, SP100, centromeric proteins, and others. The degradation of IFI16 involves multiple factors. These events facilitate initiation of viral gene transcription. (B): The viral protein ICP0 is also known to disrupt repressor complexes that silence the viral genome, as well as recruit factors to enable viral gene transcription. Altogether, ICP0 facilitates permissive histone modifications, while suppressing silencing modifications, to enable for viral gene expression. (C): The viral kinases $U_{S} 3$ and $U_{L} 13$, with ICP22, are known to facilitate viral late gene expression, which occurs through the recruitment of host factors, such as Topoisomerase II $\alpha$ and RNA polymerase II, to the sites of DNA replication in the nucleus. Together, these non-essential viral proteins are important for optimal expression of other viral genes and for viral DNA replication. 


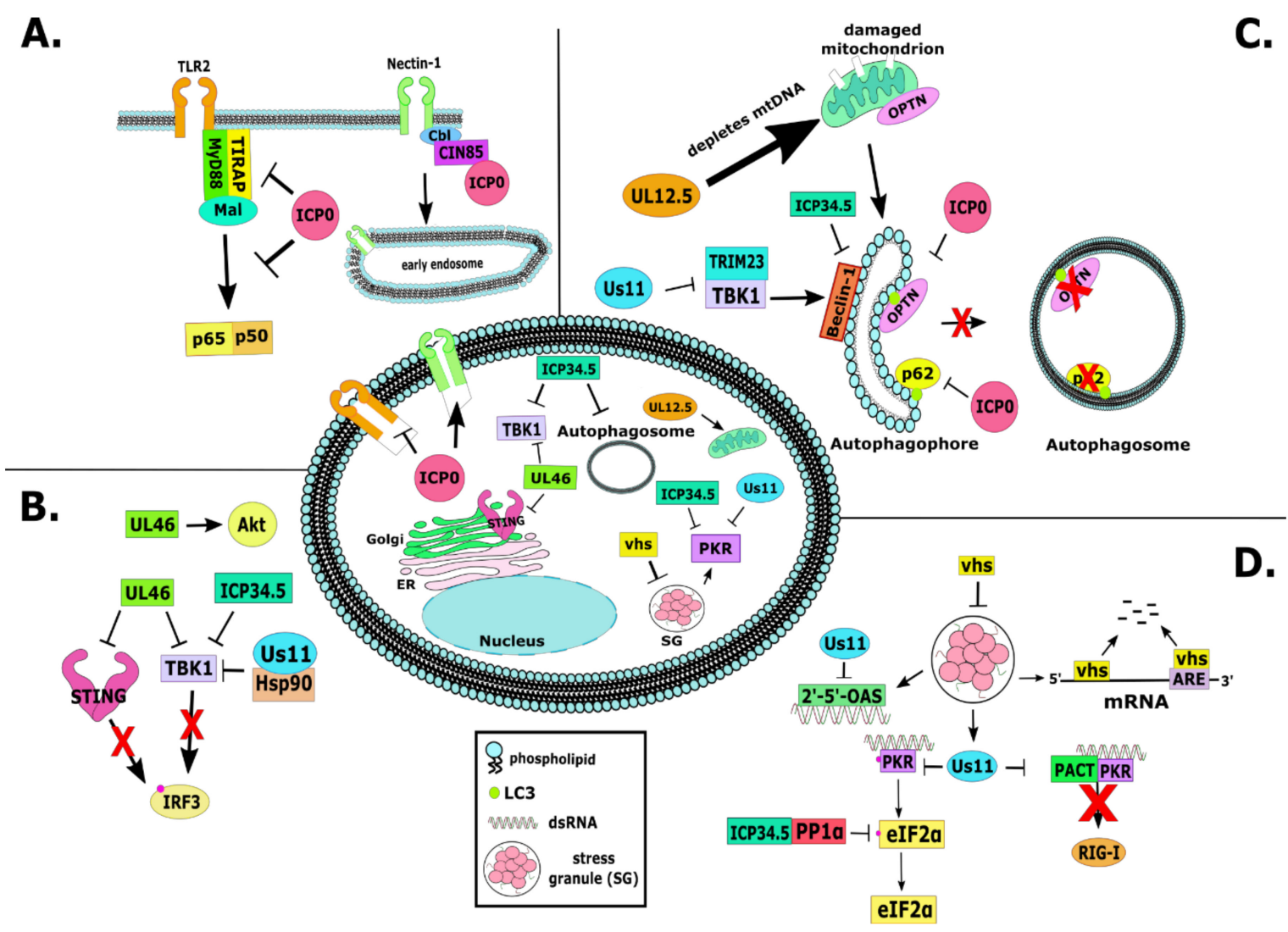

Figure 2. Cytoplasmic functions of some non-essential HSV-1 proteins. (A): ICP0 participates in two major functions in the cytoplasm. First, ICP0 degrades the TLR2 adaptors TIRAP and Mal, thus blocking NF-kB activity. ICP0 also binds to the endocytosis adaptor CIN85 and along with Cbl promotes internalization of the viral entry receptor Nectin-1. This is a mechanism to promote progeny virus spread to uninfected cells. (B): The tegument protein $\mathrm{U}_{\mathrm{L}} 46$ blocks STING and TBK1, which prevents stimulation of interferon-regulated genes. ICP34.5 and $\mathrm{U}_{\mathrm{S}} 11$ are also involved in blocking TBK1, emphasizing the importance of blocking TBK1 activity during HSV-1 infection. (C): The autophagy pathway is blocked during HSV-1 infection through binding of ICP34.5 to Beclin-1, thus preventing maturation of the autophagophore to an autophagosome. ICP0 has also been found to cause downregulation of p62 and OPTN proteins during infection, which may also serve as another mechanism of blocking selective autophagy. It has also been found that the protein encoded by $\mathrm{U}_{\mathrm{L}} 12.5$ causes depletion of mtDNA during infection, which causes damage to mitochondria. (D): HSV-1 prevents host translational shutoff from occurring during infection. One mechanism is through ICP34.5 binding to both PP1a and eIF $2 \alpha$, causing dephosphorylation of eIF $2 \alpha$ and preventing shutoff of translation. HSV-1 also encodes vhs, which is a viral RNase that degrades AU-rich element (ARE) containing mRNAs. It has also been shown that vhs prevents the formation of cytoplasmic stress granules (SGs) during infection, which contain dsRNA that would otherwise cause PKR activation. HSV-1 also encodes $U_{S} 11$, which blocks PKR, thus blocking host translational shutoff and innate immunity activation, as well as blocking PKR and PACT-induced activation of RIG-I during infection.

\section{2. $U_{L} 46$ and $U_{L} 47(V P 11 / 12$ and $V P 13 / 14)$}

The HSV-1 genes $\mathrm{U}_{\mathrm{L}} 46$ and $\mathrm{U}_{\mathrm{L}} 47$ were first described to encode proteins that modulate $\mathrm{U}_{\mathrm{L}} 48$ function [153-155]. It was then determined that UL46 encodes the phosphoprotein, VP11/12, and $U_{L} 47$ encodes the phosphoprotein VP13/14, which are also glycosylated, and both are non-essential in cell culture, although they have roles in enhancing the transactivation of viral genes by VP16 [156-161]. It was also found that $\mathrm{U}_{\mathrm{L}} 47$, but not $\mathrm{U}_{\mathrm{L}} 46$, deletion mutants of HSV-1 demonstrated a significant defect in alpha-TIF $\left(\mathrm{U}_{\mathrm{L}} 48\right)$-mediated expression of the TK gene, suggesting a supportive role in transcription of viral genes [161]. Homologs of $U_{L} 46$ and $U_{L} 47$ have been identified in multiple alphaherpesviruses, in- 
cluding pseudorabies virus, HSV-2, Marek's disease virus, and VZV [153,157,162-164]. Homologs of $\mathrm{U}_{\mathrm{L}} 47$ have been found in bovine herpesvirus and equine herpesvirus, and $\mathrm{U}_{\mathrm{L}} 47$ has been found to be abundantly expressed in the viral tegument $[153,165,166]$. T cell responses specific to $\mathrm{U}_{\mathrm{L}} 46$ and $\mathrm{U}_{\mathrm{L}} 47$ have both been found in patients with intraocular HSV-1 infection [167].

VP11/12 has since been found to be tyrosine phosphorylated during HSV-1 infection in multiple lymphocyte cell types but not in epithelia or fibroblasts, and this was found to at least partly be due to the activity of lymphocyte-specific Src family kinase, Lck [168]. It was then found that HSV-1 infection increases the amount of phosphorylation of Lck at Y394 in Jurkat T cells, which occurred in a $\mathrm{U}_{\mathrm{L}} 46$-dependent manner [169]. Moreover, it was found that the Akt pathway is activated through the PI3 kinase, and $\mathrm{U}_{\mathrm{L}} 46$ was found to be involved in the activation of this pathway in HSV-1-infected HEL fibroblast cells (Figure 2B) $[170,171]$. VP11/12 was then found to bind to the Src family kinases Grb2, p85, and Shc, thus leading to Akt activation in a T cell lymphoma cell line, and in support of this, it was found that infection with $\mathrm{U}_{\mathrm{L}} 46$-deficient viruses in HFFs led to reduced Akt activation as compared to infection with wild-type virus [163,172]. In neuronal cells, it was also found that VP11/12 was necessary but not sufficient during infection to induce the phosphorylation and activation of the small GTPase Dynamin 2 [173]. $\mathrm{U}_{\mathrm{L}} 46$ has also been found to interact with the small GTPase Rab27a during HSV-1 infection of oligodendrocytes, as well as with $\mathrm{gH}$ and $\mathrm{gD}$, but the significance of these interactions has not yet been investigated farther than reduced viral growth and infectivity in Rab27a-depleted cells [174].

It was demonstrated that HSV-1 infection of T cells led to the phosphorylation and degradation of adaptor complex protein Dok-2, which is involved in T cell regulation $[175,176]$. The degradation of Dok-2 occurred in a $\mathrm{U}_{\mathrm{L}} 46$-dependent manner, but the significance of this degradation has not yet been fully elucidated beyond a potential immune evasion strategy [177]. It was also recently found that $\mathrm{U}_{\mathrm{L}} 46$ works with $\mathrm{Us}_{3} 3$ to activate mTORC1 in fibroblasts, which supports virus growth [178]. Further supporting a role for $\mathrm{U}_{\mathrm{L}} 46$ in both immune evasion and viral growth was the observation that $\mathrm{U}_{\mathrm{L}} 46$ interacts with the DNA sensor STING (STimulator of INterferon Genes) at its C-terminus, which prevents the induction of interferon stimulatory genes and blocks type I interferon response to infection in fibroblasts (Figure 2B) [179]. Furthermore, it was found that $\mathrm{U}_{\mathrm{L}} 46$ binds to TBK1, TANK-binding kinase 1 , at its N-terminus and this was found to also play a role in blocking the interferon pathway in fibroblasts (Figure 2B) $[179,180]$. In support of this, the pseudorabies homolog of $\mathrm{U}_{\mathrm{L}} 46$ was found to interact with STING [181]. Together, these results show strong evidence for a role of $\mathrm{U}_{\mathrm{L}} 46$ in blocking innate immune responses to HSV-1 infection.

Less work has been done to characterize $\mathrm{U}_{\mathrm{L}} 47$ or its gene product, VP13/14. It has been found that VP13/14 is involved in nuclear egress through interactions with the viral proteins UL34, UL31, and $\mathrm{U}_{\mathrm{S}} 3$ [182]. $\mathrm{U}_{\mathrm{L}} 47$ was observed in the nucleus of infected cells during early times post-infection, which is consistent with the interaction of $U_{L} 47$ with $\mathrm{U}_{\mathrm{L}} 48$, but was also detected in the cytoplasm at late times post-infection [183-185].

$\mathrm{U}_{\mathrm{L}} 47$ has also been found, with ICP27, to regulate mRNA processing and transport by redistributing polyadenylate binding protein (PABP) to the nucleus during infection of HeLa cells, further supporting a role for $\mathrm{U}_{\mathrm{L}} 47$ in modulating post-transcriptional processing of mRNAs [186]. $U_{\mathrm{L}} 47$ has also been implicated in the regulation of the viral RNase vhs, which is discussed later in this review [187]. More work is needed to characterize fully the role of VP13/14 during HSV-1 infection, but so far it seems that $\mathrm{U}_{\mathrm{L}} 47$ facilitates viral infection by supporting viral gene transcription, mRNA processing, and transport.

\section{3. $U_{L} 49(V P 22)$}

VP22 is a 301-aa tegument protein (Figure 3) that is encoded by the $\mathrm{U}_{\mathrm{L}} 49$ gene [188], and has been associated with multiple functions during infection, which result from its interactions with host and cellular factors [189]. It localizes in multiple areas of the cell 
depending on the time point of the infection, even though its functional role is not always clear.

Early during infection, VP22 is mostly in the cytoplasm but eventually accumulates in the nucleus. VP22 is phosphorylated after entry into the cell, and this has been suggested to trigger its translocation into the nucleus [190,191]. Inside the nucleus, VP22 may be involved in modulation of nucleosome deposition and repression, which will affect virus life cycle progression [192]. VP22 may also have a function in the nucleolus, since it localizes there during early infection, even though it is not required for chromatin marginalization and HSV-1 replication [193].

VP22 associates with ICP0 in the nucleus, and its overexpression affects the transcription of gC and TK1 in the nucleus, suggesting that VP22 affects transcription though an interaction with ICP0 [194]. VP22 regulates the proper subcellular localization of VP16, VP26, ICP0, ICP4, ICP27, and Hsc-70 in infected cells [195]. These proteins localize to the nucleus early during infection and are then translocated to the cytoplasm later in infection. This translocation relies on specific dileucine motifs on VP22 [195].

VP22 also seems to be required for the expression of the vhs RNase, suggesting that expression of vhs in the absence of VP22 is lethal [196]. VP22-null mutants accumulate spontaneous secondary mutations in the $\mathrm{U}_{\mathrm{L}} 41$ (vhs) gene, therefore VP22 and vhs may have competitive functions [187]. There is a protein synthesis defect in the absence of VP22, which can result in a compensatory frameshift mutation in vhs. Mechanistically, VP22 and vhs interplay functionally at the level of accumulation and translation of viral mRNAs, indicated by the decrease in mRNA levels and polysome assembly when VP22 is absent. This phenotype can be rescued by the abovementioned complementary mutations in vhs [197]. VP22 is required for optimal protein synthesis at late times during infection, and the accumulation of $\mathrm{gE}, \mathrm{gD}$, and vhs mRNAs during early infection [198].

VP22 is not required for the accumulation of other tegument proteins, for virion assembly, or productive HSV-1 replication, but the size of the plaques of VP22 mutant HSV-1 strains (lacking the C-terminus) are smaller than the wild type (WT). Therefore, VP22 is probably required for efficient spread [191]. This effect may stem from the multiple interactions of VP22 as a tegument protein at sites of HSV-1 cytoplasmic envelopment, namely the TGN membranes [199]. Optimal packaging of VP22 in virions requires the amino acids 43-86, which facilitate localization of the protein to the TGN [200], but the exact order of VP22 packaging may be flexible. Overexpression of VP22 after infection with a recombinant HSV-1 that has two VP22 copies, resulted in 2-3-fold higher incorporation of VP22 into nascent virions [201]. In any case, the TGN VP22 mediates proper cytoplasmic envelopment as it interacts with the cytoplasmic tail of $\mathrm{gD}$, therefore bridging the viral capsid and the envelope (Figure 3) [202]. Additionally, VP22 interacts with $\mathrm{U}_{\mathrm{L}} 16$ and deletion of $\mathrm{U}_{\mathrm{L}} 16$ results in dramatic reductions of VP22 in released virions [203].

Furthermore, it interacts with gE [204,205]. Deletion of VP22 results in reduced amounts of ICP0, $\mathrm{gE}$, and $\mathrm{gD}$ in the extracellular infectious virions, whose number is also reduced [206]. Additionally, VP22 bridges a complex between $\mathrm{gE} / \mathrm{gI}$ and $\mathrm{gM}$, which is selective in its formation, since it does not include VP16, a close partner of VP22 [207]. This VP22/gE/gI/gM complex also recruits ICP0 in a VP22-dependent fashion. None of those proteins is absolutely required for the formation of a subcomplex; however, optimal complex formation results in efficient virus formation (Figure 3) [208].

One important aspect of VP22 is its involvement in cytoskeleton reorganization. VP22 requires microtubule reorganization for its translocation to the nucleus [209], and it also affects the reorganization and polymerization of the microtubules late during infection, suggesting a late trafficking role [185]. This occurs independently of virus replication or other viral factors $[209,210]$. 


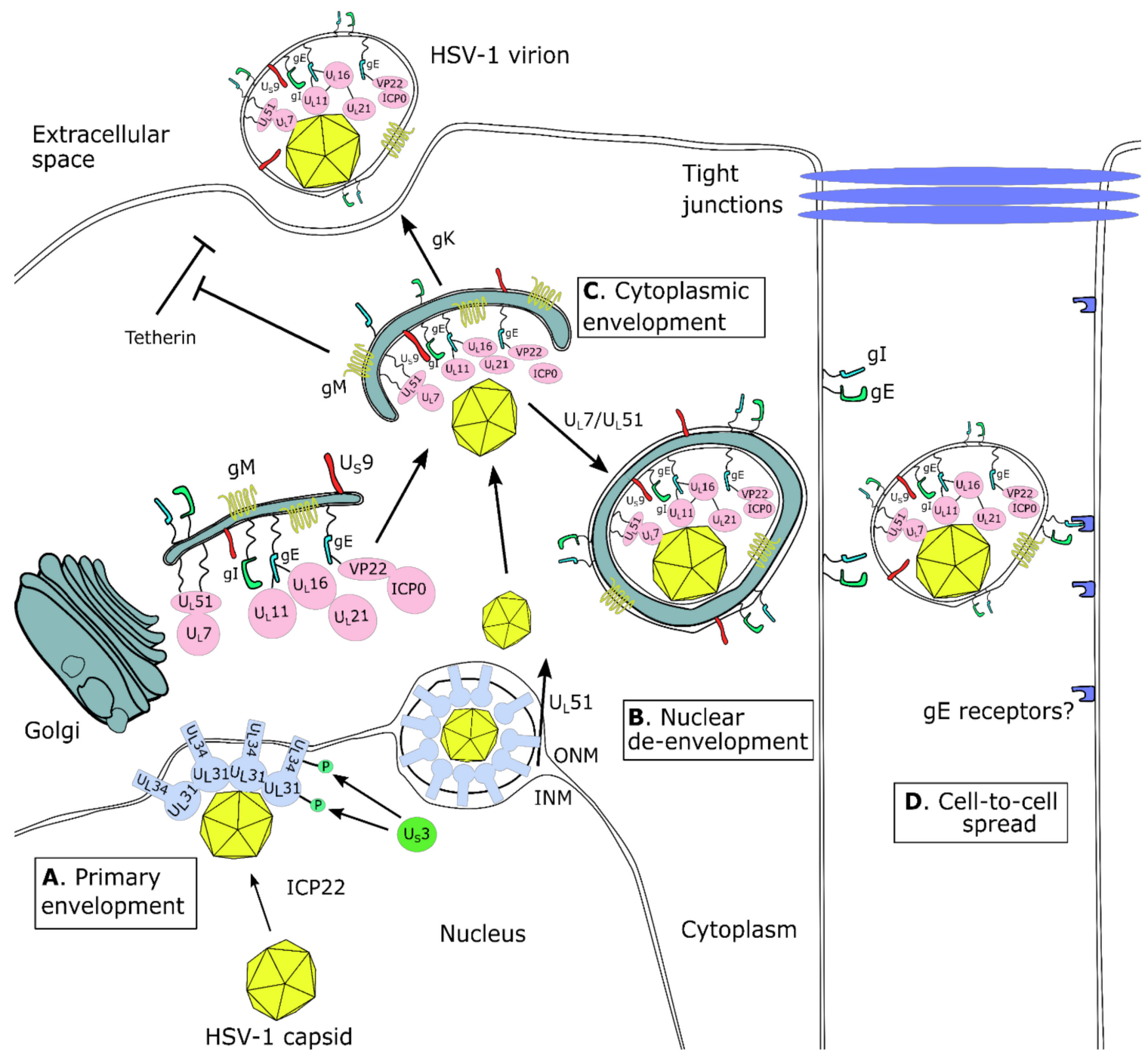

Figure 3. HSV-1 egress and relevant non-essential proteins. Essential proteins are not depicted. (A) $U_{L} 31$ and $U_{L} 34$ form the Nuclear Egress Complex (NEC), which drives the vesiculation of the inner nuclear membrane (INM) and primary envelopment of HSV-1 capsids. (B) Nuclear de-envelopment is mediated by $\mathrm{U}_{\mathrm{L}} 51$. Tegument and envelope proteins assemble in a complex network on membranes derived from the trans-Golgi network. (C) HSV-1 capsids undergo cytoplasmic envelopment in a process regulated by multiple non-essential proteins, which involves functional redundancy. After cytoplasmic envelopment, enveloped virions are sorted to the extracellular space, which requires gK. HSV-1 release from the cell membrane can be inhibited by tetherin, which is counteracted by gM. (D) Depending on cell type (e.g., polarized epithelial cells), enveloped virions can disseminate through cell-to-cell spread, in a process that requires $U_{L} 7 / U_{L} 51$ for sorting of virions towards parts of the membrane that contain $\mathrm{gE} / \mathrm{gI}$. Virions may spread to adjacent cells by binding to $\mathrm{gE} / \mathrm{gI}$ receptors in adjacent cells. However, such receptors are unknown.

VP22 localization is also affected by motor proteins, like the actin-associated motor protein non-muscle myosin IIA (NMIIA). Inhibition of the ATPase activity of NMIIA impaired the perinuclear vesicular pattern of VP22 and the release of virus into the extracellular space, but it did not affect the cell-associated virus. VP22-containing particles line up along NMIIA-containing filaments that run through protrusions, which can emanate from infected cells [211]. The interactions of VP22 with the cytoskeleton probably affect CD1d-mediated activation of natural killer T (NKT) cells. CD1d is an MHC class I-like molecule that mediates self and microbial lipid presentation to NKT cells. HSV-1 can inhibit CD1d-mediated antigen presentation to NKT cells by suppressing CD1d recycling to the cell surface. VP22 is required for this inhibition of CD1d recycling, which probably occurs 
because of the reorganization of the cytoskeleton that VP22 promotes, which consequently affects CD1d recycling to the plasma surface [212].

An important host factor that VP22 interacts with is the AIM2 inflammasome, promoting the evasion of AIM2-dependent inflammasome activation during infection. The AIM2 inflammasome is normally activated by DNA, which would be available during HSV-1 infection since the HSV-1 nucleocapsid has been reported to be degraded in the cytoplasm [213]. However, HSV-1 infection induces AIM2-independent inflammasome activation, which is inhibited by VP22. VP22 interacts with AIM2 and prevents its oligomerization, which is the first step in AIM2 inflammasome activation. Mice that lack AIM2 can support infection of a VP22-null HSV-1 [214]. Considering that VP22 can move between cells [215], this would be an efficient manner to block inflammatory responses in uninfected cells adjacent to the infection.

Another property of VP22 is that it can be transported intercellularly using a Golgiindependent mechanism, which involves the actin cytoskeleton since it is sensitive to cytochalasin D [215]. The importance of VP22 in spread is most evident in animal models. VP22 is required for efficient development of corneal lesions in mice following ocular inoculation (Figure 4) and it is also important for neurovirulence, through two possible mechanisms [206]. First, two dileucine motifs of VP22 (at positions 235-236 aa and 251-252 aa) are required for spread of viral antigens in the mouse brain and efficient virulence. These two motifs have been associated with proper cytoplasmic localization of other viral proteins, and VP22 may mediate neurovirulence though that function [195]. Second, a VP22 mutant HSV-1 exhibits impaired viral replication (about 1000-fold) and spread in the brains of infected mice, supporting the importance of VP22 for virus spread in neurons [195]. VP22 may exert its proviral effect in neuronal spread through blocking of AIM2-dependent inflammasome activation, as explained above. Infection of AIM2 ${ }^{-/-}$mice with a VP22-null HSV-1 results in 3 logs higher viral yield than infection of AIM2 ${ }^{+/+}$mice, suggesting that VP22 promotes neuronal spread by inhibiting an AIM-2-dependent host response against HSV-1 infection [214].

The role of VP22 in spread is also used for the transport of viral RNAs during infection to adjacent non-infected cells, but this function can also be utilized for the delivery of products, such as chimeric polypeptides [216]. For example, a chimera consisting of VP22 linked to p53 can spread between cells and accumulate in recipient cell nuclei, while inducing apoptosis in p53-negative osteosarcoma cells [217]. There have been other chimeras that are described in the literature, but the potential of this strategy is not definitive. Not all types of cargo can be carried by VP22, and in vitro data may not translate well in animal models [218-222]. Additionally, VP22 conjugated cargo may be transported, but it might not be functional [223]. These conflicting results suggest that VP22 is not ideal for carrying all proteins, either due to inefficient transport of the chimeras or structural effects of the chimera on VP22. After mapping the functional domains of VP22, engineered versions of VP22 with higher transport efficiency could be investigated [189]. Nonetheless, VP22 chimeras are a promising tool and could be used in cancer, gene therapy, and vaccines. Notably, we have not detected VP22 in $\mathrm{CD}^{+} 3^{+} \mathrm{EVs}_{\mathrm{s}}$ or ESCRT ${ }^{+}$EVs derived from HSV-1 infected cells, suggesting that the intercellular transfer of VP22 does not depend on light extracellular vesicles (lighter than virions) derived from infected cells [224,225].

A potential avenue for utilizing VP22 transport in cancer therapy involves the introduction into target cells of a nontoxic drug and an enzyme that can convert to it to toxic. For example, introduction in cancer cells of the thymidine kinase (TK) gene and ganciclovir $(\mathrm{GCV})$ results in phosphorylation of GCV, turning it into a nucleoside analogue that kills cells by inhibiting chain extension during deoxyribonucleic acid synthesis. Cytotoxicity is also observed in adjacent cells of a tumor. However, the levels of prodrug that need to be administered to kill adjacent cells in a solid tumor end up being toxic for the patient. These problems of efficient TK and GCV delivery can be resolved through VP22 [226]. VP22 can enhance intercellular trafficking of TK and can amplify the killing effect of the TK/GCV combination, making the fusion of TK and VP22 an attractive candidate for cancer 
therapy [227]. Another anticancer treatment is the use of the bacterial enzyme cytosine deaminase (CD) with the prodrug 5-fluorocytosine (5-FC), which is converted by CD to the highly toxic 5-fluorouracil. The efficacy of this combination can be enhanced through fusion of CD and VP22. The CD-VP22 fusion has a higher cytotoxicity in mouse models when compared to administration of CD alone [228,229].

VP22 could also be used in the context of gene therapy as a carrier [230]. For example, intraocular administration in mice of an adenoviral vector carrying VP22 fused to GFP showed a dramatic increase in the number of CNS neurons expressing GFP versus when administering an adenovirus with just GFP [231]. Other potential uses of VP22 to enhance adenovirus-based gene transfer have been noted in the literature [232]. VP22 can also enhance DNA vaccine protection against $P$ seudomonas aeruginosa in mice [233]. It is possible to fuse VP22 to other antigens of interest inside a DNA vaccine and that can enhance antigen-specific responses and antitumor effects [234].

\section{4. $U_{S} 1(I C P 22)$}

The $\mathrm{U}_{\mathrm{S}} 1$ immediate-early gene product, ICP22, is a 420-amino-acid protein. Mutant viruses lacking ICP22 display reduced virus yields in some cell lines, including primary human and rodent cell lines, but not in others, such as Vero (African green monkey) and HEp-2 cells (human epithelial), implying cell type-dependent effects [19,20,235-242]. Using different models of infection in mice and guinea pigs, a virus deleted of ICP22 caused reduced virulence and displayed reduced replication during an acute ocular infection and reduced neurovirulence $[238,243-246]$. Homologs of ICP22 are found in other herpesviruses, though the importance of ICP22 in infection seems to differ between viruses [247-251] ICP22 is guanylylated, adenylylated, and is phosphorylated by $\mathrm{U}_{\mathrm{L}} 13$ and $\mathrm{U}_{\mathrm{S}} 3$ [252-256]. Phosphorylation of ICP22 at tyrosine 116 has been found to be important for ocular infection, affecting virulence, but the kinase responsible has not yet been specified [245].

ICP22 contains two nuclear import signals and has been implicated in viral gene expression $[238,239,257,258]$. Particularly, the carboxyl-terminal domain (CTD) of ICP22, in conjunction with the viral $\mathrm{U}_{\mathrm{L}} 13$ protein kinase, was found to enhance the synthesis of a subset of late $\left(\gamma_{2}\right)$ proteins exemplified by the products of the $U_{L} 38, U_{L} 41$, and $U_{S} 11$ genes (Figure 1C). ICP22 and the $U_{L} 13$ protein kinase mediate the activation of cdc2 and degradation of its partners, cyclins A and B. Cdc2 and its new partner, the viral DNA polymerase accessory factor $\left(\mathrm{U}_{\mathrm{L}} 42\right)$, bind topoisomerase II $\alpha$ in an ICP22-dependent manner (Figure 1C) [259-262]. Although topoisomerase II is required for viral DNA synthesis, ICP22 is not, suggesting that the ICP22/topoisomerase II interplay has another role during HSV-1 infection. Indeed, topoisomerase II appears to be required for untangling concatemeric DNA progeny for optimal transcription of late genes.

Regarding the role of $\mathrm{U}_{\mathrm{L}} 13$ in the abovementioned complex, it was found that ICP22 and $\mathrm{U}_{\mathrm{L}} 13$ are involved in a common pathway that alters RNAP II phosphorylation, and in some cell lines, this change promotes viral late transcription, and also involves $\mathrm{U}_{\mathrm{S}} 1.5$, a shorter gene encoded from the $\mathrm{U}_{\mathrm{S}} 1 \mathrm{ORF}$ (Figure 1C) [263-266]. This ICP22/ $\mathrm{U}_{\mathrm{L}} 13$-mediated phosphorylation of RNAP II resulted in an "intermediate" electrophoretic mobility between that of hyperphosphorylated (RNAP IIo) and hypophosphorylated (RNAP IIa) states [267]. Furthering this work, it was found that $\mathrm{U}_{\mathrm{L}} 13$ and the C-terminus of ICP22 are both required for RNAP II phosphorylation [267-269]. In cells infected with mutants from which $U_{L} 13$ had been deleted, ICP22 fails to aggregate in the nuclear structures containing nascent DNA, ICP4, RNA polymerase II, and other factors, implying a role of this $\mathrm{U}_{\mathrm{L}} 13$-mediated phosphorylation in viral late gene expression (Figure 1C) [270-273].

ICP22 was also found to bind the cyclin-dependent kinase 9 (cdk9) but not cdk7, and this complex in conjunction with viral protein kinases $\left(U_{L} 13\right.$ and $\left.U_{S} 3\right)$ phosphorylates the carboxyl terminus of RNAP II. The primary function of cdk9 and its partners, the cyclin $\mathrm{T}$ variants, is in the elongation of RNA transcripts, although functions related to the initiation and processing of transcripts have also been reported. Cdk9 was found to be important for optimization of the expression of genes regulated by ICP22. Therefore, 
one function of cdk9 during HSV-1 infection may be to bring ICP22 into the RNAP II transcription complex [274-277]. In support of these findings, it was reported that ICP22 binds to positive transcription elongation factor $\mathrm{b}(\mathrm{P}-\mathrm{TEFb})$ and to RNAP II, and along with cdk9 they could suppress the expression of host genes, offering an advantage to the virus $[278,279]$. In fact, ICP22 represses transcription from all classes of viral genes but selectively upregulates expression of some late $(\gamma 2)$ genes [280,281]. Thus, ICP22 may be important to either repress or activate viral genes at different stages of the viral life cycle.

Besides its functions in viral gene transcription, a regulatory role has been proposed for ICP22 that involves the differential expression of two transcripts produced by the $U_{S} 3$ open reading frame. The $U_{S} 3$ gene was reported to encode two proteins. In wild-type virus-infected cells, the predominant form is the full-length Us3. However, in ICP22-null virus-infected cells, a shorter form of $\mathrm{U}_{\mathrm{S}} 3$ is produced that initiates from methionine 77 and has been named $U_{S} 3.5$ [282]. Like $U_{S} 3$, the $U_{S} 3.5$ mediates the phosphorylation of HDAC1, HDAC2, the protein kinase A regulatory $\mathrm{II} \alpha$ subunit (PKA RII $\alpha$ ), and the $\mathrm{U}_{\mathrm{L}} 31$ protein. Additionally, both kinases cofractionate with mitochondria. However, the $U_{S} 3.5$ failed to block apoptosis (a well-established role of $U_{\mathrm{S}} 3$ ) and does not enable efficient release of virus particles from nuclei. Thus, the two proteins differ in the range of functions they exhibit [103].

Another role that has been attributed to ICP22 is involved in the host cell chaperone machinery by facilitating the formation of virus-induced chaperon-enriched (VICE) domains in the nucleus of some infected cells. Recent studies suggested that ICP22 mimics a cellular type II J protein, which is a co-chaperone in the nucleus [283-285]. The VICE domains are usually formed adjacent to the viral replication compartments, they contain several host chaperones (Hsp70, Hsp40, Hsp90), proteasomal components, ubiquitinated proteins, and at least one viral protein. These domains are hypothesized to play a role in protein quality control and remodeling, and during infection, they may participate in the formation of viral replication compartments and transcriptional regulation. VICE domains may also allow for correct folding of proteins participating in macromolecular assemblies. VICE domains may have a greater role in certain cell lines, where ICP22 expression is essential [273,286-290].

ICP22 was also found to form a complex with the HSV-1 proteins $U_{L} 31, U_{L} 34, U_{L} 47$, and $U_{S} 3$ (Figure 3). These proteins, as discussed elsewhere, are important for viral egress through the nuclear membrane. ICP22 colocalizes with $\mathrm{U}_{\mathrm{L}} 31$ and $\mathrm{U}_{\mathrm{L}} 34$ at the nuclear membrane in WT virus-infected cells. In $\mathrm{U}_{\mathrm{L}} 31$-null virus-infected cells, targeting of ICP22 to the nuclear membrane is inhibited. In ICP22-null virus-infected cells, $\mathrm{U}_{\mathrm{L}} 31$ and $\mathrm{U}_{\mathrm{L}} 34$ mis-localized in the ER and the nuclear membrane, and significantly reduced the numbers of primary enveloped virions that were observed in the perinuclear space, although capsids accumulated in the nuclei. These data suggest that ICP22 plays a role in HSV-1 primary envelopment by interacting with the nuclear egress complex [291].

More recently, roles for ICP22 in combating the immune system have been proposed. The T cell co-stimulatory molecule CD80 was found to be downregulated in DCs in a manner dependent on ICP22 binding to the CD80 promoter, which seems to limit the pathogenesis of the virus as well as delaying the immune response to infection [292-294]. Recent studies with HSV-2 and transfection assays have found that ICP22 may be important in blocking type I IFN responses during infection [295]. Additionally, ICP22 may regulate host E3 ubiquitin ligases. Cumulatively, the findings regarding ICP22 are that it is important for the expression of the late $\left(\gamma_{2}\right)$ class of viral genes, formation of viral replication compartment and VICE domains in the nucleus, binding to host transcripts thereby altering host responses to the virus, and even by facilitating nuclear egress of viral capsids. 


\section{Host Evasion Factors}

\subsection{RL1 or $\gamma 134.5$ (ICP34.5)}

The HSV-1 $\gamma_{1} 34.5$ gene product was first described in 1986 [296,297], and HSV-1 and HSV-2 are the only members of the alphaherpesviruses expressing ICP34.5 [298,299]. Deletion of the ICP34.5 gene abolished the capacity of the virus to spread from peripheral mucosal sites to the central nervous system (CNS) or replicate in the CNS, and diminished the capacity of the virus to replicate at mucosal sites and, subsequently, establish latency, or be able to be reactivated ex vivo [300]. In support of this, an ICP34.5-null virus displayed reduced neurovirulence following intracerebral inoculation into mice [298,299,301-307]. Furthermore, in an ocular model of infection, ICP34.5-null virus did not cause corneal disease [308]. In mouse embryonic dorsal root ganglia (DRG) three-dimensional cultures for HSV-1 latency, a virus with a deletion in ICP34.5 rendered the virus incapable of reactivation, even though the virus was clearly able to replicate and persist in a quiescent form in the DRG neurons [309].

The requirement of ICP34.5 for viral growth is cell type and status dependent, including an inability to replicate in non-dividing cells [303,310-312]. The ICP34.5 protein contains a domain homologous to GADD34/MyD116, which functions during growth arrest or DNA damage [313-316]. In cell culture, this domain of ICP34.5 was shown to be required for preventing the shutoff of host translation through the reversal of phosphorylation of eukaryotic translation initiation factor alpha (eIF2 $\alpha$ ), which occurs in a PKR-dependent fashion (Figure 2D) [317-322]. It was later found that ICP34.5 associates with the host protein phosphatase $1 \alpha$ via its C-terminus and redirects it to dephosphorylate eIF2 $\alpha$ (Figure 2D) $[317,318,323]$.

In interferon (IFN)- $\alpha / \beta$ receptor knockout mice, the ICP34.5-null virus showed a rescue to near wild-type replication levels in the trigeminal ganglia, and this sensitivity to IFN- $\alpha / \beta$ was found to occur in a manner dependent on the RNA sensor protein kinase $R$ (PKR) $[135,324,325]$. It was also found that mouse embryonic fibroblasts (MEFs) infected with an ICP34.5-null virus induced higher expression of innate immunity genes and phosphorylation of the transcription factor IRF3, which was partially dependent on TANKbinding kinase 1 (TBK1) binding (Figure 2B) [326]. The binding site of TBK1 to ICP34.5 was found to be dispensable for blocking IRF3 phosphorylation, though ICP34.5 was still demonstrated to be important for this function [327].

Another major role for the ICP34.5 protein is blocking autophagy through its interaction with Beclin-1, which has been observed largely in mouse embryonic fibroblasts (MEFs) (Figure 2C) [321,328,329]. Viruses lacking the Beclin-binding domain (BBD) of ICP34.5 were attenuated [328]. Mice infected with the mutant virus missing the BBD of ICP34.5 replicated less in brain and corneal tissue, but the BBD was found to be dispensable for reactivation [330]. The control of autophagy by ICP34.5 was also implicated in preventing MHC-class I antigen presentation of $\mathrm{gB}$ from HSV-1-infected macrophages to $\mathrm{CD} 8^{+} \mathrm{T}$ cells through autophagy [331]. In support of this, the BBD of ICP34.5 was found to be important to antagonize autophagy and prevent MHC-class II antigen presentation in dendritic cells (DCs) [332]. Consistently, there seemed to be an increase in $\mathrm{CD}^{+} \mathrm{T}$ cell responses, including IFN- $\gamma$ and IL-2 production in mice infected with HSV-1 lacking the ICP34.5 BBD [330]. However, in MEFs lacking the gene Atg5, which is essential for autophagy, the infection with ICP34.5 deleted for the BBD was not rescued compared to wild-type MEFs and no improvement in virus replication was noticed $[333,334]$. This suggests that the primary role of ICP34.5 is to counteract PKR activation rather than xenophagy. In support of this, the growth of ICP34.5-deficient virus was completely rescued in $\mathrm{PKR}^{-/-}$MEF cells. The discrepancies in the importance of the BBD and the fact that certain dendritic cell lines and neuroblastoma cells actually exhibiting higher autophagy activation when infected with an ICP34.5-null virus highlight the cell type-dependent role of ICP34.5 [321,328,333,335-337]. It was recently described that the expression of the ICP0 protein of HSV-1 was not sustained in the ICP34.5-deleted virus, which complicates our understanding of cellular effects by this virus [327]. More work is needed to clarify how cell type and cell status influences 
infection with ICP34.5-mutant viruses, and more specifically the ways in which ICP34.5 is able to modulate autophagy in different cells.

\section{2. $U_{S} 12(I C P 47)$}

Infection of fibroblasts with HSV-1 renders the cells resistant to lysis by $\mathrm{CD} 8^{+}$cytotoxic T lymphocytes (CTLs), which normally recognize cell surface MHC I proteins presenting viral antigens. ICP47 can block the transport of MHC I proteins to the surface, and in this way inhibits lysis of infected cells by CTLs [338]. This explains why using ICP47 in a vaccine vector cannot confer protective immunity in vivo, since it prevents MHC I CTL induction [339]. To prevent the presentation of MHC I molecules on the cell surface, ICP47 binds to the transport-associated with antigen processing (TAP) factor. TAP mediates the transport of peptides destined for presentation by MHC I from the cytosol to the ER [340]. ICP47 binds with high affinity to the substrate-binding site of TAP, preventing the binding of other peptides [341,342], and this binding is species specific, since binding to murine TAP is much weaker [343,344]. This suggests that mice are not the optimal animal model to study the $\mathrm{CD}^{+} \mathrm{T}$ cell protective effect of ICP47, but pigs, dogs, or monkeys appear more suitable [345]. Alternatively, recombinant HSV-1 strains that contain murine MHC I complex-binding proteins can be used, and they effectively restrict MHC I antigen presentation in murine models. Work on such models has demonstrated that preventing MHC I antigen presentation increases neurovirulence of HSV-1 since viral entry, replication, and survival in the CNS is possible [346].

Preventing MHC I presentation can also be used to enhance the potency of oncolytic mutant strains, such as those based on ICP34.5-null mutants [347,348]. When using oncolytic viruses for cancer immunotherapy, there are issues with the immunogenicity against viral vectors that carry antigens and the memory response that arises after repeated injection of the vector during prime-boost regimens. CTL responses against the vectors prevent build-up of an immune response against the antigen of interest, which in the case of cancer immunotherapy is a peptide that is expressed in tumors. To solve this issue, a tumor peptide can be fused with part of the adenovirus 19K-derived leader sequence (MRYMILGLLALAAVCSA), which is an ER-targeting sequence, so that when expressed in APCs, the fusion product bypasses TAP and traffics to the ER. In the ER, the tumor antigen can be trimmed by aminopeptidases [349], loaded on MHC I molecules, and presented on the cell surface in a TAP-independent manner [350]. In parallel, $\mathrm{U}_{\mathrm{S}} 12$ can be expressed from the viral vector and its expression restricts TAP and TAP-dependent MHC I presentation. In the end, viral vector peptides will not be presented through the TAP pathway and the immunogenicity against the vector will be restricted [351].

This protective role of ICP47 against CD8 ${ }^{+} \mathrm{T}$ cells is responsible for enhancing HSV-1 neuropathology in vivo. While an ICP47-null virus and WT replicate similarly in corneal epithelial tissues, the ICP47-null virus causes little to no neurologic disease and encephalitis [352]. Mice depleted of T cells can support WT levels of neurovirulence, but mice survival is decreased after exogenous delivery of $\mathrm{CD} 8^{+} \mathrm{T}$ cells [353], suggesting that CTLs can control HSV-1-associated disease. Presentation of HSV-1 peptides to CTLs through TAP is inhibited by ICP47, thus absence of ICP47 restricts neurovirulence whereas absence of TAP does not [354]. Additionally, TAP expression in the brain of infected mice is increased, suggesting that it has a host defense role. Infection with an ICP47-null HSV-1 virus does not trigger an increase of TAP, likely because this virus does not invade the brain [354].

\section{Nucleic Acid Metabolism and Endonucleases}

\section{1. $U_{L} 2, U_{L} 12, U_{L} 12.5, U_{L} 50$}

HSV-1 has been shown to encode multiple proteins for the metabolism of nucleic acids in the host cell. One such protein is encoded by $\mathrm{U}_{\mathrm{L}} 2$, and is the uracil DNA glycosylase, which is an important enzyme for removing uracil from DNA [355-359]. The uracil DNA glycosylase activity was described to be important in adult neurons for the replication of the viral genome [360,361]. In support of this, infection of mice with mutant viruses lacking 
uracil DNA glycosylase activity had significantly reduced viral load in peripheral and central nervous system tissues, as well as reduced reactivation from latency [361]. Later, $\mathrm{U}_{\mathrm{L}}$ 2-encoded uracil DNA glycosylase activity was found, with cellular factors and with the viral DNA polymerase, to participate in base excision repair coupled with DNA replication, supporting a role for $\mathrm{U}_{\mathrm{L}} 2$ during genome replication [362-365]. Moreover, $\mathrm{U}_{\mathrm{L}} 2$ nuclear localization was found to be important for efficient viral replication [366]. $\mathrm{U}_{\mathrm{L}} 2$ was found to be nonessential in cell culture [367]. There is a homolog for this protein in other herpes viruses [368-371].

HSV-1 also encodes an alkaline nuclease or deoxyribonuclease, which is a phosphoprotein with endo- and exonuclease activity, which localizes to the nucleus of infected cells [372-375]. The alkaline nuclease is encoded by the $\mathrm{U}_{\mathrm{L}} 12$ gene of HSV-1 [22,374,376379]. The $U_{L} 12$ gene of HSV-1 is highly related in sequence to proteins from other herpes viruses [380-383]. $\mathrm{U}_{\mathrm{L}} 12$ is not essential for viral DNA replication [384]. $\mathrm{U}_{\mathrm{L}} 12$ is important for viral capsids to egress from the nucleus [385,386]. It was found that cells infected with a mutant virus lacking $\mathrm{U}_{\mathrm{L}} 12$ released many particles with genomes incapable of undergoing new rounds of infection [387]. This increase in defective particles released in the absence of $\mathrm{U}_{\mathrm{L}} 12$ during infection was later found to specifically be due to the nuclease activity of the protein [388].

Mutant viruses lacking alkaline nuclease activity have reduced growth in cell culture, which may be due to a role for $\mathrm{U}_{\mathrm{L}} 12$ in processing viral DNA replication intermediates and packaging the DNA into the capsid [389-391]. More specifically, $\mathrm{U}_{\mathrm{L}} 12$ has been found with ICP8, the ssDNA binding protein of the virus, to mediate strand exchange during DNA replication through increased nuclease function by $\mathrm{U}_{\mathrm{L}} 12$ [392-394]. $\mathrm{U}_{\mathrm{L}} 12$ also seems to be involved in single-strand annealing during homologous DNA repair in infected cells, which also involves ICP8 as the single-strand annealing protein $[395,396]$. $\mathrm{U}_{\mathrm{L}} 12$ was also found to interact with components of the MRN (homologous recombination repair complex containing the proteins Mre11, Rad50, and Nbs1) in the nucleus during infection [397]. Together, these reports support a role for $\mathrm{U}_{\mathrm{L}} 12$ in the processing of viral DNA during infection.

The $\mathrm{U}_{\mathrm{L}} 12.5$ protein of HSV-1 has not been fully characterized, but its ORF is known to overlap that of $U_{L} 12$. $U_{L} 12.5$ lacks the first 126 aa of $U_{L} 12$, it retains the nuclease and the ICP8 binding activities of $\mathrm{U}_{\mathrm{L}} 12$, and was initially described as a capsid nuclease $[398,399]$. However, $\mathrm{U}_{\mathrm{L}} 12.5$ does not accumulate to high levels in the nucleus and cannot efficiently substitute for $\mathrm{U}_{\mathrm{L}} 12$ in promoting viral genome maturation [400]. Interestingly, the only known function of $U_{\mathrm{L}} 12.5$ seems to be related to mitochondria stress, as it has been found that mitochondrial DNA is eliminated early during HSV-1 infection in a $\mathrm{U}_{\mathrm{L}}$ 12.5-dependent manner, which also involves mitochondrial nucleases (Figure 2C) [401-403]. This role by $\mathrm{U}_{\mathrm{L}} 12.5$ in altering mitochondria stability during infection has not been further described, but it has been found to be dispensable for viral replication [404]. More work is needed to understand the role of $\mathrm{U}_{\mathrm{L}} 12.5$ during HSV-1 infection.

Another important protein involved in viral DNA replication is the protein encoded by $\mathrm{U}_{\mathrm{L}} 50$ of HSV-1. $\mathrm{U}_{\mathrm{L}} 50$ encodes a tegument protein, which is a deoxyuridine $5^{\prime}$-triphosphate nucleotidohydrolase (dUTPase), which is important for the synthesis of thymidine for DNA replication [153,405-408]. The dUTPase activity was found to be nonessential in cell culture; however, replication in the $\mathrm{CNS}$ of mice infected with a $\mathrm{U}_{\mathrm{L}} 50$-deleted virus was reduced, along with reduced neurovirulence and reactivation $[358,409,410]$. The dUTPase is phosphorylated by the viral kinase $\mathrm{U}_{\mathrm{S}} 3$ (see below), which seems to regulate the activity of this protein in a cell type-dependent manner, as well as specifically affecting the neurovirulence and replication competency of HSV-1 in the central nervous system [411-413]. There are homologs for $U_{L} 50$ in other members of the Herpesviridae family [164,380,381,414-417]. Recent reports studying homologs of the HSV-1 $\mathrm{U}_{\mathrm{L}} 50$ from EBV or using transfections of multiple $U_{L} 50$ homologs show the ability of $U_{L} 50$ to affect TLR1/TLR2-mediated immune responses and upregulate NF-KB activity [418-420]. While it is still not known how this pro-inflammatory effect by the HSV-1 dUTPase affects viral infection or pathogenesis, 
particularly as it relates to infection of different cell types, it is worth further investigation to understand the immunomodulatory capacity of HSV-1 and the viral dUTPase.

\section{2. $U_{L} 39$ and $U_{L} 40$ (RR1 and $\left.R R 2\right)$}

The holoenzyme of the viral ribonucleotide reductase (RR) is composed of two subunits, the large subunit, also known as ICP6, that is encoded by the $\mathrm{U}_{\mathrm{L}} 39$ gene and the small subunit encoded by the $\mathrm{U}_{\mathrm{L}} 40$ gene. HSV-1 RR converts ribonucleotide diphosphates to corresponding deoxyribonucleotides, allowing for virus replication, particularly in nondividing cells [421]. Both subunits of the RR are needed for enzyme activity [422], so a decrease in either subunit decreases RR activity. Knockdown of $U_{L} 40$ using siRNAs triggers a mild $(50 \%)$ decrease in plaque size and numbers [423]. Chemical inhibitors of the ribonucleotidase activity can lead to poor viral replication, depending on the cell type [371,424,425]. Dividing cells in S phase contain an elevated dNTPs pool and are capable of supporting replication of ribonucleotide reductase-deficient virus [421,426]. RR is required in non-replicating cells, such as neurons that have a reduced dNTPs pool (Figure 4A). This attribute makes an RR-deficient virus an attractive option for use in oncolytic therapy that targets malignant gliomas, since it generates a non-replicating virus in such tissues, giving a good safety profile [427].

HSV-2 $\mathrm{U}_{\mathrm{L}} 39$ has been associated with antiapoptotic functions [428]. HSV-1 $\mathrm{U}_{\mathrm{L}} 39$ mutants exhibit a 50\% reduction in protection from TNF $\alpha$ [429], which suggests that HSV-1 $\mathrm{R} 1$ is important for protection of HSV-infected cells from this death ligand, while the $50 \%$ efficiency suggests that other viral proteins contribute to this protection. Nonetheless, HSV-2 R1 interacts constitutively with caspase-8 and prevents its interaction with FADD, inhibiting TNF $\alpha$-mediated apoptosis [430].

The large subunit of the RR has been shown to contribute to ocular virulence in mice [431], but null mutants can produce lesions in a guinea pig model [432]. This has also recently been observed with a naturally occurring viral mutant in mice that were impaired in acute replication in the eyes and the trigeminal ganglia of mice, and also defective in establishing a latent infection and reactivation [433]. Interestingly, this mutant cannot inhibit caspase 8-induced apoptosis as wild-type virus [433], further supporting the relevance of the antiapoptotic effects of the RR for pathogenesis. An RR-deficient virus that exhibits impaired acute replication in the eyes and the trigeminal ganglia of mice is an attractive option for a herpes prophylactic vaccine, since it appears to confer protection against HSV-1 challenge post-immunization with an RR-deficient mutant [434].

\section{3. $U_{L} 41$ (vhs)}

It was first observed that HSV-1 caused the shutoff of host protein synthesis as early as 1978 [435-437]. This effect was later ascribed to the $\mathrm{U}_{\mathrm{L}} 41$ gene product of HSV-1, the virion host shutoff (vhs) protein, which is found in the viral tegument [438-442]. There are homologs for vhs in other alphaherpesviruses, such as HSV-2, varicella-zoster virus, equine herpesvirus, and pseudorabies, which is indicative of a conserved benefit for the virus to express this protein [443-445]. Although vhs is dispensable in cell culture, its absence in vivo leads to reduced viral pathogenicity and severe attenuation [135,441,446-450]. Vhs was first described to be able to block the accumulation of both host transcripts and all three classes of viral mRNAs [438-441,451-453]. However, it was later suggested that the activity of vhs is regulated late during infection by the viral proteins $\mathrm{U}_{\mathrm{L}} 47$, VP16, and VP22 and this is how it spares beta and gamma viral transcripts, although it downmodulates alpha gene transcripts [187,454-457]. Vhs blocks the accumulation of mRNAs due to its role as a viral RNase [452,458-460]. More specifically, vhs has been identified as an endoribonuclease, with sequence similarities to the FEN-1 family of nucleases, which are found in eukaryotes and archaebacteria and are involved in DNA replication and repair [460-462]. Vhs displays substrate specificity similar to that of RNase A and it cleaves at the 3' end of single-stranded cytidine or uridine residues [463]. A group of mRNAs targeted by vhs includes those with adenylate-uridylate (AU)-rich elements at the $3^{\prime}$ end. Several AU-rich mRNAs are stress 
response transcripts that are upregulated during HSV-1 infection, as they encode for hostile products, including type-I interferon-related products [457,464,465].

These mRNAs appear to be cleaved in ARE and deadenylated in a $3^{\prime}-5^{\prime}$ decay process occurring in a vhs-dependent manner, whereas the truncated $5^{\prime}$ domains may persist, and this is a mechanism by which HSV-1 counteracts antiviral responses (Figure 2D). Stable host transcripts appear to be targeted by vhs in a different way that includes binding of vhs to the cap structure via its affinity for the translation initiation factor eIF4H that causes mRNA decapping and degradation of the uncapped mRNA $5^{\prime}$ to $3^{\prime}$ [465-467]. In addition to eIF4H, vhs also interacts with other subunits of the cap structure, including the eIF4AII isoform, the eIF4B, and perhaps other components of the translation apparatus [460,468-472]. These interactions may allow vhs to access some targeted mRNAs during translation initiation and regulate their expression. Overall, vhs displays specificity since it preferentially degrades translating mRNAs and not tRNAs or rRNAs [473].

By degrading host transcripts, particularly those induced by interferons, vhs has a central role in blocking antiviral responses. It was first described that vhs may be involved in blocking immune responses when it was observed that growth of a vhs-deficient virus was rescued in mice deleted of interferon signaling receptors [135]. It was then shown that there was increased cytokine production in mice infected with a $\mathrm{U}_{\mathrm{L}} 41$-deficient mutant as compared to wild-type virus-infected mice. Additionally, $\mathrm{U}_{\mathrm{L}} 41$ mutant viruses displayed increased sensitivity to interferon- $\alpha$ and $-\beta$ compared to wild-type virus [474]. Vhs was then described to be important for blocking the activation of dendritic cells (DCs), which was found to occur in a toll-like receptor (TLR)-independent manner $[475,476]$. It was also demonstrated in mature DCs that vhs is required for HSV-1 to block phosphorylation of STAT1 and IFN $\gamma$ signaling [477]. Infection of immunocompromised mice lacking the STAT1 gene with a vhs-deficient virus did not rescue the growth of this virus and also resulted in higher induction of cytokines than in wild-type virus-infected mice, indicating that vhs has a fundamental role in promoting virus replication and that STAT1 was required to mount an appropriate non-pathological inflammatory response [478].

In addition, by degrading transcripts, vhs was found to decrease the formation of cytoplasmic stress granules (SGs) in infected cells, thus preventing activation of PKR through accumulation of dsRNAs at the site of SGs, which would otherwise lead to innate immunity activation and translation shutoff due to phosphorylation of eIF2 $\alpha$ (Figure 2D) [465,479-483]. Furthermore, vhs has been proposed, with ICP0, to block the DNA sensor IFN- $\gamma$-inducible gene IFI16 through its endoribonuclease activity, thus blocking the antiviral activities of IFI16 in multiple cell types [123].

Cumulatively, the expression of vhs has been found to benefit the virus in multiple ways, both in vitro and in vivo.

\section{Viral Kinases}

\section{1. $U_{L} 23(T K)$}

The HSV-1 thymidine kinase (TK) is a 376-aa protein, encoded by $\mathrm{U}_{\mathrm{L}} 23$. TK is responsible for phosphorylating thymidine and deoxycytidine through an ATP-dependent mechanism, though it has been described to have broad substrate specificity [484-489]. TK is also known to phosphorylate the nucleoside analogs acyclovir (ACV) and ganciclovir (GCV), which have an inhibitory effect on the viral DNA polymerase, thus blocking viral replication [485,490,491]. TK activity is conserved across other herpesviruses [488,492-498].

In vitro studies have demonstrated that TK is dispensable for virus replication in sensory neurons derived from dorsal root ganglia of rat embryos [499]. However, in vivo studies found that TK is required for virus replication in trigeminal ganglia and the brain but not in peripheral tissues of adult mice (Figure 4A) [500-503]. This is most likely because adult neurons are post-mitotic and they do not express adequate levels of cellular TK to support the growth of HSV-1 TK-null virus, unlike dividing cells. In support of this, it was shown that substitution of the viral TK with the host TK gene enabled the recombinant virus to replicate in TG [504,505]. In addition, the absence of TK activity impairs HSV-1 
reactivation from latency [426,506-509]. Particularly, it was reported that following corneal inoculation of mice, the HSV-1 TK-null virus was severely impaired for replication in TG [502,510]. However, LAT was expressed in these ganglia, suggesting that the TK-null virus can establish latency [511]. Notably, a TK-null mutant virus cannot reactivate even when latent viral loads were comparable to those that permit efficient reactivation of wildtype virus, indicating that latency establishment was not an issue [237,500,506,508,512,513].

Prolonged treatment with acyclovir and its analogs can lead to virus-acquired drug resistance because of mutations accumulating in the TK gene. Such mutant viruses are causing major problems in immunocompromised individuals in the clinic [514-516]. Considering also that the properties of HSV-1 TK have been explored in experimental therapies of intracranial tumors, it is important to clarify if HSV-1 TK-null viruses can establish lifelong infections in immunocompromised hosts. Using different HSV-1 TK mutants and different backgrounds of nude mice, it was demonstrated that all HSV-1 TK mutants can establish persistent infections in the TG and brain stem of nude mice [508]. This is consistent with the detection of ACV-resistant TK mutants in the CNS of immunocompromised patients with persistent infection [517].

While the role of TK in viral replication and latency in vivo has been the subject of a fair amount of investigation, a breadth of information has been obtained regarding the potential of TK expression for therapeutic purposes. More specifically, much work has been done on using the HSV-TK/GCV suicide gene therapy system for cancer treatment. This system works such that GCV is monophosphorylated by HSV-TK and further phosphorylated by host cell kinases. The triphosphate form of GCV is an analog of purine and it incorporates in the nascent DNA of the cancer cells, which are actively proliferating and synthesizing DNA. This causes the DNA polymerase to stall with subsequent termination of nuclear and mitochondrial DNA synthesis. As a consequence, DNA damage and cell cycle arrest is induced, leading to caspase-dependent cell death in cancer cells [518-524]. It was observed in breast cancer cells that the HSV-TK/GCV system induced p53-dependent DNA damage responses and cell cycle arrest, perturbing mitochondrial homeostasis through membrane potential dysfunction and release of cytochrome $\mathrm{c}$ into the cytoplasm in neuroblastoma cells $[519,525,526]$. Similar findings were reported in hepatocellular carcinoma cells using an adenovirus method of delivery for HSV-TK [527]. The HSV-TK/GCV system has also been seen to be effective in tumor models in mice [528-531]. This system is considered efficient because the effects of HSV-TK/GCV are also mediated through bystander effects on surrounding cells and tissues to those that uptake HSV-TK/GCV, which is thought to occur through the transfer of cytotoxic molecules between cells [532-537]. HSV-TK/GCV has since been used in several phase I/II clinical trials [538-543]. Other preclinical trials have also been published using HSV TK in other delivery systems, and frequently in combination with other antitumor therapy methods [544-550].

\section{2. $U_{S} 3$ and $U_{S} 3.5$}

The serine/threonine protein kinase $\mathrm{U}_{\mathrm{S}} 3$ of $\mathrm{HSV}-1$ has multiple significant functions, though it is non-essential in cell culture [21]. However, $U_{S} 3$ has been found to be critical for infection of both peripheral sites and the central nervous system [237,551,552]. Furthermore, $\mathrm{U}_{\mathrm{S}} 3$ has been implicated in the promotion of the viral infection in multiple ways, from blocking host responses to promoting viral replication and nuclear egress.

One function of $\mathrm{U}_{\mathrm{S}} 3$ is to promote the egress of nucleocapsids from the nucleus to the cytoplasm through primary envelopment (Figure 3) [553,554]. This is supported by the observation that in $\mathrm{U}_{\mathrm{S}} 3$-null virus-infected cells, aberrant HSV-1 capsids are trapped between the inner and the outer nuclear membrane [103]. Us3 deficiency also causes accumulation of viral proteins essential for cytoplasmic envelopment and viral infectivity, such as gK, with the capsids in the perinuclear space (PNS) [555]. To further promote nucleocapsids egress, $U_{S} 3$ has been implicated in phosphorylation of lamin $\mathrm{A} / \mathrm{C}$ that causes changes in its architecture, including changes in its localization and conformation [556-559]. $U_{S} 3$ also assists in the egress of nucleocapsids through interactions with and phosphorylation of 
$\mathrm{U}_{\mathrm{L}} 31[560]$ and $\mathrm{U}_{\mathrm{L}} 34[556,561,562]$. All three proteins are located within perinuclear virions and at the inner nuclear membrane (INM), suggesting that they could be incorporated into the virion during budding at the INM. $U_{S} 3$ also phosphorylates $U_{L} 47$ at Ser-77, promoting its nuclear localization [563]. A proposed role of nuclear $\mathrm{U}_{\mathrm{L}} 47$ is to interact with the nuclear egress factors $\mathrm{U}_{\mathrm{L}} 31, \mathrm{U}_{\mathrm{L}} 34$, and $\mathrm{U}_{\mathrm{S}} 3$ to regulate viral nuclear egress [291].

A

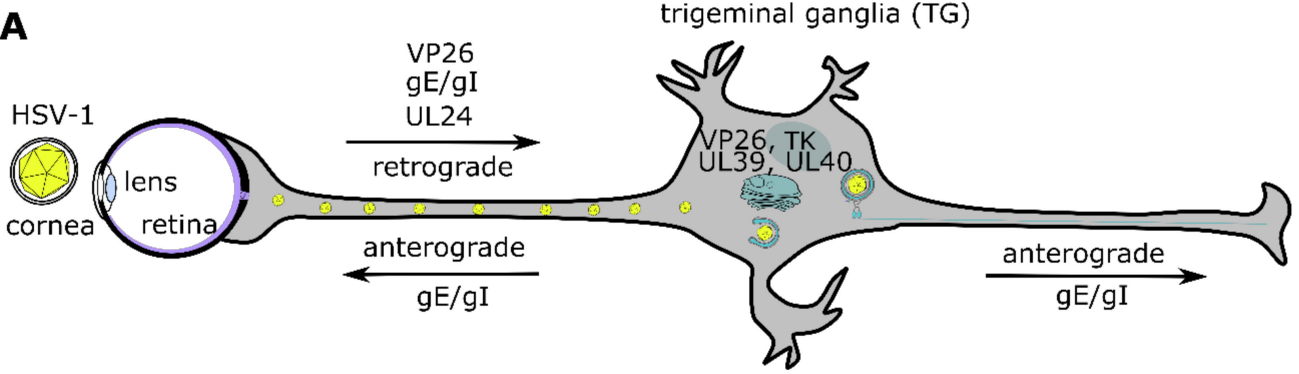

B

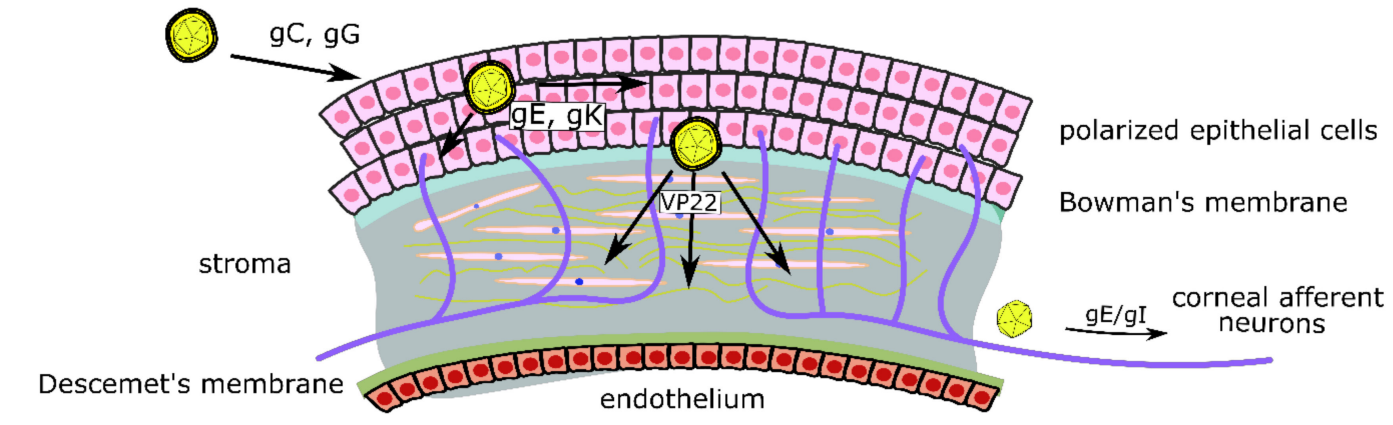

C optic nerve fibers towards cornea

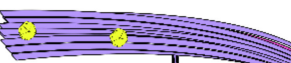

D

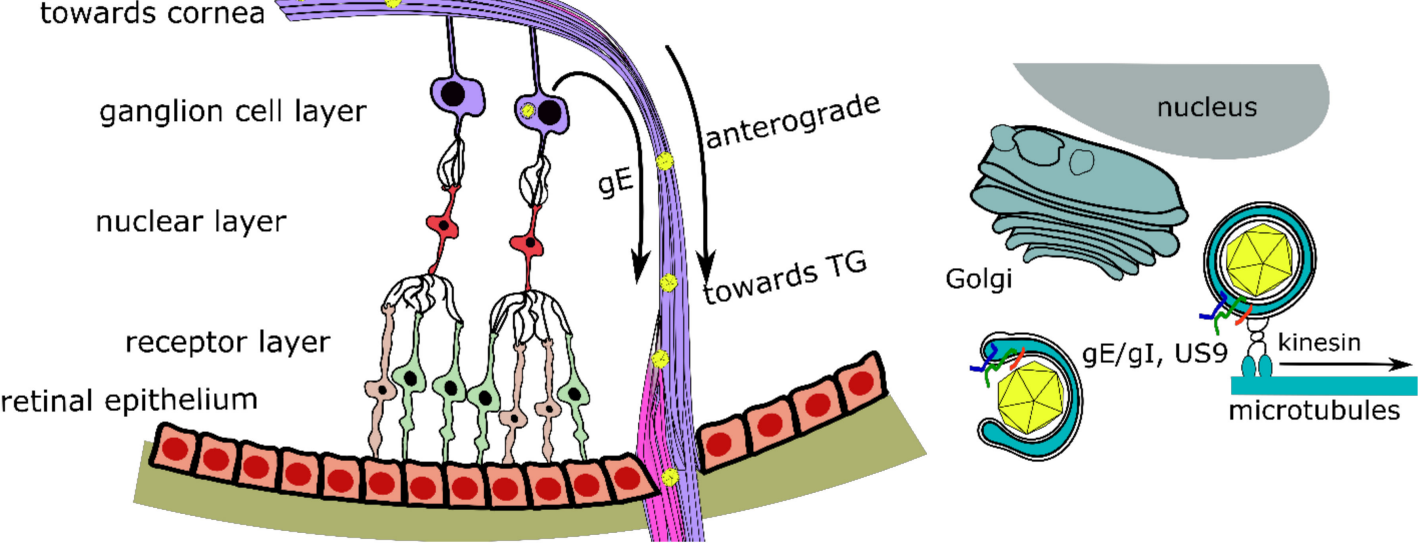

Figure 4. HSV-1 ocular infection based on data in mouse models. (A) HSV-1 can infect the eye through the cornea, where it can establish a productive infection in the corneal epithelium. The virus can then spread through the innervating sensory neurons to the trigeminal ganglia (TG), moving retrograde along axons towards the neuronal bodies. VP26 mediates migration to the TG. $\mathrm{gE} / \mathrm{gI}$ and $\mathrm{U}_{\mathrm{L}} 24$ are important for trafficking of the virion along the axons towards the neuronal bodies. $\mathrm{U}_{\mathrm{L}} 39 / \mathrm{U}_{\mathrm{L}} 40$ and $\mathrm{TK}$ are required for replication in cells that do not actively divide, such as neurons. gE/gI affect both retrograde and anterograde movement of the virion. (B) HSV-1 infection of the cornea: The virus can infect the upper layer of polarized epithelial cells, which requires $\mathrm{gC}$ and $\mathrm{gG}$. Dissemination of the ocular infection requires the function of gE and $\mathrm{gK}$, and further spread of the virus towards the underlying stromal layers of the cornea requires the function of VP22. HSV-1 can infect corneal afferent neurons and then spread towards the TG utilizing gE/gI. (C) HSV-1 can establish an infection in the retinal neuronal cells and can then move retrograde towards the cornea or anterograde towards the central nervous system (CNS). This migration requires gE, and presumably gI since they function in a complex. (D) Anterograde trafficking of virions inside neurons requires $\mathrm{gE} / \mathrm{gI}$ and $\mathrm{U}_{\mathrm{S}} 9$. Based on data in pseudorabies virus (PRV), $\mathrm{U}_{\mathrm{S}} 9$ interacts with kinesins that regulate the anterograde movement of virions along microtubules towards axonal termini. It is also possible that $\mathrm{gE} / \mathrm{gI}$ and $\mathrm{U}_{\mathrm{S}} 9$ are required for proper cytoplasmic envelopment and sorting to axons. 
Another function of $U_{S} 3$ is inhibition of apoptosis [564,565]. It has been demonstrated that expression of $U_{S} 3$ protein outside of the context of the infection mediated posttranslational modification of $\mathrm{BAD}$, a proapoptotic protein, which is no longer proapoptotic upon post-translational modifications at Ser-112 and Ser-136 by US3 [566]. Consequently, PARP cleavage, BAD cleavage, and caspase 3 activity were blocked when proteins that induce apoptosis were expressed concordantly with $U_{S} 3[470,566,567]$. Us3 may block apoptosis in multiple ways since it blocks cell death induced after infection with various HSV-1 mutants, such as $\triangle \mathrm{ICP} 4$, but it can also protect against cell death induced after thermal or osmotic shock [564,568]. In support of this, the optimal consensus sequence of $\mathrm{U}_{\mathrm{S}} 3$ peptide substrates was found to resemble the target sequence of the cellular cAMPdependent protein kinase PKA [569]. PKA is a key enzyme important in the regulation of metabolism, survival, and proliferation of eukaryotic cells, and it mediates most of the biological effects of the second messenger cAMP. The pattern of proteins phosphorylated by $\mathrm{U}_{\mathrm{S}} 3$ overlaps that of phosphoproteins targeted by PKA. Consistently, PKA could block apoptosis by stimuli that Us3 could block. Overall, $\mathrm{U}_{\mathrm{S}} 3$ can block DNA fragmentation and cell death caused by exogenous expression of pro-apoptotic factors or a variety of other stimuli.

$\mathrm{U}_{\mathrm{S}} 3$ has been implicated in promoting viral gene expression both at the level of transcription and translation and virus replication (Figure 1). $U_{\mathrm{S}} 3$ promotes viral gene transcription by preventing the deacetylation of histones, a function that also involves ICP0 $[103,570]$. The $U_{S} 3$ kinase from VZV and PRV promotes hyperphosphorylation of HDAC2 and likely HDAC1 to reduce viral genome silencing and allow efficient viral replication [570]. $\mathrm{U}_{\mathrm{S}} 3$ has also been proposed to act as the ser/thr kinase Akt, although it does not look like Akt. This Akt-like kinase function of $\mathrm{U}_{\mathrm{S}} 3$ has a role in stimulating mRNA translation through activation of mTORC1 by phosphorylating tuberous sclerosis complex 2 (TSC2) on the same sites as Akt $[571,572]$. Activated mTORC1 negatively regulates the activity of the translation repressor 4E-BP1, enabling cap-dependent translation. Additionally, $\mathrm{U}_{\mathrm{S}} 3$ phosphorylates the viral dUTPase, encoded by $\mathrm{U}_{\mathrm{L}} 50$, at Ser-187, and that causes an increase of its activity over host dUTPases, thereby promoting HSV-1 replication [411].

Finally, $\mathrm{U}_{\mathrm{S}} 3$ is implicated in virus defense against the host. One example is the requirement of $\mathrm{U}_{\mathrm{S}} 3$ for inactivation of $\mathrm{CD} 8^{+}$cytotoxic $\mathrm{T}$ lymphocytes, thus preventing cytokine production [573]. $\mathrm{U}_{\mathrm{S}} 3$ also blocks TLR2 signaling early during infection by preventing TRAF6 polyubiquitination [574]. Additionally, $U_{\mathrm{S}} 3$ hyper-phosphorylates IRF3 at Ser175, which inhibits IFN- $\beta$ production [575]. Furthermore, the Bcl-2-associated transcription factor 1 (Bclaf1) was recently shown to be degraded in a $\mathrm{U}_{\mathrm{S}} 3$-dependent manner during HSV-1 infection [576]. This degradation prevented IFN- $\alpha$-mediated interferon-stimulated genes expression [576]. $U_{S} 3$ also phosphorylates both ULK1 and Beclin-1, thus blocking autophagy activation during HSV-1 infection in a manner independent of ICP34.5 [577]. In a different approach to promoting the viral infection, $\mathrm{U}_{\mathrm{S}} 3$ has also been found to affect the subcellular localization of certain viral proteins by affecting their phosphorylation status. Particularly, $\mathrm{U}_{\mathrm{S}} 3$ causes a decrease in the amount of $\mathrm{gB}$ found on the cell surface by phosphorylating its cytoplasmic tail at Thr-887 and perhaps enhancing its endocytosis [578]. This phosphorylation of $\mathrm{gB}$ has been implicated in virus pathogenesis since mutation of Thr-887 significantly impaired viral replication in the mouse cornea and the development of herpes stromal keratitis and periocular skin disease [578]. $U_{S} 3$ has also been found, with $\mathrm{gB}$, to be involved in downregulation of the major histocompatibility complex class I-like antigen-presenting molecule, CD1d, through prevention of recycling of CD1d to the cell surface [579].

A shorter version of Us3 named Us3.5 was discussed earlier together with ICP22.

Overall, $\mathrm{U}_{\mathrm{S}} 3$ plays many important roles in the viral life cycle and in viral infectivity.

\section{3. $U_{L} 13$}

In addition to the $\mathrm{U}_{\mathrm{S}} 3$ kinase, HSV-1 encodes a second serine/threonine protein kinase $U_{L} 13$, which functions in the cell nuclei and is present in the virion as a tegument 
protein associated with the capsid [580-584]. $\mathrm{U}_{\mathrm{L}} 13$ is conserved across members of alpha-, beta-, and gamma-herpesviruses [380,585-589]. $\mathrm{U}_{\mathrm{L}} 13$ is non-essential in cell culture, but it seems to have a role in counteracting antiviral responses and is important for optimal viral replication in cell culture [590-592]. In a mouse model, a $\mathrm{U}_{\mathrm{L}} 13$-deleted virus was sensitive to type I IFN, suggesting an important role for $\mathrm{U}_{\mathrm{L}} 13$ in blocking host responses to infection [593].

$\mathrm{U}_{\mathrm{L}} 13$ phosphorylates multiple viral proteins, including itself, the immediate-early viral protein ICP22 and $\mathrm{U}_{\mathrm{S}} 1.5, \mathrm{ICP} 0$, and numerous tegument and envelope proteins (Figure 1) $[190,242,253,254,592,594,595]$. It seems that phosphorylation of ICP0 by $\mathrm{U}_{\mathrm{L}} 13$ is important for stabilizing ICP0 protein during infection [596]. This is supported by the fact that ICP0 is degraded both early and late in cells infected with a mutant lacking the $\mathrm{U}_{\mathrm{L}} 13$ protein kinase. Furthermore, it was found that ICP0 encoded by wild-type virus or the $\mathrm{U}_{\mathrm{L}} 13-$ null mutant is stable in cells transfected with a plasmid encoding $\mathrm{U}_{\mathrm{L}} 13$ before infection [596]. Phosphorylation of $\mathrm{U}_{\mathrm{L}} 46$ by $\mathrm{U}_{\mathrm{L}} 13$ leads to Akt activation to promote cell survival [597]. $\mathrm{U}_{\mathrm{L}} 13$ also phosphorylates VP22 at casein kinase II consensus sites, but it was found that $\mathrm{U}_{\mathrm{L}} 13$ modulates cellular localization of VP22 in a phosphorylationindependent manner [590,595,598]. The significance of phosphorylation of VP22 by $\mathrm{U}_{\mathrm{L}} 13$ has not been investigated. $\mathrm{U}_{\mathrm{L}} 13$ phosphorylates the viral Fc receptor $\mathrm{gE} / \mathrm{gI}$, though it seems that a host kinase may also phosphorylate $\mathrm{gE} / \mathrm{gI}$ [599]. Phosphorylation of $\mathrm{gE}$ by $U_{L} 13$ is thought to facilitate packaging of $U_{L} 13$ into the virion [599]. However, this phosphorylation could also impact $\mathrm{gE}$ trafficking or other functions. $\mathrm{U}_{\mathrm{L}} 13$ expression was found to be downregulated by the $U_{S} 11$ RNA-binding protein. $U_{S} 11$ was found to bind to the RNA sequence, designated as 12/14, which is present in the coterminal HSV-1 mRNAs $\mathrm{U}_{\mathrm{L}} 12, \mathrm{U}_{\mathrm{L}} 13$, and $\mathrm{U}_{\mathrm{L}} 14$. This binding led to reduced $\mathrm{U}_{\mathrm{L}} 13$ kinase activity due to reduced mRNA levels [600]. While the exact mechanism of downregulation of $U_{L} 13$ transcripts by $\mathrm{U}_{\mathrm{S}} 11$ is unknown, it is thought that this occurs through nucleocytoplasmic export of the transcript [600]. The kinase activity of $U_{L} 13$ and $U_{S} 3$ was found to be important for the viral glycoproteins $\mathrm{gC}$ and $\mathrm{gD}$ to be modified and expressed late during infection, as loss of both $\mathrm{U}_{\mathrm{L}} 13$ and $\mathrm{U}_{\mathrm{S}} 3$ diminished virion release, showing a role for $\mathrm{U}_{\mathrm{L}} 13$ in assembly and egress of the virion [601]. Thus, $\mathrm{U}_{\mathrm{L}} 13$ modifies multiple viral proteins both to promote assembly and release of the virus, and to affect the stability and localization of viral proteins.

$\mathrm{U}_{\mathrm{L}} 13$ plays roles in modulating host responses to infection. For example, interferon stimulation and production of cytokines are modulated by $U_{L} 13$ during infection. $U_{L} 13$ has been found to be important for the induction of a set of suppression of cytokine signaling (SOCS) genes late during infection, which is important for blocking the interferon response during HSV-1 infection of cells [602-605]. Recently, $\mathrm{U}_{\mathrm{L}} 13$ was found with the other viral kinase $U_{S} 3$ to be important for regulating phosphorylation of protein kinase $R$ (a nucleic acid sensor) during infection [606]. $\mathrm{U}_{\mathrm{L}} 13$ was also found to hyperphosphorylate an important host factor for the elongation of peptide chains during mRNA translation, eF-1 $\delta$ during HSV-1 infection, supporting viral protein synthesis, and also preventing apoptosis [607-609]. $\mathrm{U}_{\mathrm{L}} 13$ was found to phosphorylate the cellular casein kinase II $\beta$ subunit (CKII $\beta$ ), though the significance of this phosphorylation has not been explored [610]. However, it was found that $\mathrm{U}_{\mathrm{L}} 13$ is able to phosphorylate proteins at similar residues as the cellular cdc2 cyclin kinase [610]. It was also determined that $\mathrm{U}_{\mathrm{L}} 13$, with ICP22, is responsible for activating cdc2 during infection, which is required for optimal expression of viral late genes $[259,260]$. $\mathrm{U}_{\mathrm{L}} 13$ was also found with ICP22 to phosphorylate the RNA polymerase II (RNAP II), supporting virus late gene expression [267]. Overall, phosphorylation of host proteins by $\mathrm{U}_{\mathrm{L}} 13$ supports viral infection by supporting virus late gene expression, blocking innate immune responses, supporting viral protein synthesis, and activating cellular proteins for the benefit of the virus. 


\section{Virion Morphogenesis, Egress, Cell-to-Cell Spread, and Host Evasion 6.1. $U_{L} 3$ and $U_{L} 4$}

The HSV-1 $\mathrm{U}_{\mathrm{L}} 3$ and $\mathrm{U}_{\mathrm{L}} 4$ are late proteins that have only minorly been studied, though they have been determined to be nonessential in cell culture and $U_{L} 4$ was also found to be nonessential in mouse models of HSV-1 infection for latency, reactivation, and pathogenesis $[421,611,612]$. The $\mathrm{U}_{\mathrm{L}} 3$ phosphoprotein and the $\mathrm{U}_{\mathrm{L}} 4$ protein have homologs in other members of the Herpesviridae family [84,153,368-370,613-620]. $\mathrm{U}_{\mathrm{L}} 3$ appears to localize perinuclearly early during infection and in nuclear puncta at late times post infection $[613,621,622] . \mathrm{U}_{\mathrm{L}} 3$ and $\mathrm{U}_{\mathrm{L}} 4$ have also been found in the nuclei of infected cells and found to co-localize with ICP22 in nuclear bodies, which may involve recruitment by ICP22 [271,623-625].

\section{2. $U_{L} 7$ and $U_{L} 51$}

HSV-1 $\mathrm{pU}_{\mathrm{L}} 7$ is a 296-aa tegument protein [626] that lacks a putative transmembrane sequence or motifs that could facilitate its membrane anchor. Therefore, its association with membranes is mediated through the interaction with a membrane protein, i.e., $\mathrm{U}_{\mathrm{L}} 51 . \mathrm{U}_{\mathrm{L}} 7$ forms a complex with $U_{L} 51$, and this is required for the recruitment of $U_{L} 7$ to cytoplasmic membranes and into the virion tegument (Figure 3C) [627]. $\mathrm{pU}_{\mathrm{L}} 7$ and $\mathrm{pU}_{\mathrm{L}} 51$ form a stable and direct protein-to-protein interaction [628], and they function as a complex in infected cells. Both are important for HSV-1 assembly and plaque formation. Their individual ablation results in similarly lower yields with a double $\mathrm{U}_{\mathrm{L}} 7 / \mathrm{U}_{\mathrm{L}} 51$ knockdown, suggesting that $\mathrm{U}_{\mathrm{L}} 7$ and $\mathrm{U}_{\mathrm{L}} 51$ work in the same pathway [628], which is likely related to cytoplasmic envelopment of HSV-1 virions since many unenveloped capsids next to membranes can be seen in cells infected with $\mathrm{U}_{\mathrm{L}} 7 / \mathrm{U}_{\mathrm{L}} 51$ mutants [628].

$\mathrm{U}_{\mathrm{L}} 7$ ablation can affect HSV-1 infection at earlier times, too. $\mathrm{U}_{\mathrm{L}} 7$ absence results in viruses with lower yields in vitro and lower pathogenic effects in vivo [629]. Mice infected with a $\mathrm{U}_{\mathrm{L}} 7$ mutant HSV-1 exhibit longer survival than those infected with WT HSV-1. A decrease in LAT mRNA expression was observed in the CNS and trigeminal ganglia of mice infected with a $\mathrm{U}_{\mathrm{L}} 7$ mutant $\mathrm{HSV}-1$, suggesting decreased viral gene transcription in the absence of $\mathrm{U}_{\mathrm{L}} 7$. This is supported by data that show that $\mathrm{U}_{\mathrm{L}} 7$ may participate in the complex that is involved in the transcription of ICP4 [629]. It is not clear if $\mathrm{U}_{\mathrm{L}} 7$ is directly involved in the interaction between the promoter of ICP4 and the transcriptional complex or is involved perhaps in the chromatin remodeling process that enables transcription of ICP4. $\mathrm{U}_{\mathrm{L}} 7$ can be detected through cell fractionation and fluorescence microscopy in the nucleus [630], although it is primarily seen in the cytoplasm. It is not clear if its function in the nucleus is derived from $\mathrm{U}_{\mathrm{L}} 7$ delivered as part of the incoming virions [627] or from nascently expressed $\mathrm{U}_{\mathrm{L}} 7$.

$\mathrm{U}_{\mathrm{L}} 7$ and $\mathrm{U}_{\mathrm{L}} 51$ can also affect cell-to-cell spread of HSV-1. pUL51 interacts with $\mathrm{p} \mathrm{U}_{\mathrm{L}} 7$ and $\mathrm{gE} / \mathrm{gI}$ in infected cells, and deletion of part of $\mathrm{U}_{\mathrm{L}} 51$ or deletion of $\mathrm{U}_{\mathrm{L}} 7$ results in failure of $\mathrm{gE}$ to concentrate at junctional surfaces of Vero cells (Figure 3). This suggests a role for a $\mathrm{U}_{\mathrm{L}} 51 / \mathrm{U}_{\mathrm{L}} 7$ complex in cell-to-cell spread of HSV-1; however, this may depend on the cell line. A $\mathrm{pU}_{\mathrm{L}} 7 / \mathrm{pU}_{\mathrm{L}} 51 / \mathrm{gE} / \mathrm{gI}$ method for cell-to-cell spread can occur in polarized epithelial cells, such as $\mathrm{HaCaT}$, but different cell-to-cell spread mechanisms may be utilized in non-polarized cells, such as Vero [631]. Additionally, the $\mathrm{U}_{\mathrm{L}} 7 / \mathrm{U}_{\mathrm{L}} 51$ complex can affect cell-to-cell spread by localizing to focal adhesions in infected cells. Focal adhesions are contact sites between the cytoplasm and the extracellular matrix. They are dynamic and respond to extracellular stimuli and play a role in cell attachment and movement. Ablation of the $\mathrm{U}_{\mathrm{L}} 7 / \mathrm{U}_{\mathrm{L}} 51$ complex results in destabilization of focal adhesions and diminished cell integrity [628]. $U_{L} 7$ and $U_{L} 51$ seem to mediate the stability of focal adhesions during infection, perhaps to maintain the proximity of infected and non-infected cells so that cell-to-cell spread can be promoted. This still remains to be demonstrated.

An additional function of $\mathrm{U}_{\mathrm{L}} 7$ may be exhibited on the mitochondria. $\mathrm{U}_{\mathrm{L}} 7$ has been identified as a partner of the adenine nucleotide translocase (ANT2) through a mass spectrometry approach combined with affinity purification. ANT2 localizes in the inner 
mitochondrial membrane and is a member of the permeability transposition pore complex. In physiological conditions, it exchanges ATP and ADP on the inner mitochondrial membrane and is essential for maintaining the cell metabolism exchange of cytosolic ADP for mitochondrial ATP. HSV-1 infection can affect mitochondrial function, and $\mathrm{U}_{\mathrm{L}} 7$ may be one of the proteins that are important for this function [632].

$\mathrm{U}_{\mathrm{L}} 51$ is a late gene that is expressed as three phosphoproteins with sizes of 27,29 , and $30 \mathrm{kDa}$. It can be detected in extracellular HSV-1 virions [633], is phosphorylated on five sites [634], and phosphorylation on the Ser-184 site has been described as important for HSV1 replication in vitro and pathogenicity in vivo after ocular infection of mice [634]. $U_{L} 51$ localizes in the cytoplasm [633], mostly in the perinuclear area, but part of it also localizes to the Golgi. Golgi localization requires the N-terminus of $U_{L} 51$, which is palmitoylated to mediate sorting to Golgi membranes. $\mathrm{U}_{\mathrm{L}} 51$ is packaged in virions, on the inside of the viral envelope [633]. $U_{\mathrm{L}} 51$ internalization into vesicles and virions may occur during cytoplasmic envelopment in infected cells [633]. Infections with a $U_{\mathrm{L}} 51$-null HSV-1 yields smaller plaques and a growth of 2 logs lower than a WT HSV-1. $U_{\mathrm{L}} 51$-null infections exhibit enveloped virions at the perinuclear space, as opposed to enveloped virions at membranes at the TGN during WT HSV-1 infections [635]. The membranes that encapsulate those $\mathrm{U}_{\mathrm{L}}$ 51-null virions resemble nuclear membranes through electron microscopy. Such membranes are tightly wrapped around nucleocapsids and do not appear as fuzzy as the membrane of the extracellular virions. Additionally, nucleocapsids are found intranuclearly adjacent to the inner nuclear membrane (INM) with membranes with the same appearance, further supporting that these perinuclear enveloped virions are enveloped with membranes derived from the nuclear cisternae. This suggests that $U_{\mathrm{L}} 51$ acts at a post-inner nuclear membrane envelopment step, possibly during the outer nuclear membrane de-envelopment process (Figure 3B).

As mentioned above, $\mathrm{U}_{\mathrm{L}} 51$ has a role in cell-to-cell spread that is dependent on cell type. $\mathrm{U}_{\mathrm{L}} 51$ colocalizes with $\mathrm{gE}$ in infected cells and it can be immunoprecipitated together with $\mathrm{gE}$, in addition to affecting gE localization to cell junctions [636]. It is possible that $\mathrm{U}_{\mathrm{L}} 51$ functions as a trafficking mediator while is present on the cytoplasmic side of Golgi membranes.

$\mathrm{U}_{\mathrm{L}} 51$ recruits $\mathrm{U}_{\mathrm{L}} 7$ into the nascent virion tegument (Figure 3) [627]. Their colocalization is incomplete though, suggesting that they have other independent functions [627].

$\mathrm{U}_{\mathrm{L}} 51$ also interacts with $\mathrm{U}_{\mathrm{L}} 14$ in infected cells, as shown by affinity purification [637]. Three amino acids on $\mathrm{U}_{\mathrm{L}} 51$ are required for this interaction, and their mutation results in decreased viral replication and accumulation of unenveloped and partially enveloped capsids in the cytoplasm. The localization of both $\mathrm{U}_{\mathrm{L}} 51$ and $\mathrm{U}_{\mathrm{L}} 14$ depends on their reciprocal interaction. These data suggest the $\mathrm{U}_{\mathrm{L}} 51-\mathrm{U}_{\mathrm{L}} 14$ complex regulates cytoplasmic envelopment of HSV-1 [637].

\section{3. $U_{L} 10$ and $U_{L} 49.5$ ( $g M$ and $g N$ )}

Glycoprotein $\mathrm{M}(\mathrm{gM})$ is an integral viral envelope membrane protein that spans the membrane eight times [638]. Its deletion results in only a small decrease in viral yields in cell culture [611,639], thus it is defined as non-essential. Even though a gM-null virus can establish a latent infection in mice, it is impaired for growth within the nervous system versus the wild-type virus [638].

gM localizes within the leaflets of the nuclear membrane, at the Golgi and the TGN, and the envelopes of cytoplasmic and extracellular virus particles [640]. Infection with a $\mathrm{U}_{\mathrm{S}} 3$-null HSV-1 results in punctate extensions and invaginations of the nuclear membrane, on which gM localizes [640]. This suggests that gM becomes incorporated into the virion envelope upon budding through the nuclear membrane. Transfection of gM leads to its localization to the TGN and plasma membrane (PM) [641,642]; however, this pattern changes during infection. When gM is expressed, it is recruited to nuclear membranes and then to perinuclear virions once they are formed. This occurs before HSV-1 induces reorganization of the TGN and before gM localization to the TGN. Consistent with these observations, 
confirmed partners of gM, such as $\mathrm{gH} / \mathrm{gL}$, gN, VP22, $\mathrm{U}_{\mathrm{L}} 31$, and $\mathrm{U}_{\mathrm{L}} 34$ [643-646], do not colocalize with gM early during infection. Therefore, it has been proposed that the function of gM early during infection in the nuclear membranes is separate from its function during viral egress [641]. Characterization of gM domains showed that its trafficking to the TGN requires its transmembrane domains, while its $\mathrm{C}$-terminal trafficking motifs are dispensable. The requirement of the transmembrane domains suggests that $\mathrm{gM}$ may associate with other transmembrane proteins for trafficking (Figure 3C) [647], but this remains to be shown.

The role of gM in the TGN is related to the trafficking of host and viral proteins during infection. For example, co-expression of $\mathrm{gB}, \mathrm{gD}, \mathrm{gH}$, and $\mathrm{gL}$ can trigger fusion of cell membranes of transfected cells. Such fusion can be inhibited by the additional co-expression of $\mathrm{gM}$ [643]. In this context, $\mathrm{gD}$ and $\mathrm{gH} / \mathrm{gL}$ can be seen to relocalize from the plasma membrane to the TGN, suggesting that inhibition of fusion is triggered through the removal of the fusion glycoproteins from the surface [643]. These data suggest that gM is involved in either retaining viral glycoproteins at the TGN or causing their translocalization from the plasma membrane to the TGN, supporting virion maturation at the TGN [648]. Further supporting data show that an absence of gM results in reduced $\mathrm{gH} / \mathrm{gL}$ internalization from the PM of infected cells, and reduced incorporation in produced virions [649].

$\mathrm{gM}$ can interact with $\mathrm{gN}$, resulting in altered intracellular targeting of both proteins. Co-immunoprecipitations in transfected or infected cells indicate that gM and $\mathrm{gN}$ form a complex [642], and gN overexpression seems to mediate the formation of syncytia in infected cells, which are inhibited normally by gM $[642,643,650]$. Syncytia occurs when cell membranes fuse, forming large multinucleated cells. This suggests a strict regulation of fusion that can be deregulated by altered $\mathrm{gN}$ expression possibly through altering the localization of $\mathrm{gD}$ and $\mathrm{gH} / \mathrm{gL}$ from the plasma membrane to the TGN that is triggered by $\mathrm{gM}$ [643]. $\mathrm{gN}$ is an ER-resident protein that in the presence of $\mathrm{gM}$ is translocated to the TGN. gM and gN are covalently linked between two cysteines, and exit of $\mathrm{gN}$ from the ER requires the $\mathrm{N}$-terminus of $\mathrm{gM}$ but not the $\mathrm{C}$-terminus. $\mathrm{gN}$ is non-essential and its deletion does not seem to affect viral growth [644].

While gM can inhibit syncytium formation in transfected cells [643] and reduces the surface expression of proteins involved in fusion, only gN and $\mathrm{U}_{\mathrm{L}} 46$ have been identified as partners of HSV-1 gM. Proteomics studies with an emphasis on host proteins identified the host extended synaptotagmin 1 (E-Syt1) as a gM partner [651]. E-Syt proteins promote the close apposition of the ER and the plasma membrane (PM), and the transfer of lipids between the ER and the PM. Functions of several synaptotagmins remain to be determined, but they seem to engage and regulate SNARE proteins (the core cellular fusion machinery) and act as $\mathrm{Ca}^{2+}$ sensors.

It was found that during HSV-1 infection, knocking down E-Syt1 triggered the release of the virus into the extracellular space, at the expense of cell-associated infectious particles. Conversely, overexpressing E-Syt1 led to reduced levels of mature virions in the medium, hinting at a negative regulation. E-Syt1 did not act alone but in combination with the related E-Syt3, which exhibited a similar phenotype. Most interestingly, these E-Syt proteins impacted viral entry, as well as cell-cell fusion (syncytia) and viral plaque size (cell-tocell spread), suggesting they acted on the viral fusion machinery [651]. One possible mechanism of action might involve deregulation of $\mathrm{Ca}^{2+}$ signaling that occurs during HSV-1 infection [652,653], and which could affect E-Syt $\mathrm{Ca}^{2+}$-dependent function [651].

A BioID proteomics approach identified multiple gM partners, with $35 \%$ of those being involved in protein transport. XPO6, an exportin, is required for gM to be released from the nucleus to the TGN [654].

Another interesting way that gM is involved in affecting host trafficking is through modulation of tetherin (Figure 3). Tetherin is an effective cellular factor against a variety of enveloped viruses. Its antiviral activity stems from its ability to form a tether between a host membrane and a budding viral envelope, inhibiting the release of budding virions [655]. Tetherin can also target HSV-1, as the overexpression of tetherin led to accumulation of HSV-1 particles to the cell surface, suggesting inhibition of HSV-1 release [656]. HSV-1 
counteracts tetherin function through gM, which interacts with tetherin and removes it from the plasma membrane, thus preventing virion tethering to the plasma membrane. This antagonistic effect might be due to gM preventing tetherin reaching the cell surface or relocalizing tetherin away from the plasma membrane [656], perhaps in a similar manner with $\mathrm{gD}$ and $\mathrm{gH} / \mathrm{Gl}[643]$.

\section{4. $U_{L} 11$}

$\mathrm{U}_{\mathrm{L}} 11$ is an early expressed 96-aa myristylated and palmitoylated tegument protein [657-659] that is not required for HSV-1 replication in cell culture. A $U_{\mathrm{L}} 11$-null virus exhibits smaller plaques and displays about one log decrease in progeny virus production [660]. Additionally, the palmitoylation and myristylation of $\mathrm{U}_{\mathrm{L}} 11$ is not required for viral growth, since a non-myristylated $\mathrm{U}_{\mathrm{L}} 11$ mutant HSV-1 can rescue the growth of a $\mathrm{U}_{\mathrm{L}}$ 11-null HSV-1 [661]. The palmitoylation of $\mathrm{U}_{\mathrm{L}} 11$ is required though for association of $\mathrm{U}_{\mathrm{L}} 11$ with the cytoplasmic faces of Golgi membranes of infected cells $[658,660]$.

$\mathrm{U}_{\mathrm{L}} 11$ can interact with several viral proteins, including $\mathrm{U}_{\mathrm{L}} 16$, as observed through immunoprecipitation, mass spectrometry, and yeast-two-hybrid assays $[155,662]$. There are dileucine and acidic cluster motifs on $\mathrm{U}_{\mathrm{L}} 11$ that are required for the $\mathrm{U}_{\mathrm{L}} 11-\mathrm{U}_{\mathrm{L}} 16$ interaction [659], as well as the free cysteines of $\mathrm{U}_{\mathrm{L}} 16$ [663].

It is not clear what the mechanism of the packaging of $\mathrm{U}_{\mathrm{L}} 11$ in the tegument of nascent virions is [659]. Tandem affinity purification (TAP) supports that $\mathrm{U}_{\mathrm{L}} 11$ interacts specifically with the cytoplasmic domain of $\mathrm{gD}$ and $\mathrm{gE}$ [664]. In the absence of the cytoplasmic tail of $\mathrm{gE}$, virion packaging of $\mathrm{U}_{\mathrm{L}} 11$ was reduced by $80 \%$ [665]. Similarly, gE packaging is reduced $85 \%$ in the absence of $U_{L} 11$, as $g E$ packaging requires the $U_{L} 11$ acidic cluster [665]. These data highlight the importance of $\mathrm{U}_{\mathrm{L}} 11$ in recruiting glycoprotein-enriched membranes for cytoplasmic envelopment of the virus and could have implications for $\mathrm{gE} / \mathrm{gI}$-mediated cell-to-cell spread of HSV-1 (Figure 3C,D) [665]. Interestingly, deletion of the gD cytoplasmic domain still allows partial binding of $\mathrm{U}_{\mathrm{L}} 11$ to the ectodomain of $\mathrm{gD}$, suggesting that $\mathrm{U}_{\mathrm{L}} 11$ can be highly adherent ("sticky") [664]. This would support that adherent tegument proteins can support extensive protein-protein interactions, which would mediate the bridging of the viral capsid to the envelope. $\mathrm{U}_{\mathrm{L}}$ 11-null virus infections result in accumulation of unenveloped capsids in the cytoplasm surrounded by electron-dense material (most likely other tegument proteins) [666].

The "stickiness" of $U_{\mathrm{L}} 11$ can be explained by its description as an intrinsically disordered protein (IDP). IDPs contain amino acids and elements that cause them to exhibit hallmarks of a disordered structure, such as slower electrophoretic mobility than expected based on length, reduced size exclusion chromatography mobility due to reduced protein compaction, and low proportion of hydrophobic amino acids with a high proportion of charged and hydrophilic amino acids. The result is a protein that cannot fold spontaneously into a stable conformation and fluctuates rapidly through a range of conformations. Such proteins frequently interact and function within protein-protein interaction networks [667]. As a result, $\mathrm{U}_{\mathrm{L}} 11$ can undergo phase separation in vitro and form biomolecular membraneless condensates. Such condensates can contain one or many different kinds of proteins. Specific conditions may mediate the formation of condensates by $U_{L} 11$ in cells, such as binding to $\mathrm{gE}, \mathrm{U}_{\mathrm{L}} 16$, or clustering within lipid rafts. Other HSV-1 tegument proteins also have IDP regions, indicating that phase separation may be used for tegument packaging during HSV-1 cytoplasmic envelopment [668].

If one deletes the dileucine and acidic cluster $(A C)$ motifs of $U_{L} 11$, then $U_{L} 11$ increases its association with detergent-resistant membranes (DRMs), which are enriched in cholesterol and sphingolipids. One possibility is that the deletion of dileucine motifs and ACs results in palmitoylation and myristylation to recruit $\mathrm{U}_{\mathrm{L}} 11$ to DRMs [669].

\section{5. $U_{L} 16$}

$\mathrm{U}_{\mathrm{L}} 16$ is an unusual gene because it is contained within the intron of the $U_{L} 15$ gene and is transcribed antisense to the $\mathrm{U}_{\mathrm{L}} 15$ gene [611]. $\mathrm{U}_{\mathrm{L}} 16$ has a bewildering number of 
interactions with $\mathrm{gE}, \mathrm{U}_{\mathrm{L}} 11, \mathrm{U}_{\mathrm{L}}$ 21, VP22 (Figure 3) [203], gD, and mitochondria [670]. These interactions are probably regulated temporally but also structurally by different domains of $\mathrm{U}_{\mathrm{L}} 16$.

$\mathrm{U}_{\mathrm{L}} 16$ resides on cytoplasmic capsids [671] and participates in a bridging interaction with membrane-bound $\mathrm{U}_{\mathrm{L}} 11$ [203,662]. This suggests a role for $\mathrm{U}_{\mathrm{L}} 16$ in HSV-1 cytoplasmic envelopment, which became clear in electron microscopy studies of cells infected with $\mathrm{U}_{\mathrm{L}}$ 16-null HSV-1 [203]. No defects in the transport of capsids to cytoplasmic membranes were observed, but the wrapping of capsids with membranes was delayed. Moreover, clusters of cytoplasmic capsids were observed but only near membranes where they were wrapped, resulting in multiple capsids within a single envelope. Post-envelopment egress does not require $U_{L} 16$, and viruses released in the supernatant were not affected by $U_{L} 16$ ablation [203]. Additionally, less gE and less gD were packaged in $\mathrm{U}_{\mathrm{L}}$ 16-null viruses, which is expected since $U_{L} 16$ interacts with both [672]. These data support a role for $\mathrm{U}_{\mathrm{L}} 16$ in cytoplasmic envelopment. Cell-to-cell spread is also blocked during $\mathrm{U}_{\mathrm{L}} 16$-null virus infection, which may be due to mislocalization of $g E$, since $g E$ and $U_{L} 16$ form a complex [672].

The structural modulation of $\mathrm{U}_{\mathrm{L}} 16$ interactions became clear in the studies of the $\mathrm{U}_{\mathrm{L}} 16 / \mathrm{U}_{\mathrm{L}} 11 / \mathrm{U}_{\mathrm{L}} 21 / \mathrm{gE}$ complex. $\mathrm{U}_{\mathrm{L}} 16$ directly interacts with $\mathrm{U}_{\mathrm{L}} 11$, which resides on the cytoplasmic side of the TGN. This interaction requires most of the $\mathrm{U}_{\mathrm{L}} 16$ sequence except the first 40 aa, and does so in a manner that requires free cysteines on $U_{L} 16$ [663]. Covalent modification of the $\mathrm{U}_{\mathrm{L}} 16$ free cysteines with $\mathrm{N}$-ethylmaleimide blocks binding to $\mathrm{U}_{\mathrm{L}} 11$ but not to $U_{L} 21$ [673], suggesting a binding site on $U_{L} 16$ for $U_{L} 11$ and another for $U_{L} 21$ (Figure 3C).

Interestingly, $\mathrm{U}_{\mathrm{L}} 16$ is released from capsids upon binding of HSV-1 virions to cells, but it is not clear if it maintains its interaction with $\mathrm{U}_{\mathrm{L}} 11$ [674]. For $\mathrm{U}_{\mathrm{L}} 16$ to receive a signal from outside the virion, it must interface in some manner with glycoproteins on the surface of the virion. One possibility is through $\mathrm{gE}$, with which $\mathrm{U}_{\mathrm{L}} 16$ interacts, as discussed above. The N-terminus of $\mathrm{U}_{\mathrm{L}} 16$ can bind $\mathrm{gE}$ but the full length cannot, indicating a possible regulatory effect of the $U_{L} 16$ C-terminus on the $U_{L} 16-g E$ interaction [675]. This interaction may have multiple effects, including effects on cytoplasmic envelopment described above. Additionally, since gE is involved in cell-to-cell spread, it may be involved in rearrangements that occur upon binding of virions to cell entry receptors, which results in release of $U_{\mathrm{L}} 16$ from the viral capsid [663].

The inconsistency between the N-terminus and the full-length binding of $U_{L} 16$ with $\mathrm{gE}$ became clearer when it was shown that $\mathrm{U}_{\mathrm{L}} 21$ binding to $\mathrm{U}_{\mathrm{L}} 16$ reveals the $\mathrm{U}_{\mathrm{L}}$ 11-binding free cysteine-requiring site of $\mathrm{U}_{\mathrm{L}} 16$. Then, $\mathrm{U}_{\mathrm{L}} 11$ binds to $\mathrm{U}_{\mathrm{L}} 16$ and this event activates the $U_{\mathrm{L}} 16-\mathrm{gE}$ interaction. Importantly, the function of $\mathrm{gE}$ is dependent on $\mathrm{U}_{\mathrm{L}} 11, \mathrm{U}_{\mathrm{L}} 16$, and $U_{L} 21$, as evidenced by infections with HSV-1 gBsyn mutants that lack $U_{L} 11, U_{L} 16$, or $\mathrm{U}_{\mathrm{L}} 21$. The syncytial phenotype of gBsyn HSV-1 infections requires functional $\mathrm{gE}$, and syncytia cannot form in the absence of $U_{L} 11, U_{L} 16$, and $U_{L} 21$. Cell-to-cell spread involves the localization of $g E$ to junctions at the cell surface, and in the absence of $U_{L} 11, U_{L} 16$, or $\mathrm{U}_{\mathrm{L}} 21$, gE cannot localize there. Collectively, these data suggest that these proteins work as a complex during HSV-1 infection [676].

One interesting aspect is the species-specific requirement for the $U_{L} 16$ protein when comparing HSV-1 and HSV-2 [677]. Depletion of $\mathrm{U}_{\mathrm{L}} 16$ in HSV-2 results in 50- to 100-fold lower viral yields, with defects in both nuclear egress and cytoplasmic envelopment. In contrast, depletion of $\mathrm{U}_{\mathrm{L}} 16$ in HSV-1 results in a 10-fold replication deficiency and defects in cytoplasmic envelopment of viral capsids. HSV-1 $\mathrm{U}_{\mathrm{L}} 16$ can promote the nuclear egress of HSV-2 $\mathrm{U}_{\mathrm{L}}$ 16-null mutants, suggesting that HSV-2 lacks an activity that can promote nuclear egress in the absence of $\mathrm{U}_{\mathrm{L}} 16$, as opposed to HSV-1 [677].

$A U_{L} 16-n u l l$ virus is greatly diminished in its ability to package $g D$. $U_{L} 16$ binds directly to the cytoplasmic tail of $\mathrm{gD}$. If the cytoplasmic tail of $\mathrm{gD}$ is removed, $\mathrm{U}_{\mathrm{L}} 16$ is still packaged into virions [678]. This non-reciprocal interaction suggests that packaging of $\mathrm{U}_{\mathrm{L}} 16$ on 
capsids is independent of $\mathrm{gD}$, but recruitment of $\mathrm{gD}$ during cytoplasmic envelopment may require $\mathrm{U}_{\mathrm{L}} 16$.

\section{6. $U_{L} 53(g K)$ and $U_{L} 20$}

Glycoprotein $\mathrm{K}(\mathrm{gK})$ is a highly hydrophobic 338-amino-acid protein that is encoded by the $\mathrm{U}_{\mathrm{L}} 53$ gene [679]. gK is highly embedded on the membrane [680], which makes its study difficult. Its structure is composed of three or four transmembrane domains, as shown by tag insertions in multiple domains of gK [680].

Deletion of HSV-1 gK results in a small plaque phenotype, lower viral yields, and accumulation of non-enveloped virion particles in the perinuclear space [681,682]. These data suggest that $\mathrm{gK}$ has a role in the egress of virus from infected cells (Figure 3). Further work demonstrated that deletion of gK triggers a collapse of the Golgi to the ER, in a manner similar to brefeldin A [680]. Virion entrapment in this perinuclear space may occur due to this Golgi collapse, and it has been suggested that this collapse may be partially due to an antifusogenic role of gK during egress.

The antifusogenic role of gK has been studied extensively. Cell-to-cell transmission of HSV-1 occurs by either release of virions to the extracellular space or virus-induced cell-to-cell fusion. Certain spontaneous mutants of HSV-1 have been found to induce the formation of large multinucleated cells or syncytia. Such mutations have been identified in $\mathrm{gK}$ and in other viral glycoproteins, such as $\mathrm{gB}$, but those in $\mathrm{gK}$ are more frequently observed [683]. The syncytial gK mutants of HSV-1 have been used in multiple studies to investigate the function of gK during infection.

When $\mathrm{gK}$ is expressed outside the context of the infection, it localizes in the ER and the perinuclear space. However, infection of syncytial gK-transfected cells with a gK-null virus triggered expression of gK on the cell surface and cell fusion [680]. Wild-type gK can also inhibit fusion that is triggered by other HSV-1 glycoproteins outside the context of the infection. Co-transfection of the four viral glycoproteins $\mathrm{gD}, \mathrm{gB}, \mathrm{gH}$, and gL triggers cell-to-cell fusion and leads to syncytia. However, co-expression of wild-type gK with these glycoproteins reduces dramatically the formation of syncytia [684]. Therefore, it was suggested that gK is part of the mechanism through which HSV-1 regulates its own fusogenic activity. The anti-fusogenic activity of gK might prevent fusion of the viral envelope with the membrane of exocytic vesicles as the virus leaves the cell. In a similar manner, it could prevent the collapse of Golgi to the ER that was described above. BFA works by blocking vesicle and protein transport from the ER to the Golgi, and gB could affect Golgi integrity by blocking vesicles feeding into the Golgi from the ER [680].

The antifusogenic role of gK can also be seen during the absence of $\mathrm{U}_{\mathrm{L}} 20$ in $\mathrm{U}_{\mathrm{L}}$ 20-null virus-infected cells. This virus produced smaller plaques, and electron microscopy of infected cells showed accumulated capsids in the cytoplasm with few enveloped virions inside cytoplasmic vesicles [685]. A gK syncytial mutant in a $\mathrm{U}_{\mathrm{L}} 20$-null genetic background did not allow cell fusion as seen previously. Additionally, multiple virion capsids within a single envelope were seen in the cytoplasm, further supporting the anti-fusogenic role of $\mathrm{gK}$ during viral egress and also indicating an indirect role for $\mathrm{U}_{\mathrm{L}} 20$ in membrane fusion [685].

The $\mathrm{U}_{\mathrm{L}} 20$ gene encodes a 222-amino-acid non-glycosylated transmembrane protein that is conserved in all herpesviruses. It was thought that $U_{L} 20$ was essential for virus replication since deleting $U_{L} 20$ prevented replication. However, experiments utilizing $143 \mathrm{TK}^{-}$cell lines indicated cell type-dependent replication of $\mathrm{U}_{\mathrm{L}} 20$-null HSV-1 (F). Electron microscopy images of this virus in non-permissive Vero cells revealed a profound entrapment of viral particles in the perinuclear space. $U_{L} 20$ is required for intracellular transport and cell surface expression of $\mathrm{gK}$ in transient expression experiments, indicating a role in virus-specified glycoprotein trafficking. During infection, $\mathrm{U}_{\mathrm{L}} 20$ protein is required for $\mathrm{gK}$ transport to the surface, which is necessary for virus-induced cell fusion that is caused by syncytial mutations in either $\mathrm{gB}$ or $\mathrm{gK}$ (i.e., mutations in the $\mathrm{gB}$ and $\mathrm{gK}$ genes that allow for formation of syncytia) [685]. 
Additionally, gK is a virion component that is important for virus entry into cells [686]. The role of gK during entry might stem from its interaction with the viral glycoproteins that mediate virus entry. gK forms a functional protein complex with $\mathrm{U}_{\mathrm{L}} 20$, which is required for $\mathrm{gK}$ and $\mathrm{U}_{\mathrm{L}} 20$-associated functions in the life cycle of HSV-1 $[686,687]$. Coimmunoprecipitation experiments showed that $\mathrm{U}_{\mathrm{L}} 20$ forms a complex with $\mathrm{gB}$ and $\mathrm{gH}$ in infected cells but not with gD [688]. Additionally, gK has a functional amino-terminal domain [689] that can interact with the extracellular portions of $\mathrm{gB}$ and $\mathrm{gH}$ [688]. These results suggest that the $\mathrm{gK} / \mathrm{U}_{\mathrm{L}} 20$ complex may modulate the fusogenic properties of $\mathrm{gB}$ and $\mathrm{gH}$ via direct physical interactions. Treatment of virions with a protease that cleaves the gB-binding domain of gK results in reduced infectivity of the treated virions [690], which further supports the presence of $\mathrm{gK}$ on the surface of the virion and its role in mediating virus entry. Other data further support that $\mathrm{gK}$ is required for proper localization of $\mathrm{gD}$ and $\mathrm{gH} / \mathrm{gL}$ on HSV-1 assembly compartments [691]. Additionally, $\mathrm{gK}$ is required for $\mathrm{gB}$ binding to Akt during entry into neuroblastoma cells, release of calcium, and fusion of the viral envelope with host membranes. In the absence of the N-terminal functional domain of gK, entry into cells occurs through endocytosis [692]. Virus entry is therefore modulated by gK at multiple levels.

An interesting sequence of clinically relevant papers show the importance of $\mathrm{gK}$ in ocular infection with HSV-1 (Figure 4B). While deletion of the N-terminal domain of gK does not affect growth in Vero cells, it reduced cell-to-cell spread. Ocular infection of mice with a mutant HSV-1 that lacks the N-terminus of gK produced no significant ocular disease symptoms, versus infection with a wild-type strain. Additionally, the viral genome could not be amplified from ganglionic neurons that were infected with the mutant versus the wild-type HSV-1 [693]. Therefore, the N-terminus of gK is essential for neuroinvasiveness and herpes keratitis in the mouse ocular model. Work expanding on this paper showed that a virus lacking the $\mathrm{N}$-terminal domain of gK can attach to cell surfaces of Vero cells and ganglionic axons as efficiently as wild-type HSV-1; however, the mutant virus cannot enter into the cytoplasm of ganglionic neurons [692]. These data are in agreement with data showing decreased corneal scarring in ocularly infected mice with a gK mutant virus [694].

\section{7. $U_{L} 21$}

$\mathrm{U}_{\mathrm{L}} 21$ is an accessory gene that encodes a 535-aa protein of the tegument. It was reported by Baines et al. to be dispensable for viral replication in cell culture. $U_{L} 21$ promotes the growth of long cellular protrusions when over-expressed in non-neuronal cells and is associated with microtubules [695]. Additionally, $\mathrm{U}_{\mathrm{L}} 21$ forms a complex with $\mathrm{U}_{\mathrm{L}} 11, \mathrm{U}_{\mathrm{L}} 16, \mathrm{U}_{\mathrm{L}} 21$, and $\mathrm{gE}$ in transfected cells, and is necessary for the $\mathrm{U}_{\mathrm{L}} 11-\mathrm{U}_{\mathrm{L}} 16$ interaction [676].

$\mathrm{U}_{\mathrm{L}} 21$ is non-essential, but viral growth kinetics with a $\mathrm{U}_{\mathrm{L}} 21$-null virus showed that the overall viral yield is lower [696]. Most $\mathrm{U}_{\mathrm{L}} 21$-interacting proteins were found to be cytoskeletal proteins expressed in the central nervous system, such as the glial fibrillary acidic protein (GFAP). The distribution of GFAP is also altered in $\mathrm{U}_{\mathrm{L}}$ 21-null virus-infected glial cells, when compared to WT-virus-infected cells. These results suggest that $U_{L} 21$ is involved in capsid transport through interacting with cytoskeletal proteins. The altered distribution of GFAP has only been reported in glial cells, so it is not clear if $U_{L} 21$ can affect trafficking in neurons and no follow-up studies are available [696].

Infection with a $\mathrm{U}_{\mathrm{L}} 21$-null HSV-1 resulted in a delay in the onset of immediate early gene expression. Additionally, a reduced number of capsids were found in the cytoplasm after $\mathrm{U}_{\mathrm{L}}$ 21-null virus infection although DNA-containing capsids were formed in the nucleus [697]. These data suggest that $\mathrm{U}_{\mathrm{L}} 21$ has an early function that facilitates viral gene expression, as well as a late function that promotes the exit of capsids from the nucleus to the cytoplasm. The early function is supported by the crystal structure of the C-terminal domain of $\mathrm{U}_{\mathrm{L}} 21$ [698]. Based on these studies, it is shown that $\mathrm{U}_{\mathrm{L}} 21$ can bind E. coli RNA, which suggests a role for $\mathrm{U}_{\mathrm{L}} 21$ in transcription or translation, but further work is needed. Regarding the late function of $\mathrm{U}_{\mathrm{L}} 21$, multiple empty capsids have been observed in the 
cytoplasm of $U_{L}$ 21-null-infected cells [699]. Therefore, it was suggested that $U_{L} 21$ either retains capsids in the nucleus until they receive DNA, and disruption of $\mathrm{U}_{\mathrm{L}} 21$ allows empty capsids to be transported to the cytoplasm, or that $\mathrm{U}_{\mathrm{L}} 21$ protects DNA-filled capsids and its absence results in empty capsids in the cytoplasm [699].

\section{8. $U_{L} 24$}

$\mathrm{U}_{\mathrm{L}} 24$ is a late viral gene that is expressed as a predominantly nucleus-associated $30 \mathrm{kDa}$ protein [700] but localizes to the cytoplasm as well [701]. It is encoded by mRNAs with two different $5^{\prime}$ ends. The majority of $\mathrm{U}_{\mathrm{L}} 24$ is encoded by the mRNA that contains the first initiation codon of the ORF [702]. It is unclear why $\mathrm{U}_{\mathrm{L}} 24$ is transcribed from different sets composing six transcripts [702]. The third initiation codon in the $\mathrm{U}_{\mathrm{L}} 24$ ORF leads to the expression of a protein termed $\mathrm{U}_{\mathrm{L}} 24.5$ with a size of $18 \mathrm{kDa}$ [703]. A $\mathrm{U}_{\mathrm{L}} 24.5$-null HSV-1 exhibits viral growth similar to a WT virus but does not trigger dispersal of nucleolar proteins as WT [703].

A $U_{L}$ 24-null virus yields mildly lower titers in cells and slightly smaller plaque sizes. Corneal infection in mice with a $\mathrm{U}_{\mathrm{L}} 24$-null virus results in 1 log lower viral load versus $\mathrm{WT}$, but there is a 4 logs lower viral growth in the trigeminal ganglia. These data suggest that $\mathrm{U}_{\mathrm{L}} 24$ is important for the dissemination of HSV-1 from the cornea to the trigeminal ganglia in mice (Figure $4 \mathrm{~A}$ ) $[700,704]$. $\mathrm{U}_{\mathrm{L}} 24$ may be important for virulence in murine and guinea pig models of intravaginal infection with HSV-2; however, the similarities between HSV-1 and HSV-2 $\mathrm{U}_{\mathrm{L}} 24$ are difficult to assess [705].

$\mathrm{U}_{\mathrm{L}} 24$ is one of four genes that when mutated can confer a syncytial (syn) phenotype [701]. It is not known how mutations in the $\mathrm{U}_{\mathrm{L}} 24$ gene confer syncytia. Mechanistically, lack of $\mathrm{U}_{\mathrm{L}} 24$ during late infection results in mislocalization of $\mathrm{gB}$ and $\mathrm{gD}$ with respect to actin [701], which are proteins involved in fusion. $\mathrm{U}_{\mathrm{L}} 24$ mutations that confer syncytia may work through the effect of $U_{L} 24$ on the localization of these fusogenic viral glycoproteins.

$\mathrm{U}_{\mathrm{L}} 24$ was found through bioinformatics to contain PD-(D/E)XK endonuclease signature sequences [706]. These sequences are required for the dispersal of nucleolin that occurs during infection, since their deletion or mutagenesis prevents nucleolin dispersal that occurs normally during infection. This suggests that $U_{L} 24$ is involved in nucleolin dispersal through its endonuclease motif $[707,708]$. Mutating the endonuclease motif also causes one log lower viral growth in the eye and the trigeminal ganglia of an ocular mouse model, indicating that the effect of $\mathrm{U}_{\mathrm{L}} 24$ endonuclease function is involved in dissemination of HSV-1 from the eye to the ganglia in vivo [709]. Another nucleolar component that is dispersed due to the endonuclease function of $\mathrm{U}_{\mathrm{L}} 24$ is the B23 nucleolar protein [710], which is a multifunctional protein that participates in ribosome biogenesis, mRNA processing, chromatin remodeling, and maintains genome stability [711]. $\mathrm{U}_{\mathrm{L}} 24$ also mediates nuclear egress of HSV-1 nucleocapsids and this effect most likely occurs through the abovementioned effect of $\mathrm{U}_{\mathrm{L}} 24$ on dispersion of nucleolar proteins [712].

$\mathrm{U}_{\mathrm{L}} 24$ may also play a role in immune evasion, through a function unrelated to its endonuclease motif [713]. Exogenous $\mathrm{U}_{\mathrm{L}} 24$ can bind with the p65 and p50 components of NF- $\mathrm{kB}$ and prevent their translocation to the nucleus. Therefore, it impairs the production of IFN- $\beta$ and pro-inflammatory chemokines and may mediate immune evasion during HSV-1 infection [713].

\section{9. $U_{L} 31$ and $U_{L} 34$}

$\mathrm{pU}_{\mathrm{L}} 34$ is a type 2 integral membrane protein with a 247-aa nucleoplasmic domain that binds $\mathrm{pU}_{\mathrm{L}} 31$ and holds it in close approximation to the inner nuclear membrane (INM) $[714,715]$. $\mathrm{U}_{\mathrm{L}} 34$ is anchored to the INM by a C-terminal transmembrane helix, with several residues extending into the perinuclear space [714]. $\mathrm{U}_{\mathrm{L}} 34$ retention at the INM requires the presence of $U_{L} 31$ [716], and both $U_{L} 34$ and $U_{L} 31$ localization is dependent on their co-expression and interaction [562]. $\mathrm{U}_{\mathrm{L}} 31$ and $\mathrm{U}_{\mathrm{L}} 34$ form a complex, which has been 
termed the nuclear egress complex (NEC), and it is required for efficient exit of nascent HSV-1 capsids from the nucleus (Figure 3A).

Deletion of $\mathrm{U}_{\mathrm{L}} 31$ results in 3-4 logs lower viral yields compared to WT HSV-1, slightly decreased levels of total viral DNA, and a 3-5-fold reduction in the ratio of monomeric to concatemeric DNA, suggesting minor roles in both DNA replication and processing or packaging of viral DNA [717].

Deletion of $\mathrm{U}_{\mathrm{L}} 34$ results in 2-5 logs lower yields of HSV-1 in cell culture. While a $\mathrm{U}_{\mathrm{L}} 34$-null virus can assemble DNA-containing capsids, they accumulate in the nucleus and are unable to bud through the inner nuclear membrane [718].

The role of $\mathrm{U}_{\mathrm{L}} 31$ and $\mathrm{U}_{\mathrm{L}} 34$ initiates with the disruption of nuclear lamina during HSV1 replication. Formation of HSV-1 replication compartments (RCs) and annexation of space in the nucleus results in cellular chromatin marginalization and compression [719]. The phase of chromatin marginalization occurs during the initial phase of RC formation, and this does not require $U_{L} 31$ and $U_{L} 34$. However, later during infection, $R C$ s penetrate the host chromatin and the nuclear lamina, and reach a region of the nucleus close to the INM. In co-transfection experiments of $\mathrm{U}_{\mathrm{L}} 31$ and $\mathrm{U}_{\mathrm{L}} 34$, marginalization of host chromatin was not observed; however, during infection, both $\mathrm{U}_{\mathrm{L}} 31$ and $\mathrm{U}_{\mathrm{L}} 34$ are required for alteration of the distribution of lamina components [720], which suggests that other viral proteins cooperate with NEC for lamina disruption. Nonetheless, $\mathrm{U}_{\mathrm{L}} 34$ can interact directly with lamin $\mathrm{A} / \mathrm{C}$ in vitro [721]. This disruption of lamina is a regulated process during infection since the viral protein $U_{S} 3$ is involved $[557,721,722]$. The kinase activity of $U_{S} 3$ was not necessary for the redistribution and disruption of lamin A/C or lamin B [559], indicating that $U_{S} 3$ spatial interaction with NEC may be the regulatory mechanism. However, $U_{S} 3$ phosphorylates lamin A/C during HSV-1 infection [558].

The N-terminus of $\mathrm{pU}_{\mathrm{L}} 31$ also harbors multiple phosphorylation sites of the viral $\mathrm{U}_{\mathrm{S}} 3$ kinase. Preventing the phosphorylation of $\mathrm{pU}_{\mathrm{L}} 31$ mimics the growth defect of a $\mathrm{U}_{\mathrm{S}} 3$-null virus, with 1-2 logs lower viral yields. The importance of the $\mathrm{N}$-terminus and its phosphorylation is highlighted by the following observations: First, $\mathrm{pU}_{\mathrm{L}} 31$ that lacks the $\mathrm{N}$-terminus is retained in the cytoplasm if co-expressed with $\mathrm{U}_{\mathrm{L}} 34$, suggesting that they prematurely interact before they enter the nucleus. This is probably why $U_{L} 31$ and $\mathrm{U}_{\mathrm{L}} 34$ utilize different transport routes to the nucleus, averting their premature interaction. Second, the phosphorylation of the N-terminus of $\mathrm{pU}_{\mathrm{L}} 31$ is necessary for the proper localization of the $\mathrm{pU}_{\mathrm{L}} 31 / \mathrm{pU}_{\mathrm{L}} 34$ complex in the nuclear rim and the optimal egress of virions from the perinuclear space [560]. $\mathrm{U}_{\mathrm{L}} 31$ and $\mathrm{U}_{\mathrm{L}} 34$ distribution is even across the nuclear rim, but this requires $\mathrm{U}_{\mathrm{S}} 3$ expression [562]. In the absence of $\mathrm{U}_{\mathrm{S}} 3, \mathrm{U}_{\mathrm{L}} 31$ and $\mathrm{U}_{\mathrm{L}} 34$ localize in small punctate areas at the nuclear rim. This supports that $\mathrm{U}_{\mathrm{L}} 31$ and $\mathrm{U}_{\mathrm{L}} 34$ form a complex that accumulates at the nuclear membrane and plays an important role in HSV-1 nucleocapsid envelopment at the inner nuclear membrane. Absence of $U_{S} 3$ causes accumulation of capsids in nuclear membrane invaginations, delayed onset of virus production, and reduced virus titers [556]. $\mathrm{U}_{\mathrm{L}} 31$ and $\mathrm{U}_{\mathrm{L}} 34$ associate with perinuclear virions but not with extracellular virions, supporting the de-envelopment/re-envelopment model of viral egress [556].

NEC by itself is sufficient to drive the vesiculation of the nuclear envelope in transfected cells in the absence of any other viral proteins [723-725]. Using purified HSV-1 NEC components and synthetic liposomes, it was shown that NEC has an intrinsic ability to vesiculate membranes in vitro [726]. NEC formed a coat-like hexagonal lattice on the inner surface of the budded vesicles, which suggested that it vesiculated membranes without the help of other proteins by creating a hexagonal scaffold inside the bud [727]. Further structural characterization showed that HSV-1 $\mathrm{U}_{\mathrm{L}} 31$ and $\mathrm{U}_{\mathrm{L}} 34$ form the NEC heterodimer through extensive interactions that involve residues distributed throughout $U_{L} 31$ and $\mathrm{U}_{\mathrm{L}} 34$. The heterodimers are further organized to form oligomeric structures. Mutagenesis of their oligomeric interfaces reduced NEC-mediated budding in vitro, supporting that NEC oligomerization drives capsid budding during nuclear egress of herpesviruses [726]. 
However, other cellular and viral factors may still participate in NEC oligomerization and nuclear budding in cells, such as $\mathrm{U}_{\mathrm{S}} 3$ mentioned above.

Besides NEC oligomerization on the INM, a host factor mediating primary envelopment of HSV-1 is the endosomal sorting complex required for transport-III (ESCRT-III). ESCRT-III promotes primary envelopment by mediating scission during HSV-1 budding through the INM [728]. $\mathrm{U}_{\mathrm{L}} 34$ interacts with ALIX but not other ESCRT-III proteins, suggesting that ALIX acts as an adaptor for the recruitment of ESCRT-III proteins by $\mathrm{U}_{\mathrm{L}} 34$, resulting in scission of the budding vesicles formed by the NEC. The mechanism of fusion of the primary envelope with the outer nuclear membrane is still not clear.

$\mathrm{U}_{\mathrm{L}} 31$ also functions in another interesting manner in order to conserve viral resources. Three major types of HSV-1 capsids have been described, the empty capsids (A capsids), capsids that lack viral DNA (B capsids), and viral DNA-containing capsids (C capsids) [729]. Type C capsids are preferentially selected compared to A and B to undergo primary envelopment at the INM, and the mechanism of their selective involvement involves $U_{\mathrm{L}} 31$, $\mathrm{U}_{\mathrm{L}} 17$, and $\mathrm{U}_{\mathrm{L}} 25$. $\mathrm{U}_{\mathrm{L}} 17$ and $\mathrm{U}_{\mathrm{L}} 25$ interact and form a stable complex. The different types of capsids contain different copies of this complex. C capsids contain 75 copies, while B capsids contain 25 copies [730]. Because of its enrichment in C capsids, the $\mathrm{U}_{\mathrm{L}} 25 / \mathrm{U}_{\mathrm{L}} 17$ complex is termed C capsid-specific complex (CCSC). While it is possible that the CCSC binds more efficiently to $C$ capsids, an interaction has been identified between $U_{L} 31$ and CCSC in infected cells [731]. This supports a model of egress in which the CCSC is added to capsids after DNA is inserted and engages $\mathrm{U}_{\mathrm{L}} 31$ either in the nucleus, or within the NEC at the INM. The end result is an elegant way to conserve cellular resources by selecting only capsids that have the potential to produce infectious virions for primary envelopment [731].

\subsection{0. $U_{L} 35(V P 26)$}

The HSV-1 $\mathrm{U}_{\mathrm{L}} 35$ gene encodes for a 12-kDa capsid protein designated VP26, which is located on the outer surface of the viral capsid, on the tips of the hexons that constitute the capsid shell [732]. The HSV-1 capsid has an icosahedral structure, and the major capsid protein is VP5. VP5 forms both the pentons and the hexons of the capsid, which are composed of five or six VP5 monomers [733]. Pentons are located at the icosahedral vertices, while hexons form the faces and the edges of the capsid structure. VP26 is attached to VP5 molecules that make up the hexons. The C-terminus of VP26 interacts with the upper domain (UD) of VP5 [734], and their interaction has been well characterized [734,735]. VP5 and VP26 interaction is required for localization of VP26 to the sites of capsid assembly in the nucleus [736]. However, in vitro reconstitution of HSV-1 capsids showed that VP26 is not required for proper capsid assembly [732,737].

These data agree with reports that show that VP26 is non-essential for viral growth in vitro [738] but influences the production of infectious virus in vivo. In a mouse ocular infection, a $\mathrm{U}_{\mathrm{L}} 35$-null virus yields 2 -fold less virus in the eye but 30-100-fold less virus in the trigeminal ganglia (Figure 4A). VP26 does not seem to affect the transport of the virus from the eye to the ganglia but is important for the replication of the virus in the ganglia [738]. Similar results have shown in vitro that a $U_{L} 35$-null virus exhibits reduced viral yields when cultured in neuroblastoma cell lines. A potential reason is mislocalization of the main capsid protein VP5, which exhibits a punctate distribution during $\mathrm{U}_{\mathrm{L}}$ 35-null virus infection as opposed to a diffuse distribution during a WT infection [739].

Another way that VP26 can affect replication is by mediating incorporation of $\mathrm{U}_{\mathrm{L}} 25$ into nucleocapsids and by extension affecting DNA packaging. This is because $\mathrm{U}_{\mathrm{L}} 25$ is part of the 3-component viral terminase complex, which transports the HSV-1 genome into the viral capsid [740]. This is supported by yeast-two-hybrid data that show interaction of VP26 and $\mathrm{U}_{\mathrm{L}} 25$ [735].

VP26 may also affect capsid delivery to the nucleus following entry of the virus into the cells by interacting with the dynein light-chain subunits DYLNT1 and DYLNT3. Cytoplasmic dynein is a molecular motor that is associated with microtubules, and each dynein complex contains two copies of either DYLNT1 or DYLNT3. VP26, DYLNT1 and 
DYLNT3 colocalize with microtubules, and VP26 is required for migration of HSV-1 capsids towards the nucleus [741]. These data suggest that VP26 is important for the retrograde transport of HSV-1 capsids from the plasma membrane towards the nuclear membrane after viral entry into cells. Further work described the N-terminus of VP26 as a binding region for the dynein light-chain subunits DYNLT1 and DYNLT3 [742].

\subsection{1. $U_{L} 43$}

$\mathrm{U}_{\mathrm{L}} 43$ has a size of about $32 \mathrm{kDa}$ and based on its sequence, it may contain seven transmembrane domains composed almost entirely of alpha helixes [743]. $U_{L} 43$ is not present in mature extracellular virions [744]. Other functions remain unknown.

\subsection{2. $U_{L} 44(g C)$}

$\mathrm{gC}$ is a 511-aa type I integral membrane glycoprotein that mediates HSV-1 attachment to host cell surface glycosaminoglycans. Absence of $\mathrm{gC}$ results in reduced binding of virus to cells, although the virus that binds can enter cells and initiate infection [745]. gC can bind to both heparin and heparan sulfate. The binding that occurs in the absence of $\mathrm{gC}$ is dependent on cell surface heparan sulfate $[745,746]$. It was shown that two areas of gC participate in heparan sulfate binding (R143, R145, R147, T150, G247). Synthetic peptides that corresponded to these two areas prevented virus binding and entry in cells, and they also agglutinated red blood cells [747]. These data suggest that these $\mathrm{gC}$ areas mediate the binding of virus on cell surface heparan sulfate.

Additionally, gC can regulate cell entry and infection by a low-pH pathway [748]. The presence of $\mathrm{gC}$ confers a higher $\mathrm{pH}$ threshold for acid-induced changes in $\mathrm{gB}$, affecting fusion. Using a $\mathrm{gC}$-null virus, it was found that there was a delay in entry relative to WT HSV-1 [745]. A research group tested infection with HSV-1 and gC-null HSV-1 on different cell lines. They observed that a gC-null virus displayed different infectivity, depending on whether these cell lines support low-pH or pH-neutral entry. Treatment with ammonium chloride did not affect gC-null HSV-1 entry into cells that support the $\mathrm{pH}$ neutral pathway, suggesting that $\mathrm{gC}$ is dispensable for that pathway. When they assessed for infectious virus after infection in a low-pH environment, gC-null HSV-1 was lagging in intracellular transport or release from intracellular vesicles formed after endocytic entry. After treating HSV-1 $\triangle \mathrm{gC}$ versus WT HSV-1 virions with different $\mathrm{pH}$ solutions, it was shown that the presence of $\mathrm{gC}$ increases the $\mathrm{pH}$ at which fusogenic conformational change of $\mathrm{gB}$ occurs [748].

\subsection{3. $U_{L} 45$}

$\mathrm{U}_{\mathrm{L}} 45$ is a late gene [749] that encodes an $18-\mathrm{kDa}$ protein that is present in virions and is enriched in the envelope-tegument interface, thus associated with the viral envelope [749]. While it is non-essential for viral growth in vitro [750], it is required for efficient growth in the central nervous system (CNS) of mice when inoculating with a low viral dose [751].

$\mathrm{U}_{\mathrm{L}} 45$ is required for syncytia formation during infection with a gB mutant HSV-1 that causes syncytia (gBsyn) [752]. A UL45 C-terminus truncation prevents the formation of syncytia with gBsyn [753], suggesting that $\mathrm{U}_{\mathrm{L}} 45$ affects entry that requires $\mathrm{gB}$ function. However, $\mathrm{U}_{\mathrm{L}} 45$ plays a dispensable role in virus entry to cells either through $\mathrm{pH}$-dependent endocytosis or $\mathrm{pH}$-independent mechanisms [754].

\subsection{4. $U_{L} 55$ and $U_{L} 56$}

The $\mathrm{U}_{\mathrm{L}} 56$ gene product is a C-terminal-anchored type II membrane protein conserved among HSV-1, HSV-2, and herpes B virus. Even though $\mathrm{U}_{\mathrm{L}} 56$ is dispensable for viral growth in cultured cells, it plays an important role in the pathogenicity of HSV-1.

HSV-1 mutants lacking $U_{\mathrm{L}} 56$ are substantially less pathogenic in mice but have similar growth in cell culture [755]. The role of $\mathrm{U}_{\mathrm{L}} 56$ in the severity of HSV-1 ocular disease can be seen in work based on a quantitative trait locus (QTL)-based assay, which involved infecting mice with 40 recombinant strains derived from mice infected simultaneously 
with two avirulent strains. Phenotypically meaningful variations could be seen in multiple genes, including $\mathrm{U}_{\mathrm{L}} 56$, whose features were associated with an increase in ocular virulence in mice [756]. However, $\mathrm{U}_{\mathrm{L}} 55$ and $\mathrm{U}_{\mathrm{L}} 56$ do not appear to have a role in the latent stage of the virus since mice that were infected with HSV-1 lacking $\mathrm{U}_{\mathrm{L}} 55$ and $\mathrm{U}_{\mathrm{L}} 56$ could still develop a latent infection [757]. Furthermore, HSV-1 mutants lacking the entire $\mathrm{U}_{\mathrm{L}} 56$ gene have been found in human samples [758] and are considered to be less pathogenic or to lack neurovirulence $[755,758]$. Therefore, $\mathrm{U}_{\mathrm{L}} 56$ is non-essential in cell culture, and is not implicated in latency but is important for pathogenicity in vivo.

$\mathrm{U}_{\mathrm{L}} 56$ contains a hydrophobic domain in its carboxyl-terminal tail (aa 217-234) which is embedded in the membrane, and its deletion results in reduced pathogenicity as it generates an avirulent HSV-1 strain [759]. Characterization of $U_{L} 56$ through immunofluorescence studies showed that $\mathrm{U}_{\mathrm{L}} 56$ localized to the Golgi and cytoplasmic vesicles in $\mathrm{U}_{\mathrm{L}} 56$-transfected or HSV-2-infected cells. The C-terminal domain is important for association with cytoplasmic membranes and the $\mathrm{N}$-terminal is important for its translocation to the Golgi and the cytoplasmic vesicles. Protease digestion assays combined with fractionation through sucrose gradients suggested that $\mathrm{U}_{\mathrm{L}} 56$ is a type II membrane protein associated with lipid rafts. These data suggest that $\mathrm{U}_{\mathrm{L}} 56$ may be involved in vesicular trafficking in HSV-2-infected cells. Expanding on that work, an interaction between $\mathrm{U}_{\mathrm{L}} 11$ and $\mathrm{U}_{\mathrm{L}} 56$ was identified [760], suggesting a complex that may be involved in the cytoplasmic envelopment of HSV.

Ushijima et al. in a series of papers investigated the role of $U_{L} 56$ during HSV-2 infection. They first demonstrated that $\mathrm{U}_{\mathrm{L}} 56$ interacts through its PY motifs with Nedd4, an E3 ubiquitin ligase. $\mathrm{U}_{\mathrm{L}} 56$ triggered increased NEDD4 ubiquitination and its subsequent degradation during infection [761]. Additionally, they investigated potential co-localization of $\mathrm{U}_{\mathrm{L}} 56$ with Nedd4 and they found that $\mathrm{U}_{\mathrm{L}} 56$ localizes to the TGN and early endosomes but not with $\mathrm{CD} 63$ in late endosomes. Co-localization of $\mathrm{U}_{\mathrm{L}} 56$ with Nedd4 was observed at the TGN, but a lack of co-localization with CD63 suggested that HSV-2 is not using MVBs for cytoplasmic envelopment. Nonetheless, deletion of $\mathrm{U}_{\mathrm{L}} 56$ restricted infectious HSV-2 release. Therefore, Ushijima suggested that $\mathrm{U}_{\mathrm{L}} 56$ functions in coordination with other host or viral factors in trafficking and membrane sorting. Nedd4 may be among these factors, and this function of $\mathrm{U}_{\mathrm{L}} 56$ may be redundant with other viral proteins that have sorting functions and may also depend on the cell type [762].

Ushijima et al. in 2010 showed how $\mathrm{U}_{\mathrm{L}} 56$ interacts with Itch, which is another Nedd4family ligase, triggering its degradation through lysosomes. Interestingly, HSV-1 does not degrade Nedd4, but it does degrade Itch [763].

\subsection{5. $U_{S} 2$}

The $\mathrm{U}_{\mathrm{S}} 2$ gene of HSV is predicted to encode a 291 -aa protein of $33 \mathrm{kDa}$. It is predicted to have a hydrophobic N-terminus [764]; it is non-essential in cell culture and not involved in the pathogenesis in the CNS of mice [765]. HSV-1 Us2 is not associated with any specific phenotype [766]; however, most research so far has been done on HSV-2 Us2. Initially, $\mathrm{U}_{\mathrm{S}} 2$ was observed as discrete granules late during infection within and at the periphery of the nucleus [767]. However, further analysis of $U_{S} 2$ by immunofluorescence microscopy of infected Vero and A431 cells detected a filamentous-like cytoplasmic pattern [768]. Additional data suggested interaction of $\mathrm{U}_{\mathrm{S}} 2$ with cytokeratin 18 through yeast-two-hybrid assays, and confirmed the interaction by co-immunoprecipitation, which seems to involve the N-terminus of $U_{S} 2$ [768]. Other HSV gene products can also interact with proteins of the cytoskeleton [769-771]. $\mathrm{U}_{\mathrm{S}} 2$ and cytokeratin 18 interaction suggests participation of $\mathrm{U}_{\mathrm{S}} 2$ in trafficking during infection, but more work is needed to characterize this function.

It was later shown that HSV-2 $\mathrm{U}_{\mathrm{S}} 2$ is a membrane-associated ubiquitin-interacting protein [772]. HSV-2 U 2 lacks specific membrane sorting signals, and can be found at the plasma membrane, in cytoplasmic vesicles, and diffusely throughout the cytoplasm. Through a discontinuous gradient, $\mathrm{U}_{\mathrm{S}} 2$ can be detected in detergent-resistant membranes, and cofractionates with caveolin-1 and ganglioside GM1. Treatment of infected cells with 
BFA (brefeldin A) did not affect the localization of $U_{S} 2$, suggesting that it is not part of the ER-Golgi secretory pathway [772]. Co-localization experiments showed that $\mathrm{U}_{\mathrm{S}} 2$ localizes predominantly to recycling endosomes and the plasma membrane, but the resistance to BFA treatment suggests that this localization of $U_{S} 2$ is regulated post-translationally. Through mass spectrometry, most $\mathrm{U}_{\mathrm{S}} 2$-interacting proteins were shown to be ubiquitinated. $\mathrm{U}_{\mathrm{S}} 2$ could be pulled down by mono-ubiquitin conjugated agarose but not by protein $\mathrm{G}$ agarose, suggesting that $\mathrm{U}_{\mathrm{S}} 2$ interacts specifically with ubiquitin and not with ubiquitin-conjugated proteins [772].

Lu et al. in 2017 showed that HSV-2 $U_{S} 2$ could activate NF-kB signaling. Deficiencies in $\mathrm{U}_{\mathrm{S}} 2$ decreased HSV-2 WT-mediated NF- $\mathrm{KB}$ activation and cytokine and chemokine production, while overexpression of $U_{S} 2$ produced the opposite effects. Co-immunoprecipitations suggested that $\mathrm{U}_{\mathrm{S}} 2$ interacts with TGF- $\beta$-activated kinase 1 (TAK1). $\mathrm{U}_{\mathrm{S}} 2$ induced the phosphorylation of TAK1, resulting in the activation of TAK1-mediated downstream signaling. This role of $\mathrm{U}_{\mathrm{S}} 2$ in NF- $\mathrm{kB}$ activation was confirmed in mice. Interestingly, HSV-1 $\mathrm{U}_{\mathrm{S}} 2$ did not activate NF-kB like HSV-2 US2 [773].

\subsection{6. $U_{S} 4(g G)$}

Glycoprotein $\mathrm{G}(\mathrm{gG})$ is one of the least well-characterized glycoproteins of HSV-1. Infections with gG-null HSV-1 exhibit similar growth to WT virus and little attenuation in vivo [774]. When focusing on polarized epithelial cells though, $\mathrm{gG}$ seems to be required for infection through the apical surface. However, a gG-null virus can still infect these cells through the basal membranes and replicate normally. In vivo infection of apical surfaces of mouse corneas with a gG-null HSV-1 results in delayed scarification, but once scarification occurs, a gG-null virus has yields similar to wild-type virus [775].

An interesting observation is that inoculation of mice with a baculovirus recombinant vector carrying the gG ORF results in partial protection from lethal challenge with intraperitoneally injected HSV-1 [776,777]. This protection was not observed when using vaccinia virus as a vector [778]. This might be a result of higher expression of $\mathrm{gG}$ in the baculovirus system, or more intriguingly, $\mathrm{gG}$ in insect cells of the baculovirus system may be glycosylated in a different pattern that increases gG immunogenicity. However, this protection does not apply to corneal infection with HSV-1 after vaccination with the same gG-containing baculovirus vector [779].

The most interesting role of gG regards its interplay with chemokines. Chemokines are chemotactic cytokines that coordinate the recruitment of immune cells to infection sites, thus are important for the outcome of a viral infection [780]. Mice that are depleted of chemokine ligands or receptors are highly susceptible to genital herpes infection and neuroinvasion of the CNS due to defective leukocyte mobilization to the infected mucosa [781].

HSV-1 gG localizes on the plasma membrane [782], and it can bind chemokines with high affinity $[783,784]$. Binding of HSV-1 gG to chemokines while being on the plasma membrane of infected cells occurs through the glycosaminoglycan (GAG)-binding domain of the chemokine, which is required for binding to gG. Interestingly, binding of gG to chemokines does not inhibit chemokine function, rather increases it both in vitro and in vivo. Experiments show that higher migration of leukocytes occurs in the presence of gG on HSV-1-infected cells, due to increased chemokine binding to its specific receptor and downstream MAPK signaling mediated by the surface gG. It is possible that gG acts as a GAG and mediates a local increase of chemokine concentration in parts of the membrane. This will increase chemokine signaling, which will be beneficial for the virus in a number of ways [783]. First, it is possible that chemokine deregulation due to binding to gG promotes viral dissemination through MAPK signaling and NF- $\mathrm{kB}$ activation, which enhances viral replication [785]. An alternative hypothesis is that the increased infiltration of leukocytes to sites of infection increases the number of available cells that can then be infected by HSV-1, thus helping the virus spread in vivo [783]. The increased leukocyte migration hypothesis may still occur even through chemokines binding to HSV-1 particles, since gG is also present on the viral envelope [782,786]. 


\subsection{7. $U_{S} 5(g J)$}

$\mathrm{U}_{\mathrm{S}} 5$ is a late gene that encodes the glycoprotein J (gJ) of HSV-1. gJ localizes to multiple membrane compartments and has been little characterized [787]. $\mathrm{gJ}$ is another glycoprotein that mediates protection from CTL killing of infected cells. CTLs kill targets in part by inducing apoptosis either though activation of the Fas pathway and downstream activation of caspases or by releasing lytic granules that contain granzyme B, which can induce apoptosis by cleaving caspases in target cells. gJ can inhibit both pathways; however, other viral genes can compensate for deletion of gJ [788]. $\mathrm{gJ}$ can also bind the $\mathrm{F}_{0} \mathrm{~F}_{1}$ ATPase synthase in the mitochondrial membrane that is required for induction of ROS, which suggests that $\mathrm{gJ}$ may inhibit $\mathrm{F}_{0} \mathrm{~F}_{1}$ ATPase function [788].

\subsection{8. $g E / g I\left(U_{S} 8 / U_{S} 7\right), U_{S} 9$}

Glycoprotein $\mathrm{E}$ ( $\mathrm{gE}$ ) was first described as a receptor for the Fc portion of immunoglobulin $\mathrm{G}$ and for its role in virus spread from cell to cell [789]. Glycoprotein I (gI) is the other polypeptide of the gE/gI complex [789], and like gE, it contains a 400-aa extracellular (ET) domain and a 100-aa cytoplasmic (CT) domain. Since the majority of $\mathrm{gE}$ is bound to gI, these proteins are often studied in tandem.

gI can increase the affinity of gE to IgG. gE and gI mutants exhibit a small plaque phenotype in vitro compared to WT HSV-1 [774]. The number of plaques detected is not affected, suggesting that $\mathrm{gE}$ and gI regulate cell-to-cell spread in vitro [790]. Expression of $\mathrm{gE} / \mathrm{gI}$ in human epithelial cells resulted in the localization of $\mathrm{gE} / \mathrm{gI}$ at lateral surfaces of cells and colocalization with the adherens junction marker $\beta$-catenin. At subconfluent monolayers during infection, $\mathrm{gE} / \mathrm{gI}$ localize at the parts of the plasma membrane that are in contact with another cell [791]. Therefore gE/gI seems to mediate cell-to-cell spread of HSV-1 across cell junctions by interacting with cell junction components (Figure 3D).

Normally, HSV-1 particles are sorted to cell junctions, whereas few virions reach the apical surfaces of polarized epithelial cells [792]. Deleting gE results in HSV-1 virions that cannot translocate to cell junctions and they leave the cell through the apical surface [792]. Work that has characterized a panel of gE mutant viruses with small insertions in the ET domain underlined the importance of this domain of $\mathrm{gE}$ for cell-to-cell spread. Several of these gE ET mutant proteins were able to complex with gI and be incorporated into virions, but the formed virions behaved similarly to gE-null mutants. This suggests that $\mathrm{gE} / \mathrm{gI}$ promotes HSV-1 cell-to-cell spread by binding either extracellular ligands through the $\mathrm{gE}$ ET or components of cell junctions (Figure 3D).

Antibodies specific for HSV-1 antigens can simultaneously bind at the surface of infected cells to gE/gI via their Fc region and to a cell surface HSV-1 antigen by their antigen-binding fragments (Fabs) [793-795]. This process is known as antibody bipolar bridging (ABB), and may be a strategy to prevent the host from utilizing anti-HSV-1 antibody responses.

HSV-1 gE mutants show decreased neurovirulence [796], and the Fc binding portion of gE was shown to be important in vivo [797]. Introducing insertions in HSV-1 either inside or outside the Fc binding portion of $\mathrm{gE}$ resulted in lower yields only for the Fc domain mutant, when testing the two mutants in mice. Additionally, the Fc-binding gE mutant virus was impaired in its ability to reach the ganglia (Figure 4A) [797]. HSV-1 gE/gI-null mutants also show significantly reduced spread in the corneal epithelium of infected mice, due to the reduced ability of these mutants to undergo anterograde transport from sensory ganglia back to the cornea [798].

A major factor for the decreased neurovirulence and cornea infection of $\mathrm{gE}$ mutants is the less efficient anterograde transport (Figure 4A). Anterograde transport in infected neurons (e.g., after reactivation of virus from latency) involves the transport of viral particles from the neuronal-cell body along axons to axonal termini, and transfer across junctions formed between neurons and epithelial cells. gE and gI mutants displayed markedly reduced anterograde spread between neurons within the retina and from the retina to retinorecipient regions of the brain [799]. HSV-1 $\mathrm{gE} / \mathrm{gI}$ and $\mathrm{U}_{\mathrm{S}} 9$ possess overlapping or 
additive effects in anterograde axonal transport. Mutants lacking gE, gI, or $\mathrm{U}_{\mathrm{S}} 9$ displayed significantly reduced transport of capsids and glycoproteins towards axonal termini, while concomitant deletion of $\mathrm{gE}$ and $\mathrm{U}_{\mathrm{S}} 9$ produced nearly zero levels of capsids and glycoproteins even in proximal axons $[800,801]$. Investigation of HSV-1 infection using a mouse retina model concluded that HSV-1 $\mathrm{U}_{\mathrm{S}} 9$ is required for the transport of capsids, but not viral glycoproteins, from the retina into the optic nerve (Figure 4D) [802]. US9 is a viral tegument protein [803] that has been found associated with the ER and the Golgi [802], and with unenveloped capsids. It is tail anchored, has no ectodomain, and contains a cytoplasmic domain with TGN localization signals, which are important for its function $[804,805]$.

The fact that $\mathrm{gE}$ and $\mathrm{U}_{\mathrm{S}} 9 \mathrm{HSV}-1$ mutant viruses accumulate in the cytoplasm and do not enter the axons suggests either a trafficking issue or defective virion envelopment. Further characterization of the assembly of those mutants showed accumulation of unenveloped capsids in the cytoplasm of the infected cells and few enveloped virions. Both cannot enter axons in neuronal cells. Additionally, most capsids produced from gE and Us9 mutant viruses remained adhered to, or nested up against, membranes in the cytoplasm [806]. Considering that $\mathrm{gE} / \mathrm{gI}$ and $\mathrm{U}_{\mathrm{S}} 9$ accumulate in the TGN (a site of virus assembly), and that the $\mathrm{gE} / \mathrm{gI}$ complex sorts virus particles to epithelial cell junctions [792], the loss of $\mathrm{gE} / \mathrm{gI}$ and $\mathrm{U}_{\mathrm{S}} 9$ might lead to misrouting of HSV-1 capsids and virions so that they do not enter axons. Alternatively, the defective cytoplasmic envelopment might also inhibit anterograde transport. These defects may depend both on $\mathrm{gE}$ and $\mathrm{U}_{\mathrm{S}} 9$ since $\mathrm{U}_{\mathrm{S}} 9$ has been shown to colocalize with capsids and not glycoproteins, whereas $\mathrm{gE} / \mathrm{gI}$ colocalized with glycoproteins and not capsids [800].

It was also reported that gI can induce the formation of rod-shaped structures [807]. About $40 \%$ of gI-transfected cells expressed rod-shaped structures in the cytoplasm, besides the typical gI localization patterns (nuclear rim and cytoplasmic speckles, and junctions). The rods themselves vary in width and length. By doing immunofluorescence analysis using antibodies against different domains of gI, it was shown that the aa 110-202 of $\mathrm{gI}$ in the rod-shaped structures are not exposed. Since gE interacts with the aa 128-145 of gI, it will not interact with the gI of the rod-shaped structures, suggesting that $\mathrm{gE}$ is important for the proper function of gI. Two proline residues have been implicated in the induction of the gI rods, but they are not required for viral replication. However, these residues are important for mediating syncytia formation during infection with a $\mathrm{U}_{\mathrm{L}} 24$ mutant virus [807]. Thus, the coordination between gI and $\mathrm{U}_{\mathrm{L}} 24$ may be important for viral cell-to-cell spread and pathogenesis.

\subsection{9. $U_{S} 8.5$}

The $U_{S} 8.5$ gene overlaps with parts of the $U_{S} 8$ and the $U_{S} 9$ gene [808]. Its transcription is initiated within the coding sequence of $U_{S} 8$ and it is transcribed earlier than $U_{S} 8$, while the $U_{S} 8.5$ transcript is co-terminal with the transcripts of $U_{S} 8$ and $U_{S} 9$ [809]. The $U_{S} 8.5$ protein localizes in the nucleoli, but its function remains unknown.

\subsection{0. $U_{S} 10$}

The $U_{S} 10$ gene encodes a polypeptide of 313 aa [764] that was identified as a capsid/ tegument-associated protein localizing to nuclei as foci late during infection [810]. Deletion of $U_{S} 9,10,11$, or 12 has no effect on the neurovirulence and latency/reactivation potential of HSV-1, but it affects its neuroinvasiveness from peripheral sites to the CNS [810]. Fractionation studies show $\mathrm{U}_{\mathrm{S}} 10$ tightly associating with the nuclear matrix. Analysis of isolated intracellular capsids showed that both phosphorylated and unphosphorylated forms of $U_{S} 10$ were associated with the capsid/tegument. The nature of this association remains to be determined.

\subsection{1. $U_{S} 11$}

$\mathrm{U}_{\mathrm{S}} 11$ was identified as a late gene since it was shown that its expression required DNA replication [811]. Early work described the RNA-binding activity of $U_{S} 11$ to an in vitro RNA 
transcript of HSV-1 [812], which was later shown to be the $\mathrm{U}_{\mathrm{L}} 34$ transcript. $\mathrm{U}_{\mathrm{S}} 11$ binding to $\mathrm{U}_{\mathrm{L}} 34 \mathrm{mRNA}$ prevented its accumulation [813]. Expression of $\mathrm{U}_{\mathrm{S}} 11$ in baby hamster kidney cells prevented HSV-1 infection through a gD-mediated step, but this effect was not clarified [814]. Simonin et al. in 1995 [815] showed $U_{S} 11$ is phosphorylated independently of viral genome expression, by host kinases. The first work that connected $U_{S} 11$ and PKR showed that $\mathrm{U}_{\mathrm{S}} 11$ can bind protein kinase $\mathrm{R}$ in vitro and preclude the phosphorylation of eIF-2 $\alpha$ [319]. Work by the same group showed that $U_{S} 11$ and PKR interact in the context of viral infection and this interaction is RNA dependent (Figure 2D) [816].

Us11 is an abundant tegument protein of the virus that is released in the cells during virus entry. It can later be found in ribonucleoprotein fibrils, clusters of interchromatin granules, and in nucleoli [817]. Importantly, $\mathrm{U}_{\mathrm{S}} 11$ distribution in the nucleus follows that of nucleolin. Additionally, nuclear egress of HSV-1 capsids requires nucleolin, and $\mathrm{U}_{\mathrm{S}} 11$ and nucleolin associate during infection. The polyproline type II helix-containing domain of $\mathrm{U}_{\mathrm{S}} 11$ is required for this interaction, and this domain is also responsible for $\mathrm{U}_{\mathrm{S}} 11$ nucleolar accumulation. Nucleolin is involved in nucleocytoplasmic shuttling and $U_{S} 11$ accumulates in the nucleolus in its absence [817]. These data suggest that nucleolin could regulate the nucleocytoplasmic shuttling of $\mathrm{U}_{\mathrm{S}} 11$ during infection.

Interesting work showed that $\mathrm{U}_{\mathrm{S}} 11$ protein can bind HTLV-1 and HIV-1 responsive elements and can transactivate envelope retroviral glycoprotein expression by binding to the Rex-responsive element (RexRE), which is located in the $3^{\prime}$ untranslated region (UTR) of the HTLV-1 env mRNA [818]. Such data raise the possibility of in vivo interactions between herpes virus and human retroviruses, possibly affecting the expression of each virus at the post-transcriptional level. Further work showed how $\mathrm{U}_{\mathrm{S}} 11$ binds to HSV-1 mRNAs, such as the HSV-1 $\mathrm{U}_{\mathrm{L}} 34 \mathrm{mRNA}$ (its natural target), which results in its accumulation and might affect its trafficking. Two different $\mathrm{U}_{\mathrm{S}} 11$ domains were described, a C-terminal RNA-binding domain and an N-terminal effector domain, the deletion of which created a trans-dominant negative mutant [819].

More work on the binding partners of $\mathrm{U}_{\mathrm{S}} 11$ identified binding between the RNAbinding domain of $U_{S} 11$ and a 600-bp RNA sequence that is present in the co-terminal HSV-1 mRNAs $\mathrm{U}_{\mathrm{L}} 12, \mathrm{U}_{\mathrm{L}} 13$, and $\mathrm{U}_{\mathrm{L}} 14$ [600]. $\mathrm{U}_{\mathrm{S}} 11$ downregulates expression of the $\mathrm{U}_{\mathrm{L}} 13$ protein kinase at early times during infection [600]. $\mathrm{U}_{\mathrm{L}} 13$ is expressed with late gene kinetics but is also a component of the tegument. In the absence of $U_{L} 13$, there is a decrease in the accumulation of a subset of late mRNAs. $U_{S} 11$ might downregulate $U_{L} 13$ during early infection to stall the accumulation of the late mRNAs. These data further support the post-transcriptional control that $\mathrm{U}_{\mathrm{S}} 11$ applies to viral transcripts.

The subject of multiple studies has been the interaction of $U_{S} 11$ with PKR. The PKR kinase is an RNA sensor that upon sensing viral RNAs, phosphorylates the alpha subunit of the translation initiation factor eukaryotic initiation factor 2 (eIF2 $\alpha$ ) and thereby inhibits protein synthesis. The viral proteins $\gamma_{1} 34.5$ and $U_{S} 11$ prevent the accumulation of phosphorylated eIF $2 \alpha$ and consequently the translational shutoff. Particularly, the $\gamma_{1} 34.5$ protein directs protein phosphatase $1 \alpha$ to dephosphorylate eIF $2 \alpha$, reversing the effects of PKR activation (Figure 3D). Us11 when expressed under an immediate early promoter can rescue the growth of a $\gamma_{1} 34.5$-null virus. This requires a 68 -amino-acid fragment of $\mathrm{U}_{\mathrm{S}} 11$, which contains the RNA binding domain and was found to be sufficient for preventing PKR activation, thereby allowing protein synthesis and rescuing the growth of $\gamma_{1} 34.5$ mutant viruses [820]. This 68-aa domain of $U_{S} 11$ can also inhibit activation of PKR in a cell-free system, supporting the RNA binding function that has been ascribed to $\mathrm{U}_{\mathrm{S}} 11$. Through its interaction with PKR, $\mathrm{U}_{\mathrm{S}} 11$ can also inhibit autophagy activation by dsRNAs in a Beclin-1-independent manner [821].

The protein activator of PKR (PACT) is another restriction factor for HSV-1 as HSV1-induced interferon production in murine cells was inhibited in the absence of PACT. Binding of PACT to PKR is a dsRNA-independent mechanism of activation of PKR. PACTmediated PKR activation occurs only under cellular stress, such as withdrawal of growth factors or treatment with a low dose of actinomycin D [822]. US11 can prevent PACT- 
mediated PKR activation by binding to the dimerization domain (DD) of PKR. This allows binding of PACT to PKR but prevents the conformational change of PKR that is normally induced by PACT and activates PKR [823]. $\mathrm{U}_{\mathrm{S}} 11$ was found to bind both to PKR and to PACT, but only its binding to PKR was essential for preventing PACT from activating PKR, although binding of PACT to PKR was not prevented [824]. Further work on the binding of $U_{S} 11$ to PACT suggested that it might also prevent PACT-mediated activation of RIG-I (Figure 2D) [824].

$\mathrm{U}_{\mathrm{S}} 11$ also has a proviral role through inhibiting the synthesis of $2^{\prime}-5^{\prime}$ oligoadenylate by the oligoadenylate synthase (2'-5'-OAS) (Figure 2D). 2'-5'-OAS is another dsRNA sensor that acts to block protein synthesis and decreases RNA stability in virus-infected cells. Following RNA binding, 2'-5'-OAS can synthesize 2' -5 ' oligoadenylates (OA) from ATP that activate RNase L. Subsequently, RNase L cleaves mRNAs and rRNAs, inhibiting virus infection. HSV-1 can inhibit OA synthesis in IFN-stimulated primary human cells through the action of $U_{S} 11$. This inhibition requires the RNA-binding motif of $U_{S} 11$, which suggests that the mechanism involves partitioning of RNAs during infection [825].

$\mathrm{U}_{\mathrm{S}} 11$ can also counteract type I IFN activation by binding to Hsp90 and preventing TBK1 binding (Figure 2B). Consequently, downstream IRF3 phosphorylation and IFN induction are inhibited [826]. Further work from the same research group showed that $U_{S} 11$ also binds to the tripartite motif protein 23 (TRIM23), which is a key regulator of autophagymediated antiviral defense mediated by TBK1. The formation of autophagosomes mediated by TRIM 23 or TBK1 is reduced by $\mathrm{U}_{\mathrm{S}} 11$ in infected cells, through the exclusion of TBK1 from the TRIM23 complex in infected cells, in mouse embryonic fibroblasts (Figure 2C) [826]. It should be noted, however, that the exclusion of TBK1 might depend on cell type, especially since TBK1 is implicated in human fibroblasts in the modulation of autophagy [827].

$\mathrm{U}_{\mathrm{S}} 11$ is also involved in anterograde transport of HSV-1 in dorsal root ganglia (DRG). Kinesin is a microtubule-dependent molecular motor in cells that is used for transport of unenveloped HSV-1 nucleocapsids. US11 was identified as a kinesin-binding protein, and an interaction between $\mathrm{U}_{\mathrm{S}} 11$ and the heavy chain of kinesin (uKHC) was described [828]. The 20 to 24 RXP repeats in the carboxy half of $U_{S} 11$ that bind RNA are also where uKHC can bind. This polyproline domain may acquire a type II helix conformation with arginine residues on one side that interact with the negatively charged RNA. The other side contains hydrophobic, uncharged, or acidic chains that may provide specificity to the RNA binding and contain the $\mathrm{uKHC}$ binding site [829]. Another $\mathrm{U}_{\mathrm{S}} 11$ binding protein is the cellular PAT1 polypeptide, which binds microtubules, is involved in the intracellular trafficking of amyloid precursor protein (APP), and contains a region homologous to kinesin light chain (KLC). The $\mathrm{U}_{\mathrm{S}} 11-\mathrm{PAT} 1$ interaction also requires the carboxy-terminal RNA-binding domain of $U_{S} 11$ [830]. This association of $U_{S} 11$ with another molecular motor-associated protein further suggests a role for $\mathrm{U}_{\mathrm{S}} 11$ in trafficking of unenveloped capsids.

The effect of $U_{S} 11$ on neurovirulence has been further investigated in vitro and in vivo [831]. Intracranial infection of mice with a $U_{S} 11$-null virus is pathologically like a wild-type infection. In contrast, corneal infection with a $U_{S} 11$-null virus requires a longer time for onset of morbidity, indicating a role for $U_{S} 11$ in neuroinvasion. Replication in trigeminal ganglia and periocular tissue was mediated by $\mathrm{U}_{\mathrm{S}} 11$. However, $\mathrm{U}_{\mathrm{S}} 11$ deletion does not affect latency and the frequency of reactivation from trigeminal ganglia, even though the $U_{S} 11$-null virus reemerges with slightly slower kinetics [831].

A possible role of $U_{S} 11$ in viral dissemination may stem from its packing in extracellular vesicles (EVs). We have detected $\mathrm{U}_{\mathrm{S}} 11$ in $\mathrm{ESCRT}^{+} \mathrm{EV}_{\mathrm{s}}$ that have been isolated from cells infected with HSV-1 [225]. EVs that contain $\mathrm{U}_{\mathrm{S}} 11$ may reach neighboring uninfected cells and alter their status in order to regulate viral dissemination.

\section{Conclusions}

HSV-1 encodes a large set of genes that regulate different facets of the virus life cycle, such as virus entry, viral gene transcription and expression, DNA replication, virion formation and release, host evasion, and pathogenesis. Some of the non-essential proteins 
of HSV-1 have been studied more extensively than others, while the functions of some genes still remain unknown. Considering that the approximately 100 gene products encoded by the virus support both viral functions and host evasion simultaneously, it is mandatory that each gene product has multiple roles throughout the virus life cycle. This results in functional redundancy that contributes to the versatility of the virus. In this review, we summarized research that has been carried out on more than 50 non-essential proteins that function in different facets of the HSV-1 life cycle.

Non-essential genes are dispensable in vitro when ablated individually, and a lot of work to delineate their functions is based on deleting or mutating them and investigating their effects on viral processes and antiviral responses. However, many non-essential proteins work as components of functional networks in complex circuits, such as $\mathrm{U}_{\mathrm{L}} 11 / \mathrm{gE} / \mathrm{gD}$, $\mathrm{U}_{\mathrm{L}} 16 / \mathrm{gE} / \mathrm{U}_{\mathrm{L}} 11 / \mathrm{U}_{\mathrm{L}} 21 / \mathrm{VP} 22, \mathrm{U}_{\mathrm{L}} 31 / \mathrm{U}_{\mathrm{L}} 34 / \mathrm{U}_{\mathrm{S}} 3$, or ICP0/U $\mathrm{U}_{\mathrm{L}} 46 / \mathrm{U}_{\mathrm{L}} 47 / \mathrm{ICP} 22 / \mathrm{U}_{\mathrm{S}} 3 / \mathrm{U}_{\mathrm{L}} 13 /$ VP22 (described above). Therefore, it is likely that the field of HSV-1 research would benefit from investigating non-essential proteins not individually, but in tandem. An example is the work that has been done on HSV-1 mutants that trigger the formation of syncytia, in which regulatory interactions among HSV-1 proteins were described based on the effect of a non-essential protein deletion in the context of a syncytial HSV-1 mutation [672,709,832]. A potential problem that may arise by mutating non-essential genes in tandem is that the resulting virus may not be viable.

Another consideration for further research is the relevance of the model systems being used. Various non-essential proteins should be properly investigated in a relevant physiological context, depending on their function. For example, the role of non-essential proteins in cell-to-cell spread may give different insights when investigated in polarized epithelial cells, versus human fibroblasts, or the functions in trafficking will be different in neurons with long axons versus epithelial cells. Systems like organotypic brain slice cultures [833] can be used that recapitulate more closely the variable environment of a natural infection, considering that organotypic epithelial culture systems have been successfully utilized for the study of HSV-1 ribonucleotide reductase [834]. Such considerations extend to animal models as well, since the host immune regulation may be better investigated in a model other than mice. Spontaneous reactivation of latent HSV-1 occurs in humans and rabbits but not mice [835]. The human Stimulator of Interferon Genes (STING) is regulated in a different manner than the murine STING, complicating the study of non-essential protein functions on innate immunity $[836,837]$. It has also been described that ICP47 binding to the murine TAP is far weaker than the human one; therefore, the prevention of MHC I antigen presentation cannot be properly evaluated in mice, and this has implications for studies of neurovirulence and survival in the CNS [341,345,346]. Transgenic animal models, or alternative models, such as pigs, dogs, or monkeys [345], may have to be chosen carefully for research questions regarding the function of such non-essential proteins.

Another possibility for recapitulating a natural infection of the human CNS is the recently established brain organoids [838]. Recent advances in stem cell differentiation permit the use of human-induced pluripotent stem cells (hiPSCs) to generate three-dimensional (3D) neuron cultures, which are referred to as brain organoids. They exhibit neuronal heterogeneity and lamina-like structure [839]. Brain organoids can be infected with HSV-1 and exhibit inflammation, HSV-1 can establish hallmarks of latency in such cultures in the presence of antivirals like interferon (IFN), and HSV-1 can infect the outer laminar structure of these organoids moving further inside after infection [840,841]. All these elements make the use of brain organoids in HSV-1 research attractive. Investigation of latency can be complemented with the use of Lund human mesencephalic (LUHMES) cells [842]. These are human embryonic neuronal precursor cells that can be differentiated to postmitotic neurons and they can be used as a supplemental model to study latency and interactions of host and viral non-essential proteins. After infection of HSV-1, there is a loss of lytic gene transcription and an increase in the number of neurons that express latency-associated transcripts (LATs) [842]. The advantage of the latter system is that it does not require the presence of an antiviral factor like IFN $\beta$ to maintain latency. 
The non-essential genes of HSV-1 offer unique properties that can be utilized for the development of recombinant HSV-1 viruses suitable for oncolytic therapy [843]. The first oncolytic virus to receive regulatory approval in the United States was an attenuated HSV-1 (named T-VEC) that has been engineered to lack ICP34.5, a major HSV-1 neurovirulence factor, ICP47, which normally prevents MHC I antigen presentation, and to express the human GM-CSF gene, which promotes dendritic cell accumulation at sites of inflammation and enhances APC function [844]. T-VEC was approved for the treatment of patients with local unresectable malignant melanoma. T-VEC is currently being investigated for further potential uses against Merkel cell carcinoma, and other malignancies [845,846]. The multiple roles of HSV-1 non-essential proteins in vivo make the study of manipulating their function worthwhile for therapeutic purposes [847]. For example, engineered HSV-1 lacking the $\mathrm{U}_{\mathrm{L}} 39$ gene, which is required for replication in non-dividing cells (i.e., neurons), makes it an attractive candidate for targeting gliomas [848]. For similar reasons, HSV-1 mutants lacking the function of the uracil glycosylase $U_{L} 2$ [849] or $U_{L} 56$ are less neurovirulent and good candidates for further investigation. Besides oncolytic therapy, non-essential proteins can be utilized for vaccine research. An HSV-1 strain carrying a deletion in gK cannot infect neuronal axons and establish latency. This strain has been explored as a vaccine to confer protection against lethal intravaginal HSV-1 and HSV-2 challenge in mice and rhesus macaques $[850,851]$. ICP0-null or vhs-null viruses combined perhaps with other mutations could be explored for vaccine strategies due to their attenuated phenotype as well.

Significant research has been carried out on the roles of non-essential proteins of HSV-1 for many decades. While many aspects of the virus have been unveiled regarding the networks of interactions and the role of individual proteins in the virus life cycle, a lot remains to be clarified regarding the role of non-essential proteins in vivo. Further investigation of the non-essential HSV-1 proteins will allow us to better understand mechanisms of HSV-1 pathogenesis and disease, and will also enable effective harnessing of HSV-1 properties for cancer, gene therapy, and vaccine strategies.

Author Contributions: Conceptualization, M.K.; Writing-Original draft preparation, C.D., H.W. and M.K.; Review and editing, M.K.; Supervision, M.K.; Funding acquisition, M.K.; C.D. and H.W. have equally contributed to the manuscript in addition to the following information. All authors have read and agreed to the published version of the manuscript.

Funding: This research was funded by the NIAID R21AI144883 and the NIGMS P20GM113117 grants, and the KUMC enhancement award Y6K00080.

Conflicts of Interest: The authors declare no conflict of interest.

\section{References}

1. McGeoch, D.J.; Dolan, A.; Ralph, A.C. Toward a comprehensive phylogeny for mammalian and avian herpesviruses. J. Virol. 2000, 74, 10401-10406. [CrossRef] [PubMed]

2. Underdown, S.J.; Kumar, K.; Houldcroft, C. Network analysis of the hominin origin of Herpes Simplex virus 2 from fossil data. Virus Evol. 2017, 3. [CrossRef] [PubMed]

3. Knipe, D.M.; Howley, P. Fields Virology, 6th ed.; Lippincott Williams \& Wilkins: Philadelphia, PA, USA, 2013 ; Volume 1.

4. Mador, N.; Goldenberg, D.; Cohen, O.; Panet, A.; Steiner, I. Herpes simplex virus type 1 latency-associated transcripts suppress viral replication and reduce immediate-early gene mRNA levels in a neuronal cell line. J. Virol. 1998, 72, 5067-5075. [CrossRef] [PubMed]

5. Umbach, J.L.; Kramer, M.F.; Jurak, I.; Karnowski, H.W.; Coen, D.M.; Cullen, B.R. MicroRNAs expressed by herpes simplex virus 1 during latent infection regulate viral mRNAs. Nature 2008, 454, 780-783. [CrossRef] [PubMed]

6. Shen, W.; Sa e Silva, M.; Jaber, T.; Vitvitskaia, O.; Li, S.; Henderson, G.; Jones, C. Two small RNAs encoded within the first 1.5 kilobases of the herpes simplex virus type 1 latency-associated transcript can inhibit productive infection and cooperate to inhibit apoptosis. J. Virol. 2009, 83, 9131-9139. [CrossRef] [PubMed]

7. Croen, K.D.; Ostrove, J.M.; Dragovic, L.J.; Smialek, J.E.; Straus, S.E. Latent herpes simplex virus in human trigeminal ganglia. Detection of an immediate early gene "anti-sense" transcript by in situ hybridization. N. Engl. J. Med. 1987, 317, 1427-1432. [CrossRef] 
8. Mador, N.; Panet, A.; Latchman, D.; Steiner, I. Expression and splicing of the latency-associated transcripts of herpes simplex virus type 1 in neuronal and non-neuronal cell lines. J. Biochem. 1995, 117, 1288-1297. [CrossRef]

9. Garber, D.A.; Schaffer, P.A.; Knipe, D.M. A LAT-associated function reduces productive-cycle gene expression during acute infection of murine sensory neurons with herpes simplex virus type 1. J. Virol. 1997, 71, 5885-5893. [CrossRef]

10. Chen, S.H.; Kramer, M.F.; Schaffer, P.A.; Coen, D.M. A viral function represses accumulation of transcripts from productive-cycle genes in mouse ganglia latently infected with herpes simplex virus. J. Virol. 1997, 71, 5878-5884. [CrossRef]

11. Wagner, E.K.; Bloom, D.C. Experimental investigation of herpes simplex virus latency. Clin. Microbiol. Rev. 1997, 10, 419-443. [CrossRef]

12. Ahmed, M.; Lock, M.; Miller, C.G.; Fraser, N.W. Regions of the herpes simplex virus type 1 latency-associated transcript that protect cells from apoptosis in vitro and protect neuronal cells in vivo. J. Virol. 2002, 76, 717-729. [CrossRef] [PubMed]

13. Henderson, G.; Peng, W.; Jin, L.; Perng, G.-C.; Nesburn, A.B.; Wechsler, S.L.; Jones, C. Regulation of caspase 8- and caspase 9-induced apoptosis by the herpes simplex virus type 1 latency-associated transcript. J. Neurovirol. 2002, 8, 103-111. [CrossRef] [PubMed]

14. Inman, M.; Perng, G.-C.; Henderson, G.; Ghiasi, H.; Nesburn, A.B.; Wechsler, S.L.; Jones, C. Region of herpes simplex virus type 1 latency-associated transcript sufficient for wild-type spontaneous reactivation promotes cell survival in tissue culture. J. Virol. 2001, 75, 3636-3646. [CrossRef] [PubMed]

15. Jin, L.; Peng, W.; Perng, G.-C.; Brick, D.J.; Nesburn, A.B.; Jones, C.; Wechsler, S.L. Identification of herpes simplex virus type 1 latency-associated transcript sequences that both inhibit apoptosis and enhance the spontaneous reactivation phenotype. J. Virol. 2003, 77, 6556-6561. [CrossRef] [PubMed]

16. Perng, G.C.; Ghiasi, H.; Slanina, S.M.; Nesburn, A.B.; Wechsler, S.L. The spontaneous reactivation function of the herpes simplex virus type 1 LAT gene resides completely within the first 1.5 kilobases of the 8.3-kilobase primary transcript. J. Virol. 1996, 70, 976-984. [CrossRef] [PubMed]

17. Peng, W.; Henderson, G.; Perng, G.-C.; Nesburn, A.B.; Wechsler, S.L.; Jones, C. The gene that encodes the herpes simplex virus type 1 latency-associated transcript influences the accumulation of transcripts $\left(\mathrm{Bcl}-\mathrm{x}_{\mathrm{L}}\right.$ and $\left.\mathrm{Bcl}-\mathrm{x}_{\mathrm{S}}\right)$ that encode apoptotic regulatory proteins. J. Virol. 2003, 77, 10714-10718. [CrossRef]

18. Perng, G.-C.; Jones, C.; Ciacci-Zanella, J.; Stone, M.; Henderson, G.; Yukht, A.; Slanina, S.M.; Hofman, F.M.; Ghiasi, H.; Nesburn, A.B.; et al. Virus-induced neuronal apoptosis blocked by the herpes simplex virus latency-associated transcript. Science 2000, 287, 1500-1503. [CrossRef]

19. Honess, R.W.; Roizman, B. Regulation of herpesvirus macromolecular synthesis. I. Cascade regulation of the synthesis of three groups of viral proteins. J. Virol. 1974, 14, 8-19. [CrossRef]

20. Honess, R.W.; Roizman, B. Regulation of herpesvirus macromolecular synthesis: Sequential transition of polypeptide synthesis requires functional viral polypeptides. Proc. Natl. Acad. Sci. USA 1975, 72, 1276-1280. [CrossRef]

21. Longnecker, R.; Roizman, B. Clustering of genes dispensable for growth in culture in the S component of the HSV-1 genome. Science 1987, 236, 573-576. [CrossRef]

22. McGeoch, D.J.; Dolan, A.; Frame, M.C. DNA sequence of the region in the genome of herpes simplex virus type 1 containing the exonuclease gene and neighbouring genes. Nucleic Acids Res. 1986, 14, 3435-3448. [CrossRef] [PubMed]

23. Toh, Y.; Tanaka, S.; Liu, Y.; Hidaka, Y.; Mori, R. Molecular characterization of naturally occuring glycoprotein C-negative herpes simplex virus type 1. Arch. Virol. 1993, 129, 119-130. [CrossRef] [PubMed]

24. Hidaka, Y.; Sakuma, S.; Kumano, Y.; Minagawa, H.; Mori, R. Characterization of glycoprotein C-negative mutants of herpes simplex virus type 1 isolated from a patient with keratitis. Arch. Virol. 1990, 113, 195-207. [CrossRef]

25. Kalamvoki, M.; Roizman, B. HSV-1 degrades, stabilizes, requires, or is stung by STING depending on ICP0, the US3 protein kinase, and cell derivation. Proc. Natl. Acad. Sci. USA 2014, 111, E611-7. [CrossRef] [PubMed]

26. Deschamps, T.; Kalamvoki, M. Impaired STING pathway in human osteosarcoma U2OS cells contributes to the growth of ICP0-null mutant herpes simplex virus. J. Virol. 2017, 91. [CrossRef]

27. Ackermann, M.; Braun, D.K.; Pereira, L.; Roizman, B. Characterization of herpes simplex virus 1 alpha proteins 0,4 , and 27 with monoclonal antibodies. J. Virol. 1984, 52, 108-118. [CrossRef]

28. Stow, N.D.; Stow, E.C. Isolation and characterization of a herpes simplex virus type 1 mutant containing a deletion within the gene encoding the immediate early polypeptide Vmw110. J. Gen. Virol. 1986, 67, 2571-2585. [CrossRef]

29. Sacks, W.R.; Schaffer, P.A. Deletion mutants in the gene encoding the herpes simplex virus type 1 immediate-early protein ICP0 exhibit impaired growth in cell culture. J. Virol. 1987, 61, 829-839. [CrossRef]

30. Yao, F.; Schaffer, P.A. An activity specified by the osteosarcoma line U2OS can substitute functionally for ICP0, a major regulatory protein of herpes simplex virus type 1. J. Virol. 1995, 69, 6249-6258. [CrossRef]

31. Chen, J.; Silverstein, S. Herpes simplex viruses with mutations in the gene encoding ICP0 are defective in gene expression. J. Virol. 1992, 66, 2916-2927. [CrossRef]

32. Everett, R.D.; Boutell, C.; Orr, A. Phenotype of a herpes simplex virus type 1 mutant that fails to express immediate-early regulatory protein ICP0. J. Virol. 2004, 78, 1763-1774. [CrossRef] [PubMed]

33. Kalamvoki, M.; Roizman, B. Role of herpes simplex virus ICP0 in the transactivation of genes introduced by infection or transfection: A reappraisal. J. Virol. 2010, 84, 4222-4228. [CrossRef] [PubMed] 
34. Alandijany, T.; Roberts, A.P.E.; Conn, K.L.; Loney, C.; McFarlane, S.; Orr, A.; Boutell, C. Distinct temporal roles for the promyelocytic leukaemia (PML) protein in the sequential regulation of intracellular host immunity to HSV-1 infection. PLoS Pathog. 2018, 14, e1006927. [CrossRef]

35. Sandri-Goldin, R.M.; Sekulovich, R.E.; Leary, K. The alpha protein ICP0 does not appear to play a major role in the regulation of herpes simplex virus gene expression during infection in tissue culture. Nucleic Acids Res. 1987, 15, 905-919. [CrossRef] [PubMed]

36. Moriuchi, H.; Moriuchi, M.; Straus, S.E.; Cohen, J.I. Varicella-zoster virus (VZV) open reading frame 61 protein transactivates VZV gene promoters and enhances the infectivity of VZV DNA. J. Virol. 1993, 67, 4290-4295. [CrossRef] [PubMed]

37. Everett, R.; Orr, A.; Elliott, M. The equine herpesvirus 1 gene 63 RING finger protein partially complements Vmw110, its herpes simplex virus type 1 counterpart. J. Gen. Virol. 1995, 76, 2369-2374. [CrossRef] [PubMed]

38. Everett, R.D.; Boutell, C.; McNair, C.; Grant, L.; Orr, A. Comparison of the biological and biochemical activities of several members of the alphaherpesvirus ICP0 family of proteins. J. Virol. 2010, 84, 3476-3487. [CrossRef]

39. Quinlan, M.P.; Knipe, D.M. Stimulation of expression of a herpes simplex virus DNA-binding protein by two viral functions. Mol. Cell. Biol. 1985, 5, 957-963. [CrossRef]

40. Knipe, D.M.; Smith, J.L. A mutant herpesvirus protein leads to a block in nuclear localization of other viral proteins. Mol. Cell. Biol. 1986, 6, 2371-2381. [CrossRef]

41. Gelman, I.H.; Silverstein, S. Identification of immediate early genes from herpes simplex virus that transactivate the virus thymidine kinase gene. Proc. Natl. Acad. Sci. USA 1985, 82, 5265-5269. [CrossRef]

42. Mosca, J.D.; Bednarik, D.P.; Raj, N.B.; Rosen, C.A.; Sodroski, J.G.; Haseltine, W.A.; Hayward, G.S.; Pitha, P.M. Activation of human immunodeficiency virus by herpesvirus infection: Identification of a region within the long terminal repeat that responds to a trans-acting factor encoded by herpes simplex virus 1. Proc. Natl. Acad. Sci. USA 1987, 84, 7408-7412. [CrossRef] [PubMed]

43. Ostrove, J.M.; Leonard, J.; Weck, K.E.; Rabson, A.B.; Gendelman, H.E. Activation of the human immunodeficiency virus by herpes simplex virus type 1. J. Virol. 1987, 61, 3726-3732. [CrossRef] [PubMed]

44. Goldstein, D.J.; Weller, S.K. Herpes simplex virus type 1-induced ribonucleotide reductase activity is dispensable for virus growth and DNA synthesis: Isolation and characterization of an ICP6 lacZ insertion mutant. J. Virol. 1988, 62, 196-205. [CrossRef] [PubMed]

45. McCusker, C.T.; Bacchetti, S. The responsiveness of human papillomavirus upstream regulatory regions to herpes simplex virus immediate early proteins. Virus Res. 1988, 11, 199-207. [CrossRef]

46. Gius, D.; Laimins, L.A. Activation of human papillomavirus type 18 gene expression by herpes simplex virus type 1 viral transactivators and a phorbol ester. J. Virol. 1989, 63, 555-563. [CrossRef] [PubMed]

47. Margolis, D.M.; Rabson, A.B.; Straus, S.E.; Ostrove, J.M. Transactivation of the HIV-1 LTR by HSV-1 immediate-early genes. Virology 1992, 186, 788-791. [CrossRef]

48. Nabel, G.J.; Rice, S.A.; Knipe, D.M.; Baltimore, D. Alternative mechanisms for activation of human immunodeficiency virus enhancer in T cells. Science 1988, 239, 1299-1302. [CrossRef]

49. Nieto-Torres, J.L.; DeDiego, M.L.; Verdiá-Báguena, C.; Jimenez-Guardeño, J.M.; Regla-Nava, J.A.; Fernandez-Delgado, R.; Castaño-Rodriguez, C.; Alcaraz, A.; Torres, J.; Aguilella, V.M.; et al. Severe acute respiratory syndrome coronavirus envelope protein ion channel activity promotes virus fitness and pathogenesis. PLoS Pathog. 2014, 10, e1004077. [CrossRef]

50. O'Hare, P.; Hayward, G.S. Three trans-acting regulatory proteins of herpes simplex virus modulate immediate-early gene expression in a pathway involving positive and negative feedback regulation. J. Virol. 1985, 56, 723-733. [CrossRef]

51. Cai, W.; Schaffer, P.A. Herpes simplex virus type 1 ICP0 regulates expression of immediate-early, early, and late genes in productively infected cells. J. Virol. 1992, 66, 2904-2915. [CrossRef]

52. Boutell, C.; Sadis, S.; Everett, R.D. Herpes simplex virus type 1 immediate-early protein ICP0 and is isolated RING finger domain act as ubiquitin E3 ligases in vitro. J. Virol. 2002, 76, 841-850. [CrossRef] [PubMed]

53. Everett, R.D.; Barlow, P.; Milner, A.; Luisi, B.; Orr, A.; Hope, G.; Lyon, D. A novel arrangement of zinc-binding residues and secondary structure in the C3HC4 motif of an alpha herpes virus protein family. J. Mol. Biol. 1993, 234, 1038-1047. [CrossRef] [PubMed]

54. Grant, K.; Grant, L.; Tong, L.; Boutell, C. Depletion of intracellular zinc inhibits the ubiquitin ligase activity of viral regulatory protein ICP0 and restricts herpes simplex virus 1 replication in cell culture. J. Virol. 2012, 86, 4029-4033. [CrossRef] [PubMed]

55. Lium, E.K.; Silverstein, S. Mutational analysis of the herpes simplex virus type 1 ICP0 C3HC4 zinc ring finger reveals a requirement for ICP0 in the expression of the essential alpha27 gene. J. Virol. 1997, 71, 8602-8614. [CrossRef]

56. Boutell, C.; Orr, A.; Everett, R.D. PML residue lysine 160 is required for the degradation of PML induced by herpes simplex virus type 1 regulatory protein ICP0. J. Virol. 2003, 77, 8686-8694. [CrossRef]

57. Boutell, C.; Everett, R.D. The herpes simplex virus type 1 (HSV-1) regulatory protein ICP0 interacts with and Ubiquitinates p53. J. Biol. Chem. 2003, 278, 36596-36602. [CrossRef]

58. Boutell, C.; Canning, M.; Orr, A.; Everett, R.D. Reciprocal activities between herpes simplex virus type 1 regulatory protein ICP0, a ubiquitin E3 ligase, and ubiquitin-specific protease USP7. J. Virol. 2005, 79, 12342-12354. [CrossRef]

59. Gu, H.; Roizman, B. The degradation of promyelocytic leukemia and Sp100 proteins by herpes simplex virus 1 is mediated by the ubiquitin-conjugating enzyme UbcH5a. Proc. Natl. Acad. Sci. USA 2003, 100, 8963-8968. [CrossRef] 
60. Vanni, E.; Gatherer, D.; Tong, L.; Everett, R.D.; Boutell, C. Functional characterization of residues required for the herpes simplex virus $1 \mathrm{E} 3$ ubiquitin ligase ICP0 to interact with the cellular E2 ubiquitin-conjugating enzyme UBE2D1 (UbcH5a). J. Virol. 2012, 86, 6323-6333. [CrossRef]

61. Maul, G.G.; Guldner, H.H.; Spivack, J.G. Modification of discrete nuclear domains induced by herpes simplex virus type 1 immediate early gene 1 product (ICP0). J. Gen. Virol. 1993, 74, 2679-2690. [CrossRef]

62. Mullen, M.A.; Ciufo, D.M.; Hayward, G.S. Mapping of intracellular localization domains and evidence for colocalization interactions between the IE110 and IE175 nuclear transactivator proteins of herpes simplex virus. J. Virol. 1994, 68, 3250-3266. [CrossRef] [PubMed]

63. Everett, R.D.; Maul, G.G. HSV-1 IE protein Vmw110 causes redistribution of PML. EMBO J. 1994, 13, 5062-5069. [CrossRef] [PubMed]

64. Everett, R.D.; Murray, J.; Orr, A.; Preston, C.M. Herpes simplex virus type 1 genomes are associated with ND10 nuclear substructures in quiescently infected human fibroblasts. J. Virol. 2007, 81, 10991-11004. [CrossRef] [PubMed]

65. Cohen, C.; Corpet, A.; Roubille, S.; Maroui, M.A.; Poccardi, N.; Rousseau, A.; Kleijwegt, C.; Binda, O.; Texier, P.; Sawtell, N.; et al. Promyelocytic leukemia (PML) nuclear bodies (NBs) induce latent/quiescent HSV-1 genomes chromatinization through a PML NB/Histone H3.3/H3.3 Chaperone Axis. PLoS Pathog. 2018, 14, e1007313. [CrossRef]

66. Maroui, M.A.; Callé, A.; Cohen, C.; Streichenberger, N.; Texier, P.; Takissian, J.; Rousseau, A.; Poccardi, N.; Welsch, J.; Corpet, A.; et al. Latency entry of herpes simplex Virus 1 is determined by the interaction of its genome with the nuclear environment. PLoS Pathog. 2016, 12, e1005834. [CrossRef]

67. Thompson, R.L.; Sawtell, N.M. Evidence that the herpes simplex virus type 1 ICP0 protein does not initiate reactivation from latency in vivo. J. Virol. 2006, 80, 10919-10930. [CrossRef]

68. Cai, W.; Astor, T.L.; Liptak, L.M.; Cho, C.; Coen, D.M.; Schaffer, P.A. The herpes simplex virus type 1 regulatory protein ICP0 enhances virus replication during acute infection and reactivation from latency. J. Virol. 1993, 67, 7501-7512. [CrossRef]

69. Roizman, B. The checkpoints of viral gene expression in productive and latent infection: The role of the HDAC/CoREST/LSD1/ REST repressor complex. J. Virol. 2011, 85, 7474-7482. [CrossRef]

70. Härle, P.; Sainz, B.J.; Carr, D.J.J.; Halford, W.P. The immediate-early protein, ICP0, is essential for the resistance of herpes simplex virus to interferon-alpha/beta. Virology 2002, 293, 295-304. [CrossRef]

71. Halford, W.P.; Schaffer, P.A. ICP0 is required for efficient reactivation of herpes simplex virus type 1 from neuronal latency. J. Virol. 2001, 75, 3240-3249. [CrossRef]

72. Deschamps, T.; Waisner, H.; Dogrammatzis, C.; Roy, A.; Chacko, S.; Perera, C.; Prisinzano, T.E.; Kalamvoki, M. Discovery of small-molecule inhibitors targeting the E3 ubiquitin ligase activity of the herpes simplex virus 1 ICP0 protein using an in vitro high-throughput screening assay. J. Virol. 2019, 93. [CrossRef] [PubMed]

73. Hagglund, R.; Roizman, B. Herpes simplex virus 1 mutant in which the ICP0 HUL-1 E3 ubiquitin ligase site is disrupted stabilizes cdc34 but degrades D-type cyclins and exhibits diminished neurotoxicity. J. Virol. 2003, 77, 13194-13202. [CrossRef] [PubMed]

74. Gu, H.; Poon, A.P.; Roizman, B. During its nuclear phase the multifunctional regulatory protein ICP0 undergoes proteolytic cleavage characteristic of polyproteins. Proc. Natl. Acad. Sci. USA 2009, 106, 19132-19137. [CrossRef]

75. Boutell, C.; Cuchet-Lourenço, D.; Vanni, E.; Orr, A.; Glass, M.; McFarlane, S.; Everett, R.D. A viral ubiquitin ligase has substrate preferential SUMO targeted ubiquitin ligase activity that counteracts intrinsic antiviral defence. PLoS Pathog. 2011, 7, e1002245. [CrossRef]

76. Cuchet-Lourenço, D.; Vanni, E.; Glass, M.; Orr, A.; Everett, R.D. Herpes simplex virus 1 ubiquitin ligase ICP0 interacts with PML isoform I and induces its SUMO-independent degradation. J. Virol. 2012, 86, 11209-11222. [CrossRef]

77. Sloan, E.; Tatham, M.H.; Groslambert, M.; Glass, M.; Orr, A.; Hay, R.T.; Everett, R.D. Analysis of the SUMO2 proteome during HSV-1 infection. PLoS Pathog. 2015, 11, e1005059. [CrossRef]

78. Everett, R.D.; Boutell, C.; Pheasant, K.; Cuchet-Lourenço, D.; Orr, A. Sequences related to SUMO interaction motifs in herpes simplex virus 1 protein ICP0 act cooperatively to stimulate virus infection. J. Virol. 2014, 88, 2763-2774. [CrossRef] [PubMed]

79. Everett, R.D.; Parsy, M.-L.; Orr, A. Analysis of the functions of herpes simplex virus type 1 regulatory protein ICP0 that are critical for lytic infection and derepression of quiescent viral genomes. J. Virol. 2009, 83, 4963-4977. [CrossRef] [PubMed]

80. Everett, R.D.; Freemont, P.; Saitoh, H.; Dasso, M.; Orr, A.; Kathoria, M.; Parkinson, J. The disruption of ND10 during herpes simplex virus infection correlates with the Vmw110- and proteasome-dependent loss of several PML isoforms. J. Virol. 1998, 72, 6581-6591. [CrossRef] [PubMed]

81. Chelbi-Alix, M.K.; de Thé, H. Herpes virus induced proteasome-dependent degradation of the nuclear bodies-associated PML and Sp100 proteins. Oncogene 1999, 18, 935-941. [CrossRef] [PubMed]

82. Sha, Z.; Blyszcz, T.; González-Prieto, R.; Vertegaal, A.C.O.; Goldberg, A.L. Inhibiting ubiquitination causes an accumulation of SUMOylated newly synthesized nuclear proteins at PML bodies. J. Biol. Chem. 2019, 294, 15218-15234. [CrossRef] [PubMed]

83. Everett, R.D.; Murray, J. ND10 components relocate to sites associated with herpes simplex virus type 1 nucleoprotein complexes during virus infection. J. Virol. 2005, 79, 5078-5089. [CrossRef]

84. Maroui, M.A.; Maarifi, G.; McManus, F.P.; Lamoliatte, F.; Thibault, P.; Chelbi-Alix, M.K. Promyelocytic Leukemia Protein (PML) requirement for interferon-induced global cellular SUMOylation. Mol. Cell. Proteom. 2018, 17, 1196-1208. [CrossRef] [PubMed]

85. Sloan, E.; Orr, A.; Everett, R.D. MORC3, a Component of PML nuclear bodies, has a role in restricting herpes simplex virus 1 and human cytomegalovirus. J. Virol. 2016, 90, 8621-8633. [CrossRef] [PubMed] 
86. Conn, K.L.; Wasson, P.; McFarlane, S.; Tong, L.; Brown, J.R.; Grant, K.G.; Domingues, P.; Boutell, C. Novel role for protein inhibitor of activated STAT 4 (PIAS4) in the restriction of herpes simplex virus 1 by the cellular intrinsic antiviral immune response. J. Virol. 2016, 90, 4807-4826. [CrossRef]

87. Brown, J.R.; Conn, K.L.; Wasson, P.; Charman, M.; Tong, L.; Grant, K.; McFarlane, S.; Boutell, C. SUMO ligase protein inhibitor of activated STAT1 (PIAS1) is a constituent promyelocytic leukemia nuclear body protein that contributes to the intrinsic antiviral immune response to herpes simplex virus 1. J. Virol. 2016, 90, 5939-5952. [CrossRef]

88. Lees-Miller, S.P.; Long, M.C.; Kilvert, M.A.; Lam, V.; Rice, S.A.; Spencer, C.A. Attenuation of DNA-dependent protein kinase activity and its catalytic subunit by the herpes simplex virus type 1 transactivator ICP0. J. Virol. 1996, 70, 7471-7477. [CrossRef]

89. Parkinson, J.; Lees-Miller, S.P.; Everett, R.D. Herpes simplex virus type 1 immediate-early protein vmw 110 induces the proteasomedependent degradation of the catalytic subunit of DNA-dependent protein kinase. J. Virol. 1999, 73, 650-657. [CrossRef]

90. Jackson, S.A.; DeLuca, N.A. Relationship of herpes simplex virus genome configuration to productive and persistent infections. Proc. Natl. Acad. Sci. USA 2003, 100, 7871-7876. [CrossRef]

91. Lilley, C.E.; Chaurushiya, M.S.; Boutell, C.; Landry, S.; Suh, J.; Panier, S.; Everett, R.D.; Stewart, G.S.; Durocher, D.; Weitzman, M.D. A viral E3 ligase targets RNF8 and RNF168 to control histone ubiquitination and DNA damage responses. EMBO J. 2010, 29, 943-955. [CrossRef]

92. Lilley, C.E.; Chaurushiya, M.S.; Boutell, C.; Everett, R.D.; Weitzman, M.D. The intrinsic antiviral defense to incoming HSV-1 genomes includes specific DNA repair proteins and is counteracted by the viral protein ICP0. PLoS Pathog. 2011, 7, e1002084. [CrossRef]

93. Chaurushiya, M.S.; Lilley, C.E.; Aslanian, A.; Meisenhelder, J.; Scott, D.C.; Landry, S.; Ticau, S.; Boutell, C.; Yates, J.R., III Schulman, B.A.; et al. Viral E3 ubiquitin ligase-mediated degradation of a cellular E3: Viral mimicry of a cellular phosphorylation mark targets the RNF8 FHA domain. Mol. Cell 2012, 46, 79-90. [CrossRef] [PubMed]

94. Nagel, C.-H.; Albrecht, N.; Milovic-Holm, K.; Mariyanna, L.; Keyser, B.; Abel, B.; Weseloh, B.; Hofmann, T.G.; Eibl, M.M.; Hauber, J. Herpes simplex virus immediate-early protein ICP0 is targeted by SIAH-1 for proteasomal degradation. J. Virol. 2011, 85, 7644-7657. [CrossRef] [PubMed]

95. Conwell, S.E.; White, A.E.; Harper, J.W.; Knipe, D.M. Identification of TRIM27 as a novel degradation target of herpes simplex virus 1 ICP0. J. Virol. 2015, 89, 220-229. [CrossRef] [PubMed]

96. Deshmane, S.L.; Fraser, N.W. During latency, herpes simplex virus type 1 DNA is associated with nucleosomes in a chromatin structure. J. Virol. 1989, 63, 943-947. [CrossRef]

97. Herrera, F.J.; Triezenberg, S.J. VP16-dependent association of chromatin-modifying coactivators and underrepresentation of histones at immediate-early gene promoters during herpes simplex virus infection. J. Virol. 2004, 78, 9689-9696. [CrossRef]

98. Kent, J.R.; Zeng, P.-Y.; Atanasiu, D.; Gardner, J.; Fraser, N.W.; Berger, S.L. During lytic infection herpes simplex virus type 1 is associated with histones bearing modifications that correlate with active transcription. J. Virol. 2004, 78, 10178-10186. [CrossRef]

99. Huang, J.; Kent, J.R.; Placek, B.; Whelan, K.A.; Hollow, C.M.; Zeng, P.-Y.; Fraser, N.W.; Berger, S.L. Trimethylation of histone H3 lysine 4 by Set1 in the lytic infection of human herpes simplex virus 1. J. Virol. 2006, 80, 5740-5746. [CrossRef]

100. Cabral, J.M.; Oh, H.S.; Knipe, D.M. ATRX promotes maintenance of herpes simplex virus heterochromatin during chromatin stress. eLife 2018, 7. [CrossRef]

101. Lee, J.S.; Raja, P.; Knipe, D.M. Herpesviral ICP0 protein promotes two waves of heterochromatin removal on an early viral promoter during lytic infection. mBio 2016, 7. [CrossRef]

102. Lomonte, P.; Thomas, J.; Texier, P.; Caron, C.; Khochbin, S.; Epstein, A.L. Functional interaction between class II histone deacetylases and ICP0 of herpes simplex virus type 1. J. Virol. 2004, 78, 6744-6757. [CrossRef] [PubMed]

103. Poon, A.P.W.; Gu, H.; Roizman, B. ICP0 and the $\mathrm{U}_{\mathrm{S}} 3$ protein kinase of herpes simplex virus 1 independently block histone deacetylation to enable gene expression. Proc. Natl. Acad. Sci. USA 2006, 103, 9993-9998. [CrossRef] [PubMed]

104. Hobbs, W.E., II; DeLuca, N.A. Perturbation of cell cycle progression and cellular gene expression as a function of herpes simplex virus ICP0. J. Virol. 1999, 73, 8245-8255. [CrossRef] [PubMed]

105. Poon, A.P.W.; Liang, Y.; Roizman, B. Herpes simplex virus 1 gene expression is accelerated by inhibitors of histone deacetylases in rabbit skin cells infected with a mutant carrying a cDNA copy of the infected-cell protein no. 0. J. Virol. 2003, 77, 12671-12678. [CrossRef] [PubMed]

106. Kalamvoki, M.; Roizman, B. Circadian CLOCK histone acetyl transferase localizes at ND10 nuclear bodies and enables herpes simplex virus gene expression. Proc. Natl. Acad. Sci. USA 2010, 107, 17721-17726. [CrossRef] [PubMed]

107. Kalamvoki, M.; Roizman, B. The Histone Acetyltransferase CLOCK is an essential component of the herpes simplex virus 1 transcriptome that includes TFIID, ICP4, ICP27, and ICP22. J. Virol. 2011, 85, 9472-9477. [CrossRef]

108. Kalamvoki, M.; Roizman, B. Interwoven roles of cyclin D3 and cdk4 recruited by ICP0 and ICP4 in the expression of herpes simplex virus genes. J. Virol. 2010, 84, 9709-9717. [CrossRef]

109. Cliffe, A.R.; Knipe, D.M. Herpes simplex virus ICP0 promotes both histone removal and acetylation on viral DNA during lytic infection. J. Virol. 2008, 82, 12030-12038. [CrossRef]

110. Kutluay, S.B.; Triezenberg, S.J. Regulation of histone deposition on the herpes simplex virus type 1 genome during lytic infection. J. Virol. 2009, 83, 5835-5845. [CrossRef] [PubMed]

111. Placek, B.J.; Huang, J.; Kent, J.R.; Dorsey, J.; Rice, L.; Fraser, N.W.; Berger, S.L. The histone variant H3.3 regulates gene expression during lytic infection with herpes simplex virus type 1. J. Virol. 2009, 83, 1416-1421. [CrossRef] 
112. Gu, H.; Roizman, B. Engagement of the lysine-specific demethylase/HDAC1/CoREST/REST complex by herpes simplex virus 1. J. Virol. 2009, 83, 4376-4385. [CrossRef] [PubMed]

113. Gu, H.; Liang, Y.; Mandel, G.; Roizman, B. Components of the REST/CoREST/histone deacetylase repressor complex are disrupted, modified, and translocated in HSV-1-infected cells. Proc. Natl. Acad. Sci. USA 2005, 102, 7571-7576. [CrossRef] [PubMed]

114. Gu, H.; Roizman, B. Herpes simplex virus-infected cell protein 0 blocks the silencing of viral DNA by dissociating histone deacetylases from the CoREST-REST complex. Proc. Natl. Acad. Sci. USA 2007, 104, 17134-17139. [CrossRef] [PubMed]

115. Kalamvoki, M.; Roizman, B. Nuclear retention of ICP0 in cells exposed to HDAC inhibitor or transfected with DNA before infection with herpes simplex virus 1. Proc. Natl. Acad. Sci. USA 2008, 105, 20488-20493. [CrossRef]

116. Merkl, P.E.; Orzalli, M.H.; Knipe, D.M. Mechanisms of host IFI16, PML, and Daxx protein restriction of herpes simplex virus 1 replication. J. Virol. 2018, 92. [CrossRef]

117. Lukashchuk, V.; Everett, R.D. Regulation of ICP0-null mutant herpes simplex virus type 1 infection by ND10 components ATRX and hDaxx. J. Virol. 2010, 84, 4026-4040. [CrossRef]

118. Everett, R.D. Dynamic response of IFI16 and promyelocytic leukemia nuclear body components to herpes simplex virus 1 infection. J. Virol. 2016, 90, 167-179. [CrossRef]

119. Diner, B.A.; Lum, K.K.; Toettcher, J.E.; Cristea, I.M. Viral DNA sensors IFI16 and cyclic GMP-AMP synthase possess distinct functions in regulating viral gene expression, immune defenses, and apoptotic responses during herpesvirus infection. $m B i o$ 2016, 7. [CrossRef]

120. McFarlane, S.; Orr, A.; Roberts, A.P.E.; Conn, K.L.; Iliev, V.; Loney, C.; Filipe, A.d.S.; Smollett, K.; Gu, Q.; Robertson, N.; et al. The histone chaperone HIRA promotes the induction of host innate immune defences in response to HSV-1 infection. PLoS Pathog. 2019, 15, e1007667. [CrossRef]

121. Jurak, I.; Silverstein, L.B.; Sharma, M.; Coen, D.M. Herpes simplex virus is equipped with RNA- and protein-based mechanisms to repress expression of ATRX, an effector of intrinsic immunity. J. Virol. 2012, 86, 10093-10102. [CrossRef]

122. Orzalli, M.H.; DeLuca, N.A.; Knipe, D.M. Nuclear IFI16 induction of IRF-3 signaling during herpesviral infection and degradation of IFI16 by the viral ICP0 protein. Proc. Natl. Acad. Sci. USA 2012, 109. [CrossRef] [PubMed]

123. Orzalli, M.H.; Broekema, N.M.; Knipe, D.M. Relative contributions of herpes simplex virus 1 ICP0 and vhs to loss of cellular IFI16 vary in different human cell types. J. Virol. 2016, 90, 8351-8359. [CrossRef] [PubMed]

124. Cuchet-Lourenço, D.; Anderson, G.; Sloan, E.; Orr, A.; Everett, R.D. The viral ubiquitin ligase ICP0 is neither sufficient nor necessary for degradation of the cellular DNA sensor IFI16 during herpes simplex virus 1 infection. J. Virol. 2013, 87, 13422-13432. [CrossRef] [PubMed]

125. Diner, B.A.; Lum, K.K.; Javitt, A.; Cristea, I.M. Interactions of the antiviral factor interferon gamma-inducible protein 16 (IFI16) mediate immune signaling and herpes simplex virus-1 immunosuppression. Mol. Cell. Proteomics 2015, 14, 2341-2356. [CrossRef]

126. Kawaguchi, Y.; van Sant, C.; Roizman, B. Herpes simplex virus 1 alpha regulatory protein ICP0 interacts with and stabilizes the cell cycle regulator cyclin D3. J. Virol. 1997, 71, 7328-7336. [CrossRef]

127. Kalamvoki, M.; Roizman, B. ICP0 enables and monitors the function of D cyclins in herpes simplex virus 1 infected cells. Proc. Natl. Acad. Sci. USA 2009, 106, 14576-14580. [CrossRef]

128. Li, H.; Baskaran, R.; Krisky, D.M.; Bein, K.; Grandi, P.; Cohen, J.B.; Glorioso, J.C. Chk2 is required for HSV-1 ICP0-mediated G2/M arrest and enhancement of virus growth. Virology 2008, 375, 13-23. [CrossRef]

129. Lomonte, P.; Sullivan, K.F.; Everett, R.D. Degradation of nucleosome-associated centromeric histone H3-like protein CENP-A induced by herpes simplex virus type 1 protein ICP0. J. Biol. Chem. 2001, 276, 5829-5835. [CrossRef]

130. Lomonte, P.; Morency, E. Centromeric protein CENP-B proteasomal degradation induced by the viral protein ICP0. FEBS Lett. 2007, 581, 658-662. [CrossRef]

131. Everett, R.D.; Earnshaw, W.C.; Findlay, J.; Lomonte, P. Specific destruction of kinetochore protein CENP-C and disruption of cell division by herpes simplex virus immediate-early protein Vmw110. EMBO J. 1999, 18, 1526-1538. [CrossRef]

132. Gross, S.; Catez, F.; Masumoto, H.; Lomonte, P. Centromere architecture breakdown induced by the viral E3 ubiquitin ligase ICP0 protein of herpes simplex virus type 1. PLOS ONE 2012, 7, e44227. [CrossRef] [PubMed]

133. Deng, Z.; Kim, E.T.; Vladimirova, O.; Dheekollu, J.; Wang, Z.; Newhart, A.; Liu, D.; Myers, J.L.; Hensley, S.E.; Moffat, J.; et al. HSV-1 remodels host telomeres to facilitate viral replication. Cell Rep. 2014, 9, 2263-2278. [CrossRef] [PubMed]

134. Mossman, K.L.; Saffran, H.A.; Smiley, J.R. Herpes simplex virus ICP0 mutants are hypersensitive to interferon. J. Virol. 2000, 74, 2052-2056. [CrossRef] [PubMed]

135. Leib, D.A.; Harrison, T.E.; Laslo, K.M.; Machalek, M.A.; Moorman, N.J.; Virgin, H.W. Interferons regulate the phenotype of wild-type and mutant herpes simplex viruses in vivo. J. Exp. Med. 1999, 189, 663-672. [CrossRef] [PubMed]

136. Burleigh, K.; Maltbaek, J.H.; Cambier, S.; Green, R.; Gale, M.J.; James, R.C.; Stetson, D.B. Human DNA-PK activates a STINGindependent DNA sensing pathway. Sci. Immunol. 2020, 5. [CrossRef] [PubMed]

137. Orzalli, M.H.; Broekema, N.M.; Diner, B.A.; Hancks, D.C.; Elde, N.C.; Cristea, I.M.; Knipe, D.M. cGAS-mediated stabilization of IFI16 promotes innate signaling during herpes simplex virus infection. Proc. Natl. Acad. Sci. USA 2015, 112, 1773-1781. [CrossRef]

138. Van Lint, A.L.; Murawski, M.R.; Goodbody, R.E.; Severa, M.; Fitzgerald, K.A.; Finberg, R.W.; Knipe, D.M.; Kurt-Jones, E.A. Herpes simplex virus immediate-early ICP0 protein inhibits Toll-like receptor 2-dependent inflammatory responses and NF-kappaB signaling. J. Virol. 2010, 84, 10802-10811. [CrossRef] 
139. Lin, R.; Noyce, R.S.; Collins, S.E.; Everett, R.D.; Mossman, K.L. The herpes simplex virus ICP0 RING finger domain inhibits IRF3and IRF7-mediated activation of interferon-stimulated genes. J. Virol. 2004, 78, 1675-1684. [CrossRef]

140. Melroe, G.T.; DeLuca, N.A.; Knipe, D.M. Herpes simplex virus 1 has multiple mechanisms for blocking virus-induced interferon production. J. Virol. 2004, 78, 8411-8420. [CrossRef]

141. Melroe, G.T.; Silva, L.; Schaffer, P.A.; Knipe, D.M. Recruitment of activated IRF-3 and CBP/p300 to herpes simplex virus ICP0 nuclear foci: Potential role in blocking IFN-beta induction. Virology 2007, 360, 305-321. [CrossRef]

142. Waisner, H.; Kalamvoki, M. The ICP0 protein of herpes simplex virus 1 (HSV-1) downregulates major autophagy adaptor proteins sequestosome 1 and optineurin during the early stages of HSV-1 infection. J. Virol. 2019, 93. [CrossRef] [PubMed]

143. Kalamvoki, M.; Gu, H.; Roizman, B. Overexpression of the ubiquitin-specific protease 7 resulting from transfection or mutations in the ICP0 binding site accelerates rather than depresses herpes simplex virus 1 gene expression. J. Virol. 2012, 86, 12871-12878. [CrossRef] [PubMed]

144. Mostafa, H.H.; Thompson, T.W.; Davido, D.J. N-terminal phosphorylation sites of herpes simplex virus 1 ICP0 differentially regulate its activities and enhance viral replication. J. Virol. 2013, 87, 2109-2119. [CrossRef] [PubMed]

145. Colleran, A.; Collins, P.E.; O'Carroll, C.; Ahmed, A.; Mao, X.; McManus, B.; Kiely, P.A.; Burstein, E.; Carmody, R.J. Deubiquitination of NF-кB by ubiquitin-specific protease-7 promotes transcription. Proc. Natl. Acad. Sci. USA 2013, 110, 618-623. [CrossRef] [PubMed]

146. Liang, Y.; Kurakin, A.; Roizman, B. Herpes simplex virus 1 infected cell protein 0 forms a complex with CIN85 and Cbl and mediates the degradation of EGF receptor from cell surfaces. Proc. Natl. Acad. Sci. USA 2005, 102, 5838-5843. [CrossRef] [PubMed]

147. Deschamps, T.; Dogrammatzis, C.; Mullick, R.; Kalamvoki, M. Cbl E3 ligase mediates the removal of nectin-1 from the surface of herpes simplex virus 1-infected cells. J. Virol. 2017, 91. [CrossRef]

148. Leib, D.A.; Coen, D.M.; Bogard, C.L.; Hicks, K.A.; Yager, D.R.; Knipe, D.M.; Tyler, K.L.; Schaffer, P.A. Immediate-early regulatory gene mutants define different stages in the establishment and reactivation of herpes simplex virus latency. J. Virol. 1989, 63, 759-768. [CrossRef]

149. Halford, W.P.; Kemp, C.D.; Isler, J.A.; Davido, D.J.; Schaffer, P.A. ICP0, ICP4, or VP16 expressed from adenovirus vectors induces reactivation of latent herpes simplex virus type 1 in primary cultures of latently infected trigeminal ganglion cells. J. Virol. 2001, 75, 6143-6153. [CrossRef]

150. Harris, R.A.; Everett, R.D.; Zhu, X.X.; Silverstein, S.; Preston, C.M. Herpes simplex virus type 1 immediate-early protein Vmw110 reactivates latent herpes simplex virus type 2 in an in vitro latency system. J. Virol. 1989, 63, 3513-3515. [CrossRef]

151. Cliffe, A.R.; Arbuckle, J.H.; Vogel, J.L.; Geden, M.J.; Rothbart, S.B.; Cusack, C.L.; Strahl, B.D.; Kristie, T.M.; Deshmukh, M. Neuronal stress pathway mediating a histone methyl/phospho switch is required for herpes simplex virus reactivation. Cell Host Microbe 2015, 18, 649-658. [CrossRef] [PubMed]

152. Thompson, R.L.; Preston, C.M.; Sawtell, N.M. De novo synthesis of VP16 coordinates the exit from HSV latency in vivo. PLoS Pathog. 2009, 5, e1000352. [CrossRef] [PubMed]

153. McGeoch, D.J.; Dalrymple, M.A.; Davison, A.J.; Dolan, A.; Frame, M.C.; McNab, D.; Perry, L.J.; Scott, J.E.; Taylor, P. The complete DNA sequence of the long unique region in the genome of herpes simplx virus type 1. J. Gen. Virol. 1988, 69, 1531-1574. [CrossRef] [PubMed]

154. McKnight, J.L.; Pellett, P.E.; Jenkins, F.J.; Roizman, B. Characterization and nucleotide sequence of two herpes simplex virus 1 genes whose products modulate alpha-trans-inducing factor-dependent activation of alpha genes. J. Virol. 1987, 61, 992-1001. [CrossRef] [PubMed]

155. Vittone, V.; Diefenbach, E.; Triffett, D.; Douglas, M.W.; Cunningham, A.L.; Diefenbach, R.J. Determination of interactions between tegument proteins of herpes simplex virus type 1. J. Virol. 2005, 79, 9566-9571. [CrossRef] [PubMed]

156. Barker, D.E.; Roizman, B. Identification of three genes nonessential for growth in cell culture near the right terminus of the unique sequences of long component of herpes simplex virus 1. Virology 1990, 177, 684-691. [CrossRef]

157. Kato, K.; Daikoku, T.; Goshima, F.; Kume, H.; Yamaki, K.; Nishiyama, Y. Synthesis, subcellular localization and VP16 interaction of the herpes simplex virus type 2 UL46 gene product. Arch. Virol. 2000, 145, 2149-2162. [CrossRef] [PubMed]

158. Liu, M.; Tang, J.; Wang, X.; Yang, T.; Geller, A.I. Enhanced long-term expression from helper virus-free HSV-1 vectors packaged in the presence of deletions in genes that modulate the function of VP16, U L 46 and U L 47. J. Neurosci. Methods 2005, 145, 1-9. [CrossRef]

159. McLean, G.; Rixon, F.; Langeland, N.; Haarr, L.; Marsden, H. Identification and characterization of the virion protein products of herpes simplex virus type 1 gene UL47. J. Gen. Virol. 1990, 71, 2953-2960. [CrossRef]

160. Meredith, D.M.; Lindsay, J.A.; Halliburton, I.W.; Whittaker, G.R. Post-translational modification of the tegument proteins (VP13 and VP14) of herpes simplex virus type 1 by glycosylation and phosphorylation. J. Gen. Virol. 1991, 72, 2771-2775. [CrossRef]

161. Zhang, Y.; McKnight, J.L. Herpes simplex virus type 1 UL46 and UL47 deletion mutants lack VP11 and VP12 or VP13 and VP14, respectively, and exhibit altered viral thymidine kinase expression. J. Virol. 1993, 67, 1482-1492. [CrossRef]

162. Kopp, M.; Klupp, B.G.; Granzow, H.; Fuchs, W.; Mettenleiter, T.C. Identification and characterization of the pseudorabies virus tegument proteins UL46 and UL47: Role for UL47 in virion morphogenesis in the cytoplasm. J. Virol. 2002, 76, 8820-8833. [CrossRef] [PubMed] 
163. Strunk, U.; Saffran, H.A.; Wu, F.W.; Smiley, J.R. Role of herpes simplex virus VP11/12 tyrosine-based motifs in binding and activation of the Src family kinase Lck and recruitment of p85, Grb2, and Shc. J. Virol. 2013, 87, 11276-11286. [CrossRef] [PubMed]

164. Yanagida, N.; Yoshida, S.; Nazerian, K.; Lee, L.F. Nucleotide and predicted amino acid sequences of Marek's disease virus homologues of herpes simplex virus major tegument proteins. J. Gen. Virol. 1993, 74, 1837-1845. [CrossRef] [PubMed]

165. Carpenter, D.E.; Misra, V. The most abundant protein in bovine herpes 1 virions is a homologue of herpes simplex virus type 1 UL47. J. Gen. Virol. 1991, 72, 3077-3084. [CrossRef]

166. Whittaker, G.R.; Riggio, M.P.; Halliburton, I.W.; Killington, R.A.; Allen, G.P.; Meredith, D.M. Antigenic and protein sequence homology between VP13/14, a herpes simplex virus type 1 tegument protein, and gp10, a glycoprotein of equine herpesvirus 1 and 4. J. Virol. 1991, 65, 2320-2326. [CrossRef]

167. Verjans, G.M.; Dings, M.E.; McLauchlan, J.; van der Kooi, A.; Hoogerhout, P.; Brugghe, H.F.; Timmermans, H.A.; Baarsma, G.S.; Osterhaus, A.D. Intraocular T cells of patients with herpes simplex virus (HSV)-induced acute retinal necrosis recognize HSV tegument proteins VP11/12 and VP13/14. J. Infect. Dis. 2000, 182, 923-927. [CrossRef]

168. Zahariadis, G.; Wagner, M.J.; Doepker, R.C.; Maciejko, J.M.; Crider, C.M.; Jerome, K.R.; Smiley, J.R. Cell-type-specific tyrosine phosphorylation of the herpes simplex virus tegument protein VP11/12 encoded by gene UL46. J. Virol. 2008, 82, 6098-6108. [CrossRef]

169. Wagner, M.J.; Smiley, J.R. Herpes simplex virus requires VP11/12 to induce phosphorylation of the activation loop tyrosine (Y394) of the Src family kinase Lck in T lympocytes. J. Virol. 2009, 83, 12452-12461. [CrossRef]

170. Benetti, L.; Roizman, B. Protein kinase B/Akt is present in activated form throughout the entire replicative cycle of deltaU(S)3 mutant virus but only at early times after infection with wild-type herpes simplex virus 1. J. Virol. 2006, 80, 3341-3348. [CrossRef]

171. Wagner, M.J.; Smiley, J.R. Herpes simplex virus requires VP11/12 to activate src family kinase-phosphoinositide 3-kinase-akt signaling. J. Virol. 2011, 85, 2803-2812. [CrossRef]

172. Strunk, U.; Ramos, D.G.; Saffran, H.A.; Smiley, J.R. Role of Herpes simplex virus 1 VP11/12 tyrosine-based binding motifs for Src family kinases, p85, Grb2 and Shc in activation of the phosphoinositide 3-kinase-Akt pathway. Virology 2016, 498, 31-35. [CrossRef] [PubMed]

173. Martin, C.; Leyton, L.; Hott, M.; Arancibia, Y.; Spichiger, C.; McNiven, M.A.; Court, F.A.; Concha, M.I.; Burgos, P.V.; Otth, C. Herpes simplex virus type 1 neuronal infection perturbs golgi apparatus integrity through activation of src tyrosine kinase and dyn-2 GTPase. Front. Cell. Infect. Microbiol. 2017, 7, 371. [CrossRef] [PubMed]

174. Bello-Morales, R.; Crespillo, A.J.; Fraile-Ramos, A.; Tabarés, E.; Alcina, A.; López-Guerrero, J.A. Role of the small GTPase Rab27a during herpes simplex virus infection of oligodendrocytic cells. BMC Microbiol. 2012, 12, 265. [CrossRef] [PubMed]

175. Dong, S.; Corre, B.; Foulon, E.; Dufour, E.; Veillette, A.; Acuto, O.; Michel, F. T cell receptor for antigen induces linker for activation of T cell-dependent activation of a negative signaling complex involving Dok-2, SHIP-1, and Grb-2. J. Exp. Med. 2006, 203, 2509-2518. [CrossRef]

176. Némorin, J.-G.; Laporte, P.; Bérubé, G.; Duplay, P. p62 dok negatively regulates CD2 signaling in jurkat cells. J. Immunol. 2001, 166, 4408-4415. [CrossRef]

177. Lahmidi, S.; Strunk, U.; Smiley, J.R.; Pearson, A.; Duplay, P. Herpes simplex virus 1 infection of T cells causes VP11/12-dependent phosphorylation and degradation of the cellular protein Dok-2. Virology 2017, 511, 66-73. [CrossRef]

178. Vink, E.I.; Lee, S.; Smiley, J.R.; Mohr, I. Remodeling mTORC1 responsiveness to amino acids by the herpes simplex virus UL46 and Us3 gene products supports replication during nutrient insufficiency. J. Virol. 2018, 92, 1-10. [CrossRef]

179. Deschamps, T.; Kalamvoki, M. Evasion of the STING DNA-sensing pathway by VP11/12 of herpes simplex virus 1. J. Virol. 2017, 91. [CrossRef]

180. You, H.; Zheng, S.; Huang, Z.; Lin, Y.; Shen, Q.; Zheng, C. Herpes simplex virus 1 tegument protein ul46 inhibits tank-binding kinase 1-mediated signaling. mBio 2019, 10,1-10. [CrossRef]

181. Xu, J.; Gao, F.; Wu, J.; Zheng, H.; Tong, W.; Cheng, X.; Liu, Y.; Zhu, H.; Fu, X.; Jiang, Y.; et al. Characterization of nucleocytoplasmic shuttling of pseudorabies virus protein UL46. Front. Vet. Sci. 2020, 7, 484. [CrossRef]

182. Liu, Z.; Kato, A.; Shindo, K.; Noda, T.; Sagara, H.; Kawaoka, Y.; Arii, J.; Kawaguchi, Y. Herpes simplex virus 1 UL47 Interacts with viral nuclear egress factors UL31, UL34, and Us3 and regulates viral nuclear egress. J. Virol. 2014, 88, 4657-4667. [CrossRef] [PubMed]

183. Donnelly, M.; Elliott, G. Nuclear localization and shuttling of herpes simplex virus tegument protein VP13/14. J. Virol. 2001, 75, 2566-2574. [CrossRef]

184. Donnelly, M.; Elliott, G. Fluorescent tagging of herpes simplex virus tegument protein VP13/14 in virus infection. J. Virol. 2001, 75, 2575-2583. [CrossRef] [PubMed]

185. Yedowitz, J.C.; Kotsakis, A.; Schlegel, E.F.M.; Blaho, J.A. Nuclear localizations of the herpes simplex virus type 1 tegument proteins VP13/14, vhs, and VP16 precede VP22-dependent microtubule reorganization and VP22 nuclear import. J. Virol. 2005, 79, 4730-4743. [CrossRef] [PubMed]

186. Dobrikova, E.; Shveygert, M.; Walters, R.; Gromeier, M. Herpes simplex virus proteins ICP27 and UL47 associate with polyadenylate-binding protein and control its subcellular distribution. J. Virol. 2010, 84, 270-279. [CrossRef] [PubMed]

187. Shu, M.; Taddeo, B.; Zhang, W.; Roizman, B. Selective degradation of mRNAs by the HSV host shutoff RNase is regulated by the UL47 tegument protein. Proc. Natl. Acad. Sci. USA 2013, 110. [CrossRef] [PubMed] 
188. Heine, J.W.; Honess, R.W.; Cassai, E.; Roizman, B. Proteins specified by herpes simplex virus XII. The virion polypeptides of type 1 strains. J. Virol. 1974, 14, 640-651. [CrossRef]

189. Aints, A.; Güven, H.; Gahrton, G.; Smith, C.I.E.; Dilber, M.S. Mapping of herpes simplex virus-1 VP22 functional domains for inter- and subcellular protein targeting. Gene Ther. 2001, 8, 1051-1056. [CrossRef]

190. Morrison, E.E.; Wang, Y.F.; Meredith, D.M. Phosphorylation of structural components promotes dissociation of the herpes simplex virus type 1 tegument. J. Virol. 1998, 72, 7108-7114. [CrossRef]

191. Pomeranz, L.E.; Blaho, J.A. Assembly of infectious herpes simplex virus type 1 virions in the absence of full-length VP22. J. Virol. 2000, 74, 10041-10054. [CrossRef]

192. Van Leeuwen, H.; Okuwaki, M.; Hong, R.; Chakravarti, D.; Nagata, K.; O’Hare, P. Herpes simplex virus type 1 tegument protein VP22 interacts with TAF-I proteins and inhibits nucleosome assembly but not regulation of histone acetylation by INHAT. J. Gen. Virol. 2003, 84, 2501-2510. [CrossRef] [PubMed]

193. López, M.R.; Schlegel, E.F.M.; Wintersteller, S.; Blaho, J.A. The major tegument structural protein VP22 targets areas of dispersed nucleolin and marginalized chromatin during productive herpes simplex virus 1 infection. Virus Res. 2008, 136, 175-188. [CrossRef] [PubMed]

194. Yu, X.; Liu, L.; Wu, L.; Wang, L.; Dong, C.; Li, W.; Li, Q. Herpes simplex virus type 1 tegument protein VP22 is capable of modulating the transcription of viral TK and gC genes via interaction with viral ICP0. Biochimie 2010, 92, 1024-1030. [CrossRef] [PubMed]

195. Tanaka, M.; Kato, A.; Satoh, Y.; Ide, T.; Sagou, K.; Kimura, K.; Hasegawa, H.; Kawaguchi, Y. Herpes simplex virus 1 VP22 regulates translocation of multiple viral and cellular proteins and promotes neurovirulence. J. Virol. 2012, 86, 5264-5277. [CrossRef] [PubMed]

196. Sciortino, M.T.; Taddeo, B.; Giuffrè-Cuculletto, M.; Medici, M.A.; Mastino, A.; Roizman, B. Replication-competent herpes simplex virus 1 isolates selected from cells transfected with a bacterial artificial chromosome DNA lacking only the UL49 gene vary with respect to the defect in the UL41 gene encoding host shutoff RNase. J. Virol. 2007, 81, 10924-10932. [CrossRef] [PubMed]

197. Mbong, E.F.; Woodley, L.; Dunkerley, E.; Schrimpf, J.E.; Morrison, L.A.; Duffy, C. Deletion of the herpes simplex virus 1 UL49 gene results in mRNA and protein translation defects that are complemented by secondary mutations in UL41. J. Virol. 2012, 86, 12351-12361. [CrossRef]

198. Duffy, C.; Mbong, E.F.; Baines, J.D. VP22 of herpes simplex virus 1 promotes protein synthesis at late times in infection and accumulation of a subset of viral mRNAs at early times in infection. J. Virol. 2009, 83, 1009-1017. [CrossRef]

199. Brignati, M.J.; Loomis, J.S.; Wills, J.W.; Courtney, R.J. Membrane association of VP22, a herpes simplex virus type 1 tegument protein. J. Virol. 2003, 77, 4888-4898. [CrossRef]

200. O’Regan, K.J.; Brignati, M.J.; Murphy, M.A.; Bucks, M.A.; Courtney, R.J. Virion incorporation of the herpes simplex virus type 1 tegument protein VP22 is facilitated by trans-Golgi network localization and is independent of interaction with glycoprotein E. Virology 2010, 405, 176-192. [CrossRef]

201. Leslie, J.; Rixon, F.J.; Mclauchlan, J. Overexpression of the herpes simplex virus type 1 tegument protein VP22 increases its incorporation into virus particles. Virology 1996, 68, 60-68. [CrossRef]

202. Chi, J.H.I.; Harley, C.A.; Mukhopadhyay, A.; Wilson, D.W. The cytoplasmic tail of herpes simplex virus envelope glycoprotein D binds to the tegument protein VP22 and to capsids. J. Gen. Virol. 2005, 86, 253-261. [CrossRef] [PubMed]

203. Starkey, J.L.; Han, J.; Chadha, P.; Marsh, J.A.; Wills, J.W. Elucidation of the block to herpes simplex virus egress in the absence of tegument protein UL16 reveals a novel interaction with VP22. J. Virol. 2014, 88, 110-119. [CrossRef] [PubMed]

204. O’Regan, K.J.; Bucks, M.A.; Murphy, M.A.; Wills, J.W.; Courtney, R.J. A conserved region of the herpes simplex virus type 1 tegument protein VP22 facilitates interaction with the cytoplasmic tail of glycoprotein E (gE). Virology 2007, 358, 192-200. [CrossRef] [PubMed]

205. O'Regan, K.J.; Murphy, M.A.; Bucks, M.A.; Wills, J.W.; Courtney, R.J. Incorporation of the herpes simplex virus type 1 tegument protein VP22 into the virus particle is independent of interaction with VP16. Virology 2007, 369, 263-280. [CrossRef]

206. Duffy, C.; LaVail, J.H.; Tauscher, A.N.; Wills, E.G.; Blaho, J.A.; Baines, J.D. Characterization of a UL49-null mutant: VP22 of herpes simplex virus type 1 facilitates viral spread in cultured cells and the mouse cornea. J. Virol. 2006, 80, 8664-8675. [CrossRef]

207. Elliott, G.; Mouzakitis, G.; O'Hare, P. VP16 interacts via its activation domain with VP22, a tegument protein of herpes simplex virus, and is relocated to a novel macromolecular assembly in coexpressing cells. J. Virol. 1995, 69, 7932-7941. [CrossRef]

208. Maringer, K.; Stylianou, J.; Elliott, G. A Network of protein interactions around the herpes simplex virus tegument protein VP22. J. Virol. 2012, 86, 12971-12982. [CrossRef]

209. Kotsakis, A.; Pomeranz, L.E.; Blouin, A.; Blaho, J.A. Microtubule reorganization during herpes simplex virus type 1 infection facilitates the nuclear localization of VP22, a major virion tegument protein. J. Virol. 2001, 75, 8697-8711. [CrossRef]

210. Blouin, A.; Blaho, J.A. Assessment of the subcellular localization of the herpes simplex virus structural protein VP22 in the absence of other viral gene products. Virus Res. 2001, 81, 57-68. [CrossRef]

211. Van Leeuwen, H.; Elliott, G.; O'Hare, P. Evidence of a role for nonmuscle myosin II in herpes simplex virus type 1 egress. J. Virol. 2002, 76, 3471-3481. [CrossRef]

212. Liu, J.; Gallo, R.M.; Duffy, C.; Brutkiewicz, R.R. A VP22-null HSV-1 is impaired in inhibiting CD1d-mediated antigen presentation. Viral Immunol. 2016, 29, 409-416. [CrossRef] [PubMed] 
213. Horan, K.A.; Hansen, K.; Jakobsen, M.R.; Holm, C.K.; Søby, S.; Unterholzner, L.; Thompson, M.; West, J.A.; Iversen, M.B.; Rasmussen, S.B.; et al. Proteasomal degradation of herpes simplex virus capsids in macrophages releases DNA to the cytosol for recognition by DNA sensors. J. Immunol. 2013, 190, 2311-2319. [CrossRef] [PubMed]

214. Maruzuru, Y.; Ichinohe, T.; Sato, R.; Miyake, K.; Okano, T.; Suzuki, T.; Koshiba, T.; Koyanagi, N.; Tsuda, S.; Watanabe, M.; et al. Herpes simplex virus 1 VP22 inhibits AIM2-dependent inflammasome activation to enable efficient viral replication. Cell Host Microbe 2018, 23, 254-265. [CrossRef] [PubMed]

215. Elliott, G.; O'Hare, P. Intercellular trafficking and protein delivery by a herpesvirus structural protein. Cell 1997, 88, 223-233. [CrossRef]

216. Sciortino, M.T.; Taddeo, B.; Poon, A.P.W.; Mastino, A.; Roizman, B. Of the three tegument proteins that package mRNA in herpes simplex virions, one (VP22) transports the mRNA to uninfected cells for expression prior to viral infection. Proc. Natl. Acad. Sci. USA 2002, 99, 8318-8323. [CrossRef]

217. Phelan, A.; Elliott, G.; Hare, P.O. Intercellular delivery of functional p53 by the herpesvirus protein VP22. Nat. Biotechnol. 1998, 16, 440-443. [CrossRef] [PubMed]

218. Fang, B.; Xu, B.; Koch, P.; Roth, J.A. Intercellular trafficking of VP22-GFP fusion proteins is not observed in cultured mammalian cells. Gene Ther. 1998, 5, 1420-1424. [CrossRef]

219. Dilber, M.S.; Phelan, A.; Aints, A.; Mohamed, A.J.; Elliott, G.; Edvard Smith, C.I.; O'Hare, P. Intercellular delivery of thymidine kinase prodrug activating enzyme by the herpes simplex virus protein, VP22. Gene Ther. 1999, 6, 12-21. [CrossRef]

220. Sheridan, P.J.; Lawrie, A.; Crossman, D.C.; Holt, C.M.; Newman, C.M. VP22-mediated intercellular transport correlates with enhanced biological activity of MybEngrailed but not (HSV-I) thymidine kinase fusion proteins in primary vascular cells following non-viral transfection. J. Gene Med. 2005, 7, 375-385. [CrossRef]

221. Hakkarainen, T.; Wahlfors, T.; Meriläinen, O.; Loimas, S.; Hemminki, A.; Wahlfors, J. VP22 does not significantly enhance enzyme prodrug cancer gene therapy as a part of a VP22-HSVTk-GFP triple fusion construct. J. Gene Med. 2005, 7, 898-907. [CrossRef]

222. Beerens, A.M.J.; Rots, M.G.; de Vries, E.F.J.; Haisma, H.J. Fusion of herpes simplex virus thymidine kinase to VP22 does not result in intercellular trafficking of the protein. Int. J. Mol. Med. 2007, 19, 841-849. [CrossRef] [PubMed]

223. Aints, A.; Dilber, M.S.; Smith, C.I.E. Intercellular spread of GFP-VP22. J. Gene Med. 1999, 1, 275-279. [CrossRef]

224. Dogrammatzis, C.; Deschamps, T.; Kalamvoki, M. Biogenesis of extracellular vesicles during herpes simplex virus 1 infection: Role of the CD63 tetraspanin. J. Virol. 2018, 93. [CrossRef] [PubMed]

225. Dogrammatzis, C.; Saleh, S.; Deighan, C.; Kalamvoki, M. Diverse populations of extracellular vesicles with opposite functions during herpes simplex virus 1 infection. J. Virol. 2020, 95, e02357-20.

226. Greco, O.; Joiner, M.C.; Doleh, A.; Scott, S.D. VP22-mediated intercellular transport for suicide gene therapy under oxic and hypoxic conditions. Gene Ther. 2005, 12, 974-979. [CrossRef] [PubMed]

227. Liu, C.S.; Kong, B.H.; Xia, H.H.Q.; Ellem, K.A.O.; Wei, M.Q. VP22 enhanced intercellular trafficking of HSV thymidine kinase reduced the level of ganciclovir needed to cause suicide cell death. J. Gene Med. 2001, 3, 145-152. [CrossRef] [PubMed]

228. Wybranietz, W.A.; Groß, C.D.; Phelan, A.; O’Hare, P.; Spiegel, M.; Graepler, F.; Bitzer, M.; Stähler, P.; Gregor, M.; Lauer, U.M. Enhanced suicide gene effect by adenoviral transduction of aVP22-cytosine deaminase (CD) fusion gene. Gene Ther. 2001, 8 , 1654-1664. [CrossRef]

229. Lee, K.C.; Hamstra, D.A.; Bullarayasamudram, S.; Bhojani, M.S.; Moffat, B.A.; Dornfeld, K.J.; Ross, B.D.; Rehemtulla, A. Fusion of the HSV-1 tegument protein vp22 to cytosine deaminase confers enhanced bystander effect and increased therapeutic benefit. Gene Ther. 2006, 13, 127-137. [CrossRef]

230. Cashman, S.M.; Sadowski, S.L.; Morris, D.J.; Frederick, J.; Kumar-Singh, R. Intercellular trafficking of adenovirus-delivered HSV VP22 from the retinal pigment epithelium to the photoreceptors-Implications for gene therapy. Mol. Ther. 2002, 6, 813-823. [CrossRef]

231. Kretz, A.; Wybranietz, W.A.; Hermening, S.; Lauer, U.M.; Isenmann, S. HSV-1 VP22 augments adenoviral gene transfer to CNS neurons in the retina and striatum in vivo. Mol. Ther. 2003, 7, 659-669. [CrossRef]

232. Xiong, F.; Xiao, S.; Peng, F.; Zheng, H.; Yu, M.; Ruan, Y.; Li, W.; Shang, Y.; Zhao, C.; Zhou, W.; et al. Herpes simplex virus VP22 enhances adenovirus-mediated microdystrophin gene transfer to skeletal muscles in dystrophin-deficient (mdx) mice. Hum. Gene Ther. 2007, 18, 490-501. [CrossRef] [PubMed]

233. Yu, X.; Wang, Y.; Xia, Y.; Zhang, L.; Yang, Q.; Lei, J. A DNA vaccine encoding VP22 of herpes simplex virus type I (HSV-1) and OprF confers enhanced protection from Pseudomonas aeruginosa in mice. Vaccine 2016, 34, 4399-4405. [CrossRef] [PubMed]

234. Hung, C.-F.; Cheng, W.-F.; Chai, C.-Y.; Hsu, K.-F.; He, L.; Ling, M.; Wu, T.-C. Improving vaccine potency through intercellular spreading and enhanced MHC class I presentation of antigen. J. Immunol. 2001, 166, 5733-5740. [CrossRef] [PubMed]

235. Honess, R.W.; Roizman, B. Proteins specified by herpes simplex virus. XI. Identification and relative molar rates of synthesis of structural and nonstructural herpes virus polypeptides in the infected cell. J. Virol. 1973, 12, 1347-1365. [CrossRef] [PubMed]

236. Post, L.E.; Roizman, B. A generalized technique for deletion of specific genes in large genomes: A gene 22 of herpes simplex virus 1 is not essential for growth. Cell 1981, 25, 227-232. [CrossRef]

237. Meignier, B.; Longnecker, R.; Mavromara-Nazos, P.; Sears, A.E.; Roizman, B. Virulence of and establishment of latency by genetically engineered deletion mutants of herpes simplex virus I. Virology 1988, 162, 251-254. [CrossRef] 
238. Sears, A.E.; Halliburton, I.W.; Meignier, B.; Silver, S.; Roizman, B. Herpes simplex virus 1 mutant deleted in the alpha 22 gene: Growth and gene expression in permissive and restrictive cells and establishment of latency in mice. J. Virol. 1985, 55, 338-346. [CrossRef]

239. Poffenberger, K.L.; Raichlen, P.E.; Herman, R.C. In vitro characterization of a herpes simplex virus type 1 ICP22 deletion mutant. Virus Genes 1993, 7, 171-186. [CrossRef]

240. Jacquemont, B.; Verrier, B.; Epstein, A.L.; Machuca, I. Expression of immediate-early genes in herpes simplex virus type 1-infected XC cells: Lack of ICP22 (68K) polypeptide. J. Gen. Virol. 1984, 65, 1331-1340. [CrossRef]

241. Orlando, J.S.; Astor, T.L.; Rundle, S.A.; Schaffer, P.A. The products of the herpes simplex virus type 1 immediate-early US1/US1.5 genes downregulate levels of S-phase-specific cyclins and facilitate virus replication in S-phase Vero cells. J. Virol. 2006, 80, 4005-4016. [CrossRef]

242. Ogle, W.O.; Roizman, B. Functional anatomy of herpes simplex virus 1 overlapping genes encoding infected-cell protein 22 and US1.5 protein. J. Virol. 1999, 73, 4305-4315. [CrossRef] [PubMed]

243. Poffenberger, K.L.; Idowu, A.D.; Fraser-Smith, E.B.; Raichlen, P.E.; Herman, R.C. A herpes simplex virus type 1 ICP22 deletion mutant is altered for virulence and latency in vivo. Arch. Virol. 1994, 139, 111-119. [CrossRef] [PubMed]

244. Orlando, J.S.; Balliet, J.W.; Kushnir, A.S.; Astor, T.L.; Kosz-Vnenchak, M.; Rice, S.A.; Knipe, D.M.; Schaffer, P.A. ICP22 is required for wild-type composition and infectivity of herpes simplex virus type 1 virions. J. Virol. 2006, 80, 9381-9390. [CrossRef] [PubMed]

245. Brandt, C.R.; Kolb, A.W. Tyrosine 116 of the herpes simplex virus type 1 IEalpha22 protein is an ocular virulence determinant and potential phosphorylation site. Invest. Ophthalmol. Vis. Sci. 2003, 44, 4601-4607. [CrossRef]

246. Bowles, R.N.; Blaho, J.A. A truncation mutation of the neurovirulence ICP22 protein produced by a recombinant HSV-1 generated by bacterial artificial chromosome technology targets infected cell nuclei. J. Neurovirol. 2011, 17, 559-569. [CrossRef]

247. Holden, V.R.; Zhao, Y.; Thompson, Y.; Caughman, G.B.; Smith, R.H.; O'Callaghan, D.J. Characterization of the regulatory function of the ICP22 protein of equine herpesvirus type 1. Virology 1995, 210, 273-282. [CrossRef]

248. Kost, R.G.; Kupinsky, H.; Straus, S.E. Varicella-zoster virus gene 63: Transcript mapping and regulatory activity. Virology 1995, 209, 218-224. [CrossRef]

249. Heineman, T.C.; Cohen, J.I. The varicella-zoster virus (VZV) open reading frame 47 (ORF47) protein kinase is dispensable for viral replication and is not required for phosphorylation of ORF63 protein, the VZV homolog of herpes simplex virus ICP22. J. Virol. 1995, 69, 7367-7370. [CrossRef]

250. Gray, W.L.; Gusick, N.J.; Ek-Kommonen, C.; Kempson, S.E.; Fletcher, T.M. 3rd The inverted repeat regions of the simian varicella virus and varicella-zoster virus genomes have a similar genetic organization. Virus Res. 1995, 39, 181-193. [CrossRef]

251. Cai, M.; Jiang, S.; Zeng, Z.; Li, X.; Mo, C.; Yang, Y.; Chen, C.; Xie, P.; Bian, Y.; Wang, J.; et al. Probing the nuclear import signal and nuclear transport molecular determinants of PRV ICP22. Cell Biosci. 2016, 6, 3. [CrossRef]

252. Blaho, J.A.; Mitchell, C.; Roizman, B. Guanylylation and adenylylation of the alpha regulatory proteins of herpes simplex virus require a viral beta or gamma function. J. Virol. 1993, 67, 3891-3900. [CrossRef] [PubMed]

253. Purves, F.C.; Roizman, B. The UL13 gene of herpes simplex virus 1 encodes the functions for posttranslational processing associated with phosphorylation of the regulatory protein alpha 22. Proc. Natl. Acad. Sci. USA 1992, 89, 7310-7314. [CrossRef] [PubMed]

254. Purves, F.C.; Ogle, W.O.; Roizman, B. Processing of the herpes simplex virus regulatory protein alpha 22 mediated by the UL13 protein kinase determines the accumulation of a subset of alpha and gamma mRNAs and proteins in infected cells. Proc. Natl. Acad. Sci. USA 1993, 90, 6701-6705. [CrossRef] [PubMed]

255. Blaho, J.A.; Zong, C.S.; Mortimer, K.A. Tyrosine phosphorylation of the herpes simplex virus type 1 regulatory protein ICP22 and a cellular protein which shares antigenic determinants with ICP22. J. Virol. 1997, 71, 9828-9832. [CrossRef]

256. O'Toole, J.M.; Aubert, M.; Kotsakis, A.; Blaho, J.A. Mutation of the protein tyrosine kinase consensus site in the herpes simplex virus 1 alpha22 gene alters ICP22 posttranslational modification. Virology 2003, 305, 153-167. [CrossRef]

257. Stelz, G.; Rücker, E.; Rosorius, O.; Meyer, G.; Stauber, R.H.; Spatz, M.; Eibl, M.M.; Hauber, J. Identification of two nuclear import signals in the alpha-gene product ICP22 of herpes simplex virus 1. Virology 2002, 295, 360-370. [CrossRef]

258. Ng, T.I.; Chang, Y.E.; Roizman, B. Infected cell protein 22 of herpes simplex virus 1 regulates the expression of virion host shutoff gene U(L)41. Virology 1997, 234, 226-234. [CrossRef]

259. Advani, S.J.; Brandimarti, R.; Weichselbaum, R.R.; Roizman, B. The disappearance of cyclins A and B and the increase in activity of the G(2)/M-phase cellular kinase cdc2 in herpes simplex virus 1-infected cells require expression of the alpha22/U(S)1.5 and $\mathrm{U}(\mathrm{L}) 13$ viral genes. J. Virol. 2000, 74, 8-15. [CrossRef]

260. Advani, S.J.; Weichselbaum, R.R.; Roizman, B. The role of cdc2 in the expression of herpes simplex virus genes. Proc. Natl. Acad. Sci. USA 2000, 97, 10996-11001. [CrossRef]

261. Advani, S.J.; Weichselbaum, R.R.; Roizman, B. Herpes simplex virus 1 activates cdc2 to recruit topoisomerase II alpha for post-DNA synthesis expression of late genes. Proc. Natl. Acad. Sci. USA 2003, 100, 4825-4830. [CrossRef]

262. Smith-Donald, B.A.; Roizman, B. The interaction of herpes simplex virus 1 regulatory protein ICP22 with the cdc $25 \mathrm{C}$ phosphatase is enabled in vitro by viral protein kinases US3 and UL13. J. Virol. 2008, 82, 4533-4543. [CrossRef] [PubMed]

263. Rice, S.A.; Long, M.C.; Lam, V.; Schaffer, P.A.; Spencer, C.A. Herpes simplex virus immediate-early protein ICP22 is required for viral modification of host RNA polymerase II and establishment of the normal viral transcription program. J. Virol. 1995, 69, 5550-5559. [CrossRef] [PubMed] 
264. Rice, S.A.; Long, M.C.; Lam, V.; Spencer, C.A. RNA polymerase II is aberrantly phosphorylated and localized to viral replication compartments following herpes simplex virus infection. J. Virol. 1994, 68, 988-1001. [CrossRef] [PubMed]

265. Spencer, C.A.; Dahmus, M.E.; Rice, S.A. Repression of host RNA polymerase II transcription by herpes simplex virus type 1. J. Virol. 1997, 71, 2031-2040. [CrossRef] [PubMed]

266. Jenkins, H.L.; Spencer, C.A. RNA polymerase II holoenzyme modifications accompany transcription reprogramming in herpes simplex virus type 1-infected cells. J. Virol. 2001, 75, 9872-9884. [CrossRef] [PubMed]

267. Long, M.C.; Leong, V.; Schaffer, P.A.; Spencer, C.A.; Rice, S.A. ICP22 and the UL13 protein kinase are both required for herpes simplex virus-induced modification of the large subunit of RNA polymerase II. J. Virol. 1999, 73, 5593-5604. [CrossRef] [PubMed]

268. Bastian, T.W.; Rice, S.A. Identification of sequences in herpes simplex virus type 1 ICP22 that influence RNA polymerase II modification and viral late gene expression. J. Virol. 2009, 83, 128-139. [CrossRef]

269. Bowman, J.J.; Schaffer, P.A. Origin of expression of the herpes simplex virus type 1 protein U(S)1.5. J. Virol. 2009, 83, 9183-9194. [CrossRef] [PubMed]

270. Leopardi, R.; Ward, P.L.; Ogle, W.O.; Roizman, B. Association of herpes simplex virus regulatory protein ICP22 with transcriptional complexes containing EAP, ICP4, RNA polymerase II, and viral DNA requires posttranslational modification by the U(L)13 proteinkinase. J. Virol. 1997, 71, 1133-1139. [CrossRef]

271. Jahedi, S.; Markovitz, N.S.; Filatov, F.; Roizman, B. Colocalization of the herpes simplex virus 1 UL4 protein with infected cell protein 22 in small, dense nuclear structures formed prior to onset of DNA synthesis. J. Virol. 1999, 73, 5132-5138. [CrossRef]

272. Wu, N.; Watkins, S.C.; Schaffer, P.A.; DeLuca, N.A. Prolonged gene expression and cell survival after infection by a herpes simplex virus mutant defective in the immediate-early genes encoding ICP4, ICP27, and ICP22. J. Virol. 1996, 70, 6358-6369. [CrossRef] [PubMed]

273. Cun, W.; Chen, J.; Zhang, Y.; Liu, L.; Li, Q. Analysis of the cellular localization of herpes simplex virus 1 immediate-early protein ICP22. Virol. Sin. 2010, 25, 158-167. [CrossRef]

274. Durand, L.O.; Roizman, B. Role of cdk9 in the optimization of expression of the genes regulated by ICP22 of herpes simplex virus 1. J. Virol. 2008, 82, 10591-10599. [CrossRef] [PubMed]

275. Ou, M.; Sandri-Goldin, R.M. Inhibition of cdk9 during herpes simplex virus 1 infection impedes viral transcription. PLoS ONE 2013, 8, e79007. [CrossRef] [PubMed]

276. Fraser, K.A.; Rice, S.A. Herpes simplex virus immediate-early protein ICP22 triggers loss of serine 2-phosphorylated RNA polymerase II. J. Virol. 2007, 81, 5091-5101. [CrossRef]

277. Fraser, K.A.; Rice, S.A. Herpes simplex virus type 1 infection leads to loss of serine-2 phosphorylation on the carboxyl-terminal domain of RNA polymerase II. J. Virol. 2005, 79, 11323-11334. [CrossRef]

278. Guo, L.; Wu, W.; Liu, L.; Wang, L.; Zhang, Y.; Wu, L.; Guan, Y.; Li, Q. Herpes simplex virus 1 ICP22 inhibits the transcription of viral gene promoters by binding to and blocking the recruitment of P-TEFb. PLoS ONE 2012, 7, e45749. [CrossRef]

279. Zaborowska, J.; Baumli, S.; Laitem, C.; O'Reilly, D.; Thomas, P.H.; O'Hare, P.; Murphy, S. Herpes simplex virus 1 (HSV-1) ICP22 protein directly interacts with cyclin-dependent kinase (CDK)9 to inhibit RNA polymerase II transcription elongation. PLoS ONE 2014, 9, e107654. [CrossRef]

280. Prod'hon, C.; Machuca, I.; Berthomme, H.; Epstein, A.; Jacquemont, B. Characterization of regulatory functions of the HSV-1 immediate-early protein ICP22. Virology 1996, 226, 393-402. [CrossRef]

281. Kwun, H.J.; Yim, S.W.; Lee, D.H.; Jang, K.L. Activation of the thymidine kinase promoter by herpes simplex virus type 1 immediate early proteins. Mol. Cells 1999, 9, 277-280.

282. Poon, A.P.W.; Roizman, B. Herpes simplex virus 1 ICP22 regulates the accumulation of a shorter mRNA and of a truncated US3 protein kinase that exhibits altered functions. J. Virol. 2005, 79, 8470-8479. [CrossRef] [PubMed]

283. Zhang, Y.; Yang, Z.; Cao, Y.; Zhang, S.; Li, H.; Huang, Y.; Ding, Y.-Q.; Liu, X. The Hsp40 family chaperone protein DnaJB6 enhances Schlafen1 nuclear localization which is critical for promotion of cell-cycle arrest in T-cells. Biochem. J. 2008, 413, 239-250. [CrossRef] [PubMed]

284. Sullivan, C.S.; Pipas, J.M. The virus-chaperone connection. Virology 2001, 287, 1-8. [CrossRef] [PubMed]

285. Adlakha, M.; Livingston, C.M.; Bezsonova, I.; Weller, S.K. The herpes simplex virus 1 immediate early protein ICP22 is a functional mimic of a cellular J protein. J. Virol. 2020, 94. [CrossRef] [PubMed]

286. Bastian, T.W.; Livingston, C.M.; Weller, S.K.; Rice, S.A. Herpes simplex virus type 1 immediate-early protein ICP22 is required for VICE domain formation during productive viral infection. J. Virol. 2010, 84, 2384-2394. [CrossRef] [PubMed]

287. Burch, A.D.; Weller, S.K. Herpes simplex virus type 1 DNA polymerase requires the mammalian chaperone hsp 90 for proper localization to the nucleus. J. Virol. 2005, 79, 10740-10749. [CrossRef]

288. Burch, A.D.; Weller, S.K. Nuclear sequestration of cellular chaperone and proteasomal machinery during herpes simplex virus type 1 infection. J. Virol. 2004, 78, 7175-7185. [CrossRef]

289. Livingston, C.M.; Ifrim, M.F.; Cowan, A.E.; Weller, S.K. Virus-Induced Chaperone-Enriched (VICE) domains function as nuclear protein quality control centers during HSV-1 infection. PLoS Pathog. 2009, 5, e1000619. [CrossRef]

290. Mostafa, H.H.; Davido, D.J. Herpes simplex virus 1 ICP22 but not US 1.5 is required for efficient acute replication in mice and VICE domain formation. J. Virol. 2013, 87, 13510-13519. [CrossRef]

291. Maruzuru, Y.; Shindo, K.; Liu, Z.; Oyama, M.; Kozuka-Hata, H.; Arii, J.; Kato, A.; Kawaguchi, Y. Role of herpes simplex virus 1 immediate early protein ICP22 in viral nuclear egress. J. Virol. 2014, 88, 7445-7454. [CrossRef] 
292. Matundan, H.; Ghiasi, H. Herpes simplex virus 1 ICP22 suppresses CD80 Expression by murine dendritic cells. J. Virol. 2019, 93. [CrossRef] [PubMed]

293. Tormanen, K.; Wang, S.; Ghiasi, H. CD80 plays a critical role in increased inflammatory responses in herpes simplex virus 1-infected mouse corneas. J. Virol. 2020, 94. [CrossRef] [PubMed]

294. Matundan, H.H.; Jaggi, U.; Wang, S.; Ghiasi, H. Loss of ICP22 in HSV-1 elicits immune infiltration and maintains stromal keratitis despite reduced primary and latent virus infectivity. Invest. Ophthalmol. Vis. Sci. 2019, 60, 3398-3406. [CrossRef] [PubMed]

295. Zhang, M.; Fu, M.; Li, M.; Hu, H.; Gong, S.; Hu, Q. Herpes simplex virus type 2 inhibits type I IFN signaling mediated by the novel E3 ubiquitin protein ligase activity of viral protein ICP22. J. Immunol. 2020, 205, 1281-1292. [CrossRef] [PubMed]

296. Chou, J.; Roizman, B. The terminal a sequence of the herpes simplex virus genome contains the promoter of a gene located in the repeat sequences of the L component. J. Virol. 1986, 57, 629-637. [CrossRef] [PubMed]

297. Ackermann, M.; Chou, J.; Sarmiento, M.; Lerner, R.A.; Roizman, B. Identification by antibody to a synthetic peptide of a protein specified by a diploid gene located in the terminal repeats of the L component of herpes simplex virus genome. J. Virol. 1986, 58, 843-850. [CrossRef]

298. Taha, M.; Clements, G.; Brown, S. A variant of herpes simplex virus type 2 strain HG52 with a $1.5 \mathrm{~kb}$ deletion in RL between 0 to 0.02 and 0.81 to 0.83 map units is non-neurovirulent for mice. J. Gen. Virol. 1989, 70, 705-716. [CrossRef]

299. Taha, M.Y.; Clements, G.B.; Brown, S.M. The herpes simplex virus type 2 (HG52) variant JH2604 has a 1488 bp deletion which eliminates neurovirulence in mice. J. Gen. Virol. 1989, 70, 3073-3078. [CrossRef]

300. Whitley, R.J.; Kern, E.R.; Chatterjee, S.; Chou, J.; Roizman, B. Replication, establishment of latency, and induced reactivation of herpes simplex virus gamma 134.5 deletion mutants in rodent models. J. Clin. Investig. 1993, 91, 2837-2843. [CrossRef]

301. MacLean, A.R.; Ul-Fareed, M.; Robertson, L.; Harland, J.; Brown, S.M. Herpes simplex virus type 1 deletion variants 1714 and 1716 pinpoint neurovirulence-related sequences in Glasgow strain 17+ between immediate early gene 1 and the "a" sequence. J. Gen. Virol. 1991, 72, 631-639. [CrossRef]

302. Chou, J.; Kern, E.R.; Whitley, R.J.; Roizman, B. Mapping of herpes simplex virus-1 neurovirulence to gamma 134.5, a gene nonessential for growth in culture. Science 1990, 250, 1262-1266. [CrossRef] [PubMed]

303. Robertson, L.M.; MacLean, A.R.; Brown, S.M. Peripheral replication and latency reactivation kinetics of the non-neurovirulent herpes simplex virus type 1 variant 1716. J. Gen. Virol. 1992, 73, 967-970. [CrossRef]

304. Dolan, A.; McKie, E.; MacLean, A.R.; McGeoch, D.J. Status of the ICP34.5 gene in herpes simplex virus type 1 strain 17. J. Gen. Virol. 1992, 73, 971-973. [CrossRef] [PubMed]

305. Perng, G.C.; Thompson, R.L.; Sawtell, N.M.; Taylor, W.E.; Slanina, S.M.; Ghiasi, H.; Kaiwar, R.; Nesburn, A.B.; Wechsler, S.L. An avirulent ICP34.5 deletion mutant of herpes simplex virus type 1 is capable of in vivo spontaneous reactivation. J. Virol. 1995, 69, 3033-3041. [CrossRef] [PubMed]

306. Thompson, R.L.; Stevens, J.G. Biological characterization of a herpes simplex virus intertypic recombinant which is completely and specifically non-neurovirulent. Virology 1983, 131, 171-179. [CrossRef]

307. Bolovan, C.A.; Sawtell, N.M.; Thompson, R.L. ICP34.5 mutants of herpes simplex virus type 1 strain 17 syn+ are attenuated for neurovirulence in mice and for replication in confluent primary mouse embryo cell cultures. J. Virol. 1994, 68, 48-55. [CrossRef] [PubMed]

308. Perng, G.C.; Ghiasi, H.; Slanina, S.M.; Nesburn, A.B.; Wechsler, S.L. High-dose ocular infection with a herpes simplex virus type $1 \mathrm{ICP} 34.5$ deletion mutant produces no corneal disease or neurovirulence yet results in wild-type levels of spontaneous reactivation. J. Virol. 1996, 70, 2883-2893. [CrossRef]

309. Mattila, R.K.; Harila, K.; Kangas, S.M.; Paavilainen, H.; Heape, A.M.; Mohr, I.J.; Hukkanen, V. An investigation of herpes simplex virus type 1 latency in a novel mouse dorsal root ganglion model suggests a role for ICP34.5 in reactivation. J. Gen. Virol. 2015, 96, 2304-2313. [CrossRef]

310. Brown, S.M.; Harland, J.; MacLean, A.R.; Podlech, J.; Clements, J.B. Cell type and cell state determine differential in vitro growth of non-neurovirulent ICP34.5-negative herpes simplex virus types 1 and 2. J. Gen. Virol. 1994, 75, 2367-2377. [CrossRef]

311. Kesari, S.; Lasner, T.M.; Balsara, K.R.; Randazzo, B.P.; Lee, V.M.; Trojanowski, J.Q.; Fraser, N.W. A neuroattenuated ICP34.5deficient herpes simplex virus type 1 replicates in ependymal cells of the murine central nervous system. J. Gen. Virol. 1998, 79, 525-536. [CrossRef]

312. Mao, H.; Rosenthal, K.S. Strain-dependent structural variants of herpes simplex virus type 1 ICP34.5 determine viral plaque size, efficiency of glycoprotein processing, and viral release and neuroinvasive disease potential. J. Virol. 2003, 77, 3409-3417. [CrossRef] [PubMed]

313. Brown, S.M.; MacLean, A.R.; McKie, E.A.; Harland, J. The herpes simplex virus virulence factor ICP34.5 and the cellular protein MyD116 complex with proliferating cell nuclear antigen through the 63-amino-acid domain conserved in ICP34.5, MyD116, and GADD34. J. Virol. 1997, 71, 9442-9449. [CrossRef] [PubMed]

314. Chou, J.; Roizman, B. Herpes simplex virus 1 gamma(1)34.5 gene function, which blocks the host response to infection, maps in the homologous domain of the genes expressed during growth arrest and DNA damage. Proc. Natl. Acad. Sci. USA 1994, 91, 5247-5251. [CrossRef]

315. Novoa, I.; Zeng, H.; Harding, H.P.; Ron, D. Feedback inhibition of the unfolded protein response by GADD34-mediated dephosphorylation of eIF2 $\alpha$. J. Cell Biol. 2001, 153, 1011-1022. [CrossRef]

316. McGeoch, D.J.; Barnett, B.C. Neurovirulence factor. Nature 1991, 353, 609. [CrossRef] 
317. Chou, J.; Chen, J.J.; Gross, M.; Roizman, B. Association of a M(r) 90,000 phosphoprotein with protein kinase PKR in cells exhibiting enhanced phosphorylation of translation initiation factor eIF-2 alpha and premature shutoff of protein synthesis after infection with gamma 134.5- mutants of herpes si. Proc. Natl. Acad. Sci. USA 1995, 92, 10516-10520. [CrossRef] [PubMed]

318. Chou, J.; Roizman, B. The gamma 1(34.5) gene of herpes simplex virus 1 precludes neuroblastoma cells from triggering total shutoff of protein synthesis characteristic of programed cell death in neuronal cells. Proc. Natl. Acad. Sci. USA 1992, 89, 3266-3270. [CrossRef]

319. Cassady, K.A.; Gross, M.; Roizman, B. The second-site mutation in the herpes simplex virus recombinants lacking the gamma134.5 genes precludes shutoff of protein synthesis by blocking the phosphorylation of eIF-2alpha. J. Virol. 1998, 72, 7005-7011. [CrossRef]

320. Ward, S.L.; Scheuner, D.; Poppers, J.; Kaufman, R.J.; Mohr, I.; Leib, D.A. In vivo replication of an ICP34.5 second-site suppressor mutant following corneal infection correlates with in vitro regulation of eIF2 alpha phosphorylation. J. Virol. 2003, 77, 4626-4634 [CrossRef]

321. Tallóczy, Z.; Virgin, H.W., IV; Levine, B. PKR-dependent autophagic degradation of herpes simplex virus type 1. Autophagy 2006, 2, 24-29. [CrossRef]

322. Wylie, K.M.; Schrimpf, J.E.; Morrison, L.A. Increased eIF2alpha phosphorylation attenuates replication of herpes simplex virus 2 vhs mutants in mouse embryonic fibroblasts and correlates with reduced accumulation of the PKR antagonist ICP34.5. J. Virol. 2009, 83, 9151-9162. [CrossRef]

323. He, B.; Gross, M.; Roizman, B. The gamma(1)34.5 protein of herpes simplex virus 1 complexes with protein phosphatase 1alpha to dephosphorylate the alpha subunit of the eukaryotic translation initiation factor 2 and preclude the shutoff of protein synthesis by double-stranded RNA-activ. Proc. Natl. Acad. Sci. USA 1997, 94, 843-848. [CrossRef] [PubMed]

324. Cheng, G.; Brett, M.-E.; He, B. Val193 and Phe195 of the $\gamma 134.5$ protein of herpes simplex virus 1 are required for viral resistance to interferon- $\alpha / \beta$. Virology 2001, 290, 115-120. [CrossRef] [PubMed]

325. Leib, D.A.; Machalek, M.A.; Williams, B.R.G.; Silverman, R.H.; Virgin, H.W. Specific phenotypic restoration of an attenuated virus by knockout of a host resistance gene. Proc. Natl. Acad. Sci. USA 2000, 97, 6097-6101. [CrossRef] [PubMed]

326. Verpooten, D.; Ma, Y.; Hou, S.; Yan, Z.; He, B. Control of TANK-binding kinase 1-mediated signaling by the gamma(1)34.5 protein of herpes simplex virus 1. J. Biol. Chem. 2009, 284, 1097-1105. [CrossRef] [PubMed]

327. Manivanh, R.; Mehrbach, J.; Knipe, D.M.; Leib, D.A. Role of herpes simplex virus $1 \gamma 34.5$ in the regulation of IRF3 signaling. J. Virol. 2017, 91. [CrossRef]

328. Orvedahl, A.; Alexander, D.; Tallóczy, Z.; Sun, Q.; Wei, Y.; Zhang, W.; Burns, D.; Leib, D.A.; Levine, B. HSV-1 ICP34.5 confers neurovirulence by targeting the Beclin 1 autophagy protein. Cell Host Microbe 2007, 1, 23-35. [CrossRef]

329. Tallóczy, Z.; Jiang, W.; Virgin, H.W.; Leib, D.A.; Scheuner, D.; Kaufman, R.J.; Eskelinen, E.-L.; Levine, B. Regulation of starvationand virus-induced autophagy by the eIF2 $\alpha$ kinase signaling pathway. Proc. Natl. Acad. Sci. USA 2002, 99, 190-195. [CrossRef]

330. Leib, D.A.; Alexander, D.E.; Cox, D.; Yin, J.; Ferguson, T.A. Interaction of ICP34.5 with Beclin 1 modulates herpes simplex virus type 1 pathogenesis through control of CD4+ T-cell responses. J. Virol. 2009, 83, 12164-12171. [CrossRef]

331. English, L.; Chemali, M.; Duron, J.; Rondeau, C.; Laplante, A.; Gingras, D.; Alexander, D.; Leib, D.; Norbury, C.; Lippé, R.; et al Autophagy enhances the presentation of endogenous viral antigens on MHC class I molecules during HSV-1 infection. Nat. Immunol. 2009, 10, 480-487. [CrossRef]

332. Gobeil, P.A.M.; Leib, D.A. Herpes simplex virus $\gamma 34.5$ interferes with autophagosome maturation and antigen presentation in dendritic cells. mBio 2012, 3. [CrossRef]

333. Alexander, D.E.; Ward, S.L.; Mizushima, N.; Levine, B.; Leib, D.A. Analysis of the role of autophagy in replication of herpes simplex virus in cell culture. J. Virol. 2007, 81, 12128-12134. [CrossRef] [PubMed]

334. Alexander, D.E.; Leib, D.A. Xenophagy in herpes simplex virus replication and pathogenesis. Autophagy 2008, 4, 101-103. [CrossRef] [PubMed]

335. Santana, S.; Bullido, M.J.; Recuero, M.; Valdivieso, F.; Aldudo, J. Herpes simplex virus type I induces an incomplete autophagic response in human neuroblastoma cells. J. Alzheimers Dis. 2012, 30, 815-831. [CrossRef] [PubMed]

336. Rasmussen, S.B.; Horan, K.A.; Holm, C.K.; Stranks, A.J.; Mettenleiter, T.C.; Simon, A.K.; Jensen, S.B.; Rixon, F.J.; He, B.; Paludan, S.R. Activation of autophagy by $\alpha$-herpesviruses in myeloid cells is mediated by cytoplasmic viral DNA through a mechanism dependent on stimulator of IFN genes. J. Immunol. 2011, 187, 5268-5276. [CrossRef]

337. Budida, R.; Stankov, M.V.; Döhner, K.; Buch, A.; Panayotova-Dimitrova, D.; Tappe, K.A.; Pohlmann, A.; Sodeik, B.; Behrens, G.M.N. Herpes simplex virus 1 interferes with autophagy of murine dendritic cells and impairs their ability to stimulate CD8(+) T lymphocytes. Eur. J. Immunol. 2017, 47, 1819-1834. [CrossRef]

338. York, I.A.; Roop, C.; Andrews, D.W.; Riddell, S.R.; Graham, F.L.; Johnson, D.C. A cytosolic herpes simplex virus protein inhibits antigen presentation to CD8+ T lymphocytes. Cell 1994, 77, 525-535. [CrossRef]

339. Banks, T.A.; Nair, S.; Rouse, B.T. Recognition by and in vitro induction of cytotoxic T lymphocytes against predicted epitopes of the immediate-early protein ICP27 of herpes simplex virus. J. Virol. 1993, 67, 613-616. [CrossRef]

340. Fruh, K.; Ahn, K.; Djaballah, H.; Sempe, P.; van Endert, P.M.; Tampé, R.; Peterson, P.A.; Yang, Y. A viral inhibitor of peptide transporters for antigen presentation. Nature 1995, 375, 415-418. [CrossRef]

341. Tomazin, R.; Hill, A.B.; Jugovic, P.; York, I.; van Endert, P.; Ploegh, H.L.; Andrews, D.W.; Johnson, D.C. Stable binding of the herpes simplex virus ICP47 protein to the peptide binding site of TAP. EMBO J. 1996, 15, 3256-3266. [CrossRef] 
342. Galocha, B.; Hill, A.; Barnett, B.C.; Dolan, A.; Raimondi, A.; Cook, R.F.; Brunner, J.; Mcgeoch, D.J.; Ploegh, H.L. The active site of ICP47, a herpes simplex virus-encoded inhibitor of the major histocompatibility complex (MHC)-encoded peptide transported associated with antigen processing (TAP), maps to the NH2-terminal 35 residues. J. Exp. Med. 1997, 185, 1565-1572. [CrossRef]

343. Ahn, K.; Meyer, T.H.; Uebel, S.; Sempé, P.; Djaballah, H.; Yang, Y.; Peterson, P.A.; Früh, K.; Tampé, R. Molecular mechanism and species specificity of TAP inhibition by herpes simplex virus protein ICP47. EMBO J. 1996, 15, 3247-3255. [CrossRef]

344. Tomazin, R.; van Schoot, N.E.G.; Goldsmith, K.; Jugovic, P.; Sempé, P.; Früh, K.; Johnson, D.C. Herpes simplex virus type 2 ICP47 inhibits human TAP but not mouse TAP. J. Virol. 1998, 72, 2560-2563. [CrossRef]

345. Jugovic, P.; Hill, A.M.; Tomazin, R.; Ploegh, H.; Johnson, D.C. Inhibition of major histocompatibility complex class I antigen presentation in pig and primate cells by herpes simplex virus type 1 and 2 ICP47. J. Virol. 1998, 72, 5076-5084. [CrossRef] [PubMed]

346. Orr, M.T.; Edelmann, K.H.; Vieira, J.; Corey, L.; Raulet, D.H.; Wilson, C.B. Inhibition of MHC class I is a virulence factor in herpes simplex virus infection of mice. PLoS Pathog. 2005, 1, e7. [CrossRef] [PubMed]

347. Liu, B.L.; Robinson, M.; Han, Z.Q.; Branston, R.H.; English, C.; Reay, P.; McGrath, Y.; Thomas, S.K.; Thornton, M.; Bullock, P.; et al. ICP34.5 deleted herpes simplex virus with enhanced oncolytic, immune stimulating, and anti-tumour properties. Gene Ther. 2003, 10, 292-303. [CrossRef] [PubMed]

348. Thomas, S.; Kuncheria, L.; Roulstone, V.; Kyula, J.N.; Mansfield, D.; Bommareddy, P.K.; Smith, H.; Kaufman, H.L.; Harrington, K.J.; Coffin, R.S. Development of a new fusion-enhanced oncolytic immunotherapy platform based on herpes simplex virus type 1. J. Immunother. Cancer 2019, 7, 1-17. [CrossRef]

349. Saric, T.; Chang, S.C.; Hattori, A.; York, I.A.; Markant, S.; Rock, K.L.; Tsujimoto, M.; Goldberg, A.L. An IFN- $\gamma$-induced aminopeptidase in the ER, ERAP I, trims precursors to MHC class I-presented peptides. Nat. Immunol. 2002, 3, 1169-1176. [CrossRef]

350. Marijt, K.A.; van Hall, T. To TAP or not to TAP: Alternative peptides for immunotherapy of cancer. Curr. Opin. Immunol. 2020, 64, 15-19. [CrossRef]

351. Raafat, N.; Sadowski-Cron, C.; Mengus, C.; Heberer, M.; Spagnoli, G.C.; Zajac, P. Preventing vaccinia virus class-I epitopes presentation by HSV-ICP47 enhances the immunogenicity of a TAP-independent cancer vaccine epitope. Int. J. Cancer 2012, 131, 659-669. [CrossRef]

352. Nishiyama, Y.; Kurachi, R.; Daikoku, T.; Umene, K. The US 9, 10, 11, and 12 genes of herpes simplex virus type 1 are of no importance for its neurovirulence and latency in mice. Virology 1993, 194, 419-423. [CrossRef] [PubMed]

353. Goldsmith, B.K.; Chen, W.; Johnson, D.C.; Hendricks, R.L. Infected Cell Protein (ICP) 47 enhances herpes simpex virus neurovirulence by blocking the CD8 T cell response. J. Exp. Med. 1998, 187, 341-348. [CrossRef] [PubMed]

354. Burgos, J.S.; Serrano-Saiz, E.; Sastre, I.; Valdivieso, F. ICP47 mediates viral neuroinvasiveness by induction of TAP protein following intravenous inoculation of herpes simplex virus type 1 in mice. J. Neurovirol. 2006, 12, 420-427. [CrossRef] [PubMed]

355. Lindahl, T. DNA repair enzymes. Annu. Rev. Biochem. 1982, 51, 61-87. [CrossRef]

356. Worrad, D.M.; Caradonna, S. Identification of the coding sequence for herpes simplex virus uracil-DNA glycosylase. J. Virol. 1988, 62, 4774-4777. [CrossRef]

357. Savva, R.; Pearl, L.H. Crystallization and preliminary X-ray analysis of the uracil-DNA glycosylase DNA repair enzyme from herpes simplex virus type 1. J. Mol. Biol. 1993, 234, 910-912. [CrossRef]

358. Caradonna, S.J.; Cheng, Y.C. Induction of uracil-DNA glycosylase and dUTP nucleotidohydrolase activity in herpes simplex virus-infected human cells. J. Biol. Chem. 1981, 256, 9834-9837.

359. Caradonna, S.; Worrad, D.; Lirette, R. Isolation of a herpes simplex virus cDNA encoding the DNA repair enzyme uracil-DNA glycosylase. J. Virol. 1987, 61, 3040-3047. [CrossRef]

360. Focher, F.; Verri, A.; Verzeletti, S.; Mazzarello, P.; Spadari, S. Uracil in Oris of herpes simplex 1 alters its specific recognition by origin binding protein (OBP): Does virus induced uracil-DNA glycosylase play a key role in viral reactivation and replication? Chromosoma 1992, 102, 67-71. [CrossRef]

361. Pyles, R.B.; Thompson, R.L. Evidence that the herpes simplex virus type 1 uracil DNA glycosylase is required for efficient viral replication and latency in the murine nervous system. J. Virol. 1994, 68, 4963-4972. [CrossRef]

362. Bogani, F.; Chua, C.N.; Boehmer, P.E. Reconstitution of uracil DNA glycosylase-initiated base excision repair in herpes simplex virus-1. J. Biol. Chem. 2009, 284, 16784-16790. [CrossRef] [PubMed]

363. Bogani, F.; Corredeira, I.; Fernandez, V.; Sattler, U.; Rutvisuttinunt, W.; Defais, M.; Boehmer, P.E. Association between the herpes simplex virus-1 DNA polymerase and uracil DNA glycosylase. J. Biol. Chem. 2010, 285, 27664-27672. [CrossRef] [PubMed]

364. Prichard, M.N.; Lawlor, H.; Duke, G.M.; Mo, C.; Wang, Z.; Dixon, M.; Kemble, G.; Kern, E.R. Human cytomegalovirus uracil DNA glycosylase associates with ppUL44 and accelerates the accumulation of viral DNA. Virol. J. 2005, 2, 55. [CrossRef] [PubMed]

365. Courcelle, C.T.; Courcelle, J.; Prichard, M.N.; Mocarski, E.S. Requirement for uracil-DNA glycosylase during the transition to late-phase cytomegalovirus DNA replication. J. Virol. 2001, 75, 7592-7601. [CrossRef] [PubMed]

366. Li, M.; Zou, X.; Wang, Y.; Xu, Z.; Ou, X.; Li, Y.; Liu, D.; Guo, Y.; Deng, Y.; Jiang, S.; et al. The nuclear localization signal-mediated nuclear targeting of herpes simplex virus 1 early protein UL2 is important for efficient viral production. Aging 2020, 12, 2921-2938. [CrossRef] [PubMed]

367. Mullaney, J.; Moss, H.W.; McGeoch, D.J. Gene UL2 of herpes simplex virus type 1 encodes a uracil-DNA glycosylase. J. Gen. Virol. 1989, 70, 449-454. [CrossRef] [PubMed] 
368. Dean, H.J.; Cheung, A.K. A 3' coterminal gene cluster in pseudorabies virus contains herpes simplex virus UL1, UL2, and UL3 gene homologs and a unique UL3.5 open reading frame. J. Virol. 1993, 67, 5955-5961. [CrossRef]

369. Khattar, S.K.; van Drunen Littel-van den Hurk, S.; Babiuk, L.A.; Tikoo, S.K. Identification and transcriptional analysis of a 3'-coterminal gene cluster containing UL1, UL2, UL3, and UL3.5 open reading frames of bovine herpesvirus-1. Virology 1995, 213, 28-37. [CrossRef]

370. Li, H.; Liu, S.; Han, Z.; Shao, Y.; Chen, S.; Kong, X. Comparative analysis of the genes UL1 through UL7 of the duck enteritis virus and other herpesviruses of the subfamily Alphaherpesvirinae. Genet. Mol. Biol. 2009, 32, 121-128. [CrossRef]

371. Prichard, M.N.; Duke, G.M.; Mocarski, E.S. Human cytomegalovirus uracil DNA glycosylase is required for the normal temporal regulation of both DNA synthesis and viral replication. J. Virol. 1996, 70, 3018-3025. [CrossRef]

372. Puvion-Dutilleul, F.; Pichard, E. Viral alkaline nuclease in intranuclear dense bodies induced by herpes simplex infection. Biol. Cell 1986, 58, 15-22. [CrossRef] [PubMed]

373. Knipe, D.M. The role of viral and cellular nuclear proteins in herpes simplex virus replication. Adv. Virus Res. 1989, 37, 85-123. [PubMed]

374. Banks, L.M.; Halliburton, I.W.; Purifoy, D.J.; Killington, R.A.; Powell, K.L. Studies on the herpes simplex virus alkaline nuclease: Detection of type-common and type-specific epitopes on the enzyme. J. Gen. Virol. 1985, 66, 1-14. [CrossRef] [PubMed]

375. Daikoku, T.; Yamashita, Y.; Tsurumi, T.; Nishiyama, Y. The US3 protein kinase of herpes simplex virus type 2 is associated with phosphorylation of the UL12 alkaline nuclease in vitro. Arch. Virol. 1995, 140, 1637-1644. [CrossRef]

376. Costa, R.H.; Draper, K.G.; Banks, L.; Powell, K.L.; Cohen, G.; Eisenberg, R.; Wagner, E.K. High-resolution characterization of herpes simplex virus type 1 transcripts encoding alkaline exonuclease and a 50,000-dalton protein tentatively identified as a capsid protein. J. Virol. 1983, 48, 591-603. [CrossRef]

377. Preston, C.M.; Cordingley, M.G. mRNA- and DNA-directed synthesis of herpes simplex virus-coded exonuclease in Xenopus laevis oocytes. J. Virol. 1982, 43, 386-394. [CrossRef]

378. Wathen, M.W.; Hay, J. Physical mapping of the herpes simplex virus type 2 nuc- lesion affecting alkaline exonuclease activity by using herpes simplex virus type 1 deletion clones. J. Virol. 1984, 51, 237-241. [CrossRef]

379. Draper, K.G.; Devi-Rao, G.; Costa, R.H.; Blair, E.D.; Thompson, R.L.; Wagner, E.K. Characterization of the genes encoding herpes simplex virus type 1 and type 2 alkaline exonucleases and overlapping proteins. J. Virol. 1986, 57, 1023-1036. [CrossRef]

380. Baer, R.; Bankier, A.T.; Biggin, M.D.; Deininger, P.L.; Farrell, P.J.; Gibson, T.J.; Hatfull, G.; Hudson, G.S.; Satchwell, S.C.; Séguin, C. DNA sequence and expression of the B95-8 Epstein-Barr virus genome. Nature 1984, 310, 207-211. [CrossRef]

381. Grose, C.; Tyler, S.; Peters, G.; Hiebert, J.; Stephens, G.M.; Ruyechan, W.T.; Jackson, W.; Storlie, J.; Tipples, G.A. Complete DNA sequence analyses of the first two varicella-zoster virus glycoprotein E (D150N) mutant viruses found in North America: Evolution of genotypes with an accelerated cell spread phenotype. J. Virol. 2004, 78, 6799-6807. [CrossRef]

382. De Wind, N.; Peeters, B.P.; Zuderveld, A.; Gielkens, A.L.; Berns, A.J.; Kimman, T.G. Mutagenesis and characterization of a 41-kilobase-pair region of the pseudorabies virus genome: Transcription map, search for virulence genes, and comparison with homologs of herpes simplex virus type 1. Virology 1994, 200, 784-790. [CrossRef] [PubMed]

383. Gao, M.; Robertson, B.J.; McCann, P.J.; O’Boyle, D.R.; Weller, S.K.; Newcomb, W.W.; Brown, J.C.; Weinheimer, S.P. Functional conservations of the alkaline nuclease of herpes simplex type 1 and human cytomegalovirus. Virology 1998, 249, 460-470. [CrossRef] [PubMed]

384. Weller, S.K.; Seghatoleslami, M.R.; Shao, L.; Rowse, D.; Carmichael, E.P. The herpes simplex virus type 1 alkaline nuclease is not essential for viral DNA synthesis: Isolation and characterization of a lacZ insertion mutant. J. Gen. Virol. 1990, 71, $2941-2952$. [CrossRef] [PubMed]

385. Shao, L.; Rapp, L.M.; Weller, S.K. Herpes simplex virus 1 alkaline nuclease is required for efficient egress of capsids from the nucleus. Virology 1993, 196, 146-162. [CrossRef]

386. Sagou, K.; Uema, M.; Kawaguchi, Y. Nucleolin is required for efficient nuclear egress of herpes simplex virus type 1 nucleocapsids. J. Virol. 2010, 84, 2110-2121. [CrossRef]

387. Porter, I.M.; Stow, N.D. Virus particles produced by the herpes simplex virus type 1 alkaline nuclease null mutant ambUL12 contain abnormal genomes. J. Gen. Virol. 2004, 85, 583-591. [CrossRef]

388. Grady, L.M.; Szczepaniak, R.; Murelli, R.P.; Masaoka, T.; Le Grice, S.F.J.; Wright, D.L.; Weller, S.K. The exonuclease activity of herpes simplex virus 1 UL12 is required for production of viral DNA that can be packaged to produce infectious virus. J. Virol. 2017, 91. [CrossRef]

389. Martinez, R.; Sarisky, R.T.; Weber, P.C.; Weller, S.K. Herpes simplex virus type 1 alkaline nuclease is required for efficient processing of viral DNA replication intermediates. J. Virol. 1996, 70, 2075-2085. [CrossRef]

390. Goldstein, J.N.; Weller, S.K. In vitro processing of herpes simplex virus type 1 DNA replication intermediates by the viral alkaline nuclease, UL12. J. Virol. 1998, 72, 8772-8781. [CrossRef]

391. Henderson, J.O.; Ball-Goodrich, L.J.; Parris, D.S. Structure-function analysis of the herpes simplex virus type 1 UL12 gene: Correlation of deoxyribonuclease activityin vitrowith replication function. Virology 1998, 243, 247-259. [CrossRef]

392. Reuven, N.B.; Staire, A.E.; Myers, R.S.; Weller, S.K. The herpes simplex virus type 1 alkaline nuclease and single-stranded DNA binding protein mediate strand exchange in vitro. J. Virol. 2003, 77, 7425-7433. [CrossRef] [PubMed] 
393. Reuven, N.B.; Willcox, S.; Griffith, J.D.; Weller, S.K. Catalysis of strand exchange by the HSV-1 UL12 and ICP8 proteins: Potent ICP8 recombinase activity is revealed upon resection of dsDNA substrate by nuclease. J. Mol. Biol. 2004, 342, 57-71. [CrossRef] [PubMed]

394. Reuven, N.B.; Weller, S.K. Herpes simplex virus type 1 single-strand DNA binding protein ICP8 enhances the nuclease activity of the UL12 alkaline nuclease by increasing its processivity. J. Virol. 2005, 79, 9356-9358. [CrossRef] [PubMed]

395. Schumacher, A.J.; Mohni, K.N.; Kan, Y.; Hendrickson, E.A.; Stark, J.M.; Weller, S.K. The HSV-1 exonuclease, UL12, stimulates recombination by a single strand annealing mechanism. PLoS Pathog. 2012, 8, e1002862. [CrossRef] [PubMed]

396. Tolun, G.; Makhov, A.M.; Ludtke, S.J.; Griffith, J.D. Details of ssDNA annealing revealed by an HSV-1 ICP8-ssDNA binary complex. Nucleic Acids Res. 2013, 41, 5927-5937. [CrossRef] [PubMed]

397. Balasubramanian, N.; Bai, P.; Buchek, G.; Korza, G.; Weller, S.K. Physical interaction between the herpes simplex virus type 1 exonuclease, UL12, and the DNA double-strand break-sensing MRN complex. J. Virol. 2010, 84, 12504-12514. [CrossRef] [PubMed]

398. Bronstein, J.C.; Weller, S.K.; Weber, P.C. The product of the UL12.5 gene of herpes simplex virus type 1 is a capsid-associated nuclease. J. Virol. 1997, 71, 3039-3047. [CrossRef]

399. Reuven, N.B.; Antoku, S.; Weller, S.K. The UL12.5 gene product of herpes simplex virus type 1 exhibits nuclease and strand exchange activities but does not localize to the nucleus. J. Virol. 2004, 78, 4599-4608. [CrossRef]

400. Martinez, R.; Goldstein, J.N.; Weller, S.K. The product of the UL12.5 gene of herpes simplex virus type 1 is not essential for lytic viral growth and is not specifically associated with capsids. Virology 2002, 298, 248-257. [CrossRef]

401. Saffran, H.A.; Pare, J.M.; Corcoran, J.A.; Weller, S.K.; Smiley, J.R. Herpes simplex virus eliminates host mitochondrial DNA. EMBO Rep. 2007, 8, 188-193. [CrossRef]

402. Duguay, B.A.; Smiley, J.R. Mitochondrial nucleases ENDOG and EXOG participate in mitochondrial DNA depletion initiated by herpes simplex virus 1 UL12.5. J. Virol. 2013, 87, 11787-11797. [CrossRef] [PubMed]

403. Corcoran, J.A.; Saffran, H.A.; Duguay, B.A.; Smiley, J.R. Herpes simplex virus UL12.5 targets mitochondria through a mitochondrial localization sequence proximal to the $\mathrm{N}$ terminus. J. Virol. 2009, 83, 2601-2610. [CrossRef] [PubMed]

404. Duguay, B.A.; Saffran, H.A.; Ponomarev, A.; Duley, S.A.; Eaton, H.E.; Smiley, J.R. Elimination of mitochondrial DNA is not required for herpes simplex virus 1 replication. J. Virol. 2014, 88, 2967-2976. [CrossRef] [PubMed]

405. Björnberg, O.; Bergman, A.C.; Rosengren, A.M.; Persson, R.; Lehman, I.R.; Nyman, P.O. dUTPase from herpes simplex virus type 1; purification from infected green monkey kidney (Vero) cells and from an overproducing Escherichia coli strain. Protein Expr. Purif. 1993, 4, 149-159. [CrossRef] [PubMed]

406. Preston, V.G.; Fisher, F.B. Identification of the herpes simplex virus type 1 gene encoding the dUTPase. Virology 1984, 138, 58-68. [CrossRef]

407. Caradonna, S.J.; Adamkiewicz, D.M. Purification and properties of the deoxyuridine triphosphate nucleotidohydrolase enzyme derived from HeLa S3 cells. Comparison to a distinct dUTP nucleotidohydrolase induced in herpes simplex virus-infected HeLa S3 cells. J. Biol. Chem. 1984, 259, 5459-5464.

408. Williams, M. V Deoxyuridine triphosphate nucleotidohydrolase induced by herpes simplex virus type 1 . Purification and characterization of induced enzyme. J. Biol. Chem. 1984, 259, 10080-10084.

409. Pyles, R.B.; Sawtell, N.M.; Thompson, R.L. Herpes simplex virus type 1 dUTPase mutants are attenuated for neurovirulence, neuroinvasiveness, and reactivation from latency. J. Virol. 1992, 66, 6706-6713. [CrossRef]

410. Lirette, R.; Caradonna, S. Inhibition of phosphorylation of cellular dUTP nucleotidohydrolase as a consequence of herpes simplex virus infection. J. Cell. Biochem. 1990, 43, 339-353. [CrossRef]

411. Kato, A.; Tsuda, S.; Liu, Z.; Kozuka-Hata, H.; Oyama, M.; Kawaguchi, Y. Herpes simplex virus 1 protein kinase Us3 phosphorylates viral dUTPase and regulates its catalytic activity in infected cells. J. Virol. 2014, 88, 655-666. [CrossRef]

412. Kato, A.; Shindo, K.; Maruzuru, Y.; Kawaguchi, Y. Phosphorylation of a herpes simplex virus 1 dUTPase by a viral protein kinase, Us3, dictates viral pathogenicity in the central nervous system but not at the periphery. J. Virol. 2014, 88, 2775-2785. [CrossRef] [PubMed]

413. Kato, A.; Hirohata, Y.; Arii, J.; Kawaguchi, Y. Phosphorylation of herpes simplex virus 1 dUTPase upregulated viral dUTPase activity to compensate for low cellular dUTPase activity for efficient viral replication. J. Virol. 2014, 88, 7776-7785. [CrossRef] [PubMed]

414. Riggio, M.P.; Onions, D.E. DNA sequence of a gene cluster in the equine herpesvirus-4 genome which contains a newly identified herpesvirus gene encoding a membrane protein. Arch. Virol. 1993, 133, 171-178. [CrossRef] [PubMed]

415. Baumeister, J.; Klupp, B.G.; Mettenleiter, T.C. Pseudorabies virus and equine herpesvirus 1 share a nonessential gene which is absent in other herpesviruses and located adjacent to a highly conserved gene cluster. J. Virol. 1995, 69, 5560-5567. [CrossRef]

416. Williams, M.V.J. Demonstration of a herpes simplex virus type 2-induced deoxyuridine triphosphate nucleotidohydrolase in infected KB cells and in biochemically transformed HeLa cells. J. Gen. Virol. 1984, 65, 209-213. [CrossRef]

417. Liang, X.; Tang, M.; Manns, B.; Babiuk, L.A.; Zamb, T.J. Identification and deletion mutagenesis of the bovine herpesvirus 1 dUTPase gene and a gene homologous to herpes simplex virus UL49.5. Virology 1993, 195, 42-50. [CrossRef]

418. Ariza, M.-E.; Glaser, R.; Kaumaya, P.T.P.; Jones, C.; Williams, M. V The EBV-encoded dUTPase activates NF- $\mathrm{kB}$ through the TLR2 and MyD88-dependent signaling pathway. J. Immunol. 2009, 182, 851-859. [CrossRef] 
419. Glaser, R.; Litsky, M.L.; Padgett, D.A.; Baiocchi, R.A.; Yang, E.V.; Chen, M.; Yeh, P.-E.; Green-Church, K.B.; Caligiuri, M.A.; Williams, M. V EBV-encoded dUTPase induces immune dysregulation: Implications for the pathophysiology of EBV-associated disease. Virology 2006, 346, 205-218. [CrossRef]

420. Ariza, M.E.; Glaser, R.; Williams, M. V Human herpesviruses-encoded dUTPases: A family of proteins that modulate dendritic cell function and innate immunity. Front. Microbiol. 2014, 5, 504. [CrossRef]

421. Goldstein, D.J.; Weller, S.K. Factor(s) present in herpes simplex virus type 1-infected cells can compensate for the loss of the large subunit of the viral ribonucleotide reductase: Characterization of an ICP6 deletion mutant. Virology 1988, 166, 41-51. [CrossRef]

422. Bacchetti, S.; Evelegh, M.J.; Muirhead, B. Identification and separation of the two subunits of the herpes simplex virus ribonucleotide reductase. J. Virol. 1986, 57, 1177-1181. [CrossRef] [PubMed]

423. Ren, Y. Glycoprotein M and ESCRT in Herpes Simplex Virus Type 1 Assembly. Ph.D. Thesis, University of Cambridge, Cambridge, UK, 2011.

424. Paradis, H.; Gaudreau, P.; Brazeau, P.; Langelier, Y. Mechanism of inhibition of herpes simplex virus (HSV) ribonucleotide reductase by a nonapeptide corresponding to the carboxyl terminus of its subunit 2 . Specific binding of a photoaffinity analog, [4'-azido-Phe6]HSV H2-(6-15), to subunit 1. J. Biol. Chem. 1988, 263, 16045-16050. [PubMed]

425. Spector, T.; Stonehuerner, J.G.; Biron, K.K.; Averett, D.R. Ribonucleotide reductase induced by varicella zoster virus. Characterization, and potentiation of acyclovir by its inhibition. Biochem. Pharmacol. 1987, 36, 4341-4346. [CrossRef]

426. Coen, D.M.; Kosz-Vnenchak, M.; Jacobson, J.G.; Leib, D.A.; Bogard, C.L.; Schaffer, P.A.; Tyler, K.L.; Knipe, D.M. Thymidine kinase-negative herpes simplex virus mutants establish latency in mouse trigeminal ganglia but do not reactivate. Proc. Natl. Acad. Sci. USA 1989, 86, 4736-4740. [CrossRef]

427. Campadelli-Fiume, G.; De Giovanni, C.; Gatta, V.; Nanni, P.; Lollini, P.L.; Menotti, L. Rethinking herpes simplex virus: The way to oncolytic agents. Rev. Med. Virol. 2011, 21, 213-226. [CrossRef]

428. Gober, M.D.; Wales, S.Q.; Hunter, J.C.; Sharma, B.K.; Aurelian, L. Stress up-regulates neuronal expression of the herpes simplex virus type 2 large subunit of ribonucleotide reductase (R1; ICP10) by activating activator protein 1. J. Neurovirol. 2005, 11, 329-336. [CrossRef] [PubMed]

429. Langelier, Y.; Bergeron, S.; Chabaud, S.; Lippens, J.; Guilbault, C.; Sasseville, A.M.J.; Denis, S.; Mosser, D.D.; Massie, B. The R1 subunit of herpes simplex virus ribonucleotide reductase protects cells against apoptosis at, or upstream of, caspase- 8 activation. J. Gen. Virol. 2002, 83, 2779-2789. [CrossRef] [PubMed]

430. Dufour, F.; Sasseville, A.M.J.; Chabaud, S.; Massie, B.; Siegel, R.M.; Langelier, Y. The ribonucleotide reductase R1 subunits of herpes simplex virus types 1 and 2 protect cells against TNF $\alpha$ - and FasL-induced apoptosis by interacting with caspase-8. Apoptosis 2011, 16, 256-271. [CrossRef] [PubMed]

431. Brandt, C.R.; Kintner, R.L.; Pumfery, A.M.; Visalli, R.J.; Grau, D.R. The herpes simplex virus ribonucleotide reductase is required for ocular virulence. J. Gen. Virol. 1991, 72, 2043-2049. [CrossRef] [PubMed]

432. Turk, S.R.; Kik, N.A.; Birch, G.M.; Chiego, D.J., Jr.; Shipman, C., Jr. Herpes simplex virus type 1 ribonucleotide reductase null mutants induce lesions in guinea pigs. Virology 1989, 173, 733-735. [CrossRef]

433. Mostafa, H.H.; Thompson, T.W.; Konen, A.J.; Haenchen, S.D.; Hilliard, J.G.; Macdonald, S.J.; Morrison, L.A.; Davido, D.J. Herpes simplex virus 1 mutant with point mutations in UL39 Is impaired for acute viral replication in mice, establishment of latency, and explant-induced reactivation. J. Virol. 2018, 92, 1-12. [CrossRef] [PubMed]

434. Davido, D.J.; Tu, E.M.; Wang, H.; Korom, M.; Gazquez Casals, A.; Reddy, P.J.; Mostafa, H.H.; Combs, B.; Haenchen, S.D.; Morrison, L.A. Attenuated herpes simplex virus 1 (HSV-1) expressing a mutant form of ICP6 stimulates a strong immune response that protects mice against HSV-1-induced corneal disease. J. Virol. 2018, 92. [CrossRef] [PubMed]

435. Fenwick, M.L.; Clark, J. Early and delayed shut-off of host protein synthesis in cells infected with herpes simplex virus. J. Gen. Virol. 1982, 61, 121-125. [CrossRef] [PubMed]

436. Fenwick, M.L.; McMenamin, M.M. Early virion-associated suppression of cellular protein synthesis by herpes simplex virus is accompanied by inactivation of mRNA. J. Gen. Virol. 1984, 65, 1225-1228. [CrossRef]

437. Fenwick, M.L.; Walker, M.J. Suppression of the synthesis of cellular macromolecules by herpes simplex virus. J. Gen. Virol. 1978, 41, 37-51. [CrossRef]

438. Kwong, A.D.; Frenkel, N. Herpes simplex virus-infected cells contain a function(s) that destabilizes both host and viral mRNAs. Proc. Natl. Acad. Sci. USA 1987, 84, 1926-1930. [CrossRef]

439. Kwong, A.D.; Kruper, J.A.; Frenkel, N. Herpes simplex virus virion host shutoff function. J. Virol. 1988, 62, 912-921. [CrossRef]

440. Oroskar, A.A.; Read, G.S. Control of mRNA stability by the virion host shutoff function of herpes simplex virus. J. Virol. 1989, 63, 1897-1906. [CrossRef]

441. Read, G.S.; Frenkel, N. Herpes simplex virus mutants defective in the virion-associated shutoff of host polypeptide synthesis and exhibiting abnormal synthesis of alpha (immediate early) viral polypeptides. J. Virol. 1983, 46, 498-512. [CrossRef]

442. Schek, N.; Bachenheimer, S.L. Degradation of cellular mRNAs induced by a virion-associated factor during herpes simplex virus infection of Vero cells. J. Virol. 1985, 55, 601-610. [CrossRef]

443. Berthomme, H.; Jacquemont, B.; Epstein, A. The pseudorabies virus host-shutoff homolog gene: Nucleotide sequence and comparison with alphaherpesvirus protein counterparts. Virology 1993, 193, 1028-1032. [CrossRef] [PubMed]

444. Lu, P.; Jones, F.E.; Saffran, H.A.; Smiley, J.R. Herpes simplex virus virion host shutoff protein requires a mammalian factor for efficient in vitro endoribonuclease activity. J. Virol. 2001, 75, 1172-1185. [CrossRef] [PubMed] 
445. Smith, T.J.; Morrison, L.A.; Leib, D.A. Pathogenesis of herpes simplex virus type 2 virion host shutoff (vhs) mutants. J. Virol. 2002, 76, 2054-2061. [CrossRef] [PubMed]

446. Strelow, L.I.; Leib, D.A. Role of the virion host shutoff (vhs) of herpes simplex virus type 1 in latency and pathogenesis. J. Virol. 1995, 69, 6779-6786. [CrossRef]

447. Smibert, C.A.; Smiley, J.R. Differential regulation of endogenous and transduced beta-globin genes during infection of erythroid cells with a herpes simplex virus type 1 recombinant. J. Virol. 1990, 64, 3882-3894. [CrossRef]

448. Strelow, L.; Smith, T.; Leib, D. The virion host shutoff function of herpes simplex virus type 1 plays a role in corneal invasion and functions independently of the cell cycle. Virology 1997, 231, 28-34. [CrossRef]

449. Strelow, L.I.; Leib, D.A. Analysis of conserved domains of UL41 of herpes simplex virus type 1 in virion host shutoff and pathogenesis. J. Virol. 1996, 70, 5665-5667. [CrossRef]

450. Fenwick, M.L.; Everett, R.D. Inactivation of the shutoff gene (UL41) of herpes simplex virus types 1 and 2. J. Gen. Virol. 1990, 71, 2961-2967. [CrossRef]

451. Strom, T.; Frenkel, N. Effects of herpes simplex virus on mRNA stability. J. Virol. 1987, 61, 2198-2207. [CrossRef]

452. Krikorian, C.R.; Read, G.S. In vitro mRNA degradation system to study the virion host shutoff function of herpes simplex virus. J. Virol. 1991, 65, 112-122. [CrossRef]

453. Oroskar, A.A.; Read, G.S. A mutant of herpes simplex virus type 1 exhibits increased stability of immediate-early (alpha) mRNAs. J. Virol. 1987, 61, 604-606. [CrossRef] [PubMed]

454. Lam, Q.; Smibert, C.A.; Koop, K.E.; Lavery, C.; Capone, J.P.; Weinheimer, S.P.; Smiley, J.R. Herpes simplex virus VP16 rescues viral mRNA from destruction by the virion host shutoff function. EMBO J. 1996, 15, 2575-2581. [CrossRef] [PubMed]

455. Taddeo, B.; Sciortino, M.T.; Zhang, W.; Roizman, B. Interaction of herpes simplex virus RNase with VP16 and VP22 is required for the accumulation of the protein but not for accumulation of mRNA. Proc. Natl. Acad. Sci. USA 2007, 104, 12163-12168. [CrossRef] [PubMed]

456. Taddeo, B.; Zhang, W.; Roizman, B. The herpes simplex virus host shutoff RNase degrades cellular and viral mRNAs made before infection but not viral mRNA made after infection. J. Virol. 2013, 87, 4516-4522. [CrossRef] [PubMed]

457. Esclatine, A.; Taddeo, B.; Evans, L.; Roizman, B. The herpes simplex virus $1 U_{L} 41$ gene-dependent destabilization of cellular RNAs is selective and may be sequence-specific. Proc. Natl. Acad. Sci. USA 2004, 101, 3603-3608. [CrossRef] [PubMed]

458. Sorenson, C.M.; Hart, P.A.; Ross, J. Analysis of herpes simplex virus-induced mRNA destabilizing activity using an in vitro mRNA decay system. Nucleic Acids Res. 1991, 19, 4459-4465. [CrossRef] [PubMed]

459. Zelus, B.D.; Stewart, R.S.; Ross, J. The virion host shutoff protein of herpes simplex virus type 1: Messenger ribonucleolytic activity in vitro. J. Virol. 1996, 70, 2411-2419. [CrossRef]

460. David, N.E.; Feng, P.; Mian, I.S.; Read, G.S. mRNA Degradation by the virion host shutoff (vhs) protein of herpes simplex virus: Genetic and Biochemical evidence that vhs is a nuclease. J. Virol. 2002, 76, 8560-8571. [CrossRef]

461. Elgadi, M.M.; Hayes, C.E.; Smiley, J.R. The herpes simplex virus vhs protein induces endoribonucleolytic cleavage of target RNAs in cell extracts. J. Virol. 1999, 73, 7153-7164. [CrossRef]

462. Doherty, A.J.; Serpell, L.C.; Ponting, C.P. The helix-hairpin-helix DNA-binding motif: A structural basis for non-sequence-specific recognition of DNA. Nucleic Acids Res. 1996, 24, 2488-2497. [CrossRef]

463. Taddeo, B.; Roizman, B. The virion host shutoff protein (UL41) of herpes simplex virus 1 is an endoribonuclease with a substrate specificity similar to that of RNase A. J. Virol. 2006, 80, 9341-9345. [CrossRef] [PubMed]

464. Taddeo, B.; Esclatine, A.; Zhang, W.; Roizman, B. The stress-inducible immediate-early responsive gene IEX-1 is activated in cells infected with herpes simplex virus 1 , but several viral mechanisms, including $3^{\prime}$ degradation of its RNA, preclude expression of the gene. J. Virol. 2003, 77, 6178-6187. [CrossRef] [PubMed]

465. Esclatine, A.; Taddeo, B.; Roizman, B. Herpes simplex virus 1 induces cytoplasmic accumulation of TIA-1/TIAR and both synthesis and cytoplasmic accumulation of tristetraprolin, two cellular proteins that bind and destabilize AU-rich RNAs. J. Virol. 2004, 78, 8582-8592. [CrossRef] [PubMed]

466. Karr, B.M.; Read, G.S. The virion host shutoff function of herpes simplex virus degrades the $5^{\prime}$ end of a target mRNA before the $3^{\prime}$ end. Virology 1999, 264, 195-204. [CrossRef] [PubMed]

467. Perez-Parada, J.; Saffran, H.A.; Smiley, J.R. RNA degradation induced by the herpes simplex virus vhs protein proceeds $5^{\prime}$ to $3^{\prime}$ in vitro. J. Virol. 2004, 78, 13391-13394. [CrossRef] [PubMed]

468. Feng, P.; Everly, D.N., Jr.; Read, G.S. mRNA decay during herpesvirus infections: Interaction between a putative viral nuclease and a cellular translation factor. J. Virol. 2001, 75, 10272-10280. [CrossRef] [PubMed]

469. Feng, P.; Everly, D.N.J.; Read, G.S. mRNA decay during herpes simplex virus (HSV) infections: Protein-protein interactions involving the HSV virion host shutoff protein and translation factors eIF4H and eIF4A. J. Virol. 2005, 79, 9651-9664. [CrossRef]

470. Benetti, L.; Munger, J.; Roizman, B. The herpes simplex virus 1 US3 protein kinase blocks caspase-dependent double cleavage and activation of the proapoptotic protein BAD. J. Virol. 2003, 77, 6567-6573. [CrossRef]

471. Doepker, R.C.; Hsu, W.-L.; Saffran, H.A.; Smiley, J.R. Herpes simplex virus virion host shutoff protein is stimulated by translation initiation factors eIF4B and eIF4H. J. Virol. 2004, 78, 4684-4699. [CrossRef]

472. Barzilai, A.; Zivony-Elbom, I.; Sarid, R.; Noah, E.; Frenkel, N. The herpes simplex virus type 1 vhs-UL41 gene secures viral replication by temporarily evading apoptotic cellular response to infection: Vhs-UL41 activity might require interactions with elements of cellular mRNA degradation machinery. J. Virol. 2006, 80, 505-513. [CrossRef] 
473. Shiflett, L.A.; Read, G.S. mRNA decay during herpes simplex virus (HSV) infections: Mutations that affect translation of an mRNA influence the sites at which it is cleaved by the HSV virion host shutoff (Vhs) protein. J. Virol. 2013, 87, 94-109. [CrossRef] [PubMed]

474. Suzutani, T.; Nagamine, M.; Shibaki, T.; Ogasawara, M.; Yoshida, I.; Daikoku, T.; Nishiyama, Y.; Azuma, M. The role of the UL41 gene of herpes simplex virus type 1 in evasion of non-specific host defence mechanisms during primary infection. J. Gen. Virol. 2000, 81, 1763-1771. [CrossRef] [PubMed]

475. Samady, L.; Costigliola, E.; MacCormac, L.; McGrath, Y.; Cleverley, S.; Lilley, C.E.; Smith, J.; Latchman, D.S.; Chain, B.; Coffin, R.S. Deletion of the virion host shutoff protein (vhs) from herpes simplex virus (hsv) relieves the viral block to dendritic cell activation: Potential of vhs- HSV vectors for dendritic cell-mediated immunotherapy. J. Virol. 2003, 77, 3768-3776. [CrossRef] [PubMed]

476. Cotter, C.R.; Nguyen, M.L.; Yount, J.S.; López, C.B.; Blaho, J.A.; Moran, T.M. The virion host shut-off (vhs) protein blocks a TLR-independent pathway of herpes simplex virus type 1 recognition in human and mouse dendritic cells. PLoS ONE 2010, 5, e8684. [CrossRef]

477. Eisemann, J.; Mühl-Zürbes, P.; Steinkasserer, A.; Kummer, M. Infection of mature dendritic cells with herpes simplex virus type 1 interferes with the interferon signaling pathway. Immunobiology 2007, 212, 877-886. [CrossRef]

478. Pasieka, T.J.; Cilloniz, C.; Lu, B.; Teal, T.H.; Proll, S.C.; Katze, M.G.; Leib, D.A. Host responses to wild-type and attenuated herpes simplex virus infection in the absence of Stat1. J. Virol. 2009, 83, 2075-2087. [CrossRef]

479. Burgess, H.M.; Mohr, I. Defining the role of stress granules in innate immune suppression by the herpes simplex virus 1 endoribonuclease VHS. J. Virol. 2018, 92. [CrossRef]

480. Dauber, B.; Poon, D.; dos Santos, T.; Duguay, B.A.; Mehta, N.; Saffran, H.A.; Smiley, J.R. The herpes simplex virus virion host shutoff protein enhances translation of viral true late mRNAs independently of suppressing protein kinase R and stress granule formation. J. Virol. 2016, 90, 6049-6057. [CrossRef]

481. Sciortino, M.T.; Parisi, T.; Siracusano, G.; Mastino, A.; Taddeo, B.; Roizman, B. The virion host shutoff RNase plays a key role in blocking the activation of protein kinase R in cells infected with herpes simplex virus 1. J. Virol. 2013, 87, 3271-3276. [CrossRef]

482. Dauber, B.; Saffran, H.A.; Smiley, J.R. The herpes simplex virus 1 virion host shutoff protein enhances translation of viral late mRNAs by preventing mRNA overload. J. Virol. 2014, 88, 9624-9632. [CrossRef]

483. Yao, X.-D.; Rosenthal, K.L. Herpes simplex virus type 2 virion host shutoff protein suppresses innate dsRNA antiviral pathways in human vaginal epithelial cells. J. Gen. Virol. 2011, 92, 1981-1993. [CrossRef] [PubMed]

484. Kit, S.; Dubbs, D.R.; Piekarski, L.J.; Hsu, T.C. Deletion of thymidine kinase activity from L cells resistant to bromodeoxyuridine. Exp. Cell Res. 1963, 31, 297-312. [CrossRef]

485. Deville-Bonne, D.; el Amri, C.; Meyer, P.; Chen, Y.; Agrofoglio, L.A.; Janin, J. Human and viral nucleoside/nucleotide kinases involved in antiviral drug activation: Structural and catalytic properties. Antiviral Res. 2010, 86, 101-120. [CrossRef] [PubMed]

486. McKnight, S.L. The nucleotide sequence and transcript map of the herpes simplex virus thymidine kinase gene. Nucleic Acids Res. 1980, 8, 5949-5964. [CrossRef]

487. Liu, Q.; Summers, W.C. Site-directed mutagenesis of a nucleotide-binding domain in HSV-1 thymidine kinase: Effects on catalytic activity. Virology 1988, 163, 638-642. [CrossRef]

488. Gentry, G.A. Viral thymidine kinases and their relatives. Pharmacol. Ther. 1992, 54, 319-355. [CrossRef]

489. Wild, K.; Bohner, T.; Folkers, G.; Schulz, G.E. The structures of thymidine kinase from Herpes simplex virus type 1 in complex with substrates and a substrate analogue. Protein Sci. 1997, 6, 2097-2106. [CrossRef]

490. Elion, G.B.; Furman, P.A.; Fyfe, J.A.; de Miranda, P.; Beauchamp, L.; Schaeffer, H.J. Selectivity of action of an antiherpetic agent, 9-(2-hydroxyethoxymethyl) guanine. Proc. Natl. Acad. Sci. USA 1977, 74, 5716-5720. [CrossRef]

491. Fyfe, J.A.; Keller, P.M.; Furman, P.A.; Miller, R.L.; Elion, G.B. Thymidine kinase from herpes simplex virus phosphorylates the new antiviral compound, 9-(2-hydroxyethoxymethyl)guanine. J. Biol. Chem. 1978, 253, 8721-8727.

492. Gustafson, E.A.; Chillemi, A.C.; Sage, D.R.; Fingeroth, J.D. The Epstein-Barr virus thymidine kinase does not phosphorylate ganciclovir or acyclovir and demonstrates a narrow substrate specificity compared to the herpes simplex virus type 1 thymidine kinase. Antimicrob. Agents Chemother. 1998, 42, 2923-2931. [CrossRef]

493. Gustafson, E.A.; Schinazi, R.F.; Fingeroth, J.D. Human herpesvirus 8 open reading frame 21 is a thymidine and thymidylate kinase of narrow substrate specificity that efficiently phosphorylates zidovudine but not ganciclovir. J. Virol. 2000, 74, 684-692. [CrossRef] [PubMed]

494. Lock, M.J.; Thorley, N.; Teo, J.; Emery, V.C. Azidodeoxythymidine and didehydrodeoxythymidine as inhibitors and substrates of the human herpesvirus 8 thymidine kinase. J. Antimicrob. Chemother. 2002, 49, 359-366. [CrossRef] [PubMed]

495. Solaroli, N.; Johansson, M.; Balzarini, J.; Karlsson, A. Substrate specificity of three viral thymidine kinases (TK): Vaccinia virus TK, feline herpesvirus TK, and canine herpesvirus TK. Nucleosides Nucleotides Nucleic Acids 2006, 25, 1189-1192. [CrossRef] [PubMed]

496. Ehlers, B.; Dural, G.; Marschall, M.; Schregel, V.; Goltz, M.; Hentschke, J. Endotheliotropic elephant herpesvirus, the first betaherpesvirus with a thymidine kinase gene. J. Gen. Virol. 2006, 87, 2781-2789. [CrossRef]

497. Mittal, S.K.; Field, H.J. Analysis of the bovine herpesvirus type 1 thymidine kinase (TK) gene from wild-type virus and TKdeficient mutants. J. Gen. Virol. 1989, 70, 901-918. [CrossRef]

498. Dong, J.; Bai, J.; Sun, T.; Gu, Z.; Wang, J.; Sun, H.; Jiang, P. Comparative pathogenicity and immunogenicity of triple and double gene-deletion pseudorabies virus vaccine candidates. Res. Vet. Sci. 2017, 115, 17-23. [CrossRef] 
499. Pellicer, A.; Wigler, M.; Axel, R.; Silverstein, S. The transfer and stable integration of the HSV thymidine kinase gene into mouse cells. Cell 1978, 14, 133-141. [CrossRef]

500. Field, H.J.; Darby, G. Pathogenicity in mice of strains of herpes simplex virus which are resistant to acyclovir in vitro and in vivo. Antimicrob. Agents Chemother. 1980, 17, 209-216. [CrossRef]

501. Field, H.J.; Wildy, P. The pathogenicity of thymidine kinase-deficient mutants of herpes simplex virus in mice. J. Hyg. 1978, 81 , 267-277. [CrossRef]

502. Gordon, Y.J.; Gilden, D.M.; Becker, Y. HSV-1 thymidine kinase promotes virulence and latency in the mouse. Investig. Ophthalmol. Vis. Sci. 1983, 24, 599-602.

503. Price, R.W.; Khan, A. Resistance of peripheral autonomic neurons to in vivo productive infection by herpes simplex virus mutants deficient in thymidine kinase activity. Infect. Immun. 1981, 34, 571-580. [CrossRef] [PubMed]

504. Chen, S.H.; Cook, W.J.; Grove, K.L.; Coen, D.M. Human thymidine kinase can functionally replace herpes simplex virus type 1 thymidine kinase for viral replication in mouse sensory ganglia and reactivation from latency upon explant. J. Virol. 1998, 72, 6710-6715. [CrossRef] [PubMed]

505. Chen, S.-H.; Lin, Y.-W.; Griffiths, A.; Huang, W.-Y.; Chen, S.-H. Competition and complementation between thymidine kinasenegative and wild-type herpes simplex virus during co-infection of mouse trigeminal ganglia. J. Gen. Virol. 2006, 87, 3495-3502. [CrossRef] [PubMed]

506. Leist, T.P.; Sandri-Goldin, R.M.; Stevens, J.G. Latent infections in spinal ganglia with thymidine kinase-deficient herpes simplex virus. J. Virol. 1989, 63, 4976-4978. [CrossRef] [PubMed]

507. Chen, S.-H.; Pearson, A.; Coen, D.M.; Chen, S.-H. Failure of thymidine kinase-negative herpes simplex virus to reactivate from latency following efficient establishment. J. Virol. 2004, 78, 520-523. [CrossRef] [PubMed]

508. Huang, C.-Y.; Yao, H.-W.; Wang, L.-C.; Shen, F.-H.; Hsu, S.-M.; Chen, S.-H. Thymidine kinase-negative herpes simplex virus 1 can efficiently establish persistent infection in neural tissues of nude mice. J. Virol. 2017, 91. [CrossRef] [PubMed]

509. Stroop, W.G.; Banks, M.C.; Qavi, H.; Chodosh, J.; Brown, S.M. A thymidine kinase deficient HSV-2 strain causes acute keratitis and establishes trigeminal ganglionic latency, but poorly reactivates in vivo. J. Med. Virol. 1994, 43, 297-309. [CrossRef]

510. Jacobson, J.G.; Ruffner, K.L.; Kosz-Vnenchak, M.; Hwang, C.B.; Wobbe, K.K.; Knipe, D.M.; Coen, D.M. Herpes simplex virus thymidine kinase and specific stages of latency in murine trigeminal ganglia. J. Virol. 1993, 67, 6903-6908. [CrossRef]

511. Tenser, R.B.; Hay, K.A.; Edris, W.A. Latency-associated transcript but not reactivatable virus is present in sensory ganglion neurons after inoculation of thymidine kinase-negative mutants of herpes simplex virus type 1. J. Virol. 1989, 63, 2861-2865. [CrossRef]

512. Field, H.J.; Bell, S.E.; Elion, G.B.; Nash, A.A.; Wildy, P. Effect of acycloguanosine treatment of acute and latent herpes simplex infections in mice. Antimicrob. Agents Chemother. 1979, 15, 554-561. [CrossRef]

513. Wilcox, C.L.; Crnic, L.S.; Pizer, L.I. Replication, latent infection, and reactivation in neuronal culture with a herpes simplex virus thymidine kinase-negative mutant. Virology 1992, 187, 348-352. [CrossRef]

514. Morfin, F.; Thouvenot, D. Herpes simplex virus resistance to antiviral drugs. J. Clin. Virol. 2003, 26, 29-37. [CrossRef]

515. Bacon, T.H.; Levin, M.J.; Leary, J.J.; Sarisky, R.T.; Sutton, D. Herpes simplex virus resistance to acyclovir and penciclovir after two decades of antiviral therapy. Clin. Microbiol. Rev. 2003, 16, 114-128. [CrossRef] [PubMed]

516. Coen, D.M.; Schaffer, P.A. Antiherpesvirus drugs: A promising spectrum of new drugs and drug targets. Nat. Rev. Drug Discov. 2003, 2, 278-288. [CrossRef] [PubMed]

517. Safrin, S. Treatment of acyclovir-resistant herpes simplex virus infections in patients with AIDS. J. Acquir. Immune Defic. Syndr. 1992, 5, 29-32.

518. Chen, H.; Beardsley, G.P.; Coen, D.M. Mechanism of ganciclovir-induced chain termination revealed by resistant viral polymerase mutants with reduced exonuclease activity. Proc. Natl. Acad. Sci. USA 2014, 111, 17462-17467. [CrossRef]

519. Zeng, Z.-J.; Xiang, S.-G.; Xue, W.-W.; Li, H.-D.; Ma, N.; Ren, Z.-J.; Xu, Z.-J.; Jiao, C.-H.; Wang, C.-Y.; Hu, W.-X. The cell death and DNA damages caused by the Tet-On regulating HSV-tk/GCV suicide gene system in MCF-7 cells. Biomed. Pharmacother. 2014, 68, 887-892. [CrossRef]

520. Osaki, T.; Tanio, Y.; Tachibana, I.; Hosoe, S.; Kumagai, T.; Kawase, I.; Oikawa, S.; Kishimoto, T. Gene therapy for carcinoembryonic antigen-producing human lung cancer cells by cell type-specific expression of herpes simplex virus thymidine kinase gene. Cancer Res. 1994, 54, 5258-5261.

521. Wei, S.-J.; Chao, Y.; Hung, Y.-M.; Lin, W.; Yang, D.-M.; Shih, Y.-L.; Ch'ang, L.-Y.; Whang-Peng, J.; Yang, W.K. S- and G2-phase cell cycle arrests and apoptosis induced by ganciclovir in murine melanoma cells transduced with herpes simplex virus thymidine kinase. Exp. Cell Res. 1998, 241, 66-75. [CrossRef]

522. Tomicic, M.T.; Thust, R.; Kaina, B. Ganciclovir-induced apoptosis in HSV-1 thymidine kinase expressing cells: Critical role of DNA breaks, Bcl-2 decline and caspase-9 activation. Oncogene 2002, 21, 2141-2153. [CrossRef]

523. Yin, X.; Yu, B.; Tang, Z.; He, B.; Ren, J.; Xiao, X.; Tang, W. Bifidobacterium infantis-mediated HSV-TK/GCV suicide gene therapy induces both extrinsic and intrinsic apoptosis in a rat model of bladder cancer. Cancer Gene Ther. 2013, 20, 77-81. [CrossRef] [PubMed]

524. Qiu, Y.; Peng, G.-L.; Liu, Q.-C.; Li, F.-L.; Zou, X.-S.; He, J.-X. Selective killing of lung cancer cells using carcinoembryonic antigen promoter and double suicide genes, thymidine kinase and cytosine deaminase (pCEA-TK/CD). Cancer Lett. 2012, 316, 31-38. [CrossRef] [PubMed] 
525. Beltinger, C.; Fulda, S.; Kammertoens, T.; Meyer, E.; Uckert, W.; Debatin, K.-M. Herpes simplex virus thymidine kinase/ganciclovir-induced apoptosis involves ligand-independent death receptor aggregation and activation of caspases. Proc. Natl. Acad. Sci. USA 1999, 96, 8699-8704. [CrossRef] [PubMed]

526. Beltinger, C.; Fulda, S.; Kammertoens, T.; Uckert, W.; Debatin, K.-M. Mitochondrial amplification of death signals determines thymidine kinase/ganciclovir-triggered activation of apoptosis. Cancer Res. 2000, 60, 3212-3217.

527. Liu, X.; Wang, S.; Guo, X.; Wei, F.; Yin, J.; Zang, Y.; Li, N.; Chen, D. Exogenous p53 and ASPP2 expression enhances rAdV-TK/ GCV-induced death in hepatocellular carcinoma cells lacking functional p53. Oncotarget 2016, 7, 18896-18905. [CrossRef]

528. Moolten, F.L.; Wells, J.M. Curability of tumors bearing herpes thymidine kinase genes transfered by retroviral vectors. J. Natl. Cancer Inst. 1990, 82, 297-300. [CrossRef]

529. Ram, Z.; Culver, K.W.; Walbridge, S.; Blaese, R.M.; Oldfield, E.H. In situ retroviral-mediated gene transfer for the treatment of brain tumors in rats. Cancer Res. 1993, 53, 83-88.

530. O'Malley, B.W.; Chen, S.-H.; Schwartz, M.R.; Woo, S.L.C. Adenovirus-mediated gene therapy for human head and neck squamous cell cancer in a nude mouse model. Cancer Res. 1995, 55, 1080-1085.

531. Hall, S.J.; Sanford, M.A.; Atkinson, G.; Chen, S.-H. Induction of potent antitumor natural killer cell activity by herpes simplex virus-thymidine kinase and ganciclovir therapy in an orthotopic mouse model of prostate cancer. Cancer Res. 1998, 58, 3221-3225.

532. Ram, Z.; Walbridge, S.; Shawker, T.; Culver, K.W.; Blaese, R.M.; Oldfield, E.H. The effect of thymidine kinase transduction and ganciclovir therapy on tumor vasculature and growth of 9L gliomas in rats. J. Neurosurg. 1994, 81, 256-260. [CrossRef]

533. Freeman, S.M.; Abboud, C.N.; Whartenby, K.A.; Packman, C.H.; Koeplin, D.S.; Moolten, F.L.; Abraham, G.N. The “Bystander Effect": Tumor regression when a fraction of the tumor mass is genetically modified. Cancer Res. 1993, 53, 5274-5283. [PubMed]

534. Hamel, W.; Magnelli, L.; Chiarugi, V.P.; Israel, M.A. Herpes simplex virus thymidine kinase/ganciclovir-mediated apoptotic death of bystander cells. Cancer Res. 1996, 56, 2697-2702. [PubMed]

535. Vile, R.G.; Nelson, J.A.; Castleden, S.; Chong, H.; Hart, I.R. Systemic gene therapy of murine melanoma using tissue specific expression of the HSVtk gene involves an immune component. Cancer Res. 1994, 54, 6228-6234. [PubMed]

536. Pastorakova, A.; Jakubechova, J.; Altanerova, U.; Altaner, C. Suicide gene therapy mediated with exosomes produced by mesenchymal stem/stromal cells stably transduced with HSV thymidine kinase. Cancers 2020, 12, 1096. [CrossRef]

537. Tamura, R.; Miyoshi, H.; Morimoto, Y.; Oishi, Y.; Sampetrean, O.; Iwasawa, C.; Mine, Y.; Saya, H.; Yoshida, K.; Okano, H.; et al. Gene therapy using neural stem/progenitor cells derived from human induced pluripotent stem cells: Visualization of migration and bystander killing effect. Hum. Gene Ther. 2020, 31, 352-366. [CrossRef]

538. Voges, J.; Reszka, R.; Gossmann, A.; Dittmar, C.; Richter, R.; Garlip, G.; Kracht, L.; Coenen, H.H.; Sturm, V.; Wienhard, K.; et al. Imaging-guided convection-enhanced delivery and gene therapy of glioblastoma. Ann. Neurol. 2003, 54, 479-487. [CrossRef]

539. Li, N.; Zhou, J.; Weng, D.; Zhang, C.; Li, L.; Wang, B.; Song, Y.; He, Q.; Lin, D.; Chen, D.; et al. Adjuvant adenovirus-mediated delivery of herpes simplex virus thymidine kinase administration improves outcome of liver transplantation in patients with advanced hepatocellular carcinoma. Clin. Cancer Res. 2007, 13, 5847-5854. [CrossRef]

540. Nasu, Y.; Saika, T.; Ebara, S.; Kusaka, N.; Kaku, H.; Abarzua, F.; Manabe, D.; Thompson, T.C.; Kumon, H. Suicide gene therapy with adenoviral delivery of HSV-tK gene for patients with local recurrence of prostate cancer after hormonal therapy. Mol. Ther. 2007, 15, 834-840. [CrossRef]

541. Van Putten, E.H.P.; Dirven, C.M.F.; van den Bent, M.J.; Lamfers, M.L.M. Sitimagene ceradenovec: A gene-based drug for the treatment of operable high-grade glioma. Futur. Oncol. 2010, 6, 1691-1710. [CrossRef]

542. Sangro, B.; Mazzolini, G.; Ruiz, M.; Ruiz, J.; Quiroga, J.; Herrero, I.; Qian, C.; Benito, A.; Larrache, J.; Olagüe, C.; et al. A phase I clinical trial of thymidine kinase-based gene therapy in advanced hepatocellular carcinoma. Cancer Gene Ther. 2010, 17, 837-843. [CrossRef]

543. Chiocca, E.A.; Aguilar, L.K.; Bell, S.D.; Kaur, B.; Hardcastle, J.; Cavaliere, R.; McGregor, J.; Lo, S.; Ray-Chaudhuri, A.; Chakravarti, A.; et al. Phase IB study of gene-mediated cytotoxic immunotherapy adjuvant to up-front surgery and intensive timing radiation for malignant glioma. J. Clin. Oncol. 2011, 29, 3611-3619. [CrossRef] [PubMed]

544. Islam, S.M.B.U.; Hong, Y.M.; Ornella, M.S.C.; Ngabire, D.; Jang, H.; Cho, E.; Kim, E.-K.; Hale, J.J.J.; Kim, C.H.; Ahn, S.C.; et al. Engineering and preclinical evaluation of western reserve oncolytic vaccinia virus expressing A167Y mutant herpes simplex virus thymidine kinase. Biomedicines 2020, 8, 426. [CrossRef] [PubMed]

545. Hossain, J.A.; Ystaas, L.R.; Mrdalj, J.; Välk, K.; Riecken, K.; Fehse, B.; Bjerkvig, R.; Grønli, J.; Miletic, H. Lentiviral HSV-Tk.007mediated suicide gene therapy is not toxic for normal brain cells. J. Gene Med. 2016, 18, 234-243. [CrossRef] [PubMed]

546. Kenarkoohi, A.; Bamdad, T.; Soleimani, M.; Soleimanjahi, H.; Fallah, A.; Falahi, S. HSV-TK Expressing mesenchymal stem cells exert inhibitory effect on cervical cancer model. Int. J. Mol. Cell. Med. 2020, 9, 146-154. [CrossRef] [PubMed]

547. Jo, E.B.; Lee, H.; Lee, K.W.; Kim, S.J.; Hong, D.; Park, J.B. Complete regression of metastatic de-differentiated liposarcoma with engineered mesenchymal stromal cells with dTRAIL and HSV-TK. Am. J. Transl. Res. 2020, 12, 3993-4000. [PubMed]

548. Islam, S.M.B.U.; Lee, B.; Jiang, F.; Kim, E.-K.; Ahn, S.C.; Hwang, T.-H. Engineering and characterization of oncolytic vaccinia virus expressing truncated herpes simplex virus thymidine kinase. Cancers 2020, 12, 228. [CrossRef] [PubMed]

549. Dührsen, L.; Hartfuß, S.; Hirsch, D.; Geiger, S.; Maire, C.L.; Sedlacik, J.; Guenther, C.; Westphal, M.; Lamszus, K.; Hermann, F.G.; et al. Preclinical analysis of human mesenchymal stem cells: Tumor tropism and therapeutic efficiency of local HSV-TK suicide gene therapy in glioblastoma. Oncotarget 2019, 10, 6049-6061. [CrossRef] 
550. Li, H.; Du, H.; Zhang, G.; Wu, Y.; Qiu, P.; Liu, J.; Guo, J.; Liu, X.; Sun, L.; Du, B.; et al. Curcumin plays a synergistic role in combination with HSV-TK/GCV in inhibiting growth of murine B16 melanoma cells and melanoma xenografts. PeerJ 2019, 7, e7760. [CrossRef]

551. Sagou, K.; Imai, T.; Sagara, H.; Uema, M.; Kawaguchi, Y. Regulation of the catalytic activity of herpes simplex virus 1 protein kinase Us3 by Autophosphorylation and its role in pathogenesis. J. Virol. 2009, 83, 5773-5783. [CrossRef]

552. Morimoto, T.; Arii, J.; Tanaka, M.; Sata, T.; Akashi, H.; Yamada, M.; Nishiyama, Y.; Uema, M.; Kawaguchi, Y. Differences in the regulatory and functional effects of the Us3 protein kinase activities of herpes simplex virus 1 and 2. J. Virol. 2009, 83, 11624-11634. [CrossRef]

553. Wisner, T.W.; Wright, C.C.; Kato, A.; Kawaguchi, Y.; Mou, F.; Baines, J.D.; Roller, R.J.; Johnson, D.C. Herpesvirus gB-induced fusion between the virion envelope and outer nuclear membrane during virus egress is regulated by the viral US3 kinase. J. Virol. 2009, 83, 3115-3126. [CrossRef] [PubMed]

554. Wild, P.; Leisinger, S.; de Oliveira, A.P.; Doehner, J.; Schraner, E.M.; Fraevel, C.; Ackermann, M.; Kaech, A. Nuclear envelope impairment is facilitated by the herpes simplex virus 1 Us3 kinase. F1000Research 2019, 8, 198. [CrossRef] [PubMed]

555. Tobler, K.; Senn, C.; Schraner, E.M.; Ackermann, M.; Fraefel, C.; Wild, P. The herpes simplex virus 1 Us3 kinase is involved in assembly of membranes needed for viral envelopment and in distribution of glycoprotein K. F1000Research $2019,8,727$. [CrossRef] [PubMed]

556. Reynolds, A.E.; Wills, E.G.; Roller, R.J.; Ryckman, B.J.; Baines, J.D. Ultrastructural localization of the herpes simplex virus type 1 UL31, UL34, and US3 proteins suggests specific roles in primary envelopment and egress of nucleocapsids. J. Virol. 2002, 76, 8939-8952. [CrossRef] [PubMed]

557. Ryckman, B.J.; Roller, R.J. Herpes simplex virus type 1 primary envelopment: UL34 protein modification and the US3-UL34 catalytic relationship. J. Virol. 2004, 78, 399-412. [CrossRef] [PubMed]

558. Mou, F.; Forest, T.; Baines, J.D. US3 of herpes simplex virus type 1 encodes a promiscuous protein kinase that phosphorylates and alters localization of lamin A/C in infected cells. J. Virol. 2007, 81, 6459-6470. [CrossRef] [PubMed]

559. Bjerke, S.L.; Roller, R.J. Roles for herpes simplex virus type 1 UL34 and US3 proteins in disrupting the nuclear lamina during herpes simplex virus type 1 egress. Virology 2006, 347, 261-276. [CrossRef]

560. Mou, F.; Wills, E.; Baines, J.D. Phosphorylation of the U(L)31 protein of herpes simplex virus 1 by the U(S)3-encoded kinase regulates localization of the nuclear envelopment complex and egress of nucleocapsids. J. Virol. 2009, 83, 5181-5191. [CrossRef]

561. Purves, F.C.; Spector, D.; Roizman, B. The herpes simplex virus 1 protein kinase encoded by the US3 gene mediates posttranslational modification of the phosphoprotein encoded by the UL34 gene. J. Virol. 1991, 65, 5757-5764. [CrossRef]

562. Reynolds, A.E.; Ryckman, B.J.; Baines, J.D.; Zhou, Y.; Liang, L.; Roller, R.J. UL31 and UL34 proteins of herpes simplex virus type 1 form a complex that accumulates at the nuclear rim and is required for envelopment of nucleocapsids. J. Virol. 2001, 75, 8803-8817. [CrossRef]

563. Kato, A.; Liu, Z.; Minowa, A.; Imai, T.; Tanaka, M.; Sugimoto, K.; Nishiyama, Y.; Arii, J.; Kawaguchi, Y. Herpes simplex virus 1 protein kinase Us3 and major tegument protein UL47 reciprocally regulate their subcellular localization in infected cells. J. Virol. 2011, 85, 9599-9613. [CrossRef] [PubMed]

564. Leopardi, R.; van Sant, C.; Roizman, B. The herpes simplex virus 1 protein kinase Us3 is required for protection from apoptosis induced by the virus. Proc. Natl. Acad. Sci. USA 1997, 94, 7891-7896. [CrossRef] [PubMed]

565. Galvan, V.; Roizman, B. Herpes simplex virus 1 induces and blocks apoptosis at multiple steps during infection and protects cells from exogenous inducers in a cell-type-dependent manner. Proc. Natl. Acad. Sci. USA 1998, 95, 3931-3936. [CrossRef] [PubMed]

566. Munger, J.; Roizman, B. The Us3 protein kinase of herpes simplex virus 1 mediates the posttranslational modification of BAD and prevents BAD-induced programmed cell death in the absence of other viral proteins. Proc. Natl. Acad. Sci. USA 2001, 98, 10410-10415. [CrossRef]

567. Hagglund, R.; Munger, J.; Poon, A.P.W.; Roizman, B. U(S)3 protein kinase of herpes simplex virus 1 blocks caspase 3 activation induced by the products of $\mathrm{U}(\mathrm{S}) 1.5$ and $\mathrm{U}(\mathrm{L}) 13$ genes and modulates expression of transduced $\mathrm{U}(\mathrm{S}) 1.5$ open reading frame in a cell type-specific manner. J. Virol. 2002, 76, 743-754. [CrossRef]

568. Leopardi, R.; Roizman, B. The herpes simplex virus major regulatory protein ICP4 blocks apoptosis induced by the virus or by hyperthermia. Proc. Natl. Acad. Sci. USA 1996, 93, 9583-9587. [CrossRef]

569. Benetti, L.; Roizman, B. Herpes simplex virus protein kinase US3 activates and functionally overlaps protein kinase A to block apoptosis. Proc. Natl. Acad. Sci. USA 2004, 101, 9411-9416. [CrossRef]

570. Walters, M.S.; Kinchington, P.R.; Banfield, B.W.; Silverstein, S. Hyperphosphorylation of histone deacetylase 2 by alphaherpesvirus US3 kinases. J. Virol. 2010, 84, 9666-9676. [CrossRef]

571. Chuluunbaatar, U.; Roller, R.; Feldman, M.E.; Brown, S.; Shokat, K.M.; Mohr, I. Constitutive mTORC1 activation by a herpesvirus Akt surrogate stimulates mRNA translation and viral replication. Genes Dev. 2010, 24, 2627-2639. [CrossRef]

572. Chuluunbaatar, U.; Mohr, I. A herpesvirus kinase that masquerades as Akt: You don't have to look like Akt, to act like it. Cell Cycle 2011, 10, 2064-2068. [CrossRef]

573. Sloan, D.D.; Zahariadis, G.; Posavad, C.M.; Pate, N.T.; Kussick, S.J.; Jerome, K.R. CTL Are inactivated by herpes simplex virus-infected cells expressing a viral protein kinase. J. Immunol. 2003, 171, 6733-6741. [CrossRef] [PubMed]

574. Sen, J.; Liu, X.; Roller, R.; Knipe, D.M. Herpes simplex virus US3 tegument protein inhibits Toll-like receptor 2 signaling at or before TRAF6 ubiquitination. Virology 2013, 439, 65-73. [CrossRef] [PubMed] 
575. Wang, S.; Wang, K.; Lin, R.; Zheng, C. Herpes simplex virus 1 serine/threonine kinase US3 hyperphosphorylates IRF3 and inhibits beta interferon production. J. Virol. 2013, 87, 12814-12827. [CrossRef] [PubMed]

576. Qin, C.; Zhang, R.; Lang, Y.; Shao, A.; Xu, A.; Feng, W.; Han, J.; Wang, M.; He, W.; Yu, C.; et al. Bclaf1 critically regulates the type I interferon response and is degraded by alphaherpesvirus US3. PLoS Pathog. 2019, 15, e1007559. [CrossRef]

577. Rubio, R.M.; Mohr, I. Inhibition of ULK1 and Beclin1 by an $\alpha$-herpesvirus Akt-like Ser/Thr kinase limits autophagy to stimulate virus replication. Proc. Natl. Acad. Sci. USA 2019, 116, 26941-26950. [CrossRef]

578. Kato, A.; Arii, J.; Shiratori, I.; Akashi, H.; Arase, H.; Kawaguchi, Y. Herpes simplex virus 1 protein kinase Us3 phosphorylates viral envelope glycoprotein B and Regulates its expression on the cell surface. J. Virol. 2009, 83, 250-261. [CrossRef]

579. Rao, P.; Pham, H.T.; Kulkarni, A.; Yang, Y.; Liu, X.; Knipe, D.M.; Cresswell, P.; Yuan, W. Herpes simplex virus 1 glycoprotein B and US3 collaborate to inhibit CD1d antigen presentation and NKT cell function. J. Virol. 2011, 85, 8093-8104. [CrossRef]

580. Cunningham, C.; Davison, A.J.; Dolan, A.; Frame, M.C.; McGeoch, D.J.; Meredith, D.M.; Moss, H.W.; Orr, A.C. The UL13 virion protein of herpes simplex virus type 1 is phosphorylated by a novel virus-induced protein kinase. J. Gen. Virol. 1992, 73, 303-311. [CrossRef]

581. Smith, R.F.; Smith, T.F. Identification of new protein kinase-related genes in three herpesviruses, herpes simplex virus, varicellazoster virus, and Epstein-Barr virus. J. Virol. 1989, 63, 450-455. [CrossRef]

582. Chee, M.S.; Lawrence, G.L.; Barrell, B.G. Alpha-, beta- and gammaherpesviruses encode a putative phosphotransferase. J. Gen. Virol. 1989, 70, 1151-1160. [CrossRef]

583. Overton, H.A.; McMillan, D.J.; Klavinskis, L.S.; Hope, L.; Ritchie, A.J.; Wong-kai-in, P. Herpes simplex virus type 1 gene UL13 encodes a phosphoprotein that is a component of the virion. Virology 1992, 190, 184-192. [CrossRef]

584. Lemaster, S.; Roizman, B. Herpes simplex virus phosphoproteins. II. Characterization of the virion protein kinase and of the polypeptides phosphorylated in the virion. J. Virol. 1980, 35, 798-811. [CrossRef] [PubMed]

585. Kenyon, T.K.; Lynch, J.; Hay, J.; Ruyechan, W.; Grose, C. Varicella-zoster virus ORF47 protein serine kinase: Characterization of a cloned, biologically active phosphotransferase and two viral substrates, ORF62 and ORF63. J. Virol. 2001, 75, 8854-8858 [CrossRef] [PubMed]

586. Chee, M.S.; Bankier, A.T.; Beck, S.; Bohni, R.; Brown, C.M.; Cerny, R.; Horsnell, T.; Hutchison, C.A., III; Kouzarides, T.; Martignetti, J.A. Analysis of the protein-coding content of the sequence of human cytomegalovirus strain AD169. Curr. Top. Microbiol. Immunol. 1990, 154, 125-169. [CrossRef]

587. Lawrence, G.L.; Chee, M.; Craxton, M.A.; Gompels, U.A.; Honess, R.W.; Barrell, B.G. Human herpesvirus 6 is closely related to human cytomegalovirus. J. Virol. 1990, 64, 287-299. [CrossRef]

588. Ng, T.I.; Talarico, C.; Burnette, T.C.; Biron, K.; Roizman, B. Partial substitution of the functions of the herpes simplex virus 1 $\mathrm{U}(\mathrm{L}) 13$ gene by the human cytomegalovirus U(L)97 gene. Virology 1996, 225, 347-358. [CrossRef]

589. Daikoku, T.; Shibata, S.; Goshima, F.; Oshima, S.; Tsurumi, T.; Yamada, H.; Yamashita, Y.; Nishiyama, Y. Purification and characterization of the protein kinase encoded by the UL13 gene of herpes simplex virus type 2. Virology 1997, $235,82-93$. [CrossRef]

590. Coulter, L.J.; Moss, H.W.; Lang, J.; McGeoch, D.J. A mutant of herpes simplex virus type 1 in which the UL13 protein kinase gene is disrupted. J. Gen. Virol. 1993, 74, 387-395. [CrossRef]

591. Overton, H.; McMillan, D.; Hope, L.; Wong-Kai-In, P. Production of host shutoff-defective mutants of herpes simplex virus type 1 by inactivation of the UL13 gene. Virology 1994, 202, 97-106. [CrossRef]

592. Tanaka, M.; Nishiyama, Y.; Sata, T.; Kawaguchi, Y. The role of protein kinase activity expressed by the UL13 gene of herpes simplex virus 1: The activity is not essential for optimal expression of UL41 and ICP0. Virology 2005, 341, 301-312. [CrossRef]

593. Shibaki, T.; Suzutani, T.; Yoshida, I.; Ogasawara, M.; Azuma, M. Participation of type I interferon in the decreased virulence of the UL13 gene-deleted mutant of herpes simplex virus type 1. J. Interf. Cytokine Res. 2001, 21, 279-285. [CrossRef] [PubMed]

594. Ogle, W.O.; Ng, T.I.; Carter, K.L.; Roizman, B. The UL13 protein kinase and the infected cell type are determinants of posttranslational modification of ICP0. Virology 1997, 235, 406-413. [CrossRef] [PubMed]

595. Asai, R.; Ohno, T.; Kato, A.; Kawaguchi, Y. Identification of proteins directly phosphorylated by UL13 protein kinase from herpes simplex virus 1. Microbes Infect. 2007, 9, 1434-1438. [CrossRef] [PubMed]

596. Zhu, Z.; Du, T.; Zhou, G.; Roizman, B. The stability of herpes simplex virus 1 ICP0 early after infection is defined by the RING finger and the UL13 protein kinase. J. Virol. 2014, 88, 5437-5443. [CrossRef] [PubMed]

597. Eaton, H.E.; Saffran, H.A.; Wu, F.W.; Quach, K.; Smiley, J.R. Herpes simplex virus protein kinases US3 and UL13 modulate VP11/12 phosphorylation, virion packaging, and phosphatidylinositol 3-kinase/Akt signaling activity. J. Virol. 2014, 88, 7379-7388. [CrossRef]

598. Geiss, B.J.; Cano, G.L.; Tavis, J.E.; Morrison, L.A. Herpes simplex virus 2 VP22 phosphorylation induced by cellular and viral kinases does not influence intracellular localization. Virology 2004, 330, 74-81. [CrossRef]

599. Ng, T.I.; Ogle, W.O.; Roizman, B. UL13 protein kinase of herpes simplex virus 1 complexes with glycoprotein E and mediates the phosphorylation of the viral Fc receptor: Glycoproteins E and I. Virology 1998, 241, 37-48. [CrossRef]

600. Attrill, H.L.; Cumming, S.A.; Clements, J.B.; Graham, S.V. The herpes simplex virus type 1 US11 protein binds the coterminal UL12, UL13, and UL14 RNAs and regulates UL13 expression in vivo. J. Virol. 2002, 76, 8090-8100. [CrossRef]

601. Gershburg, S.; Geltz, J.; Peterson, K.E.; Halford, W.P.; Gershburg, E. The UL13 and US3 protein kinases of herpes simplex virus 1 cooperate to promote the assembly and release of mature, infectious virions. PLoS ONE 2015, 10, e0131420. [CrossRef] 
602. Sato, Y.; Koshizuka, T.; Ishibashi, K.; Hashimoto, K.; Ishioka, K.; Ikuta, K.; Yokota, S.-I.; Fujii, N.; Suzutani, T. Involvement of herpes simplex virus type 1 UL13 protein kinase in induction of SOCS genes, the negative regulators of cytokine signaling. Microbiol. Immunol. 2017, 61, 159-167. [CrossRef]

603. Yokota, S.; Yokosawa, N.; Okabayashi, T.; Suzutani, T.; Miura, S.; Jimbow, K.; Fujii, N. Induction of suppressor of cytokine signaling-3 by herpes simplex virus type 1 contributes to inhibition of the interferon signaling pathway. J. Virol. 2004, 78, 6282-6286. [CrossRef] [PubMed]

604. Frey, K.G.; Ahmed, C.M.I.; Dabelic, R.; Jager, L.D.; Noon-Song, E.N.; Haider, S.M.; Johnson, H.M.; Bigley, N.J. HSV-1-induced SOCS-1 expression in keratinocytes: Use of a SOCS-1 antagonist to block a novel mechanism of viral immune evasion. J. Immunol. 2009, 183, 1253-1262. [CrossRef] [PubMed]

605. Reichard, A.C.; Cheemarla, N.R.; Bigley, N.J. SOCS1/3 expression levels in HSV-1-infected, cytokine-polarized and -unpolarized macrophages. J. Interf. Cytokine Res. 2015, 35, 32-41. [CrossRef] [PubMed]

606. Pennisi, R.; Musarra-Pizzo, M.; Lei, Z.; Zhou, G.G.; Sciortino, M.T. VHS, US3 and UL13 viral tegument proteins are required for herpes simplex virus-induced modification of protein kinase R. Sci. Rep. 2020, 10, 5580. [CrossRef]

607. Merrick, W.C. Mechanism and regulation of eukaryotic protein synthesis. Microbiol. Rev. 1992, 56, 291-315. [CrossRef]

608. Kawaguchi, Y.; Van Sant, C.; Roizman, B. Eukaryotic elongation factor 1delta is hyperphosphorylated by the protein kinase encoded by the U(L)13 gene of herpes simplex virus 1. J. Virol. 1998, 72, 1731-1736. [CrossRef]

609. Perkins, D.; Pereira, E.F.R.; Gober, M.; Yarowsky, P.J.; Aurelian, L. The herpes simplex virus type 2 R1 protein kinase (ICP10 PK) blocks apoptosis in hippocampal neurons, involving activation of the MEK/MAPK survival pathway. J. Virol. 2002, 76, 1435-1449. [CrossRef]

610. Kawaguchi, Y.; Kato, K.; Tanaka, M.; Kanamori, M.; Nishiyama, Y.; Yamanashi, Y. Conserved protein kinases encoded by herpesviruses and cellular protein kinase cdc2 target the same phosphorylation site in eukaryotic elongation factor 1delta. J. Virol. 2003, 77, 2359-2368. [CrossRef]

611. Baines, J.D.; Roizman, B. The open reading frames UL3, UL4, UL10, and UL16 are dispensable for the replication of herpes simplex virus 1 in cell culture. J. Virol. 1991, 65, 938-944. [CrossRef]

612. Jun, P.; Strelow, L.; Herman, R.; Marsden, H.; Eide, T.; Haarr, L.; Leib, D. The UL4 gene of herpes simplex virus type 1 is dispensable for latency, reactivation and pathogenesis in mice. J. Gen. Virol. 1998, 79, 1603-1611. [CrossRef]

613. Worrad, D.M.; Caradonna, S. The herpes simplex virus type 2 UL3 open reading frame encodes a nuclear localizing phosphoprotein. Virology 1993, 195, 364-376. [CrossRef] [PubMed]

614. Yoshida, S.; Lee, L.F.; Yanagida, N.; Nazerian, K. Identification and characterization of a Marek's disease virus gene homologous to glycoprotein L of herpes simplex virus. Virology 1994, 204, 414-419. [CrossRef] [PubMed]

615. Ghiasi, H.; Perng, G.C.; Cai, S.; Nesburn, A.B.; Wechsler, S.L. The UL3 open reading frame of herpes simplex virus type 1 codes for a phosphoprotein. Virus Res. 1996, 44, 137-142. [CrossRef]

616. McGeoch, D.J.; Cunningham, C.; McIntyre, G.; Dolan, A. Comparative sequence analysis of the long repeat regions and adjoining parts of the long unique regions in the genomes of herpes simplex viruses types 1 and 2. J. Gen. Virol. 1991, 72, 3057-3075. [CrossRef]

617. Davison, A.J. DNA sequence of the US component of the varicella-zoster virus genome. EMBO J. 1983, 2, 2203-2209. [CrossRef]

618. Telford, E.A.R.; Watson, M.S.; McBride, K.; Davison, A.J. The DNA sequence of equine herpesvirus-1. Virology 1992, $189,304-316$. [CrossRef]

619. Dean, H.J.; Cheung, A.K. Identification of the pseudorabies virus UL4 and UL5 (helicase) genes. Virology 1994, $202,962-967$. [CrossRef]

620. Vlcek, C.; Benes, V.; Lu, Z.; Kutish, G.F.; Paces, V.; Rock, D.; Letchworth, G.J.; Schwyzer, M. Nucleotide sequence analysis of a 30-kb region of the bovine herpesvirus 1 genome which exhibits a colinear gene arrangement with the UL21 to UL4 genes of herpes simplex virus. Virology 1995, 210, 100-108. [CrossRef]

621. Yamada, H.; Jiang, Y.-M.; Zhu, H.-Y.; Inagaki-Ohara, K.; Nishiyama, Y. Nucleolar localization of the UL3 protein of herpes simplex virus type 2. J. Gen. Virol. 1999, 80, 2157-2164. [CrossRef]

622. Zheng, C.; Lin, F.; Wang, S.; Xing, J. A novel virus-encoded nucleocytoplasmic shuttling protein: The UL3 protein of herpes simplex virus type 1. J. Virol. Methods 2011, 177, 206-210. [CrossRef] [PubMed]

623. Eide, T.; Marsden, H.S.; Leib, D.A.; Cunningham, C.; Davison, A.J.; Langeland, N.; Haarr, L. Identification of the UL4 protein of herpes simplex virus type 1. J. Gen. Virol. 1998, 79, 3033-3038. [CrossRef] [PubMed]

624. Markovitz, N.S.; Roizman, B. Small dense nuclear bodies are the site of localization of herpes simplex virus $1 \mathrm{U}(\mathrm{L}) 3$ and U(L)4 proteins and of ICP22 only when the latter protein is present. J. Virol. 2000, 74, 523-528. [CrossRef] [PubMed]

625. Ward, P.L.; Taddeo, B.; Markovitz, N.S.; Roizman, B. Identification of a novel expressed open reading frame situated between genes U(L)20 and U(L)21 of the herpes simplex virus 1 genome. Virology 2000, 266, 275-285. [CrossRef] [PubMed]

626. Loret, S.; Lippé, R. Biochemical analysis of infected cell polypeptide (ICP)0, ICP4, ul7 and ul23 incorporated into extracellular herpes simplex virus type 1 virions. J. Gen. Virol. 2012, 93, 624-634. [CrossRef]

627. Roller, R.J.; Fetters, R. The herpes simplex virus 1 UL51 protein interacts with the UL7 protein and plays a role in its recruitment into the virion. J. Virol. 2015, 89, 3112-3122. [CrossRef] 
628. Albecka, A.; Owen, D.J.; Ivanova, L.; Brun, J.; Liman, R.; Davies, L.; Ahmed, M.F.; Colaco, S.; Hollinshead, M.; Graham, S.C.; et al. Dual function of the pUL7-pUL51 tegument protein complex in herpes simplex virus 1 infection. J. Virol. 2017, 91, 1-19. [CrossRef]

629. Xu, X.; Fan, S.; Zhou, J.; Zhang, Y.; Che, Y.; Cai, H.; Wang, L.; Guo, L.; Liu, L.; Li, Q. The mutated tegument protein UL7 attenuates the virulence of herpes simplex virus 1 by reducing the modulation of $\alpha-4$ gene transcription. Virol. J. 2016, 13, 1-12. [CrossRef]

630. Nozawa, N.; Daikoku, T.; Yamauchi, Y.; Takakuwa, H.; Goshima, F.; Yoshikawa, T.; Nishiyama, Y. Identification and characterization of the UL7 gene product of herpes simplex virus type 2. Virus Genes 2002, 24, 257-266. [CrossRef]

631. Feutz, E.; McLeland-Wieser, H.; Ma, J.; Roller, R.J. Functional interactions between herpes simplex virus pUL51, pUL7 and gE reveal cell-specific mechanisms for epithelial cell-to-cell spread. Virology 2019, 537, 84-96. [CrossRef]

632. Tanaka, M.; Sata, T.; Kawaguchi, Y. The product of the Herpes simplex virus 1 UL7 gene interacts with a mitochondrial protein, adenine nucleotide translocator 2. Virol. J. 2008, 5, 1-13. [CrossRef]

633. Nozawa, N.; Daikoku, T.; Koshizuka, T.; Yamauchi, Y.; Yoshikawa, T.; Nishiyama, Y. Subcellular Localization of herpes simplex virus type 1 UL51 protein and role of palmitoylation in golgi apparatus targeting. J. Virol. 2003, 77, 3204-3216. [CrossRef] [PubMed]

634. Kato, A.; Oda, S.; Watanabe, M.; Oyama, M.; Kozuka-Hata, H.; Koyanagi, N.; Maruzuru, Y.; Arii, J.; Kawaguchi, Y. Roles of the phosphorylation of herpes simplex virus 1 UL51 at a specific site in viral replication and pathogenicity. J. Virol. 2018, 92, 1-21. [CrossRef] [PubMed]

635. Nozawa, N.; Kawaguchi, Y.; Tanaka, M.; Kato, A.; Kato, A.; Kimura, H.; Nishiyama, Y. Herpes simplex virus type 1 UL51 protein is involved in maturation and egress of virus particles. J. Virol. 2005, 79, 6947-6956. [CrossRef] [PubMed]

636. Roller, R.J.; Haugo, A.C.; Yang, K.; Baines, J.D. The herpes simplex virus 1 UL51 gene product has cell type-specific functions in cell-to-cell spread. J. Virol. 2014, 88, 4058-4068. [CrossRef] [PubMed]

637. Oda, S.; Arii, J.; Koyanagi, N.; Kato, A.; Kawaguchi, Y. The interaction between herpes simplex virus 1 tegument proteins UL51 and UL14 and its role in virion morphogenesis. J. Virol. 2016, 90, 8754-8767. [CrossRef] [PubMed]

638. Maclean, C.A.; Robertson, L.M.; Jamieson, F.E. Characterization of the UL10 gene product of herpes simplex virus type and investigation of its role in vivo. J. Gen. Virol. 1993, 74, 975-983. [CrossRef] [PubMed]

639. MacLean, C.A.; Efstathiou, S.; Elliott, M.L.; Jamieson, F.E.; McGeoch, D.J. Investigation of herpes simplex virus type 1 genes encoding multiply inserted membrane proteins. J. Gen. Virol. 1991, 72, 897-906. [CrossRef]

640. Baines, J.D.; Wills, E.; Jacob, R.J.; Pennington, J.; Roizman, B. Glycoprotein M of herpes simplex virus 1 is incorporated into virions during budding at the inner nuclear membrane. J. Virol. 2007, 81, 800-812. [CrossRef]

641. Zhang, J.; Nagel, C.-H.; Sodeik, B.; Lippé, R. Early, active, and specific localization of herpes simplex virus type $1 \mathrm{gM}$ to nuclear membranes. J. Virol. 2009, 83, 12984-12997. [CrossRef]

642. El Kasmi, I.; Lippé, R. Herpes simplex virus $1 \mathrm{gN}$ partners with gM To modulate the viral fusion machinery. J. Virol. 2015, 89, 2313-2323. [CrossRef]

643. Crump, C.M.; Bruun, B.; Bell, S.; Pomeranz, L.E.; Minson, T.; Browne, H.M. Alphaherpesvirus glycoprotein M causes the relocalization of plasma membrane proteins. J. Gen. Virol. 2004, 85, 3517-3527. [CrossRef] [PubMed]

644. Striebinger, H.; Funk, C.; Raschbichler, V.; Bailer, S.M. Subcellular trafficking and functional relationship of the HSV-1 glycoproteins $\mathrm{N}$ and M. Viruses 2016, 8, 83. [CrossRef] [PubMed]

645. Fuchs, W.; Klupp, B.G.; Granzow, H.; Osterrieder, N.; Mettenleiter, T.C. The interacting UL31 and UL34 gene products of pseudorabies virus are involved in egress from the host-cell nucleus and represent components of primary enveloped but not mature virions. J. Virol. 2002, 76, 364-378. [CrossRef] [PubMed]

646. Schnee, M.; Ruzsics, Z.; Bubeck, A.; Koszinowski, U.H. Common and specific properties of Herpesvirus UL34/UL31 protein family members revealed by protein complementation assay. J. Virol. 2006, 80, 11658-11666. [CrossRef]

647. Striebinger, H.; Zhang, J.; Ott, M.; Funk, C.; Radtke, K.; Duron, J.; Ruzsics, Z.; Haas, J.; Lippé, R.; Bailer, S.M. Subcellular trafficking and functional importance of herpes simplex virus type 1 glycoprotein M domains. J. Gen. Virol. 2015, 96, 3313-3325. [CrossRef]

648. Leege, T.; Fuchs, W.; Granzow, H.; Kopp, M.; Klupp, B.G.; Mettenleiter, T.C. Effects of simultaneous deletion of pUL11 and glycoprotein $\mathrm{M}$ on virion maturation of herpes simplex virus type 1. J. Virol. 2009, 83, 896-907. [CrossRef]

649. Ren, Y.; Bell, S.; Zenner, H.L.; Kathy Lau, S.Y.; Crump, C.M. Glycoprotein M is important for the efficient incorporation of glycoprotein H-L into herpes simplex virus type 1 particles. J. Gen. Virol. 2012, 93, 319-329. [CrossRef]

650. Koyano, S.; Mar, E.C.; Stamey, F.R.; Inoue, N. Glycoproteins M and N of human herpesvirus 8 form a complex and inhibit cell fusion. J. Gen. Virol. 2003, 84, 1485-1491. [CrossRef]

651. El Kasmi, I.; Khadivjam, B.; Lackman, M.; Duron, J.; Bonneil, E.; Thibault, P.; Lippé, R. Extended synaptotagmin 1 interacts with herpes simplex virus 1 glycoprotein $\mathrm{M}$ and Negatively modulates virus-induced membrane fusion. J. Virol. 2018, 92. [CrossRef]

652. Cheshenko, N.; del Rosario, B.; Woda, C.; Marcellino, D.; Satlin, L.M.; Herold, B.C. Herpes simplex virus triggers activation of calcium-signaling pathways. J. Cell Biol. 2003, 163, 283-293. [CrossRef]

653. Kalamvoki, M.; Roizman, B. Bcl-2 blocks accretion or depletion of stored calcium but has no effect on the redistribution of IP3 receptor I mediated by glycoprotein E of herpes simplex virus 1. J. Virol. 2007, 81, 6316-6325. [CrossRef]

654. Boruchowicz, H.; Hawkins, J.; Cruz-Palomar, K.; Lippé, R. The XPO6 exportin mediates herpes simplex virus 1 gM nuclear release late in infection. J. Virol. 2020, 94. [CrossRef] [PubMed] 
655. Perez-Caballero, D.; Zang, T.; Ebrahimi, A.; McNatt, M.W.; Gregory, D.A.; Johnson, M.C.; Bieniasz, P.D. Tetherin inhibits HIV-1 release by directly tethering virions to cells. Cell 2009, 139, 499-511. [CrossRef] [PubMed]

656. Blondeau, C.; Pelchen-Matthews, A.; Mlcochova, P.; Marsh, M.; Milne, R.S.B.; Towers, G.J. Tetherin restricts herpes simplex virus 1 and is antagonized by glycoprotein M. J. Virol. 2013, 87, 13124-13133. [CrossRef] [PubMed]

657. MacLean, C.A.; Clark, B.; McGeoch, D.J. Gene UL11 of herpes simplex virus type 1 encodes a virion protein which is myristylated. J. Gen. Virol. 1989, 70, 3147-3157. [CrossRef] [PubMed]

658. Loomis, J.S.; Bowzard, J.B.; Courtney, R.J.; Wills, J.W. Intracellular trafficking of the UL11 tegument protein of herpes simplex virus type 1. J. Virol. 2001, 75, 12209-12219. [CrossRef] [PubMed]

659. Loomis, J.S.; Courtney, R.J.; Wills, J.W. Packaging determinants in the UL11 tegument protein of herpes simplex virus type 1. J. Virol. 2006, 80, 10534-10541. [CrossRef]

660. MacLean, C.A.; Dolan, A.; Jamieson, F.E.; McGeoch, D.J. The myristylated virion proteins of herpes simplex virus type 1: Investigation of their role in the virus life cycle. J. Gen. Virol. 1992, 73, 539-547. [CrossRef]

661. Baird, N.L.; Starkey, J.L.; Hughes, D.J.; Wills, J.W. Myristylation and palmitylation of HSV-1 UL11 are not essential for its function. Virology 2010, 397, 80-88. [CrossRef]

662. Loomis, J.S.; Courtney, R.J.; Wills, J.W. Binding partners for the UL11 tegument protein of herpes simplex virus type 1. J. Virol. 2003, 77, 11417-11424. [CrossRef]

663. Yeh, P.-C.; Meckes, D.G.; Wills, J.W. Analysis of the interaction between the UL11 and UL16 tegument proteins of herpes simplex virus. J. Virol. 2008, 82, 10693-10700. [CrossRef] [PubMed]

664. Farnsworth, A.; Wisner, T.W.; Johnson, D.C. Cytoplasmic Residues of herpes simplex virus glycoprotein gE required for secondary envelopment and binding of tegument proteins VP22 and UL11 to gE and gD. J. Virol. 2007, 81, 319-331. [CrossRef] [PubMed]

665. Han, J.; Chadha, P.; Meckes, D.G.; Baird, N.L.; Wills, J.W. Interaction and Interdependent packaging of tegument protein UL11 and glycoprotein E of herpes simplex virus. J. Virol. 2011, 85, 9437-9446. [CrossRef] [PubMed]

666. Fulmer, P.A.; Melancon, J.M.; Baines, J.D.; Kousoulas, K.G. UL20 protein functions precede and are required for the UL11 functions of herpes simplex virus type 1 cytoplasmic virion envelopment. J. Virol. 2007, 81, 3097-3108. [CrossRef]

667. Wright, P.E.; Dyson, H.J. Intrinsically disordered proteins in cellular signalling and regulation. Nat. Rev. Mol. Cell Biol. 2015, 16, 18-29. [CrossRef]

668. Metrick, C.M.; Koenigsberg, A.L.; Heldwein, E.E. Conserved outer tegument component UL11 from herpes simplex virus 1 is an intrinsically disordered, RNA-binding protein. mBio 2020, 11, 1-22. [CrossRef]

669. Baird, N.L.; Yeh, P.C.; Courtney, R.J.; Wills, J.W. Sequences in the UL11 tegument protein of herpes simplex virus that control association with detergent-resistant membranes. Virology 2008, 374, 315-321. [CrossRef]

670. Chadha, P.; Sarfo, A.; Zhang, D.; Abraham, T.; Carmichael, J.; Han, J.; Wills, J.W. Domain interaction studies of herpes simplex virus 1 tegument protein UL16 reveal its interaction with mitochondria. J. Virol. 2017, 91. [CrossRef]

671. Nalwanga, D.; Rempel, S.; Roizman, B.; Baines, J.D. The U(L)16 gene product of herpes simplex virus 1 is a virion protein that colocalizes with intranuclear capsid proteins. Virology 1996, 226, 236-242. [CrossRef]

672. Carmichael, J.C.; Wills, J.W. Differential requirements for gE, gI, and UL16 among herpes simplex virus 1 syncytial variants suggest unique modes of dysregulating the mechanism of cell-to-cell spread. J. Virol. 2019, 93, 1-20. [CrossRef]

673. Harper, A.L.; Meckes, D.G.; Marsh, J.A.; Ward, M.D.; Yeh, P.-C.; Baird, N.L.; Wilson, C.B.; Semmes, O.J.; Wills, J.W. Interaction domains of the UL16 and UL21 tegument proteins of herpes simplex virus. J. Virol. 2010, 84, 2963-2971. [CrossRef] [PubMed]

674. Meckes, D.G.; Wills, J.W. Structural rearrangement within an enveloped virus upon binding to the host cell. J. Virol. 2008, 82, 10429-10435. [CrossRef] [PubMed]

675. Yeh, P.-C.; Han, J.; Chadha, P.; Meckes, D.G.; Ward, M.D.; Semmes, O.J.; Wills, J.W. Direct and specific binding of the UL16 tegument protein of herpes simplex virus to the cytoplasmic tail of glycoprotein E. J. Virol. 2011, 85, 9425-9436. [CrossRef] [PubMed]

676. Han, J.; Chadha, P.; Starkey, J.L.; Wills, J.W. Function of glycoprotein E of herpes simplex virus requires coordinated assembly of three tegument proteins on its cytoplasmic tail. Proc. Natl. Acad. Sci. USA 2012, 109, 19798-19803. [CrossRef]

677. Gao, J.; Yan, X.; Banfield, B.W. Comparative analysis of UL16 mutants derived from multiple strains of herpes simplex virus 2 (HSV-2) and HSV-1 reveals species-specific requirements for the UL16 protein. J. Virol. 2018, 92. [CrossRef]

678. Carmichael, J.C.; Starkey, J.; Zhang, D.; Sarfo, A.; Chadha, P.; Wills, J.W.; Han, J. Glycoprotein D of HSV-1 is dependent on tegument protein UL16 for packaging and contains a motif that is differentially required for syncytia formation. Virology 2019, 527, 64-76. [CrossRef]

679. Hutchinson, L.; Goldsmith, K.; Snoddy, D.; Ghosh, H.; Graham, F.L.; Johnson, D.C. Identification and characterization of a novel herpes simplex virus glycoprotein, gK, involved in cell fusion. J. Virol. 1992, 66, 5603-5609. [CrossRef]

680. Foster, T.P.; Alvarez, X.; Kousoulas, K.G. Plasma membrane topology of syncytial domains of herpes simplex virus type 1 glycoprotein K (gK): The UL20 protein enables cell surface localization of gK but not gK-mediated cell-to-cell fusion. J. Virol. 2003, 77, 499-510. [CrossRef]

681. Hutchinson, L.; Roop-Beauchamp, C.; Johnson, D.C. Herpes simplex virus glycoprotein K is known to influence fusion of infected cells, yet is not on the cell surface. J. Virol. 1995, 69, 4556-4563. [CrossRef] 
682. Jayachandra, S.; Baghian, A.; Kousoulas, K.G. Herpes simplex virus type 1 glycoprotein K is not essential for infectious virus production in actively replicating cells but is required for efficient envelopment and translocation of infectious virions from the cytoplasm to the extracellular space. J. Virol. 1997, 71, 5012-5024. [CrossRef]

683. Hutchinson, L.; Graham, F.L.; Cai, W.; Debroy, C.; Person, S.; Johnson, D.C. Herpes simplex virus (HSV) glycoproteins B and K inhibit cell fusion induced by HSV syncytial mutants. Virology 1993, 196, 514-531. [CrossRef] [PubMed]

684. Avitabile, E.; Lombardi, G.; Campadelli-Fiume, G. Herpes simplex virus glycoprotein K, but not its syncytial allele, inhibits cell-cell fusion mediated by the four fusogenic glycoproteins, gD, gB, gH, and gL. J. Virol. 2003, 77, 6836-6844. [CrossRef] [PubMed]

685. Foster, T.P.; Melancon, J.M.; Baines, J.D.; Kousoulas, K.G. The herpes simplex virus type 1 UL20 protein modulates membrane fusion events during cytoplasmic virion morphogenesis and virus-induced cell fusion. J. Virol. 2004, 78, 5347-5357. [CrossRef] [PubMed]

686. Foster, T.P.; Rybachuk, G.V.; Kousoulas, K.G. Glycoprotein K specified by herpes simplex virus type 1 is expressed on virions as a golgi complex-dependent glycosylated species and functions in virion entry. J. Virol. 2001, 75, 12431-12438. [CrossRef]

687. Foster, T.P.; Chouljenko, V.N.; Kousoulas, K.G. Functional and physical interactions of the herpes simplex virus type 1 UL20 membrane protein with glycoprotein K. J. Virol. 2008, 82, 6310-6323. [CrossRef]

688. Chouljenko, V.N.; Iyer, A.V.; Chowdhury, S.; Kim, J.; Kousoulas, K.G. The herpes simplex virus type 1 UL20 protein and the amino terminus of glycoprotein $\mathrm{K}$ (gK) physically interact with gB. J. Virol. 2010, 84, 8596-8606. [CrossRef]

689. Chouljenko, V.N.; Iyer, A.V.; Chowdhury, S.; Chouljenko, D.V.; Kousoulas, K.G. The amino terminus of herpes simplex virus type 1 glycoprotein K (gK) modulates gB-mediated virus-induced cell fusion and virion egress. J. Virol. 2009, 83, 12301-12313. [CrossRef]

690. Jambunathan, N.; Chowdhury, S.; Subramanian, R.; Chouljenko, V.N.; Walker, J.D.; Kousoulas, K.G. Site-specific proteolytic cleavage of the amino terminus of herpes simplex virus glycoprotein $\mathrm{K}$ on virion particles inhibits virus entry. J. Virol. 2011, 85, 12910-12918. [CrossRef]

691. Lau, S.Y.K.; Crump, C.M. HSV-1 gm and the gK/pUL20 complex are important for the localization of gD and gH/L to viral assembly sites. Viruses 2015, 7, 915-938. [CrossRef]

692. Musarrat, F.; Jambunathan, N.; Rider, P.J.F.; Chouljenko, V.N.; Kousoulas, K.G. The amino terminus of herpes simplex virus 1 glycoprotein $\mathrm{K}(\mathrm{gK})$ is required for gb binding to akt, release of intracellular calcium, and fusion of the viral envelope with plasma membranes. J. Virol. 2018, 92. [CrossRef]

693. Saied, A.A.; Chouljenko, V.N.; Subramanian, R.; Kousoulas, K.G. A replication competent HSV-1(McKrae) with a mutation in the amino-terminus of Glycoprotein $\mathrm{K}(\mathrm{gK})$ is unable to infect mouse trigeminal ganglia after cornea infection. Curr. Eye Res. 2014, 39, 596-603. [CrossRef] [PubMed]

694. Matundan, H.H.; Mott, K.R.; Akhtar, A.A.; Breunig, J.J.; Ghiasi, H. Mutations within the pathogenic region of herpes simplex virus $1 \mathrm{gK}$ signal sequences alter cell surface expression and neurovirulence. J. Virol. 2015, 89, 2530-2542. [CrossRef] [PubMed]

695. Takakuwa, H.; Goshima, F.; Koshizuka, T.; Murata, T.; Daikoku, T.; Nishiyama, Y. Herpes simplex virus encodes a virionassociated protein which promotes long cellular processes in over-expressing cells. Genes Cells 2001, 6, 955-966. [CrossRef] [PubMed]

696. Muto, Y.; Goshima, F.; Ushijima, Y.; Kimura, H.; Nishiyama, Y. Generation and characterization of UL21-null herpes simplex virus type 1. Front. Microbiol. 2012, 3, 1-6. [CrossRef]

697. Le Sage, V.; Jung, M.; Alter, J.D.; Wills, E.G.; Johnston, S.M.; Kawaguchi, Y.; Baines, J.D.; Banfield, B.W. The herpes simplex virus 2 UL21 protein is essential for virus propagation. J. Virol. 2013, 87, 5904-5915. [CrossRef]

698. Metrick, C.M.; Heldwein, E.E. Novel structure and unexpected RNA-binding ability of the C-terminal domain of herpes simplex virus 1 tegument protein UL21. J. Virol. 2016, 90, 7007-7008. [CrossRef]

699. Sarfo, A.; Starkey, J.; Mellinger, E.; Zhang, D.; Chadha, P.; Carmichael, J.; Wills, J.W. The UL21 Tegument protein of herpes simplex virus 1 is differentially required for the syncytial phenotype. J. Virol. 2017, 91, 1-18. [CrossRef]

700. Jacobson, J.G.; Chen, S.H.; Cook, W.J.; Kramer, M.F.; Coen, D.M. Importance of the herpes simplex virus UL24 gene for productive ganglionic infection in mice. Virology 1998, 242, 161-169. [CrossRef]

701. Abdeljelil, N.B.; Rochette, P.A.; Pearson, A. The UL24 protein of herpes simplex virus 1 affects the sub-cellular distribution of viral glycoproteins involved in fusion. Virology 2013, 444, 263-273. [CrossRef]

702. Pearson, A.; Coen, D.M. Identification, localization, and regulation of expression of the UL24 protein of herpes simplex virus type 1. J. Virol. 2002, 76, 10821-10828. [CrossRef]

703. Dridi, S.; Richerioux, N.; Suarez, C.E.G.; Vanharen, M.; Sanabria-Solano, C.; Pearson, A. A Mutation in the UL24 gene abolishes expression of the newly identified UL24.5 protein of herpes simplex virus 1 and leads to an increase in pathogenicity in mice. J. Virol. 2018, 92. [CrossRef] [PubMed]

704. Rochette, P.A.; Bourget, A.; Sanabria-Solano, C.; Lahmidi, S.; Lavalleé, G.O.; Pearson, A. Mutation of UL24 impedes the dissemination of acute herpes simplex virus 1 infection from the cornea to neurons of trigeminal ganglia. J. Gen. Virol. 2015, 96, 2794-2805. [CrossRef]

705. Blakeney, S.; Kowalski, J.; Tummolo, D.; DeStefano, J.; Cooper, D.; Guo, M.; Gangolli, S.; Long, D.; Zamb, T.; Natuk, R.J.; et al. Herpes simplex virus type 2 UL24 gene is a virulence determinant in murine and guinea pig disease models. J. Virol. 2005, 79, 10498-10506. [CrossRef] [PubMed] 
706. Kniżewski, Ł.; Kinch, L.; Grishin, N.V.; Rychlewski, L.; Ginalski, K. Human herpesvirus 1 UL24 gene encodes a potential PD-(D/E)XK endonuclease. J. Virol. 2006, 80, 2575-2577. [CrossRef] [PubMed]

707. Lymberopoulos, M.H.; Pearson, A. Involvement of UL24 in herpes-simplex-virus-1-induced dispersal of nucleolin. Virology 2007, 363, 397-409. [CrossRef] [PubMed]

708. Bertrand, L.; Leiva-Torres, G.A.; Hyjazie, H.; Pearson, A. Conserved residues in the UL24 protein of herpes simplex virus 1 are important for dispersal of the nucleolar protein nucleolin. J. Virol. 2010, 84, 109-118. [CrossRef] [PubMed]

709. Leiva-Torres, G.A.; Rochette, P.A.; Pearson, A. Differential importance of highly conserved residues in UL24 for herpes simplex virus 1 replication in vivo and reactivation. J. Gen. Virol. 2010, 91, 1109-1116. [CrossRef] [PubMed]

710. Callé, A.; Ugrinova, I.; Epstein, A.L.; Bouvet, P.; Diaz, J.-J.; Greco, A. Nucleolin is required for an efficient herpes simplex virus type 1 infection. J. Virol. 2008, 82, 4762-4773. [CrossRef]

711. Box, J.K.; Paquet, N.; Adams, M.N.; Boucher, D.; Bolderson, E.; O’Byrne, K.J.; Richard, D.J. Nucleophosmin: From structure and function to disease development. BMC Mol. Biol. 2016, 17, 1-12. [CrossRef]

712. Lymberopoulos, M.H.; Bourget, A.; Abdeljelil, N.B.; Pearson, A. Involvement of the UL24 protein in herpes simplex virus 1-induced dispersal of B23 and in nuclear egress. Virology 2011, 412, 341-348. [CrossRef]

713. Xu, H.; Su, C.; Pearson, A.; Mody, C.H.; Zheng, C. Herpes simplex virus 1 UL24 abrogates the DNA sensing signal pathway by inhibiting NF- $\mathrm{B}$ activation. J. Virol. 2017, 91, 1-10. [CrossRef] [PubMed]

714. Shiba, C.; Daikoku, T.; Goshima, F.; Takakuwa, H.; Yamauchi, Y.; Koiwai, O.; Nishiyama, Y. The UL34 gene product of herpes simplex virus type 2 is a tail-anchored type II membrane protein that is significant for virus envelopment. J. Gen. Virol. 2000, 81, 2397-2405. [CrossRef] [PubMed]

715. Ye, G.-J.; Roizman, B. The essential protein encoded by the UL31 gene of herpes simplex virus 1 depends for its stability on the presence of UL34 protein. Proc. Natl. Acad. Sci. USA 2000, 97, 11002-11007. [CrossRef] [PubMed]

716. Funk, C.; Ott, M.; Raschbichler, V.; Nagel, C.H.; Binz, A.; Sodeik, B.; Bauerfeind, R.; Bailer, S.M. The herpes simplex virus protein pUL31 escorts nucleocapsids to sites of nuclear egress, a process coordinated by Its N-terminal domain. PLoS Pathog. 2015, 11, e1004957. [CrossRef]

717. Chang, Y.E.; van Sant, C.; Krug, P.W.; Sears, A.E.; Roizman, B. The null mutant of the U(L)31 gene of herpes simplex virus 1: Construction and phenotype in infected cells. J. Virol. 1997, 71, 8307-8315. [CrossRef]

718. Roller, R.J.; Zhou, Y.; Schnetzer, R.; Ferguson, J.; DeSalvo, D. Herpes simplex virus type 1 UL34 gene product is required for viral envelopment. J. Virol. 2000, 74, 117-129. [CrossRef]

719. Monier, K.; Armas, J.C.G.; Etteldorf, S.; Ghazal, P.; Sullivan, K.F. Annexation of the interchromosomal space during viral infection. Nat. Cell Biol. 2000, 2, 661-665. [CrossRef]

720. Simpson-Holley, M.; Baines, J.; Roller, R.; Knipe, D.M. Herpes simplex virus 1 UL31 and UL34 gene products promote the late maturation of viral replication compartments to the nuclear periphery. J. Virol. 2004, 78, 5591-5600. [CrossRef]

721. Reynolds, A.E.; Liang, L.; Baines, J.D. Conformational changes in the nuclear lamina induced by herpes simplex virus type 1 require genes UL31 and UL34. J. Virol. 2004, 78, 5564-5575. [CrossRef]

722. Morrison, A.L.; DeLassus, G.S. Breach of the nuclear lamina during assembly of herpes simplex viruses. Nucleus 2011, 2, 271-276. [CrossRef]

723. Klupp, B.G.; Granzow, H.; Fuchs, W.; Keil, G.M.; Finke, S.; Mettenleiter, T.C. Vesicle formation from the nuclear membrane is induced by coexpression of two conserved herpesvirus proteins. Proc. Natl. Acad. Sci. USA 2007, 104, 7241-7246. [CrossRef] [PubMed]

724. Desai, P.J.; Pryce, E.N.; Henson, B.W.; Luitweiler, E.M.; Cothran, J. Reconstitution of the Kaposi's sarcoma-associated herpesvirus nuclear egress complex and formation of nuclear membrane vesicles by coexpression of ORF67 and ORF69 gene products. J. Virol. 2012, 86, 594-598. [CrossRef] [PubMed]

725. Luitweiler, E.M.; Henson, B.W.; Pryce, E.N.; Patel, V.; Coombs, G.; McCaffery, J.M.; Desai, P.J. Interactions of the Kaposi's sarcoma-associated herpesvirus nuclear egress complex: ORF69 is a potent factor for remodeling cellular membranes. J. Virol. 2013, 87, 3915-3929. [CrossRef] [PubMed]

726. Bigalke, J.M.; Heldwein, E.E. Structural basis of membrane budding by the nuclear egress complex of herpesviruses. EMBO J. 2015, 34, 2921-2936. [CrossRef] [PubMed]

727. Zeev-Ben-Mordehai, T.; Weberruß, M.; Lorenz, M.; Cheleski, J.; Hellberg, T.; Whittle, C.; el Omari, K.; Vasishtan, D.; Dent, K.C.; Harlos, K.; et al. Crystal structure of the herpesvirus nuclear egress complex provides insights into inner nuclear membrane remodeling. Cell Rep. 2015, 13, 2645-2652. [CrossRef]

728. Arii, J.; Watanabe, M.; Maeda, F.; Tokai-Nishizumi, N.; Chihara, T.; Miura, M.; Maruzuru, Y.; Koyanagi, N.; Kato, A.; Kawaguchi, Y. ESCRT-III mediates budding across the inner nuclear membrane and regulates its integrity. Nat. Commun. 2018, 9, 3379. [CrossRef]

729. Tandon, R.; Mocarski, E.S.; Conway, J.F. The A, B, Cs of herpesvirus capsids. Viruses 2015, 7, 899-914. [CrossRef]

730. Newcomb, W.W.; Homa, F.L.; Brown, J.C. Herpes simplex virus capsid structure: DNA packaging protein UL25 is located on the external surface of the capsid near the vertices. J. Virol. 2006, 80, 6286-6294. [CrossRef]

731. Yang, K.; Baines, J.D. Selection of HSV capsids for envelopment involves interaction between capsid surface components pU L31, pU L17, and pU L25. Proc. Natl. Acad. Sci. USA 2011, 108, 14276-14281. [CrossRef] 
732. Chen, D.-H.; Jakana, J.; McNab, D.; Mitchell, J.; Zhou, Z.H.; Dougherty, M.; Chiu, W.; Rixon, F.J. The Pattern of tegument-capsid interaction in the herpes simplex virus type 1 virion is not influenced by the small hexon-associated protein VP26. J. Virol. 2001, 75, 11863-11867. [CrossRef]

733. Zhou, Z.H.; He, J.; Jakana, J.; Tatman, J.D.; Rixon, F.J.; Chiu, W. Assembly of VP26 in herpes simplex virus-1 inferred from structures of wild-type and recombinant capsids. Nature 1995, 2, 1026-1030. [CrossRef] [PubMed]

734. Bowman, B.R.; Baker, M.L.; Rixon, F.J.; Chiu, W.; Quiocho, F.A. Structure of the herpesvirus major capsid protein. EMBO J. 2003, 22, 757-765. [CrossRef] [PubMed]

735. Lee, J.H.; Vittone, V.; Diefenbach, E.; Cunningham, A.L.; Diefenbach, R.J. Identification of structural protein-protein interactions of herpes simplex virus type 1. Virology 2008, 378, 347-354. [CrossRef] [PubMed]

736. Rixon, F.J.; Addison, C.; McGregor, A.; Macnab, S.J.; Nicholson, P.; Preston, V.G.; Tatman, J.D. Multiple interactions control the intracellular localization of the herpes simplex virus type 1 capsid proteins. J. Gen. Virol. 1996, 77, 2251-2260. [CrossRef]

737. Thomsen, D.R.; Roof, L.L.; Homa, F.L. Assembly of herpes simplex virus (HSV) intermediate capsids in insect cells infected with recombinant baculoviruses expressing HSV capsid proteins. J. Virol. 1994, 68, 2442-2457. [CrossRef]

738. Desai, P.; DeLuca, N.A.; Person, S. Herpes simplex virus type 1 VP26 is not essential for replication in cell culture but influences production of infectious virus in the nervous system of infected mice. Virology 1998, 247, 115-124. [CrossRef]

739. Kobayashi, R.; Kato, A.; Oda, S.; Koyanagi, N.; Oyama, M.; Kozuka-Hata, H.; Arii, J.; Kawaguchi, Y. Function of the herpes simplex virus 1 small capsid protein VP26 is regulated by phosphorylation at a specific site. J. Virol. 2015, 89, 6141-6147. [CrossRef]

740. Kobayashi, R.; Kato, A.; Sagara, H.; Watanabe, M.; Maruzuru, Y.; Koyanagi, N.; Arii, J.; Kawaguchi, Y. Herpes simplex virus 1 small capsomere-interacting protein VP26 regulates nucleocapsid maturation. J. Virol. 2017, 91. [CrossRef]

741. Douglas, M.W.; Diefenbach, R.J.; Homa, F.L.; Miranda-Saksena, M.; Rixon, F.J.; Vittone, V.; Byth, K.; Cunningham, A.L. Herpes simplex virus type 1 capsid protein VP26 interacts with dynein light chains RP3 and Tctex1 and plays a role in retrograde cellular transport. J. Biol. Chem. 2004, 279, 28522-28530. [CrossRef]

742. Apcarian, A.; Cunningham, A.L.; Diefenbach, R.J. Identification of binding domains in the herpes simplex virus type 1 small capsid protein pUL35 (VP26). J. Gen. Virol. 2010, 91, 2659-2663. [CrossRef]

743. Carter, K.L.; Ward, P.L.; Roizman, B. Characterization of the products of the U(L) 43 gene of herpes simplex virus 1: Potential implications for regulation of gene expression by antisense transcription. J. Virol. 1996, 70, 7663-7668. [CrossRef] [PubMed]

744. Loret, S.; Guay, G.; Lippé, R. Comprehensive characterization of extracellular herpes simplex virus type 1 virions. J. Virol. 2008, 82, 8605-8618. [CrossRef] [PubMed]

745. Herold, B.C.; WuDunn, D.; Soltys, N.; Spear, P.G. Glycoprotein C of herpes simplex virus type 1 plays a principal role in the adsorption of virus to cells and in infectivity. J. Virol. 1991, 65, 1090-1098. [CrossRef] [PubMed]

746. WuDunn, D.; Spear, P.G. Initial interaction of herpes simplex virus with cells is binding to heparan sulfate. J. Virol. 1989, 63, 52-58. [CrossRef]

747. Trybala, E.; Bergstrom, T.; Svennerholm, B.; Jeansson, S.; Glorioso, J.C.; Olofsson, S. Localization of a functional site on herpes simplex virus type 1 glycoprotein C involved in binding to cell surface heparan sulphate. J. Gen. Virol. 1994, 75, 743-752. [CrossRef]

748. Komala Sari, T.; Gianopulos, K.A.; Weed, D.J.; Schneider, S.M.; Pritchard, S.M.; Nicola, A.V. Herpes simplex virus glycoprotein c regulates low-pH Entry. mSphere 2020, 5, 1-16. [CrossRef]

749. Visalli, R.J.; Brandt, C.R. The HSV-1 UL45 18 kDa gene product is a true late protein and a component of the virion. Virus Res. 1993, 29, 167-178. [CrossRef]

750. Visalli, R.J.; Brandt, C.R. The HSV-1 UL45 gene product is not required for growth in vero cells. Virology 1991, 185, 419-423. [CrossRef]

751. Visalli, R.J.; Brandt, C.R. Mutation of the herpes simplex virus 1 KOS UL45 gene reveals dose dependent effects on central nervous system growth. Arch. Virol. 2002, 147, 519-532. [CrossRef]

752. Dollery, S.J.; Lane, K.D.; Delboy, M.G.; Roller, D.G.; Nicola, A.V. Role of the UL45 protein in herpes simplex virus entry via low $\mathrm{pH}$-dependent endocytosis and its relationship to the conformation and function of glycoprotein B. Virus Res. 2010, 149, 115-118. [CrossRef]

753. Haanes, E.J.; Nelson, C.M.; Soule, C.L.; Goodman, J.L. The UL45 gene product is required for herpes simplex virus type 1 glycoprotein B-induced fusion. J. Virol. 1994, 68, 5825-5834. [CrossRef] [PubMed]

754. Nicola, A.V.; Hou, J.; Major, E.O.; Straus, S.E. Herpes simplex virus type 1 enters human epidermal keratinocytes, but not neurons, via a pH-dependent endocytic pathway. J. Virol. 2005, 79, 7609-7616. [CrossRef] [PubMed]

755. Berkowitz, C.; Moyal, M.; Rossen-Wolff, A.; Darai, G.; Becker, Y. Herpes simplex virus type 1 (HSV-1) UL56 gene is involved in viral intraperitoneal pathogenicity to immunocompetent mice. Arch. Virol. 1994, 134, 73-83. [CrossRef] [PubMed]

756. Kolb, A.W.; Lee, K.; Larsen, I.; Craven, M.; Brandt, C.R. Quantitative trait locus based virulence determinant mapping of the HSV-1 genome in murine ocular infection: Genes involved in viral regulatory and innate immune networks contribute to virulence. PLoS Pathog. 2016, 12, e1005499. [CrossRef] [PubMed]

757. Nash, T.C.; Spivack, J.G. The UL55 and UL56 genes of herpes simplex virus type 1 are not required for viral replication, intraperitoneal virulence, or establishment of latency in mice. Virology 1994, 204, 794-798. [CrossRef] [PubMed] 
758. Umene, K.; Fukumaki, Y. DNA genome of spontaneously occurring deletion mutants of herpes simplex virus type 1 lacking one copy of the inverted repeat sequences of the L component. Arch. Virol. 2011, 156, 1305-1315. [CrossRef] [PubMed]

759. Kehm, R.; Gelderblom, H.R.; Darai, G. Identification of the UL56 protein of herpes simplex virus type 1 within the virion by immune electron microscopy. Virus Genes 1998, 17, 49-53. [CrossRef]

760. Koshizuka, T.; Kawaguchi, Y.; Goshima, F.; Mori, I.; Nishiyama, Y. Association of two membrane proteins encoded by herpes simplex virus type 2, UL11 and UL56. Virus Genes 2006, 32, 153-163. [CrossRef]

761. Ushijima, Y.; Koshizuka, T.; Goshima, F.; Kimura, H.; Nishiyama, Y. Herpes simplex virus type 2 UL56 interacts with the ubiquitin ligase Nedd4 and Increases its ubiquitination. J. Virol. 2008, 82, 5220-5233. [CrossRef]

762. Ushijima, Y.; Goshima, F.; Kimura, H.; Nishiyama, Y. Herpes simplex virus type 2 tegument protein UL56 relocalizes ubiquitin ligase Nedd4 and has a role in transport and/or release of virions. Virol. J. 2009, 6, 1-13. [CrossRef]

763. Ushijima, Y.; Luo, C.; Kamakura, M.; Goshima, F.; Kimura, H.; Nishiyama, Y. Herpes simplex virus UL56 interacts with and regulates the Nedd4-family ubiquitin ligase Itch. Virol. J. 2010, 7, 1-11. [CrossRef] [PubMed]

764. McGeoch, D.J. On the predictive recognition of signal peptide sequences. Virus Res. 1985, 3, 271-286. [CrossRef]

765. Weber, P.C.; Levine, M.; Glorioso, J.C. Rapid identification of nonessential genes of herpes simplex virus type 1 by Tn5 mutagenesis. Science 1987, 236, 576-579. [CrossRef] [PubMed]

766. Glück, B.; Möbius, S.; Pfaff, F.; Zell, R.; Sauerbrei, A. Novel method for genotyping clinical herpes simplex virus type 1 isolates. Arch. Virol. 2015, 160, 2807-2811. [CrossRef]

767. Jiang, Y.-M.; Yamada, H.; Goshima, F.; Daikoku, T.; Oshima, S.; Wada, K.; Nishiyama, Y. Characterization of the herpes simplex virus type 2 (HSV-2) US2 gene product and a US2-deficient HSV-2 mutant. J. Gen. Virol. 1998, 79, 1989-1995. [CrossRef]

768. Goshima, F.; Watanabe, D.; Suzuki, H.; Takakuwa, H.; Yamada, H.; Nishiyama, Y. The US2 gene product of herpes simplex virus type 2 interacts with cytokeratin 18. Arch. Virol. 2001, 146, 2201-2209. [CrossRef]

769. Norrild, B.; Lehto, V.P.; Virtanen, I. Organization of cytoskeleton elements during herpes simplex virus type I infection of human fibroblasts: An immunofluorescence study. J. Gen. Virol. 1986, 67, 97-105. [CrossRef]

770. Wu, Y.; Wei, F.; Tang, L.; Liao, Q.; Wang, H.; Shi, L.; Gong, Z.; Zhang, W.; Zhou, M.; Xiang, B.; et al. Herpesvirus acts with the cytoskeleton and promotes cancer progression. J. Cancer 2019, 10, 2185-2193. [CrossRef]

771. Mingo, R.M.; Han, J.; Newcomb, W.W.; Brown, J.C. Replication of herpes simplex virus: Egress of progeny virus at specialized cell membrane sites. J. Virol. 2012, 86, 7084-7097. [CrossRef]

772. Kang, M.-H.; Roy, B.B.; Finnen, R.L.; le Sage, V.; Johnston, S.M.; Zhang, H.; Banfield, B.W. The Us2 gene product of herpes simplex virus 2 is a membrane-associated ubiquitin-interacting protein. J. Virol. 2013, 87, 9590-9603. [CrossRef]

773. Lu, X.; Huang, C.; Zhang, Y.; Lin, Y.; Wang, X.; Li, Q.; Liu, S.; Tang, J.; Zhou, L. The Us2 gene product of herpes simplex virus 2 modulates NF-kB activation by targeting TAK1. Sci. Rep. 2017, 7, 1-11. [CrossRef] [PubMed]

774. Balan, P.; Davis-Poynter, N.; Bell, S.; Atkinson, H.; Browne, H.; Minson, T. An analysis of the in vitro and in vivo phenotypes of mutants of herpes simplex virus type 1 lacking glycoproteins gG, gE, gI or the putative gJ. J. Gen. Virol. 1994, 75, 1245-1258. [CrossRef] [PubMed]

775. Tran, L.C.; Kissner, J.M.; Westerman, L.E.; Sears, A.E. A herpes simplex virus 1 recombinant lacking the glycoprotein G coding sequences is defective in entry through apical surfaces of polarized epithelial cells in culture and in vivo. Proc. Natl. Acad. Sci. USA 2000, 97, 1818-1822. [CrossRef] [PubMed]

776. Ghiasi, H.; Kaiwar, R.; Nesburn, A.B.; Wechsler, S.L. Baculovirus-expressed glycoprotein g of herpes simplex virus type 1 partially protects vaccinated mice against lethal HSV-1 challenge. Virology 1992, 190, 233-239. [CrossRef]

777. Ghiasi, H.; Kaiwar, R.; Nesburn, A.B.; Slanina, S.; Wechsler, S.L. Expression of seven herpes simplex virus type 1 glycoproteins ( $\mathrm{gB}, \mathrm{gC}, \mathrm{gD}, \mathrm{gE}, \mathrm{gG}, \mathrm{gH}$, and gI): Comparative protection against lethal challenge in mice. J. Virol. 1994, 68, 2118-2126. [CrossRef]

778. Blacklaws, B.A.; Krishna, S.; Minson, A.C.; Nash, A.A. Immunogenicity of herpes simplex virus type 1 glycoproteins expressed in vaccinia virus recombinants. Virology 1990, 177, 727-736. [CrossRef]

779. Ghiasi, H.; Hofman, F.M.; Cai, S.; Perng, G.C.; Nesburn, A.B.; Wechsler, S.L. Vaccination with different HSV-1 glycoproteins induces different patterns of ocular cytokine responses following HSV-1 challenge of vaccinated mice. Vaccine 1999, 17, $2576-2582$. [CrossRef]

780. Baggiolini, M. Chemokines and leukocyte traffic. Nat. Immunol. 1998, 392, 949-952. [CrossRef]

781. Thapa, M.; Carr, D.J.J. CXCR3 deficiency increases susceptibility to genital herpes simplex virus type 2 infection: Uncoupling of CD8+ T-cell effector function but not migration. J. Virol. 2009, 83, 9486-9501. [CrossRef]

782. Richman, D.D.; Buckmaster, A.; Bell, S.; Hodgman, C.; Minson, A.C. Identification of a new glycoprotein of herpes simplex virus type 1 and genetic mapping of the gene that codes for it. J. Virol. 1986, 57, 647-655. [CrossRef]

783. Viejo-Borbolla, A.; Martinez-Martín, N.; Nel, H.J.; Rueda, P.; Martín, R.; Blanco, S.; Arenzana-Seisdedos, F.; Thelen, M.; Fallon, P.G.; Alcamí, A. Enhancement of chemokine function as an immunomodulatory strategy employed by human herpesviruses. PLoS Pathog. 2012, 8, e1002497. [CrossRef] [PubMed]

784. Martinez-Martin, N.; Viejo-Borbolla, A.; Martín, R.; Blanco, S.; Benovic, J.L.; Thelen, M.; Alcamí, A. Herpes simplex virus enhances chemokine function through modulation of receptor trafficking and oligomerization. Nat. Commun. 2015, 6. [CrossRef] [PubMed]

785. McLean, T.I.; Bachenheimer, S.L. Activation of cJUN N-terminal kinase by herpes simplex virus type 1 enhances viral replication. J. Virol. 1999, 73, 8415-8426. [CrossRef] [PubMed] 
786. Martínez-Martín, N.; Viejo-Borbolla, A.; Alcami, A. Herpes simplex virus particles interact with chemokines and enhance cell migration. J. Gen. Virol. 2016, 97, 3007-3016. [CrossRef]

787. Aubert, M.; Chen, Z.; Lang, R.; Dang, C.H.; Fowler, C.; Sloan, D.D.; Jerome, K.R. The antiapoptotic herpes simplex virus glycoprotein J localizes to multiple cellular organelles and induces reactive oxygen species formation. J. Virol. 2008, 82, 617-629. [CrossRef]

788. Jerome, K.R.; Chen, Z.; Lang, R.; Torres, M.R.; Hofmeister, J.; Smith, S.; Fox, R.; Froelich, C.J.; Corey, L. HSV and glycoprotein J inhibit caspase activation and apoptosis induced by granzyme B or fas. J. Immunol. 2001, 167, 3928-3935. [CrossRef]

789. Johnson, D.C.; Frame, M.C.; Ligas, M.W.; Cross, A.M.; Stow, N.D. Herpes simplex virus immunoglobulin G Fc receptor activity depends on a complex of two viral glycoproteins, gE and gI. J. Virol. 1988, 62, 1347-1354. [CrossRef]

790. Dingwell, K.S.; Brunetti, C.R.; Hendricks, R.L.; Tang, Q.; Tang, M.; Rainbow, A.J.; Johnson, D.C. Herpes simplex virus glycoproteins E and I facilitate cell-to-cell spread in vivo and across junctions of cultured cells. J. Virol. 1994, 68, 834-845. [CrossRef]

791. Dingwell, K.S.; Johnson, D.C. The herpes simplex virus gE-gI complex facilitates cell-to-cell spread and binds to components of cell junctions. J. Virol. 1998, 72, 8933-8942. [CrossRef]

792. Johnson, D.C.; Webb, M.; Wisner, T.W.; Brunetti, C. Herpes simplex virus gE/gI sorts nascent virions to epithelial cell junctions, promoting virus spread. J. Virol. 2001, 75, 821-833. [CrossRef]

793. Frank, I.; Friedman, H.M. A novel function of the herpes simplex virus type 1 Fc receptor: Participation in bipolar bridging of antiviral immunoglobulin G. J. Virol. 1989, 63, 4479-4488. [CrossRef] [PubMed]

794. Dubin, G.; Socolof, E.; Frank, I.; Friedman, H.M. Herpes simplex virus type 1 Fc receptor protects infected cells from antibodydependent cellular cytotoxicity. J. Virol. 1991, 65, 7046-7050. [CrossRef] [PubMed]

795. Van Vliet, K.E.; de Graaf-Miltenburg, L.A.; Verhoef, J.; Van Strijp, J.A. Direct evidence for antibody bipolar bridging on herpes simplex virus-infected cells. Immunology 1992, 77, 109-115. [PubMed]

796. Neidhardt, H.; Schröder, C.H.; Kaerner, H.C. Herpes simplex virus type 1 glycoprotein E is not indispensable for viral infectivity. J. Virol. 1987, 61, 600-603. [CrossRef]

797. Saldanha, C.E.; Lubinski, J.; Martin, C.; Nagashunmugam, T.; Wang, L.; van der Keyl, H.; Tal-Singer, R.; Friedman, H.M. Herpes simplex virus type 1 glycoprotein E domains involved in virus spread and disease. J. Virol. 2000, 74, 6712-6719. [CrossRef]

798. Polcicova, K.; Biswas, P.S.; Banerjee, K.; Wisner, T.W.; Rouse, B.T.; Johnson, D.C. Herpes keratitis in the absence of anterograde transport of virus from sensory ganglia to the cornea. Proc. Natl. Acad. Sci. USA 2005, 102, 11462-11467. [CrossRef]

799. Wang, F.; Tang, W.; McGraw, H.M.; Bennett, J.; Enquist, L.W.; Friedman, H.M. Herpes simplex virus type 1 glycoprotein E is required for axonal localization of capsid, tegument, and membrane glycoproteins. J. Virol. 2005, 79, 13362-13372. [CrossRef]

800. Snyder, A.; Polcicova, K.; Johnson, D.C. Herpes simplex virus gE/gI and US9 proteins promote transport of both capsids and virion glycoproteins in neuronal axons. J. Virol. 2008, 82, 10613-10624. [CrossRef]

801. Howard, P.W.; Wright, C.C.; Howard, T.; Johnson, D.C. Herpes simplex virus gE/gI extracellular domains promote axonal transport and spread from neurons to epithelial cells. J. Virol. 2014, 88, 11178-11186. [CrossRef]

802. LaVail, J.H.; Tauscher, A.N.; Sucher, A.; Harrabi, O.; Brandimarti, R. Viral regulation of the long distance axonal transport of herpes simplex virus nucleocapsid. Neuroscience 2007, 146, 974-985. [CrossRef]

803. Frame, M.C.; McGeoch, D.J.; Rixon, F.J.; Orr, A.C.; Marsden, H.S. The 10K virion phosphoprotein encoded by gene US9 from herpes simplex virus type 1. Virology 1986, 150, 321-332. [CrossRef]

804. Brideau, A.D.; Banfield, B.W.; Enquist, L.W. The Us9 gene product of pseudorabies virus, an alphaherpesvirus, is a phosphorylated, tail-anchored type II membrane protein. J. Virol. 1998, 72, 4560-4570. [CrossRef] [PubMed]

805. Brideau, A.D.; Eldridge, M.G.; Enquist, L.W. Directional transneuronal infection by pseudorabies virus is dependent on an acidic internalization motif in the Us9 cytoplasmic tail. J. Virol. 2000, 74, 4549-4561. [CrossRef] [PubMed]

806. DuRaine, G.; Wisner, T.W.; Howard, P.; Williams, M.; Johnson, D.C. Erratum for DuRaine et al., “Herpes simplex virus gE/gI and US9 promote both envelopment and sorting of virus particles in the cytoplasm of neurons, two processes that precede anterograde transport in axons". J. Virol. 2017, 91, 1-20. [CrossRef]

807. Zhang, W.; Gao, P.; Gui, X.; Zhou, L.; Ge, X.; Guo, X.; Wills, J.W.; Han, J.; Yang, H. Induction of rod-shaped structures by herpes simplex virus glycoprotein I. J. Virol. 2020, 94. [CrossRef]

808. Georgopoulou, U.; Michaelidou, A.; Roizman, B.; Mavromara-Nazos, P. Identification of a new transcriptional unit that yields a gene product within the unique sequences of the short component of the herpes simplex virus 1 genome. J. Virol. 1993, 67, 3961-3968. [CrossRef]

809. Willemse, M.J.; Strijdveen, I.G.L.; van Schooneveld, S.H.B.; van den Berg, M.C.; Sondermeijer, P.J.A. Transcriptional analysis of the short segment of the feline herpesvirus type 1 genome and insertional mutagenesis of a unique reading frame. Virology 1995, 208, 704-711. [CrossRef]

810. Yamada, H.; Daikoku, T.; Yamashita, Y.; Jiang, Y.M.; Tsurumi, T.; Nishiyama, Y. The product of the US10 gene of herpes simplex virus type 1 is a capsid/tegument-associated phosphoprotein which copurifies with the nuclear matrix. J. Gen. Virol. 1997, 78, 2923-2931. [CrossRef]

811. Johnson, P.A.; MacLean, C.; Marsden, H.S.; Dalziel, R.G.; Everett, R.D. The product of gene US11 of herpes simplex type 1 is expressed as a true late gene. J. Gen. Virol. 1986, 67, 871-883. [CrossRef]

812. Roller, R.J.; Roizman, B. The herpes simplex virus Us11 open reading frame encodes a sequence-specific RNA-binding protein. J. Virol. 1990, 64, 3463-3470. [CrossRef] 
813. Roller, R.J.; Roizman, B. Herpes simplex virus 1 RNA-binding protein US11 negatively regulates the accumulation of a truncated viral mRNA. J. Virol. 1991, 65, 5873-5879. [CrossRef] [PubMed]

814. Roller, R.J.; Roizman, B. A herpes simplex virus 1 US11-expressing cell line is resistant to herpes simplex virus infection at a step in viral entry mediated by glycoprotein D. J. Virol. 1994, 68, 2830-2839. [CrossRef] [PubMed]

815. Simonin, D.; Diaz, J.-J.; Kindbeiter, K.; Pernas, P.; Madjar, J.-J. Phosphorylation of herpes simplex virus type 1 Us11 protein is independent of viral genome expression. Electrophoresis 1995, 16, 1317-1322. [CrossRef] [PubMed]

816. Cassady, K.A.; Gross, M. The herpes simplex virus type 1 US11 protein interacts with protein kinase R in infected cells and requires a 30-amino-acid sequence adjacent to a kinase substrate domain. J. Virol. 2002, 76, 2029-2035. [CrossRef] [PubMed]

817. Greco, A.; Arata, L.; Soler, E.; Gaume, X.; Coute, Y.; Hacot, S.; Calle, A.; Monier, K.; Epstein, A.L.; Sanchez, J.-C.; et al. Nucleolin interacts with US11 protein of herpes simplex virus 1 and is involved in its trafficking. J. Virol. 2012, 86, 1449-1457. [CrossRef]

818. Diaz, J.-J.; Dodon, M.D.; Schaerer-Uthurralt, N.; Simonin, D.; Kindbeiter, K.; Gazzolo, L.; Madjar, J.-J. Post-transcriptional transctivation of human retroviral envelope glycoprotein expression by herpes simplex virus Us11 protein. Nature 1996, 379, 273-277. [CrossRef]

819. Schaerer-Uthurralt, N.; Erard, M.; Kindbeiter, K.; Madjar, J.J.; Diaz, J.J. Distinct domains in herpes simplex virus type 1 US11 protein mediate post-transcriptional transactivation of human T-lymphotropic virus type I envelope glycoprotein gene expression and specific binding to the Rex responsive element. J. Gen. Virol. 1998, 79, 1593-1602. [CrossRef]

820. Poppers, J.; Mulvey, M.; Khoo, D.; Mohr, I. Inhibition of PKR activation by the proline-rich RNA binding domain of the herpes simplex virus type 1 Us11 protein. J. Virol. 2000, 74, 11215-11221. [CrossRef]

821. Lussignol, M.; Queval, C.; Bernet-Camard, M.-F.; Cotte-Laffitte, J.; Beau, I.; Codogno, P.; Esclatine, A. The herpes simplex virus 1 Us11 protein inhibits autophagy through its interaction with the protein kinase PKR. J. Virol. 2013, 87, 859-871. [CrossRef]

822. Sadler, A.J.; Latchoumanin, O.; Hawkes, D.; Mak, J.; Williams, B.R.G. An antiviral response directed by PKR phosphorylation of the RNA helicase A. PLoS Pathog. 2009, 5, e1000311. [CrossRef]

823. Peters, G.A.; Khoo, D.; Mohr, I.; Sen, G.C. Inhibition of PACT-mediated activation of PKR by the herpes simplex virus type 1 Us11 protein. J. Virol. 2002, 76, 11054-11064. [CrossRef] [PubMed]

824. Kew, C.; Lui, P.-Y.; Chan, C.-P.; Liu, X.; Au, S.W.N.; Mohr, I.; Jin, D.-Y.; Kok, K.-H. Suppression of PACT-induced type I interferon production by herpes simplex virus 1 Us11 protein. J. Virol. 2013, 87, 13141-13149. [CrossRef] [PubMed]

825. Sànchez, R.; Mohr, I. Inhibition of cellular $2^{\prime}-5^{\prime}$ oligoadenylate synthetase by the herpes simplex virus type 1 Us11 protein. J. Virol. 2007, 81, 3455-3464. [CrossRef] [PubMed]

826. Liu, X.; Main, D.; Ma, Y.; He, B. Herpes simplex virus 1 inhibits TANK-binding kinase 1 through formation of the Us11-Hsp90 complex. J. Virol. 2018, 92. [CrossRef] [PubMed]

827. Liyana, A.; Vanessa, S.S. The emerging role of human TBK1 in virus-induced autophagy. Autophagy 2019, 15, 917-918. [CrossRef] [PubMed]

828. Diefenbach, R.J.; Miranda-Saksena, M.; Diefenbach, E.; Holland, D.J.; Boadle, R.A.; Armati, P.J.; Cunningham, A.L. Herpes simplex virus tegument protein US11 interacts with conventional kinesin heavy chain. J. Virol. 2002, 76, 3282-3291. [CrossRef]

829. Roller, R.J.; Monk, L.L.; Stuart, D.; Roizman, B. Structure and function in the herpes simplex virus 1 RNA-binding protein U(s)11: Mapping of the domain required for ribosomal and nucleolar association and RNA binding in vitro. J. Virol. 1996, 70, $2842-2851$. [CrossRef]

830. Benboudjema, L.; Mulvey, M.; Gao, Y.; Pimplikar, S.W.; Mohr, I. Association of the herpes simplex virus Type 1 Us11 gene product with the cellular kinesin light-chain-related protein PAT1 results in the redistribution of both polypeptides. J. Virol. 2003, 77, 9192-9203. [CrossRef]

831. Charron, A.J.; Ward, S.L.; North, B.J.; Ceron, S.; Leib, D.A. The US11 gene of herpes simplex virus 1 promotes neuroinvasion and periocular replication following corneal infection. J. Virol. 2019, 93, 1-19. [CrossRef]

832. Melancon, J.M.; Luna, R.E.; Foster, T.P.; Kousoulas, K.G. Herpes simplex virus type 1 gK is required for gB-mediated virus-induced cell fusion, while neither gB and gK nor gB and UL20p function redundantly in virion de-envelopment. J. Virol. 2005, 79, 299-313. [CrossRef]

833. Croft, C.L.; Noble, W. Preparation of organotypic brain slice cultures for the study of Alzheimer's disease [version 1; peer review: 3 approved]. F1000Research 2018, 7. [CrossRef] [PubMed]

834. Visalli, R.J.; Courtney, R.J.; Meyers, C. Infection and replication of herpes simplex virus type 1 in an organotypic epithelial culture system. Virology 1997, 230, 236-243. [CrossRef] [PubMed]

835. Webre, J.M.; Hill, J.M.; Nolan, N.M.; Clement, C.; McFerrin, H.E.; Bhattacharjee, P.S.; Hsia, V.; Neumann, D.M.; Foster, T.P.; Lukiw, W.J.; et al. Rabbit and mouse models of HSV-1 latency, reactivation, and recurrent eye diseases. J. Biomed. Biotechnol. $2012,2012$. [CrossRef] [PubMed]

836. Conlon, J.; Burdette, D.L.; Sharma, S.; Bhat, N.; Thompson, M.; Jiang, Z.; Rathinam, V.A.K.; Monks, B.; Jin, T.; Xiao, T.S.; et al. Mouse, but not human STING, binds and signals in response to the vascular disrupting agent 5,6-Dimethylxanthenone-4-Acetic acid. J. Immunol. 2013, 190, 5216-5225. [CrossRef] [PubMed]

837. Cavlar, T.; Deimling, T.; Ablasser, A.; Hopfner, K.P.; Hornung, V. Species-specific detection of the antiviral small-molecule compound CMA by STING. EMBO J. 2013, 32, 1440-1450. [CrossRef] [PubMed]

838. Sidhaye, J.; Knoblich, J.A. Brain organoids: An ensemble of bioassays to investigate human neurodevelopment and disease. Cell Death Differ. 2020. [CrossRef] 
839. Watanabe, M.; Buth, J.E.; Vishlaghi, N.; de la Torre-Ubieta, L.; Taxidis, J.; Khakh, B.S.; Coppola, G.; Pearson, C.A.; Yamauchi, K.; Gong, D.; et al. Self-organized cerebral organoids with human-specific features predict effective drugs to combat zika virus infection. Cell Rep. 2017, 21, 517-532. [CrossRef]

840. Qiao, H.; Guo, M.; Shang, J.; Zhao, W.; Wang, Z.; Liu, N.; Li, B.; Zhou, Y.; Wu, Y.; Chen, P. Herpes simplex virus type 1 infection leads to neurodevelopmental disorder-associated neuropathological changes. PLoS Pathog. 2020, 16, e1008899. [CrossRef]

841. D'Aiuto, L.; Bloom, D.C.; Naciri, J.N.; Smith, A.; Edwards, T.G.; Mcclain, L.; Callio, J.A.; Jessup, M.; Wood, J.; Chowdari, K.; et al. Modeling herpes simplex virus 1 infections in human central nervous system neuronal cells using two- and three dimensional cultures derived from induced pluripotent stem cells. J. Virol. 2019, 93. [CrossRef]

842. Edwards, T.G.; Bloom, D.C. Lund human mesencephalic (LUHMES) neuronal cell line supports herpes simplex virus 1 latency in vitro. J. Virol. 2019, 93,1-14. [CrossRef]

843. Dogrammatzis, C.; Waisner, H.; Kalamvoki, M. Cloaked viruses and viral factors in cutting edge exosome-based therapies. Front. Cell Dev. Biol. 2020, 8. [CrossRef] [PubMed]

844. Conry, R.M.; Westbrook, B.; McKee, S.; Norwood, T.G. Talimogene laherparepvec: First in class oncolytic virotherapy. Hum. Vaccines Immunother. 2018, 14, 839-846. [CrossRef] [PubMed]

845. Ghonime, M.G.; Jackson, J.; Shah, A.; Roth, J.; Li, M.; Saunders, U.; Coleman, J.; Gillespie, G.Y.; Markert, J.M.; Cassady, K.A. Chimeric HCMV /HSV-1 and $\Delta \gamma 134.5$ oncolytic herpes simplex virus elicit immune mediated antigliomal effect and antitumor memory. Transl. Oncol. 2018, 11, 86-93. [CrossRef] [PubMed]

846. Chiocca, E.A.; Nakashima, H.; Kasai, K.; Fernandez, S.A.; Oglesbee, M. Preclinical toxicology of rQNestin34.5v.2: An oncolytic herpes virus with transcriptional regulation of the ICP34.5 neurovirulence gene. Mol. Ther. Methods Clin. Dev. 2020, 17, 871-893. [CrossRef] [PubMed]

847. Sokolowski, N.A.; Rizos, H.; Diefenbach, R.J. Oncolytic virotherapy dovepress oncolytic virotherapy using herpes simplex virus: How far have we come? Oncolytic Virotherapy 2015, 4, 207-219.

848. Miao, L.; Fraefel, C.; Sia, K.C.; Newman, J.P.; Mohamed-Bashir, S.A.; Ng, W.H.; Lam, P.Y.P. The potential application of a transcriptionally regulated oncolytic herpes simplex virus for human cancer therapy. Br. J. Cancer 2014, 110, 94-106. [CrossRef]

849. Pyles, R.B.; Warnick, R.E.; Chalk, C.L.; Szanti, B.E.; Parysek, L.M. A novel multiply-mutated HSV-1 strain for the treatment of human brain tumors. Hum. Gene Ther. 1997, 8, 533-544. [CrossRef]

850. Stanfield, B.A.; Stahl, J.; Chouljenko, V.N.; Subramanian, R.; Charles, A.S.; Saied, A.A.; Walker, J.D.; Kousoulas, K.G. A single intramuscular vaccination of mice with the HSV-1 VC2 virus with mutations in the glycoprotein K and the membrane protein UL20 confers full protection against lethal intravaginal challenge with virulent HSV-1 and HSV-2 strains. PLoS ONE 2014, 9, e109890. [CrossRef]

851. Stanfield, B.A.; Pahar, B.; Chouljenko, V.N.; Veazey, R.; Kousoulas, K.G. Vaccination of rhesus macaques with the live-attenuated HSV-1 vaccine VC2 stimulates the proliferation of mucosal T cells and germinal center responses resulting in sustained production of highly neutralizing antibodies. Vaccine 2017, 35, 536-543. [CrossRef] 